\title{
Renormalized Coupled-Cluster Calculations of Reactive Potential Energy Surfaces: The BeFH System
}

\author{
Michael J. McGuire ${ }^{a}$ Piotr Piecuch,,${ }^{a, \dagger, \dagger, \dagger \dagger \dagger}$ Karol Kowalski, ${ }^{a}$ \\ Stanisław A. Kucharski, ${ }^{a, b}$ and Monika Musial ${ }^{b}$ \\ a Department of Chemistry, Michigan State University, East Lansing, Michigan 48824 \\ b Institute of Chemistry, University of Silesia, Szkolna 9, 40-006 Katowice, Poland
}

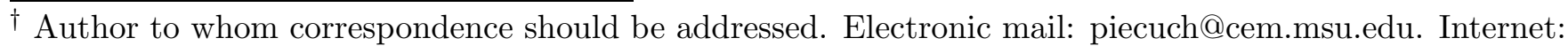
www.cem.msu.edu/ piecuch/group_web.

i† Alfred P. Sloan Research Fellow.

t† Also at: The Department of Physics and Astronomy, Michigan State University, East Lansing, Michigan 48824 .
} 
Table S1. The CCSD, CCSD (T), R-CCSD(T), CR-CCSD(T), and MRCI(Q) energies, in hartree, of the BeFH system, as described by the cc-pVTZ basis set, for the Be-F-H angle $\theta=180^{\circ}$. The $\mathrm{Be}-\mathrm{F}$ and $\mathrm{H}-\mathrm{F}$ bond distances, $R_{\mathrm{Be}-\mathrm{F}}$ and $R_{\mathrm{H}-\mathrm{F}}$, respectively, are in bohr.

\begin{tabular}{|c|c|c|c|c|c|c|}
\hline$R_{\mathrm{Be}-\mathrm{F}}$ & $R_{\mathrm{H}-\mathrm{F}}$ & CCSD & $\operatorname{CCSD}(\mathrm{T})$ & R-CCSD $(\mathrm{T})$ & CR-CCSD(T) & $\operatorname{MRCI}(\mathrm{Q})$ \\
\hline 1.8 & 1.2 & -114.358988 & -114.366391 & -114.365686 & -114.365288 & -114.364157 \\
\hline 1.8 & 1.4 & -114.503277 & -114.511160 & -114.510390 & -114.509952 & -114.508973 \\
\hline 1.8 & 1.6 & -114.555980 & -114.564461 & -114.563595 & -114.563114 & -114.562331 \\
\hline 1.8 & 1.7325 & -114.565835 & -114.574885 & -114.573898 & -114.573391 & -114.572792 \\
\hline 1.8 & 1.8 & -114.566637 & -114.576135 & -114.575024 & -114.574507 & -114.573980 \\
\hline 1.8 & 2.0 & -114.563369 & -114.578199 & -114.574809 & -114.574206 & -114.575231 \\
\hline 1.8 & 2.25 & -114.575724 & -114.615494 & -114.597407 & -114.593837 & -114.599264 \\
\hline 1.8 & 2.5 & -114.598294 & -114.633668 & -114.617840 & -114.615400 & -114.621754 \\
\hline 1.8 & 2.75 & -114.616827 & -114.647238 & -114.633609 & -114.632214 & -114.638546 \\
\hline 1.8 & 3.0 & -114.630648 & -114.659623 & -114.645869 & -114.644517 & -114.650517 \\
\hline 1.8 & 3.5 & -114.647839 & -114.677729 & -114.661762 & -114.659591 & -114.664766 \\
\hline 1.8 & 4.0 & -114.656333 & -114.687333 & -114.669607 & -114.666860 & -114.671472 \\
\hline 1.8 & 5.0 & -114.661916 & -114.692670 & -114.674122 & -114.671266 & -114.675509 \\
\hline 1.8 & 6.0 & -114.662877 & -114.692112 & -114.674273 & -114.671773 & -114.676004 \\
\hline 1.8 & 8.0 & -114.663058 & -114.689596 & -114.673561 & -114.671705 & -114.675984 \\
\hline 1.9 & 1.2 & -114.462764 & -114.470224 & -114.469490 & -114.469088 & -114.467879 \\
\hline 1.9 & 1.4 & -114.607237 & -114.615184 & -114.614385 & -114.613943 & -114.612898 \\
\hline 1.9 & 1.6 & -114.660068 & -114.668626 & -114.667730 & -114.667244 & -114.666415 \\
\hline 1.9 & 1.7325 & -114.670044 & -114.679184 & -114.678168 & -114.677654 & -114.677043 \\
\hline 1.9 & 1.8 & -114.670920 & -114.680508 & -114.679375 & -114.678849 & -114.678369 \\
\hline 1.9 & 2.0 & -114.667546 & -114.681596 & -114.678684 & -114.678086 & -114.678396 \\
\hline 1.9 & 2.25 & -114.677471 & -114.713303 & -114.698057 & -114.694918 & -114.700118 \\
\hline 1.9 & 2.5 & -114.698890 & -114.733528 & -114.718386 & -114.715789 & -114.721887 \\
\hline 1.9 & 2.75 & -114.716956 & -114.746684 & -114.733674 & -114.732198 & -114.738319 \\
\hline 1.9 & 3.0 & -114.730448 & -114.758139 & -114.745394 & -114.744176 & -114.750045 \\
\hline 1.9 & 3.5 & -114.747169 & -114.774734 & -114.760493 & -114.758726 & -114.763947 \\
\hline 1.9 & 4.0 & -114.755375 & -114.783660 & -114.767956 & -114.765667 & -114.770429 \\
\hline 1.9 & 5.0 & -114.760718 & -114.788973 & -114.772301 & -114.769818 & -114.774270 \\
\hline 1.9 & 6.0 & -114.761624 & -114.788821 & -114.772508 & -114.770284 & -114.774725 \\
\hline 1.9 & 8.0 & -114.761781 & -114.786928 & -114.771916 & -114.770225 & -114.774697 \\
\hline 2.0 & 1.2 & -114.539007 & -114.546515 & -114.545753 & -114.545349 & -114.544061 \\
\hline 2.0 & 1.4 & -114.683655 & -114.691656 & -114.690827 & -114.690382 & -114.689270 \\
\hline 2.0 & 1.6 & -114.736596 & -114.745217 & -114.744292 & -114.743802 & -114.742924 \\
\hline 2.0 & 1.7325 & -114.746641 & -114.755849 & -114.754808 & -114.754288 & -114.753650 \\
\hline 2.0 & 1.8 & -114.747542 & -114.757189 & -114.756041 & -114.755507 & -114.755040 \\
\hline 2.0 & 2.0 & -114.743807 & -114.757226 & -114.754680 & -114.754085 & -114.753814 \\
\hline 2.0 & 2.25 & -114.750992 & -114.783519 & -114.770594 & -114.767861 & -114.772811 \\
\hline 2.0 & 2.5 & -114.770951 & -114.805156 & -114.790515 & -114.787767 & -114.793667 \\
\hline 2.0 & 2.75 & -114.788439 & -114.817941 & -114.805273 & -114.803655 & -114.809625 \\
\hline 2.0 & 3.0 & -114.801559 & -114.828538 & -114.816432 & -114.815257 & -114.821038 \\
\hline 2.0 & 3.5 & -114.817790 & -114.843732 & -114.830717 & -114.829258 & -114.834530 \\
\hline 2.0 & 4.0 & -114.825719 & -114.851975 & -114.837779 & -114.835870 & -114.840771 \\
\hline 2.0 & 5.0 & -114.830848 & -114.857114 & -114.841927 & -114.839775 & -114.844422 \\
\hline 2.0 & 6.0 & -114.831709 & -114.857238 & -114.842174 & -114.840201 & -114.844844 \\
\hline 2.0 & 8.0 & -114.831847 & -114.855832 & -114.841680 & -114.840144 & -114.844808 \\
\hline 2.2 & 1.2 & -114.635469 & -114.643044 & -114.642230 & -114.641823 & -114.640902 \\
\hline 2.2 & 1.4 & -114.780371 & -114.788450 & -114.787567 & -114.787118 & -114.786203 \\
\hline
\end{tabular}


Table S1. (continued)

\begin{tabular}{|c|c|c|c|c|c|c|}
\hline$R_{\mathrm{Be}-\mathrm{F}}$ & $R_{\mathrm{H}-\mathrm{F}}$ & CCSD & $\operatorname{CCSD}(\mathrm{T})$ & $\mathrm{R}-\mathrm{CCSD}(\mathrm{T})$ & CR-CCSD $(\mathrm{T})$ & MRCI(Q) \\
\hline 2.2 & 1.6 & -114.833425 & -114.842134 & -114.841157 & -114.840660 & -114.839681 \\
\hline 2.2 & 1.7325 & -114.843473 & -114.852760 & -114.851679 & -114.851149 & -114.850433 \\
\hline 2.2 & 1.8 & -114.844322 & -114.854018 & -114.852849 & -114.852302 & -114.851787 \\
\hline 2.2 & 2.0 & -114.839488 & -114.851965 & -114.849916 & -114.849317 & -114.848575 \\
\hline 2.2 & 2.25 & -114.840715 & -114.867534 & -114.858401 & -114.856439 & -114.860752 \\
\hline 2.2 & 2.5 & -114.856756 & -114.890311 & -114.876559 & -114.873623 & -114.879214 \\
\hline 2.2 & 2.75 & -114.872756 & -114.902703 & -114.890198 & -114.888189 & -114.893956 \\
\hline 2.2 & 3.0 & -114.885031 & -114.911806 & -114.900234 & -114.898928 & -114.904591 \\
\hline 2.2 & 3.5 & -114.900274 & -114.924453 & -114.912891 & -114.911804 & -114.917150 \\
\hline 2.2 & 4.0 & -114.907702 & -114.931362 & -114.919142 & -114.917796 & -114.922911 \\
\hline 2.2 & 5.0 & -114.912490 & -114.935936 & -114.922869 & -114.921263 & -114.926235 \\
\hline 2.2 & 6.0 & -114.913290 & -114.936365 & -114.923155 & -114.921618 & -114.926607 \\
\hline 2.2 & 8.0 & -114.913395 & -114.935639 & -114.922807 & -114.921546 & -114.926554 \\
\hline 2.4 & 1.2 & -114.686313 & -114.693922 & -114.693062 & -114.692654 & -114.692402 \\
\hline 2.4 & 1.4 & -114.831322 & -114.839439 & -114.838511 & 8060 & 37775 \\
\hline 2.4 & 1.6 & -114.884345 & -114.893093 & -114.892073 & -114.891574 & -114.890666 \\
\hline 2.4 & 1.7325 & -114.894268 & -114.903573 & -114.902460 & -114.901925 & -114.900902 \\
\hline 2.4 & 1.8 & -114 & -114.904662 & 3477 & 2924 & -114 \\
\hline 2.4 & 2.0 & -114 & -114.900692 & -114 & -114 & -114 \\
\hline 2.4 & 2.25 & -114.8 & -114.906111 & -114.899886 & 98551 & -114.901568 \\
\hline 2.4 & 2.5 & -114.894799 & -114.927099 & -114.914608 & -114.911767 & -114.916998 \\
\hline 2.4 & 2.75 & -114 & -114 & 7045 & -114 & 30209 \\
\hline 2.4 & 3.0 & 9998 & -114.947687 & 36006 & 34369 & 39925 \\
\hline 2.4 & 3.5 & -114.934248 & -114.958107 & -114.947099 & -114.946108 & -114.951482 \\
\hline 2.4 & 4.0 & -114.941253 & -114.963756 & -114.952556 & -114.951542 & -114.956791 \\
\hline 2.4 & 5.0 & -114 & -114 & -114 & -114 & -114 \\
\hline 2.4 & 6.0 & -114.946567 & -114.968132 & -114.956119 & 54924 & -114.960177 \\
\hline 2.4 & 8.0 & -114.946643 & -114.967808 & -114.955853 & -114.954817 & -114.960100 \\
\hline 2.5 & 1.2 & -114.701690 & -114.709302 & -114.708424 & -114.708016 & -114.707703 \\
\hline 2.5 & 1.4 & -114 & -114 & -114 & -114 & 3033 \\
\hline 2.5 & 1.6 & -114.899661 & -114.908412 & -114.907373 & -114.906874 & -114.906686 \\
\hline 2.5 & 1.7325 & -114.909485 & -114.918781 & -114.917654 & -114.917119 & -114.916151 \\
\hline 2.5 & 1.8 & -114.910114 & -114.919768 & -114.918575 & -114.918021 & -114.916931 \\
\hline 2.5 & 2.0 & -114.903371 & -114.914916 & -114.913258 & 12647 & -114.912851 \\
\hline 2.5 & 2.25 & -114.895776 & -114.915751 & -114.910642 & -114.909532 & -114.911757 \\
\hline 2.5 & 2.5 & -114.903264 & -114.934477 & -114.922872 & -114.920206 & -114.925231 \\
\hline 2.5 & 2.75 & -114.915850 & -114.947315 & -114.934619 & -114.932042 & -114.937538 \\
\hline 2.5 & 3.0 & -114.926534 & -114.954950 & -114.943068 & -114.941228 & -114.946739 \\
\hline 2.5 & 3.5 & -114.940275 & -114.964394 & -114.953418 & -114.952389 & -114.957770 \\
\hline 2.5 & 4.0 & -114.947090 & -114.969440 & -114.958486 & -114.957558 & -114.962859 \\
\hline 2.5 & 5.0 & -114.951549 & -114.972904 & -114.961538 & -114.960488 & -114.965794 \\
\hline 2.5 & 6.0 & -114.952296 & -114.973399 & -114.961788 & -114.960729 & -114.966100 \\
\hline 2.5 & 8.0 & -114.952358 & -114.973199 & -114.961542 & -114.960600 & -114.966009 \\
\hline 2.5719 & 1.2 & -114.710010 & -114.717620 & -114.716729 & -114.716323 & -114.715969 \\
\hline 2.5719 & 1.4 & -114.855001 & -114.863122 & -114.862162 & -114.861712 & -114.861251 \\
\hline 2.5719 & 1.6 & -114.907900 & -114.916646 & -114.915596 & -114.915097 & -114.914804 \\
\hline 2.5719 & 1.7325 & -114.917640 & -114.926923 & -114.925787 & -114.925252 & -114.925217 \\
\hline 2.5719 & 1.8 & -114.918201 & -114.927830 & -114.926633 & -114.926079 & -114.925050 \\
\hline 2.5719 & 2.0 & -114.911004 & -114.922377 & -114.920774 & -114.920161 & -114.920469 \\
\hline
\end{tabular}


Table S1. (continued)

\begin{tabular}{|c|c|c|c|c|c|c|}
\hline$R_{\mathrm{Be}-\mathrm{F}}$ & $R_{\mathrm{H}-\mathrm{F}}$ & CCSD & $\operatorname{CCSD}(\mathrm{T})$ & $\mathrm{R}-\mathrm{CCSD}(\mathrm{T})$ & CR-CCSD $(\mathrm{T})$ & MRCI(Q) \\
\hline 2.5719 & 2.25 & -114.901487 & -114.920182 & -114.915740 & -114.914753 & -114.916354 \\
\hline 2.5719 & 2.5 & -114.906499 & -114.936710 & -114.925854 & -114.923360 & -114.928213 \\
\hline 2.5719 & 2.75 & -114.917963 & -114.949710 & -114.937030 & -114.934372 & -114.939804 \\
\hline 2.5719 & 3.0 & -114.928177 & -114.957180 & -114.945120 & -114.943131 & -114.948612 \\
\hline 2.5719 & 3.5 & -114.941537 & -114.965990 & -114.954954 & -114.953868 & -114.959252 \\
\hline 2.5719 & 4.0 & -114.948220 & -114.970613 & -114.959745 & -114.958849 & -114.964182 \\
\hline 2.5719 & 5.0 & -114.952618 & -114.973774 & -114.962619 & -114.961657 & -114.967028 \\
\hline 2.5719 & 6.0 & -114.953355 & -114.974233 & -114.962842 & -114.961867 & -114.967315 \\
\hline 2.5719 & 8.0 & -114.953408 & -114.974094 & -114.962602 & -114.961720 & -114.967214 \\
\hline 2.6 & 1.2 & -114.712766 & -114.720374 & -114.719479 & -114.719072 & -114.718704 \\
\hline 2.6 & 1.4 & -114.857745 & -114.865864 & -114.864899 & -114.864450 & -114.864128 \\
\hline 2.6 & 1.6 & -114.910616 & -114.919358 & -114.918304 & -114.917805 & -114.917476 \\
\hline 2.6 & 1.7325 & -114.920320 & -114.929596 & -114.928458 & -114.927923 & -114.927812 \\
\hline 2.6 & 1.8 & -114.920854 & -114.930471 & -114.929273 & -114.928719 & -114.928078 \\
\hline 2.6 & 2.0 & -114.913481 & -114.924790 & 23206 & 22593 & 22914 \\
\hline 2.6 & 2.25 & -114.903242 & -114.921478 & -114.917266 & -114.916319 & -114.917672 \\
\hline 2.6 & 2.5 & -114.907255 & -114.937026 & -114.926484 & -114.924067 & -114.928844 \\
\hline 2.6 & 2.75 & -114 & -114.950071 & -114 & 1733 & 0140 \\
\hline 2.6 & 3.0 & 8257 & -114.957498 & 45366 & 43318 & 8788 \\
\hline 2.6 & 3.5 & -114.941464 & -114.966078 & -114.955002 & -114.953887 & -114.959272 \\
\hline 2.6 & 4.0 & -114.948096 & -114.970538 & -114.959684 & -114.958794 & -114.964139 \\
\hline 2.6 & 5.0 & 52471 & -114.973576 & -11 & -114 & -114.966949 \\
\hline 2.6 & 6.0 & 53203 & -114.974016 & -114.962696 & 1751 & 967229 \\
\hline 2.6 & 8.0 & -114.953253 & -114.973897 & -114.962458 & -114.961597 & -114.967123 \\
\hline 2.7 & 1.2 & -114.720774 & -114.728368 & -114.727460 & -114.727055 & -114.726633 \\
\hline 2.7 & 1.4 & -114 & -114 & -114 & -114 & 371982 \\
\hline 2.7 & 1.6 & -114.918443 & -114.927167 & -114.926100 & 25602 & 925167 \\
\hline 2.7 & 1.7325 & -114.928012 & -114.937256 & -114.936108 & -114.935575 & -114.935286 \\
\hline 2.7 & 1.8 & -114.928443 & -114.938013 & -114.936811 & -114.936257 & -114.936112 \\
\hline 2.7 & 2.0 & 0458 & -114 & 0026 & -114 & -114.929713 \\
\hline 2.7 & 2.25 & -114.907794 & -114.924570 & -114.921059 & -114.920220 & -114.920717 \\
\hline 2.7 & 2.5 & -114.908151 & -114.936157 & -114.926814 & -114.924699 & -114.929163 \\
\hline 2.7 & 2.75 & -114.917233 & -114.949197 & -114.936722 & -114.934011 & -114.939320 \\
\hline 2.7 & 3.0 & -114.926474 & -114.956575 & -114.944181 & -114 & -114.947369 \\
\hline 2.7 & 3.5 & -114.939110 & -114.964422 & -114.953133 & -114.951893 & -114.957277 \\
\hline 2.7 & 4.0 & -114.945557 & -114.968313 & -114.957431 & -114.956535 & -114.961913 \\
\hline 2.7 & 5.0 & -114.949850 & -114.970896 & -114.959965 & -114.959117 & -114.964593 \\
\hline 2.7 & 6.0 & -114.950569 & -114.971248 & -114.960119 & -114.959265 & -114.964843 \\
\hline 2.7 & 8.0 & -114.950608 & -114.971172 & -114.959877 & -114.959083 & -114.964721 \\
\hline 2.9 & 1.2 & -114.731005 & -114.738546 & -114.737621 & -114.737220 & -114.736695 \\
\hline 2.9 & 1.4 & -114.875713 & -114.883762 & -114.882766 & -114.882321 & -114.881821 \\
\hline 2.9 & 1.6 & -114.928162 & -114.936818 & -114.935735 & -114.935241 & -114.934682 \\
\hline 2.9 & 1.7325 & -114.937426 & -114.946576 & -114.945418 & -114.944888 & -114.944431 \\
\hline 2.9 & 1.8 & -114.937644 & -114.947092 & -114.945886 & -114.945337 & -114.944953 \\
\hline 2.9 & 2.0 & -114.928523 & -114.939236 & -114.937790 & -114.937180 & -114.935989 \\
\hline 2.9 & 2.25 & -114.911736 & -114.926321 & -114.923739 & -114.923007 & -114.922560 \\
\hline 2.9 & 2.5 & -114.904713 & -114.928621 & -114.921778 & -114.920281 & -114.923821 \\
\hline 2.9 & 2.75 & -114.908960 & -114.940150 & -114.928645 & -114.926128 & -114.931187 \\
\hline 2.9 & 3.0 & -114.916176 & -114.947790 & -114.935022 & -114.932513 & -114.937849 \\
\hline
\end{tabular}


Table S1. (continued)

\begin{tabular}{|c|c|c|c|c|c|c|}
\hline$R_{\mathrm{Be}-\mathrm{F}}$ & $R_{\mathrm{H}-\mathrm{F}}$ & CCSD & $\operatorname{CCSD}(\mathrm{T})$ & R-CCSD $(\mathrm{T})$ & CR-CCSD(T) & $\operatorname{MRCI}(\mathrm{Q})$ \\
\hline 2.9 & 3.5 & -114.927442 & -114.954654 & -114.942665 & -114.941101 & -114.946471 \\
\hline 2.9 & 4.0 & -114.933492 & -114.957472 & -114.946194 & -114.945174 & -114.950597 \\
\hline 2.9 & 5.0 & -114.937615 & -114.959057 & -114.948130 & -114.947365 & -114.952975 \\
\hline 2.9 & 6.0 & -114.938305 & -114.959147 & -114.948133 & -114.947398 & -114.953152 \\
\hline 2.9 & 8.0 & -114.938327 & -114.959061 & -114.947851 & -114.947155 & -114.952997 \\
\hline 3.1 & 1.2 & -114.737180 & -114.744632 & -114.743704 & -114.743307 & -114.742686 \\
\hline 3.1 & 1.4 & -114.881598 & -114.889554 & -114.888555 & -114.888115 & -114.887523 \\
\hline 3.1 & 1.6 & -114.933699 & -114.942249 & -114.941163 & -114.940674 & -114.940874 \\
\hline 3.1 & 1.7325 & -114.942651 & -114.951670 & -114.950512 & -114.949989 & -114.949311 \\
\hline 3.1 & 1.8 & -114.942664 & -114.951958 & -114.950757 & -114.950215 & -114.949544 \\
\hline 3.1 & 2.0 & -114.932555 & -114.942926 & -114.941535 & -114.940933 & -114.941023 \\
\hline 3.1 & 2.25 & -114.912559 & -114.925677 & -114.923604 & -114.922910 & -114.923693 \\
\hline 3.1 & 2.5 & -114.898810 & -114.918741 & -114.914001 & -114.912947 & -114.915096 \\
\hline 3.1 & 2.75 & -114.896924 & -114.925672 & -114.916013 & -114.913982 & -114.918632 \\
\hline 3.1 & 3.0 & -114.901172 & -114.933439 & -114.920832 & -114.918315 & -114.923531 \\
\hline 3.1 & 3.5 & -114.910550 & -114.940105 & -114.927184 & -114.925269 & -114.930614 \\
\hline 3.1 & 4.0 & -114.916123 & -114.942025 & -114.929958 & -114.928704 & -114.934145 \\
\hline 3.1 & 5.0 & -114.920061 & -114. & -114.931229 & -114.930444 & -114 \\
\hline 3.1 & 6.0 & -114.920725 & -114.942247 & -114.931032 & -114.930342 & 36247 \\
\hline 3.1 & 8.0 & -114.920736 & -114.942035 & -114.930669 & -114.930025 & -114.936053 \\
\hline 3.3 & 1.2 & -114.741782 & -114.749114 & -114.748194 & -114.747803 & -114.747102 \\
\hline 3.3 & 1.4 & -114.885880 & -114 & -114.892719 & -114.892285 & 92365 \\
\hline 3.3 & 1.6 & -114.937618 & -114.946029 & -114.944950 & -114.944469 & -114.944624 \\
\hline 3.3 & 1.7325 & -114.946261 & -114.955118 & -114.953971 & -114.953456 & -114.953649 \\
\hline 3.3 & 1.8 & -114.946078 & -114.955192 & -114.954004 & -114.953471 & -114.953678 \\
\hline 3.3 & 2.0 & -114.935096 & -114.945151 & -114.943804 & -114.943213 & -114.942594 \\
\hline 3.3 & 2.25 & -114.912613 & -114.9 & -114.922929 & -114.922255 & -114.923609 \\
\hline 3.3 & 2.5 & -114.893355 & -114.910165 & -114.906824 & -114.905984 & -114.906812 \\
\hline 3.3 & 2.75 & -114.884697 & -114.909721 & -114.902397 & -114.900933 & -114.904828 \\
\hline 3.3 & 3.0 & -114.8 & -114.916151 & -114.904594 & -114.902375 & -114.907397 \\
\hline 3.3 & 3.5 & -114.891389 & -114.923355 & -114.909488 & -114.907304 & -114.912615 \\
\hline 3.3 & 4.0 & -114.896360 & -114.924741 & -114.911571 & -114.910032 & -114.915462 \\
\hline 3.3 & 5.0 & -114.900106 & -114.924167 & -114.912163 & -114.911276 & -114.917061 \\
\hline 3.3 & 6.0 & -114.900756 & -114.923429 & -114.911729 & -114.911020 & -114.917051 \\
\hline 3.3 & 8.0 & -114.900764 & -114.922986 & -114.911247 & -114.910619 & -114.916812 \\
\hline 3.5 & 1.2 & -114.745881 & -114.753067 & -114.752164 & -114.751779 & -114.751763 \\
\hline 3.5 & 1.4 & -114.889646 & -114.897325 & -114.896349 & -114.895922 & -114.895914 \\
\hline 3.5 & 1.6 & -114.941000 & -114.949245 & -114.948184 & -114.947710 & -114.947798 \\
\hline 3.5 & 1.7325 & -114.949329 & -114.958003 & -114.956874 & -114.956368 & -114.956508 \\
\hline 3.5 & 1.8 & -114.948956 & -114.957870 & -114.956703 & -114.956179 & -114.956338 \\
\hline 3.5 & 2.0 & -114.937190 & -114.946947 & -114.945638 & -114.945061 & -114.944595 \\
\hline 3.5 & 2.25 & -114.912771 & -114.924148 & -114.922518 & -114.921863 & -114.922881 \\
\hline 3.5 & 2.5 & -114.889339 & -114.903979 & -114.901441 & -114.900682 & -114.901740 \\
\hline 3.5 & 2.75 & -114.874306 & -114.895389 & -114.890189 & -114.889131 & -114.891793 \\
\hline 3.5 & 3.0 & -114.869070 & -114.897857 & -114.888249 & -114.886528 & -114.891166 \\
\hline 3.5 & 3.5 & -114.871670 & -114.905578 & -114.891084 & -114.888811 & -114.894076 \\
\hline 3.5 & 4.0 & -114.875821 & -114.907036 & -114.892564 & -114.890758 & -114.896155 \\
\hline 3.5 & 5.0 & -114.879368 & -114.905557 & -114.892527 & -114.891483 & -114.897302 \\
\hline 3.5 & 6.0 & -114.880024 & -114.904288 & -114.891835 & -114.891058 & -114.897186 \\
\hline
\end{tabular}


Table S1. (continued)

\begin{tabular}{|c|c|c|c|c|c|c|}
\hline$R_{\mathrm{Be}-\mathrm{F}}$ & $R_{\mathrm{H}-\mathrm{F}}$ & CCSD & $\operatorname{CCSD}(\mathrm{T})$ & R-CCSD $(\mathrm{T})$ & CR-CCSD(T) & MRCI(Q) \\
\hline 3.5 & 8.0 & -114.880041 & -114.903515 & -114.891205 & -114.890560 & -114.896898 \\
\hline 3.7 & 1.2 & -114.749770 & -114.756791 & -114.755910 & -114.755533 & -114.754719 \\
\hline 3.7 & 1.4 & -114.893195 & -114.900703 & -114.899749 & -114.899330 & -114.899225 \\
\hline 3.7 & 1.6 & -114.944160 & -114.952221 & -114.951182 & -114.950717 & -114.950717 \\
\hline 3.7 & 1.7325 & -114.952184 & -114.960657 & -114.959552 & -114.959057 & -114.959124 \\
\hline 3.7 & 1.8 & -114.951631 & -114.960331 & -114.959190 & -114.958678 & -114.958775 \\
\hline 3.7 & 2.0 & -114.939173 & -114.948646 & -114.947374 & -114.946811 & -114.946445 \\
\hline 3.7 & 2.25 & -114.913248 & -114.924068 & -114.922540 & -114.921906 & -114.922629 \\
\hline 3.7 & 2.5 & -114.886794 & -114.899984 & -114.897882 & -114.897161 & -114.899193 \\
\hline 3.7 & 2.75 & -114.866553 & -114.884392 & -114.880675 & -114.879809 & -114.881221 \\
\hline 3.7 & 3.0 & -114.855592 & -114.880655 & -114.873381 & -114.872127 & -114.875974 \\
\hline 3.7 & 3.5 & -114.852514 & -114.887253 & -114.872857 & -114.870730 & -114.875929 \\
\hline 3.7 & 4.0 & -114.855442 & -114.889538 & -114.873762 & -114.871781 & -114.877125 \\
\hline 3.7 & 5.0 & -114.858745 & -114.887556 & -114.873200 & -114.871973 & -114.877783 \\
\hline 3.7 & 6.0 & -114.859433 & -114.885722 & -114.872249 & -114.871368 & -114.877556 \\
\hline 3.7 & 8.0 & -114.859481 & -114.884527 & -114.871448 & -114.870763 & -114.877218 \\
\hline 3.9 & 1.2 & -114.753430 & -114.760279 & -114.759421 & -114.759052 & -114.757469 \\
\hline 3.9 & 1.4 & -114.896528 & -114.903856 & -114.902926 & -114.902516 & -114.900753 \\
\hline 3.9 & 1.6 & -114.947123 & -114.9 & -114.953978 & -114. & -114 \\
\hline 3.9 & 1.7325 & -114.954865 & -114.963132 & -114.962054 & -114.961569 & -114.961548 \\
\hline 3.9 & 1.8 & -114.954150 & -114.962635 & -114.961520 & -114.961020 & -114.961039 \\
\hline 3.9 & 2.0 & -114.941099 & -114.950305 & -114.949067 & -114.948519 & -114.948626 \\
\hline 3.9 & 2.25 & -114.914007 & -114.9 & -114.922928 & -114.922314 & -114.922083 \\
\hline 3.9 & 2.5 & -114.885409 & -114.897618 & -114.895756 & -114.895063 & -114.895171 \\
\hline 3.9 & 2.75 & -114.861327 & -114.876869 & -114.874023 & -114.873229 & -114.874879 \\
\hline 3.9 & 3.0 & -114.845134 & -114.866459 & -114.861203 & -114.860224 & -114.862785 \\
\hline 3.9 & 3.5 & -114.834800 & -114.8 & $-114 . \varepsilon$ & -114 & -114.858749 \\
\hline 3.9 & 4.0 & -114.835820 & -114.872393 & -114.855624 & -114.853622 & -114.858898 \\
\hline 3.9 & 5.0 & -114.838738 & -114.870606 & -114.854659 & -114.853261 & -114.859018 \\
\hline 3.9 & 6.0 & -114.839485 & -114.868239 & -114.853471 & -114.852470 & -114.858675 \\
\hline 3.9 & 8.0 & -114.839589 & -114.866543 & -114.852487 & -114.8 & -114.858288 \\
\hline 4.1 & 1.2 & -114.756769 & -114.763447 & -114.762613 & -114.762252 & -114.760637 \\
\hline 4.1 & 1.4 & -114.899569 & -114.906718 & -114.905812 & -114.905411 & -114.903617 \\
\hline 4.1 & 1.6 & -114.949830 & -114.957510 & -114.956521 & -114.956077 & -114.955329 \\
\hline 4.1 & 1.7325 & -114.957322 & -114.965389 & -114.964336 & -114.963863 & -114.963191 \\
\hline 4.1 & 1.8 & -114.956466 & -114.964742 & -114.963655 & -114.963166 & -114.962538 \\
\hline 4.1 & 2.0 & -114.942918 & -114.951877 & -114.950672 & -114.950138 & -114.949657 \\
\hline 4.1 & 2.25 & -114.914927 & -114.924941 & -114.923539 & -114.922944 & -114.922665 \\
\hline 4.1 & 2.5 & -114.884815 & -114.896338 & -114.894616 & -114.893949 & -114.893888 \\
\hline 4.1 & 2.75 & -114.858069 & -114.872085 & -114.869712 & -114.868955 & -114.871510 \\
\hline 4.1 & 3.0 & -114.837748 & -114.856098 & -114.852201 & -114.851329 & -114.852784 \\
\hline 4.1 & 3.5 & -114.819320 & -114.850803 & -114.839602 & -114.838211 & -114.842926 \\
\hline 4.1 & 4.0 & -114.817408 & -114.855503 & -114.838450 & -114.836600 & -114.841789 \\
\hline 4.1 & 5.0 & -114.819626 & -114.854879 & -114.837161 & -114.835639 & -114.841298 \\
\hline 4.1 & 6.0 & -114.820446 & -114.852120 & -114.835778 & -114.834657 & -114.840834 \\
\hline 4.1 & 8.0 & -114.820639 & -114.849871 & -114.834607 & -114.833796 & -114.840400 \\
\hline 4.5 & 1.2 & -114.762206 & -114.768579 & -114.767785 & -114.767440 & -114.765698 \\
\hline 4.5 & 1.4 & -114.904525 & -114.911355 & -114.910491 & -114.910107 & -114.908190 \\
\hline 4.5 & 1.6 & -114.954254 & -114.961595 & -114.960650 & -114.960225 & -114.959380 \\
\hline
\end{tabular}


Table S1. (continued)

\begin{tabular}{|c|c|c|c|c|c|c|}
\hline$R_{\mathrm{Be}-\mathrm{F}}$ & $R_{\mathrm{H}-\mathrm{F}}$ & CCSD & $\operatorname{CCSD}(\mathrm{T})$ & $\mathrm{R}-\mathrm{CCSD}(\mathrm{T})$ & CR-CCSD $(\mathrm{T})$ & $\operatorname{MRCI}(\mathrm{Q})$ \\
\hline 4.5 & 1.7325 & -114.961351 & -114.969062 & -114.968055 & -114.967602 & -114.966831 \\
\hline 4.5 & 1.8 & -114.960278 & -114.968186 & -114.967146 & -114.966679 & -114.965952 \\
\hline 4.5 & 2.0 & -114.945991 & -114.954533 & -114.953381 & -114.952872 & -114.952301 \\
\hline 4.5 & 2.25 & -114.916781 & -114.926248 & -114.924921 & -114.924358 & -114.924010 \\
\hline 4.5 & 2.5 & -114.884840 & -114.895492 & -114.893916 & -114.893292 & -114.893165 \\
\hline 4.5 & 2.75 & -114.855103 & -114.867435 & -114.865462 & -114.864758 & -114.864818 \\
\hline 4.5 & 3.0 & -114.829911 & -114.844838 & -114.842143 & -114.841331 & -114.841604 \\
\hline 4.5 & 3.5 & -114.797368 & -114.822085 & -114.815356 & -114.814300 & -114.816761 \\
\hline 4.5 & 4.0 & -114.786108 & -114.822825 & -114.808340 & -114.806995 & -114.811633 \\
\hline 4.5 & 5.0 & -114.784715 & -114.826903 & -114.805849 & -114.804298 & -114.809565 \\
\hline 4.5 & 6.0 & -114.785550 & -114.824469 & -114.804208 & -114.802916 & -114.808835 \\
\hline 4.5 & 8.0 & -114.786001 & -114.821185 & -114.802675 & -114.801724 & -114.808312 \\
\hline 4.7 & 1.2 & -114.764268 & -114.770512 & -114.769735 & -114.769396 & -114.767651 \\
\hline 4.7 & 1.4 & -114.906405 & -114.913101 & -114.912254 & -114.911877 & -114.909962 \\
\hline 4.7 & 1.6 & -114.955934 & -114.963133 & -114.962206 & -114.961789 & -114.960913 \\
\hline 4.7 & 1.7325 & -114.962884 & -114.970445 & -114.969458 & -114.969014 & -114.968211 \\
\hline 4.7 & 1.8 & -114.961730 & -114.969485 & -114.968464 & -114.968007 & -114.967249 \\
\hline 4.7 & 2.0 & -114.947182 & -114.955554 & -114.954423 & -114.953925 & -114.953324 \\
\hline 4.7 & 2.25 & -114.917570 & -114.926831 & -114.925530 & -114.924980 & -114.924609 \\
\hline 4.7 & 2.5 & -114.885086 & -114.895452 & -114.893918 & -114.893310 & -114 \\
\hline 4.7 & 2.75 & -114.854549 & -114.866410 & -114.864524 & -114.863840 & -114.863919 \\
\hline 4.7 & 3.0 & -114.828104 & -114.842151 & -114.839680 & -114.838888 & -114.839135 \\
\hline 4.7 & 3.5 & -114.790987 & -114 & -114.807705 & -114.806620 & -114.807335 \\
\hline 4.7 & 4.0 & -114.774323 & -114.808555 & -114.796398 & -114.795108 & -114.798880 \\
\hline 4.7 & 5.0 & -114.769231 & -114.814415 & -114.792357 & -114.790846 & -114.795700 \\
\hline 4.7 & 6.0 & -114.769791 & -114.813063 & -114.790569 & -114.789214 & -114.794814 \\
\hline 4.7 & 8.0 & -114.770355 & -114.809489 & -114.788827 & -114.7 & -114.794253 \\
\hline 5.0 & 1.2 & -114.766619 & -114.772702 & -114.771946 & -114.771615 & -114.769869 \\
\hline 5.0 & 1.4 & -114.908548 & -114.915075 & -114.914249 & -114.913882 & -114.911966 \\
\hline 5.0 & 1.6 & -114.957846 & -114.964867 & -114.963962 & -114.963556 & -114.962649 \\
\hline 5.0 & 1.7325 & -114.964628 & -114.972003 & -114.971039 & -114.970607 & -114.969773 \\
\hline 5.0 & 1.8 & -114.963383 & -114.970948 & -114.969951 & -114.969505 & -114.968716 \\
\hline 5.0 & 2.0 & -114.948543 & -114.956706 & -114.955602 & -114.955118 & -114.954486 \\
\hline 5.0 & 2.25 & -114.918505 & -114.927519 & -114.926250 & -114.925716 & -114.925321 \\
\hline 5.0 & 2.5 & -114.885480 & -114.895528 & -114.894037 & -114.893448 & -114.893310 \\
\hline 5.0 & 2.75 & -114.854201 & -114.865591 & -114.863781 & -114.863119 & -114.863245 \\
\hline 5.0 & 3.0 & -114.826672 & -114.839920 & -114.837615 & -114.836847 & -114.837238 \\
\hline 5.0 & 3.5 & -114.785561 & -114.805251 & -114.800827 & -114.799693 & -114.800447 \\
\hline 5.0 & 4.0 & -114.762508 & -114.793081 & -114.783700 & -114.782133 & -114.783147 \\
\hline 5.0 & 5.0 & -114.749421 & -114.798026 & -114.775833 & -114.774082 & -114.777589 \\
\hline 5.0 & 6.0 & -114.748548 & -114.799514 & -114.773533 & -114.771912 & -114.776412 \\
\hline 5.0 & 8.0 & -114.748979 & -114.796139 & -114.771245 & -114.769975 & -114.775808 \\
\hline 5.2 & 1.2 & -114.767769 & -114.773765 & -114.773019 & -114.772693 & -114.770947 \\
\hline 5.2 & 1.4 & -114.909594 & -114.916029 & -114.915215 & -114.914853 & -114.912936 \\
\hline 5.2 & 1.6 & -114.958777 & -114.965701 & -114.964808 & -114.964407 & -114.963489 \\
\hline 5.2 & 1.7325 & -114.965475 & -114.972749 & -114.971797 & -114.971371 & -114.970525 \\
\hline 5.2 & 1.8 & -114.964185 & -114.971646 & -114.970662 & -114.970223 & -114.969421 \\
\hline 5.2 & 2.0 & -114.949204 & -114.957255 & -114.956165 & -114.955688 & -114.955043 \\
\hline 5.2 & 2.25 & -114.918966 & -114.927851 & -114.926599 & -114.926073 & -114.925667 \\
\hline
\end{tabular}


Table S1. (continued)

\begin{tabular}{|c|c|c|c|c|c|c|}
\hline$R_{\mathrm{Be}-\mathrm{F}}$ & $R_{\mathrm{H}-\mathrm{F}}$ & CCSD & $\operatorname{CCSD}(\mathrm{T})$ & $\mathrm{R}-\mathrm{CCSD}(\mathrm{T})$ & CR-CCSD(T) & MRCI(Q) \\
\hline 5.2 & 2.5 & -114.885702 & -114.895591 & -114.894122 & -114.893542 & -114.893403 \\
\hline 5.2 & 2.75 & -114.854120 & -114.865292 & -114.863514 & -114.862863 & -114.863013 \\
\hline 5.2 & 3.0 & -114.826179 & -114.839094 & -114.836846 & -114.836091 & -114.836566 \\
\hline 5.2 & 3.5 & -114.783671 & -114.802452 & -114.798291 & -114.797144 & -114.798342 \\
\hline 5.2 & 4.0 & -114.758094 & -114.787057 & -114.778572 & -114.776785 & -114.778082 \\
\hline 5.2 & 5.0 & -114.739496 & -114.790138 & -114.768198 & -114.765763 & -114.766082 \\
\hline 5.2 & 6.0 & -114.736554 & -114.794026 & -114.765433 & -114.763187 & -114.764093 \\
\hline 5.2 & 8.0 & -114.736307 & -114.791357 & -114.762462 & -114.760728 & -114.765474 \\
\hline 5.5 & 1.2 & -114.769010 & -114.774900 & -114.774166 & -114.773846 & -114.772098 \\
\hline 5.5 & 1.4 & -114.910717 & -114.917041 & -114.916240 & -114.915884 & -114.913968 \\
\hline 5.5 & 1.6 & -114.959771 & -114.966577 & -114.965699 & -114.965305 & -114.964378 \\
\hline 5.5 & 1.7325 & -114.966376 & -114.973528 & -114.972591 & -114.972173 & -114.971316 \\
\hline 5.5 & 1.8 & -114.965037 & -114.972373 & -114.971404 & -114.970973 & -114.970160 \\
\hline 5.5 & 2.0 & -114.949901 & -114.957817 & -114.956743 & -114.956275 & -114.955618 \\
\hline 5.5 & 2.25 & -114.919451 & -114.928182 & -114.926949 & -114.926434 & -114.926018 \\
\hline 5.5 & 2.5 & -114.885945 & -114.895650 & -114.894204 & -114.893637 & -114.893495 \\
\hline 5.5 & 2.75 & -114.854072 & -114.865008 & -114.863263 & -114.862625 & -114.862797 \\
\hline 5.5 & 3.0 & -114.825769 & -114.838347 & -114.836152 & -114.835409 & -114.835970 \\
\hline 5.5 & 3.5 & -114.782168 & -114.800146 & -114.796160 & -114.7 & -114.796685 \\
\hline 5.5 & 4.0 & -114.754756 & -114.782364 & -114.774331 & -114.772353 & -114.775081 \\
\hline 5.5 & 5.0 & -114.730850 & -114.785499 & -114.762279 & -114.758146 & -114.760787 \\
\hline 5.5 & 6.0 & -114.724075 & -114.795749 & -114.760446 & -114.755640 & -114.758339 \\
\hline 5.5 & 8.0 & -114.721175 & -114.796833 & -114.757027 & -114.7 & -114.757800 \\
\hline 6.0 & 1.2 & -114.770179 & -114.775944 & -114.775226 & -114.774912 & -114.773165 \\
\hline 6.0 & 1.4 & -114.911762 & -114.917955 & -114.917170 & -114.916821 & -114.914907 \\
\hline 6.0 & 1.6 & -114.960681 & -114.967349 & -114.966487 & -114.966102 & -114.965173 \\
\hline 6.0 & 1.7325 & -114.967189 & -114.974196 & -114.973277 & -114.972868 & -114.972008 \\
\hline 6.0 & 1.8 & -114.965799 & -114.972987 & -114.972036 & -114.971615 & -114.970798 \\
\hline 6.0 & 2.0 & -114.950505 & -114.958261 & -114.957207 & -114.956750 & -114.956088 \\
\hline 6.0 & 2.25 & -114.919846 & -114.928399 & -114.927188 & -114.926686 & -114.926263 \\
\hline 6.0 & 2.5 & -114.886116 & -114.895614 & -114.894196 & -114.893643 & -114.893499 \\
\hline 6.0 & 2.75 & -114.854000 & -114.864685 & -114.862973 & -114.862351 & -114.862539 \\
\hline 6.0 & 3.0 & -114.825426 & -114.837678 & -114.835529 & -114.834801 & -114.835426 \\
\hline 6.0 & 3.5 & -114.781181 & -114.798504 & -114.794628 & -114.793476 & -114.795497 \\
\hline 6.0 & 4.0 & -114.753014 & -114.779521 & -114.771642 & -114.769563 & -114.773251 \\
\hline 6.0 & 5.0 & -114.727279 & -114.787467 & -114.759999 & -114.753980 & -114.758284 \\
\hline 6.0 & 6.0 & -114.718744 & -114.818382 & -114.763692 & -114.753188 & -114.752837 \\
\hline 6.0 & 8.0 & -114.713429 & -114.854505 & -114.769135 & -114.755073 & -114.755211 \\
\hline 8.0 & 1.2 & -114.770782 & -114.776365 & -114.775668 & -114.775364 & -114.773629 \\
\hline 8.0 & 1.4 & -114.912196 & -114.918195 & -114.917433 & -114.917097 & -114.915178 \\
\hline 8.0 & 1.6 & -114.960942 & -114.967403 & -114.966566 & -114.966195 & -114.965289 \\
\hline 8.0 & 1.7325 & -114.967330 & -114.974122 & -114.973228 & -114.972835 & -114.971997 \\
\hline 8.0 & 1.8 & -114.965878 & -114.972845 & -114.971921 & -114.971516 & -114.970718 \\
\hline 8.0 & 2.0 & -114.950403 & -114.957921 & -114.956897 & -114.956458 & -114.955810 \\
\hline 8.0 & 2.25 & -114.919531 & -114.927820 & -114.926642 & -114.926162 & -114.925749 \\
\hline 8.0 & 2.5 & -114.885608 & -114.894811 & -114.893430 & -114.892901 & -114.892768 \\
\hline 8.0 & 2.75 & -114.853326 & -114.863676 & -114.862008 & -114.861412 & -114.861617 \\
\hline 8.0 & 3.0 & -114.824621 & -114.836485 & -114.834388 & -114.833687 & -114.834350 \\
\hline 8.0 & 3.5 & -114.780268 & -114.796987 & -114.793203 & -114.792070 & -114.794235 \\
\hline
\end{tabular}


Table S1. (continued)

\begin{tabular}{lcccccc}
\hline$R_{\mathrm{Be}-\mathrm{F}}$ & $R_{\mathrm{H}-\mathrm{F}}$ & $\mathrm{CCSD}$ & $\mathrm{CCSD}(\mathrm{T})$ & $\mathrm{R}-\mathrm{CCSD}(\mathrm{T})$ & $\mathrm{CR}-\mathrm{CCSD}(\mathrm{T})$ & $\mathrm{MRCI}(\mathrm{Q})$ \\
\hline 8.0 & 4.0 & -114.752336 & -114.777538 & -114.769979 & -114.767894 & -114.771916 \\
8.0 & 5.0 & -114.728301 & -114.781218 & -114.757272 & -114.751461 & -114.756941 \\
8.0 & 6.0 & -114.721572 & -114.812579 & -114.761629 & -114.750581 & -114.754420 \\
8.0 & 8.0 & -114.718591 & -114.874137 & -114.773514 & -114.754463 & -114.753997 \\
\hline
\end{tabular}

Table S2. The CCSD, CCSD(T), R-CCSD(T), CR-CCSD(T), and MRCI(Q) energies, in hartree, of the BeFH system, as described by the cc-pVTZ basis set, for the Be-F-H angle $\theta=135^{\circ}$. The Be-F and $\mathrm{H}-\mathrm{F}$ bond distances, $R_{\mathrm{Be}-\mathrm{F}}$ and $R_{\mathrm{H}-\mathrm{F}}$, respectively, are in bohr.

\begin{tabular}{|c|c|c|c|c|c|c|}
\hline$R_{\mathrm{Be}-\mathrm{F}}$ & $R_{\mathrm{H}-\mathrm{F}}$ & CCSD & $\operatorname{CCSD}(\mathrm{T})$ & $\mathrm{R}-\mathrm{CCSD}(\mathrm{T})$ & CR-CCSD $(\mathrm{T})$ & $\operatorname{MRCI}(\mathrm{Q})$ \\
\hline 1.8 & 1.2 & -114.342181 & -114.349694 & -114.348970 & -114.348568 & -114.347826 \\
\hline 1.8 & 1.4 & -114.490211 & -114.498254 & -114.497461 & -114.497016 & -114.496324 \\
\hline 1.8 & 1.6 & -114.547277 & -114.556033 & -114.555130 & -114.554637 & -114.553984 \\
\hline 1.8 & 1.7325 & -114.560761 & -114.570253 & -114.569201 & -114.568674 & -114.568280 \\
\hline 1.8 & 1.8 & -114.563797 & -114.573858 & -114.572664 & -114.572116 & -114.571868 \\
\hline 1.8 & 2.0 & -114.568377 & -114.582261 & -114.579723 & -114.578998 & -114.580020 \\
\hline 1.8 & 2.25 & -114.582838 & -114.605167 & -114.598471 & -114.596944 & -114.601320 \\
\hline 1.8 & 2.5 & -114.603288 & -114.627157 & -114.619047 & -114.617666 & -114.622869 \\
\hline 1.8 & 2.75 & -114.620631 & -114.643779 & -114.635212 & -114.634136 & -114.639403 \\
\hline 1.8 & 3.0 & -114.633599 & -114.656630 & -114.647249 & -114.646130 & -114.651236 \\
\hline 1.8 & 3.5 & -114.649516 & -114.673798 & -114.662230 & -114.660572 & -114.665210 \\
\hline 1.8 & 4.0 & -114.657231 & -114.683079 & -114.669512 & -114.667399 & -114.671701 \\
\hline 1.8 & 5.0 & -114.662190 & -114.689813 & -114.673862 & -114.671459 & -114.675568 \\
\hline 1.8 & 6.0 & -114.662985 & -114.690769 & -114.674135 & -114.671867 & -114.676028 \\
\hline 1.8 & 8.0 & -114.663089 & -114.689491 & -114.673533 & -114.671727 & -114.675994 \\
\hline 1.9 & 1.2 & -114.450607 & -114.458173 & -114.457419 & -114.457014 & -114.456241 \\
\hline 1.9 & 1.4 & -114.598365 & -114.606461 & -114.605639 & -114.605191 & -114.604469 \\
\hline 1.9 & 1.6 & -114.654992 & -114.663793 & -114.662864 & -114.662367 & -114.661651 \\
\hline 1.9 & 1.7325 & -114.668039 & -114.677546 & -114.676480 & -114.675948 & -114.675461 \\
\hline 1.9 & 1.8 & -114.670758 & -114.680795 & -114.679603 & -114.679051 & -114.678781 \\
\hline 1.9 & 2.0 & -114.673632 & -114.687084 & -114.684747 & -114.684048 & -114.684751 \\
\hline 1.9 & 2.25 & -114.684994 & -114.707022 & -114.700564 & -114.699043 & -114.703219 \\
\hline 1.9 & 2.5 & -114.703832 & -114.728107 & -114.719874 & -114.718358 & -114.723501 \\
\hline 1.9 & 2.75 & -114.720442 & -114.743809 & -114.735234 & -114.734104 & -114.739358 \\
\hline 1.9 & 3.0 & -114.733001 & -114.755853 & -114.746687 & -114.745637 & -114.750776 \\
\hline 1.9 & 3.5 & -114.748510 & -114.771946 & -114.760984 & -114.759538 & -114.764299 \\
\hline 1.9 & 4.0 & -114.756058 & -114.780663 & -114.767966 & -114.766102 & -114.770581 \\
\hline 1.9 & 5.0 & -114.760927 & -114.786984 & -114.772155 & -114.769991 & -114.774310 \\
\hline 1.9 & 6.0 & -114.761710 & -114.787923 & -114.772435 & -114.770375 & -114.774746 \\
\hline 1.9 & 8.0 & -114.761810 & -114.786908 & -114.771903 & -114.770245 & -114.774707 \\
\hline 2.0 & 1.2 & -114.530598 & -114.538210 & -114.537427 & -114.537019 & -114.536216 \\
\hline 2.0 & 1.4 & -114.678095 & -114.686237 & -114.685386 & -114.684935 & -114.684188 \\
\hline 2.0 & 1.6 & -114.734303 & -114.743142 & -114.742186 & -114.741687 & -114.740930 \\
\hline 2.0 & 1.7325 & -114.746927 & -114.756443 & -114.755362 & -114.754826 & -114.754097 \\
\hline 2.0 & 1.8 & -114.749341 & -114.759350 & -114.758158 & -114.757602 & -114.757276 \\
\hline
\end{tabular}


Table S2. (continued)

\begin{tabular}{|c|c|c|c|c|c|c|}
\hline$R_{\mathrm{Be}-\mathrm{F}}$ & $R_{\mathrm{H}-\mathrm{F}}$ & CCSD & $\operatorname{CCSD}(\mathrm{T})$ & R-CCSD $(\mathrm{T})$ & CR-CCSD(T) & MRCI(Q) \\
\hline 2.0 & 2.0 & -114.750577 & -114.763632 & -114.761471 & -114.760793 & -114.761229 \\
\hline 2.0 & 2.25 & -114.758735 & -114.780364 & -114.774193 & -114.772709 & -114.776678 \\
\hline 2.0 & 2.5 & -114.775792 & -114.800565 & -114.792166 & -114.790507 & -114.795618 \\
\hline 2.0 & 2.75 & -114.791621 & -114.815417 & -114.806720 & -114.805493 & -114.810764 \\
\hline 2.0 & 3.0 & -114.803756 & -114.826674 & -114.817585 & -114.816555 & -114.821748 \\
\hline 2.0 & 3.5 & -114.818845 & -114.841695 & -114.831177 & -114.829907 & -114.834795 \\
\hline 2.0 & 4.0 & -114.826224 & -114.849845 & -114.837844 & -114.836207 & -114.840858 \\
\hline 2.0 & 5.0 & -114.831007 & -114.855754 & -114.841864 & -114.839926 & -114.844450 \\
\hline 2.0 & 6.0 & -114.831780 & -114.856666 & -114.842149 & -114.840285 & -114.844862 \\
\hline 2.0 & 8.0 & -114.831873 & -114.855872 & -114.841678 & -114.840161 & -114.844819 \\
\hline 2.2 & 1.2 & -114.632501 & -114.640183 & -114.639345 & -114.638934 & -114.638128 \\
\hline 2.2 & 1.4 & -114.779502 & -114.787713 & -114.786806 & -114.786352 & -114.785567 \\
\hline 2.2 & 1.6 & -114.834931 & -114.843820 & -114.842815 & -114.842311 & -114.841530 \\
\hline 2.2 & 1.7325 & -114.846768 & -114.856281 & -114.855169 & -114.854628 & -114.853736 \\
\hline 2.2 & 1.8 & -114.848619 & -114.858562 & -114.857364 & -114.856802 & -114.855823 \\
\hline 2.2 & 2.0 & -114.846873 & -114.859230 & -114.857351 & -114.856702 & -114.856851 \\
\hline 2.2 & 2.25 & -114.848478 & -114.868867 & -114.863481 & -114.862162 & -114.865608 \\
\hline 2.2 & 2.5 & -114.861357 & -114.887134 & -114.878421 & -114.876508 & -114.881544 \\
\hline 2.2 & 2.75 & -114.875437 & -114.900578 & -114.891385 & -114.889862 & -114 \\
\hline 2.2 & 3.0 & -114.886681 & -114.910359 & -114.901087 & -114.899957 & -114.905258 \\
\hline 2.2 & 3.5 & -114.900922 & -114.923264 & -114.913244 & -114.912214 & -114.917317 \\
\hline 2.2 & 4.0 & -114.907967 & -114.930275 & -114.919250 & -114.917989 & -114.922933 \\
\hline 2.2 & 5.0 & -114.912583 & -114.9 & -114.922906 & -114.921376 & -114.926253 \\
\hline 2.2 & 6.0 & -114.913340 & -114.936198 & -114.923187 & -114.921682 & -114.926623 \\
\hline 2.2 & 8.0 & -114.913420 & -114.935747 & -114.922816 & -114.921554 & -114.926565 \\
\hline 2.4 & 1.2 & -114.686747 & -114.694472 & -114.693586 & -114.693174 & -114.692746 \\
\hline 2.4 & 1.4 & -114.833279 & $-114 . \varepsilon$ & -114.840576 & -114.840120 & -114.839819 \\
\hline 2.4 & 1.6 & -114.888007 & -114.896915 & -114.895867 & -114.895361 & -114.894604 \\
\hline 2.4 & 1.7325 & -114.899147 & -114.908632 & -114.907491 & -114.906948 & -114.906116 \\
\hline 2.4 & 1.8 & -114.900509 & -114.910372 & -114.909163 & -114.908599 & -114.907658 \\
\hline 2.4 & 2.0 & -114.896231 & -114.908012 & -114.906332 & -114.905695 & -114.905875 \\
\hline 2.4 & 2.25 & -114.891428 & -114.910018 & -114.905633 & -114.904548 & -114.906943 \\
\hline 2.4 & 2.5 & -114.899112 & -114.925394 & -114.916677 & -114.914656 & -114.919514 \\
\hline 2.4 & 2.75 & -114.911043 & -114.937927 & -114.928045 & -114.926157 & -114.931504 \\
\hline 2.4 & 3.0 & -114.921303 & -114.946454 & -114.936630 & -114.935236 & -114.940617 \\
\hline 2.4 & 3.5 & -114.934680 & -114.957321 & -114.947355 & -114.946402 & -114.951662 \\
\hline 2.4 & 4.0 & -114.941401 & -114.963184 & -114.952667 & -114.951662 & -114.956824 \\
\hline 2.4 & 5.0 & -114.945861 & -114.967424 & -114.955916 & -114.954717 & -114.959866 \\
\hline 2.4 & 6.0 & -114.946602 & -114.968156 & -114.956169 & -114.954964 & -114.960189 \\
\hline 2.4 & 8.0 & -114.946665 & -114.967937 & -114.955863 & -114.954816 & -114.960110 \\
\hline 2.5 & 1.2 & -114.703244 & -114.710980 & -114.710075 & -114.709662 & -114.708789 \\
\hline 2.5 & 1.4 & -114.849550 & -114.857811 & -114.856836 & -114.856379 & -114.855590 \\
\hline 2.5 & 1.6 & -114.903952 & -114.912859 & -114.911793 & -114.911286 & -114.910553 \\
\hline 2.5 & 1.7325 & -114.914775 & -114.924238 & -114.923084 & -114.922540 & -114.921762 \\
\hline 2.5 & 1.8 & -114.915919 & -114.925738 & -114.924522 & -114.923958 & -114.923095 \\
\hline 2.5 & 2.0 & -114.910544 & -114.922078 & -114.920470 & -114.919837 & -114.920028 \\
\hline 2.5 & 2.25 & -114.902728 & -114.920307 & -114.916437 & -114.915463 & -114.917265 \\
\hline 2.5 & 2.5 & -114.907391 & -114.933538 & -114.925042 & -114.923054 & -114.927774 \\
\hline 2.5 & 2.75 & -114.918009 & -114.945788 & -114.935562 & -114.933501 & -114.938850 \\
\hline
\end{tabular}


Table S2. (continued)

\begin{tabular}{|c|c|c|c|c|c|c|}
\hline$R_{\mathrm{Be}-\mathrm{F}}$ & $R_{\mathrm{H}-\mathrm{F}}$ & CCSD & $\operatorname{CCSD}(\mathrm{T})$ & $\mathrm{R}-\mathrm{CCSD}(\mathrm{T})$ & CR-CCSD $(\mathrm{T})$ & MRCI(Q) \\
\hline 2.5 & 3.0 & -114.927715 & -114.953819 & -114.943609 & -114.942038 & -114.947456 \\
\hline 2.5 & 3.5 & -114.940644 & -114.963718 & -114.953629 & -114.952651 & -114.957980 \\
\hline 2.5 & 4.0 & -114.947207 & -114.969003 & -114.958583 & -114.957656 & -114.962911 \\
\hline 2.5 & 5.0 & -114.951592 & -114.972807 & -114.961610 & -114.960544 & -114.965812 \\
\hline 2.5 & 6.0 & -114.952325 & -114.973468 & -114.961836 & -114.960757 & -114.966109 \\
\hline 2.5 & 8.0 & -114.952379 & -114.973326 & -114.961550 & -114.960594 & -114.966017 \\
\hline 2.5719 & 1.2 & -114.712178 & -114.719917 & -114.718998 & -114.718587 & -114.717701 \\
\hline 2.5719 & 1.4 & -114.858323 & -114.866585 & -114.865597 & -114.865141 & -114.864361 \\
\hline 2.5719 & 1.6 & -114.912499 & -114.921400 & -114.920322 & -114.919815 & -114.919103 \\
\hline 2.5719 & 1.7325 & -114.923106 & -114.932548 & -114.931386 & -114.930843 & -114.930106 \\
\hline 2.5719 & 1.8 & -114.924103 & -114.933887 & -114.932667 & -114.932103 & -114.931301 \\
\hline 2.5719 & 2.0 & -114.918003 & -114.929376 & -114.927810 & -114.927180 & -114.925669 \\
\hline 2.5719 & 2.25 & -114.908140 & -114.924990 & -114.921471 & -114.920566 & -114.921913 \\
\hline 2.5719 & 2.5 & -114.910466 & -114.936305 & -114.928082 & -114.926160 & -114.930747 \\
\hline 2.5719 & 2.75 & 20005 & -114.948387 & 7943 & 35773 & 1116 \\
\hline 2.5719 & 3.0 & -114.929274 & -114.956132 & -114.945611 & -114.943900 & -114.949342 \\
\hline 2.5719 & 3.5 & -114.941870 & -114.965367 & -114.955131 & -114.954111 & -114.959483 \\
\hline 2.5719 & 4.0 & -114 & -114. & 9825 & -114 & -114 \\
\hline 2.5719 & 5.0 & 2653 & -114 & 2684 & 61699 & 7046 \\
\hline 2.5719 & 6.0 & -114.953380 & -114.974318 & -114.962886 & -114.961886 & -114.967324 \\
\hline 2.5719 & 8.0 & -114.953428 & -114.974217 & -114.962608 & -114.961710 & -114.967222 \\
\hline 2.6 & 1.2 & -114 & -114.722874 & -114 & -114 & -114.720650 \\
\hline 2.6 & 1.4 & 61218 & -114.869480 & 368487 & 868031 & 867255 \\
\hline 2.6 & 1.6 & -114.915307 & -114.924205 & -114.923122 & -114.922616 & -114.921916 \\
\hline 2.6 & 1.7325 & -114.925831 & -114.935266 & -114.934100 & -114.933557 & -114.932836 \\
\hline 2.6 & 1.8 & -114 & -114 & -114 & -114 & 3979 \\
\hline 2.6 & 2.0 & -114.920404 & -114.931718 & -114.930166 & 29537 & -114.928088 \\
\hline 2.6 & 2.25 & -114.909771 & -114.926343 & -114.922954 & -114.922073 & -114.923241 \\
\hline 2.6 & 2.5 & -114.911152 & -114.936819 & -114.928730 & -114.926841 & -114.931369 \\
\hline 2.6 & 2.75 & -114 & -114 & -114 & -114 & -114 \\
\hline 2.6 & 3.0 & -114.929320 & -114.956486 & -114.945837 & -114.944070 & -114.949520 \\
\hline 2.6 & 3.5 & -114.941784 & -114.965470 & -114.955164 & -114.954122 & -114.959510 \\
\hline 2.6 & 4.0 & -114.948190 & -114.970176 & -114.959756 & -114.958871 & -114.964211 \\
\hline 2.6 & 5.0 & -114.952503 & -114.973531 & -114.962547 & -114.961590 & -114.966967 \\
\hline 2.6 & 6.0 & -114.953227 & -114.974105 & -114.962738 & -114.961767 & -114.967237 \\
\hline 2.6 & 8.0 & -114.953273 & -114.974017 & -114.962462 & -114.961586 & -114.967131 \\
\hline 2.7 & 1.2 & -114.723707 & -114.731441 & -114.730504 & -114.730093 & -114.729192 \\
\hline 2.7 & 1.4 & -114.869568 & -114.877823 & -114.876815 & -114.876360 & -114.875593 \\
\hline 2.7 & 1.6 & -114.923355 & -114.932236 & -114.931140 & -114.930635 & -114.929960 \\
\hline 2.7 & 1.7325 & -114.933598 & -114.942997 & -114.941822 & -114.941280 & -114.940610 \\
\hline 2.7 & 1.8 & -114.934353 & -114.944070 & -114.942843 & -114.942281 & -114.941576 \\
\hline 2.7 & 2.0 & -114.927085 & -114.938199 & -114.936692 & -114.936065 & -114.934853 \\
\hline 2.7 & 2.25 & -114.913868 & -114.929489 & -114.926523 & -114.925713 & -114.926281 \\
\hline 2.7 & 2.5 & -114.911760 & -114.936576 & -114.929084 & -114.927352 & -114.931613 \\
\hline 2.7 & 2.75 & -114.919029 & -114.948285 & -114.937582 & -114.935277 & -114.940589 \\
\hline 2.7 & 3.0 & -114.927408 & -114.955705 & -114.944585 & -114.942618 & -114.948094 \\
\hline 2.7 & 3.5 & -114.939377 & -114.963851 & -114.953235 & -114.952092 & -114.957533 \\
\hline 2.7 & 4.0 & -114.945629 & -114.967978 & -114.957462 & -114.956585 & -114.961999 \\
\hline 2.7 & 5.0 & -114.949873 & -114.970865 & -114.960004 & -114.959132 & -114.964610 \\
\hline
\end{tabular}


Table S2. (continued)

\begin{tabular}{|c|c|c|c|c|c|c|}
\hline$R_{\mathrm{Be}-\mathrm{F}}$ & $R_{\mathrm{H}-\mathrm{F}}$ & CCSD & $\operatorname{CCSD}(\mathrm{T})$ & R-CCSD $(\mathrm{T})$ & CR-CCSD(T) & MRCI(Q) \\
\hline 2.7 & 6.0 & -114.950589 & -114.971339 & -114.960150 & -114.959269 & -114.964849 \\
\hline 2.7 & 8.0 & -114.950627 & -114.971281 & -114.959877 & -114.959068 & -114.964728 \\
\hline 2.9 & 1.2 & -114.734512 & -114.742211 & -114.741256 & -114.740848 & -114.741121 \\
\hline 2.9 & 1.4 & -114.879930 & -114.888145 & -114.887118 & -114.886665 & -114.885903 \\
\hline 2.9 & 1.6 & -114.933135 & -114.941959 & -114.940845 & -114.940343 & -114.939703 \\
\hline 2.9 & 1.7325 & -114.942856 & -114.952166 & -114.950978 & -114.950441 & -114.949847 \\
\hline 2.9 & 1.8 & -114.943273 & -114.952872 & -114.951638 & -114.951081 & -114.950490 \\
\hline 2.9 & 2.0 & -114.934462 & -114.945227 & -114.943782 & -114.943162 & -114.942336 \\
\hline 2.9 & 2.25 & -114.916852 & -114.930870 & -114.928536 & -114.927806 & -114.927824 \\
\hline 2.9 & 2.5 & -114.907540 & -114.929704 & -114.923808 & -114.922481 & -114.925884 \\
\hline 2.9 & 2.75 & -114.910155 & -114.939829 & -114.929296 & -114.927002 & -114.932186 \\
\hline 2.9 & 3.0 & -114.916714 & -114.947263 & -114.935237 & -114.932916 & -114.938421 \\
\hline 2.9 & 3.5 & -114.927556 & -114.954101 & -114.942601 & -114.941165 & -114.946689 \\
\hline 2.9 & 4.0 & -114.933499 & -114.957083 & -114.946098 & -114.945139 & -114.950674 \\
\hline 2.9 & 5.0 & -114.937619 & -114.958977 & -114.948103 & -114.947331 & -114.952985 \\
\hline 2.9 & 6.0 & -114.938321 & -114.959198 & -114.948131 & -114.947377 & -114.953157 \\
\hline 2.9 & 8.0 & -114.938346 & -114.959134 & -114.947839 & -114.947130 & -114.953003 \\
\hline 3.1 & 1.2 & -114.740748 & -114.748376 & -114.747417 & -114.747013 & -114.746086 \\
\hline 3.1 & 1.4 & -114.885714 & -114.893854 & -114.892822 & -114.892373 & -114.891616 \\
\hline 3.1 & 1.6 & -114.938358 & -114.947092 & -114.945972 & -114.945475 & -114.944856 \\
\hline 3.1 & 1.7325 & -114.947601 & -114.956796 & -114.955606 & -114.955074 & -114.954535 \\
\hline 3.1 & 1.8 & -114.947721 & -114.957182 & -114.955950 & -114.955400 & -114.954888 \\
\hline 3.1 & 2.0 & -114.937648 & -114.948112 & -114.946708 & -114.946098 & -114 \\
\hline 3.1 & 2.25 & -114.916665 & -114.929511 & -114.927556 & -114.926859 & -114.927645 \\
\hline 3.1 & 2.5 & -114.900654 & -114.919641 & -114.915388 & -114.914406 & -114.916389 \\
\hline 3.1 & 2.75 & -114.897152 & -114.925439 & -114.916099 & -114.914138 & -114.919023 \\
\hline 3.1 & 3.0 & -114.901021 & -114.933257 & -114.920705 & -114.9 & -114.923708 \\
\hline 3.1 & 3.5 & -114.910401 & -114.939598 & -114.926901 & -114.925094 & -114.930674 \\
\hline 3.1 & 4.0 & -114.916027 & -114.941512 & -114.929688 & -114.928532 & -114.934156 \\
\hline 3.1 & 5.0 & -114.920046 & -114.942323 & -114.931112 & -114.930351 & -114.936153 \\
\hline 3.1 & 6.0 & -114.920740 & -114.942217 & -114.930987 & -114.930294 & -114.936249 \\
\hline 3.1 & 8.0 & -114.920756 & -114.942061 & -114.930642 & -114.929992 & -114.936058 \\
\hline 3.3 & 1.2 & -114.745046 & -114.752568 & -114.751617 & -114.751217 & -114.751344 \\
\hline 3.3 & 1.4 & -114.889554 & -114.897582 & -114.896556 & -114.896113 & -114.896260 \\
\hline 3.3 & 1.6 & -114.941654 & -114.950263 & -114.949149 & -114.948659 & -114.948930 \\
\hline 3.3 & 1.7325 & -114.950458 & -114.959508 & -114.958326 & -114.957802 & -114.957301 \\
\hline 3.3 & 1.8 & -114.950317 & -114.959616 & -114.958395 & -114.957852 & -114.957395 \\
\hline 3.3 & 2.0 & -114.939205 & -114.949393 & -114.948023 & -114.947424 & -114.947022 \\
\hline 3.3 & 2.25 & -114.915713 & -114.927724 & -114.925983 & -114.925303 & -114.924480 \\
\hline 3.3 & 2.5 & -114.894276 & -114.910525 & -114.907469 & -114.906660 & -114.907134 \\
\hline 3.3 & 2.75 & -114.883754 & -114.908769 & -114.901528 & -114.900090 & -114.904282 \\
\hline 3.3 & 3.0 & -114.883596 & -114.916027 & -114.903875 & -114.901536 & -114.906958 \\
\hline 3.3 & 3.5 & -114.890857 & -114.923083 & -114.908987 & -114.906811 & -114.912425 \\
\hline 3.3 & 4.0 & -114.896130 & -114.924155 & -114.911127 & -114.909693 & -114.915368 \\
\hline 3.3 & 5.0 & -114.900077 & -114.923801 & -114.911943 & -114.911118 & -114.917037 \\
\hline 3.3 & 6.0 & -114.900774 & -114.923285 & -114.911632 & -114.910944 & -114.917051 \\
\hline 3.3 & 8.0 & -114.900786 & -114.922952 & -114.911202 & -114.910577 & -114.916817 \\
\hline 3.5 & 1.2 & -114.748611 & -114.755993 & -114.755058 & -114.754665 & -114.754682 \\
\hline 3.5 & 1.4 & -114.892666 & -114.900548 & -114.899539 & -114.899103 & -114.898309 \\
\hline
\end{tabular}


Table S2. (continued)

\begin{tabular}{|c|c|c|c|c|c|c|}
\hline$R_{\mathrm{Be}-\mathrm{F}}$ & $R_{\mathrm{H}-\mathrm{F}}$ & CCSD & $\operatorname{CCSD}(\mathrm{T})$ & R-CCSD $(\mathrm{T})$ & CR-CCSD $(\mathrm{T})$ & $\operatorname{MRCI}(\mathrm{Q})$ \\
\hline 3.5 & 1.6 & -114.944250 & -114.952702 & -114.951605 & -114.951122 & -114.951301 \\
\hline 3.5 & 1.7325 & -114.952657 & -114.961533 & -114.960369 & -114.959853 & -114.960124 \\
\hline 3.5 & 1.8 & -114.952286 & -114.961398 & -114.960196 & -114.959663 & -114.959983 \\
\hline 3.5 & 2.0 & -114.940318 & -114.950238 & -114.948901 & -114.948314 & -114.948015 \\
\hline 3.5 & 2.25 & -114.914977 & -114.926370 & -114.924754 & -114.924092 & -114.923687 \\
\hline 3.5 & 2.5 & -114.889598 & -114.903909 & -114.901541 & -114.900791 & -114.901489 \\
\hline 3.5 & 2.75 & -114.872390 & -114.893358 & -114.888317 & -114.887300 & -114.890065 \\
\hline 3.5 & 3.0 & -114.866674 & -114.896995 & -114.886574 & -114.884713 & -114.889917 \\
\hline 3.5 & 3.5 & -114.870600 & -114.905809 & -114.890375 & -114.887943 & -114.893573 \\
\hline 3.5 & 4.0 & -114.875423 & -114.906566 & -114.891994 & -114.890243 & -114.895935 \\
\hline 3.5 & 5.0 & -114.879331 & -114.905025 & -114.892209 & -114.891257 & -114.897258 \\
\hline 3.5 & 6.0 & -114.880050 & -114.904007 & -114.891681 & -114.890950 & -114.897184 \\
\hline 3.5 & 8.0 & -114.880068 & -114.903410 & -114.891139 & -114.890507 & -114.896902 \\
\hline 3.7 & 1.2 & -114.751893 & -114.759108 & -114.758197 & -114.757811 & -114.757702 \\
\hline 3.7 & 1.4 & -114.895523 & -114.903231 & -114.902245 & -114.901817 & -114.900961 \\
\hline 3.7 & 1.6 & -114.946634 & -114.954900 & -114.953827 & -114.953353 & -114.953429 \\
\hline 3.7 & 1.7325 & -114.954686 & -114.963364 & -114.962225 & -114.961719 & -114.961894 \\
\hline 3.7 & 1.8 & -114.954117 & -114.963020 & -114.961844 & -114.961322 & -114.961545 \\
\hline 3.7 & 2.0 & -114.941444 & -114.951098 & -114.949795 & -114.949222 & -114.949580 \\
\hline 3.7 & 2.25 & -114.914736 & -114.925643 & -114.924109 & -114.923466 & -114.923307 \\
\hline 3.7 & 2.5 & -114.886663 & -114.899693 & -114.897682 & -114.896960 & -114.896440 \\
\hline 3.7 & 2.75 & -114.864183 & -114.881739 & -114.878247 & -114.877413 & -114.878231 \\
\hline 3.7 & 3.0 & -114.852051 & -114.878210 & -114.870483 & -114.869189 & -114.873714 \\
\hline 3.7 & 3.5 & -114.850678 & -114.888048 & -114.871851 & -114.869397 & -114.875048 \\
\hline 3.7 & 4.0 & -114.854813 & -114.889494 & -114.873130 & -114.871088 & -114.876762 \\
\hline 3.7 & 5.0 & -114.858709 & -114.886900 & -114.872800 & -114.871680 & -114.877720 \\
\hline 3.7 & 6.0 & -114.859476 & -114.885289 & -114.872036 & -114.871227 & -114.877554 \\
\hline 3.7 & 8.0 & -114.859514 & -114.884338 & -114.871357 & -114.870697 & -114.877223 \\
\hline 3.9 & 1.2 & -114.754990 & -114.762021 & -114.761136 & -114.760759 & -114.760471 \\
\hline 3.9 & 1.4 & -114.898235 & -114.905753 & -114.904794 & -114.904375 & -114.904191 \\
\hline 3.9 & 1.6 & -114.948925 & -114.956992 & -114.955946 & -114.955483 & -114.955447 \\
\hline 3.9 & 1.7325 & -114.956671 & -114.965137 & -114.964027 & -114.963532 & -114.963603 \\
\hline 3.9 & 1.8 & -114.955933 & -114.964616 & -114.963469 & -114.962959 & -114.963084 \\
\hline 3.9 & 2.0 & -114.942684 & -114.952076 & -114.950808 & -114.950248 & -114.950522 \\
\hline 3.9 & 2.25 & -114.914959 & -114.925463 & -114.923989 & -114.923365 & -114.923771 \\
\hline 3.9 & 2.5 & -114.885083 & -114.897250 & -114.895430 & -114.894731 & -114.894665 \\
\hline 3.9 & 2.75 & -114.859001 & -114.874258 & -114.871600 & -114.870818 & -114.871452 \\
\hline 3.9 & 3.0 & -114.841000 & -114.862596 & -114.857375 & -114.856426 & -114.859280 \\
\hline 3.9 & 3.5 & -114.831910 & -114.869524 & -114.853866 & -114.851698 & -114.857398 \\
\hline 3.9 & 4.0 & -114.834829 & -114.873115 & -114.854954 & -114.852729 & -114.858366 \\
\hline 3.9 & 5.0 & -114.838701 & -114.869932 & -114.854211 & -114.852904 & -114.858935 \\
\hline 3.9 & 6.0 & -114.839552 & -114.867653 & -114.853202 & -114.852291 & -114.858675 \\
\hline 3.9 & 8.0 & -114.839633 & -114.866255 & -114.852367 & -114.851663 & -114.858293 \\
\hline 4.1 & 1.2 & -114.757859 & -114.764703 & -114.763845 & -114.763477 & -114.761737 \\
\hline 4.1 & 1.4 & -114.900769 & -114.908093 & -114.907162 & -114.906753 & -114.904828 \\
\hline 4.1 & 1.6 & -114.951098 & -114.958960 & -114.957942 & -114.957490 & -114.957342 \\
\hline 4.1 & 1.7325 & -114.958583 & -114.966835 & -114.965753 & -114.965270 & -114.965236 \\
\hline 4.1 & 1.8 & -114.957704 & -114.966165 & -114.965049 & -114.964551 & -114.964575 \\
\hline 4.1 & 2.0 & -114.943988 & -114.953127 & -114.951893 & -114.951348 & -114.951542 \\
\hline
\end{tabular}


Table S2. (continued)

\begin{tabular}{|c|c|c|c|c|c|c|}
\hline$R_{\mathrm{Be}-\mathrm{F}}$ & $R_{\mathrm{H}-\mathrm{F}}$ & CCSD & $\operatorname{CCSD}(\mathrm{T})$ & R-CCSD $(\mathrm{T})$ & CR-CCSD(T) & $\operatorname{MRCI}(\mathrm{Q})$ \\
\hline 4.1 & 2.25 & -114.915501 & -114.925660 & -114.924235 & -114.923630 & -114.923983 \\
\hline 4.1 & 2.5 & -114.884416 & -114.895970 & -114.894260 & -114.893585 & -114.893908 \\
\hline 4.1 & 2.75 & -114.856058 & -114.869878 & -114.867624 & -114.866867 & -114.867088 \\
\hline 4.1 & 3.0 & -114.833761 & -114.851892 & -114.848230 & -114.847381 & -114.847851 \\
\hline 4.1 & 3.5 & -114.815179 & -114.850249 & -114.836918 & -114.835267 & -114.840972 \\
\hline 4.1 & 4.0 & -114.815836 & -114.857131 & -114.837660 & -114.835433 & -114.841044 \\
\hline 4.1 & 5.0 & -114.819571 & -114.854373 & -114.836703 & -114.835221 & -114.841192 \\
\hline 4.1 & 6.0 & -114.820542 & -114.851401 & -114.835460 & -114.834436 & -114.840836 \\
\hline 4.1 & 8.0 & -114.820696 & -114.849469 & -114.834455 & -114.833696 & -114.840406 \\
\hline 4.5 & 1.2 & -114.762664 & -114.769162 & -114.768351 & -114.768000 & -114.766248 \\
\hline 4.5 & 1.4 & -114.905056 & -114.912019 & -114.911136 & -114.910747 & -114.908814 \\
\hline 4.5 & 1.6 & -114.954827 & -114.962309 & -114.961343 & -114.960912 & -114.960557 \\
\hline 4.5 & 1.7325 & -114.961918 & -114.969773 & -114.968745 & -114.968285 & -114.968051 \\
\hline 4.5 & 1.8 & -114.960829 & -114.968883 & -114.967821 & -114.967347 & -114.967177 \\
\hline 4.5 & 2.0 & -114.946441 & -114.955130 & -114.953955 & -114.953438 & -114.953467 \\
\hline 4.5 & 2.25 & -114.916949 & -114.926551 & -114.925202 & -114.924630 & -114.924902 \\
\hline 4.5 & 2.5 & -114.884475 & -114.895214 & -114.893628 & -114.892995 & -114.893453 \\
\hline 4.5 & 2.75 & -114.853843 & -114.866141 & -114.864198 & -114.863487 & 63965 \\
\hline 4.5 & 3.0 & -114.827300 & -114.841982 & -114.839420 & -114.838605 & 41351 \\
\hline 4.5 & 3.5 & -114.791952 & -114.817235 & -114.810472 & -114.809425 & -114.812902 \\
\hline 4.5 & 4.0 & -114.782464 & -114.823964 & -114.806273 & -114.804616 & -114.810237 \\
\hline 4.5 & 5.0 & -114.784420 & -114.827613 & -114.805451 & -114 & -114 \\
\hline 4.5 & 6.0 & -114.785696 & -114.823707 & -114.803829 & -114.802595 & -114.808836 \\
\hline 4.5 & 8.0 & -114.786099 & -114.820516 & -114.802450 & -114.801568 & -114.808320 \\
\hline 4.7 & 1.2 & -114.764541 & -114.770890 & -114.770099 & -114.769756 & -114.768002 \\
\hline 4.7 & 1.4 & -114.906740 & -114. & -114.912686 & -114.912305 & -114 \\
\hline 4.7 & 1.6 & -114.956306 & -114.963624 & -114.962680 & -114.962258 & -114.960167 \\
\hline 4.7 & 1.7325 & -114.963254 & -114.970938 & -114.969932 & -114.969483 & -114.969162 \\
\hline 4.7 & 1.8 & -114.962089 & -114.969969 & -114.968930 & -114.968466 & -114.968211 \\
\hline 4.7 & 2.0 & -114.947467 & -114.955966 & -114.9 & -114. & -114.954263 \\
\hline 4.7 & 2.25 & -114.917649 & -114.927030 & -114.925711 & -114.925153 & -114.925370 \\
\hline 4.7 & 2.5 & -114.884778 & -114.895233 & -114.893687 & -114.893070 & -114.893531 \\
\hline 4.7 & 2.75 & -114.853596 & -114.865465 & -114.863589 & -114.862897 & -114.863517 \\
\hline 4.7 & 3.0 & -114.826165 & -114.840056 & -114.837658 & -114.836861 & -114.837537 \\
\hline 4.7 & 3.5 & -114.786252 & -114.808117 & -114.803036 & -114.801940 & -114.803203 \\
\hline 4.7 & 4.0 & -114.769524 & -114.807193 & -114.793064 & -114.791647 & -114.796962 \\
\hline 4.7 & 5.0 & -114.768577 & -114.816120 & -114.791920 & -114.790158 & -114.795451 \\
\hline 4.7 & 6.0 & -114.769918 & -114.812504 & -114.790167 & -114.788826 & -114.794811 \\
\hline 4.7 & 8.0 & -114.770478 & -114.808672 & -114.788556 & -114.787600 & -114.794262 \\
\hline 5.0 & 1.2 & -114.766730 & -114.772894 & -114.772127 & -114.771793 & -114.770038 \\
\hline 5.0 & 1.4 & -114.908708 & -114.915321 & -114.914484 & -114.914113 & -114.912183 \\
\hline 5.0 & 1.6 & -114.958040 & -114.965152 & -114.964234 & -114.963824 & -114.962994 \\
\hline 5.0 & 1.7325 & -114.964824 & -114.972295 & -114.971317 & -114.970880 & -114.970148 \\
\hline 5.0 & 1.8 & -114.963575 & -114.971236 & -114.970225 & -114.969775 & -114.969101 \\
\hline 5.0 & 2.0 & -114.948694 & -114.956956 & -114.955837 & -114.955348 & -114.954882 \\
\hline 5.0 & 2.25 & -114.918530 & -114.927643 & -114.926359 & -114.925819 & -114.925954 \\
\hline 5.0 & 2.5 & -114.885269 & -114.895398 & -114.893896 & -114.893300 & -114.893726 \\
\hline 5.0 & 2.75 & -114.853602 & -114.865029 & -114.863216 & -114.862548 & -114.862925 \\
\hline 5.0 & 3.0 & -114.825486 & -114.838675 & -114.836395 & -114.835620 & -114.836305 \\
\hline
\end{tabular}


Table S2. (continued)

\begin{tabular}{|c|c|c|c|c|c|c|}
\hline$R_{\mathrm{Be}-\mathrm{F}}$ & $R_{\mathrm{H}-\mathrm{F}}$ & CCSD & $\operatorname{CCSD}(\mathrm{T})$ & $\mathrm{R}-\mathrm{CCSD}(\mathrm{T})$ & CR-CCSD $(\mathrm{T})$ & $\operatorname{MRCI}(\mathrm{Q})$ \\
\hline 5.0 & 3.5 & -114.782464 & -114.801807 & -114.797573 & -114.796411 & -114.797690 \\
\hline 5.0 & 4.0 & -114.757524 & -114.788880 & -114.779392 & -114.777723 & -114.778450 \\
\hline 5.0 & 5.0 & -114.747644 & -114.800742 & -114.774978 & -114.772962 & -114.777173 \\
\hline 5.0 & 6.0 & -114.748453 & -114.799664 & -114.773029 & -114.771359 & -114.776391 \\
\hline 5.0 & 8.0 & -114.749125 & -114.795083 & -114.770862 & -114.769673 & -114.775816 \\
\hline 5.2 & 1.2 & -114.767827 & -114.773890 & -114.773136 & -114.772807 & -114.771052 \\
\hline 5.2 & 1.4 & -114.909694 & -114.916201 & -114.915377 & -114.915012 & -114.913084 \\
\hline 5.2 & 1.6 & -114.958908 & -114.965908 & -114.965005 & -114.964601 & -114.963735 \\
\hline 5.2 & 1.7325 & -114.965611 & -114.972966 & -114.972003 & -114.971573 & -114.970797 \\
\hline 5.2 & 1.8 & -114.964320 & -114.971862 & -114.970867 & -114.970424 & -114.969702 \\
\hline 5.2 & 2.0 & -114.949313 & -114.957448 & -114.956346 & -114.955865 & -114.955328 \\
\hline 5.2 & 2.25 & -114.918987 & -114.927957 & -114.926692 & -114.926162 & -114.925897 \\
\hline 5.2 & 2.5 & -114.885555 & -114.895518 & -114.894037 & -114.893452 & -114.893479 \\
\hline 5.2 & 2.75 & -114.853696 & -114.864913 & -114.863129 & -114.862472 & -114.862809 \\
\hline 5.2 & 3.0 & -114.825344 & -114.838238 & -114.836000 & -114.835238 & -114.835936 \\
\hline 5.2 & 3.5 & -114.781499 & -114.800036 & -114.795970 & -114.794799 & -114.796431 \\
\hline 5.2 & 4.0 & -114.754136 & -114.783304 & -114.774880 & -114.772951 & -114.775008 \\
\hline 5.2 & 5.0 & -114.736663 & -114.792468 & -114.766895 & -114.764195 & -114.764591 \\
\hline 5.2 & 6.0 & -114.736029 & -114.794892 & -114.764772 & -114.762467 & -114.763931 \\
\hline 5.2 & 8.0 & -114.736423 & -114.790117 & -114.761937 & -114.760306 & -114.763567 \\
\hline 5.5 & 1.2 & -114.769036 & -114.774978 & -114.774238 & -114.773916 & -114.772161 \\
\hline 5.5 & 1.4 & -114.910777 & -114.917156 & -114.916348 & -114.915991 & -114.914065 \\
\hline 5.5 & 1.6 & -114.959859 & -114.966725 & -114.965838 & -114.965443 & -114.964544 \\
\hline 5.5 & 1.7325 & -114.966473 & -114.973687 & -114.972741 & -114.972321 & -114.971506 \\
\hline 5.5 & 1.8 & -114.965135 & -114.972534 & -114.971556 & -114.971123 & -114.970358 \\
\hline 5.5 & 2.0 & -114.949988 & -114.957970 & -114.956887 & -114.956416 & -114.955827 \\
\hline 5.5 & 2.25 & -114.919489 & -114.928289 & -114.927045 & -114.926527 & -114.926201 \\
\hline 5.5 & 2.5 & -114.885882 & -114.895651 & -114.894195 & -114.893624 & -114.893592 \\
\hline 5.5 & 2.75 & -114.853843 & -114.864827 & -114.863074 & -114.862432 & -114.862730 \\
\hline 5.5 & 3.0 & -114.825298 & -114.837887 & -114.835691 & -114.834943 & -114.835649 \\
\hline 5.5 & 3.5 & -114.780947 & -114.798803 & -114.794844 & -114.793673 & -114.795604 \\
\hline 5.5 & 4.0 & -114.752470 & -114.780236 & -114.772083 & -114.769979 & -114.773251 \\
\hline 5.5 & 5.0 & -114.727542 & -114.787346 & -114.761104 & -114.756306 & -114.759427 \\
\hline 5.5 & 6.0 & -114.722500 & -114.798597 & -114.760003 & -114.754937 & -114.758127 \\
\hline 5.5 & 8.0 & -114.721032 & -114.795340 & -114.756198 & -114.752390 & -114.757805 \\
\hline 6.0 & 1.2 & -114.770210 & -114.776008 & -114.775286 & -114.774971 & -114.773219 \\
\hline 6.0 & 1.4 & -114.911817 & -114.918045 & -114.917256 & -114.916906 & -114.914985 \\
\hline 6.0 & 1.6 & -114.960758 & -114.967465 & -114.966598 & -114.966212 & -114.965296 \\
\hline 6.0 & 1.7325 & -114.967276 & -114.974325 & -114.973400 & -114.972989 & -114.972150 \\
\hline 6.0 & 1.8 & -114.965889 & -114.973119 & -114.972163 & -114.971740 & -114.970945 \\
\hline 6.0 & 2.0 & -114.950598 & -114.958400 & -114.957339 & -114.956881 & -114.956236 \\
\hline 6.0 & 2.25 & -114.919925 & -114.928526 & -114.927308 & -114.926804 & -114.926398 \\
\hline 6.0 & 2.5 & -114.886155 & -114.895702 & -114.894275 & -114.893720 & -114.893593 \\
\hline 6.0 & 2.75 & -114.853969 & -114.864697 & -114.862978 & -114.862353 & -114.862558 \\
\hline 6.0 & 3.0 & -114.825292 & -114.837575 & -114.835419 & -114.834689 & -114.835330 \\
\hline 6.0 & 3.5 & -114.780754 & -114.798052 & -114.794176 & -114.793012 & -114.795077 \\
\hline 6.0 & 4.0 & -114.752242 & -114.778836 & -114.770850 & -114.768700 & -114.772506 \\
\hline 6.0 & 5.0 & -114.725939 & -114.790973 & -114.760174 & -114.753275 & -114.757426 \\
\hline 6.0 & 6.0 & -114.717410 & -114.828224 & -114.765490 & -114.753375 & -114.755514 \\
\hline
\end{tabular}


Table S2. (continued)

\begin{tabular}{lcccccc}
\hline$R_{\mathrm{Be}-\mathrm{F}}$ & $R_{\mathrm{H}-\mathrm{F}}$ & $\mathrm{CCSD}$ & $\mathrm{CCSD}(\mathrm{T})$ & $\mathrm{R}-\mathrm{CCSD}(\mathrm{T})$ & $\mathrm{CR}-\mathrm{CCSD}(\mathrm{T})$ & $\mathrm{MRCI}(\mathrm{Q})$ \\
\hline 6.0 & 8.0 & -114.712884 & -114.857699 & -114.769530 & -114.755158 & -114.755215 \\
8.0 & 1.2 & -114.770832 & -114.776420 & -114.775722 & -114.775419 & -114.773681 \\
8.0 & 1.4 & -114.912256 & -114.918261 & -114.917498 & -114.917161 & -114.915259 \\
8.0 & 1.6 & -114.961014 & -114.967483 & -114.966645 & -114.966273 & -114.965368 \\
8.0 & 1.7325 & -114.967411 & -114.974210 & -114.973316 & -114.972922 & -114.972083 \\
8.0 & 1.8 & -114.965963 & -114.972938 & -114.972013 & -114.971607 & -114.970810 \\
8.0 & 2.0 & -114.950498 & -114.958026 & -114.957000 & -114.956561 & -114.955914 \\
8.0 & 2.25 & -114.919635 & -114.927936 & -114.926756 & -114.926275 & -114.925863 \\
8.0 & 2.5 & -114.885717 & -114.894934 & -114.893551 & -114.893021 & -114.892888 \\
8.0 & 2.75 & -114.853434 & -114.863802 & -114.862130 & -114.861534 & -114.861737 \\
8.0 & 3.0 & -114.824726 & -114.836609 & -114.834508 & -114.833807 & -114.834467 \\
8.0 & 3.5 & -114.780354 & -114.797100 & -114.793309 & -114.792175 & -114.794333 \\
8.0 & 4.0 & -114.752402 & -114.777642 & -114.770073 & -114.767979 & -114.771992 \\
8.0 & 5.0 & -114.728381 & -114.781209 & -114.757349 & -114.751493 & -114.756993 \\
8.0 & 6.0 & -114.721674 & -114.814042 & -114.762100 & -114.750730 & -114.754471 \\
8.0 & 8.0 & -114.718613 & -114.880153 & -114.774806 & -114.754874 & -114.753932 \\
\hline
\end{tabular}

Table S3. The CCSD, CCSD(T), R-CCSD $(\mathrm{T})$, CR-CCSD $(\mathrm{T})$, and MRCI(Q) energies, in hartree, of the BeFH system, as described by the cc-pVTZ basis set, for the Be-F-H angle $\theta=90^{\circ}$. The Be $-\mathrm{F}$ and $\mathrm{H}-\mathrm{F}$ bond distances, $R_{\mathrm{Be}-\mathrm{F}}$ and $R_{\mathrm{H}-\mathrm{F}}$, respectively, are in bohr.

\begin{tabular}{lcccccc}
\hline$R_{\mathrm{Be}-\mathrm{F}}$ & $R_{\mathrm{H}-\mathrm{F}}$ & $\mathrm{CCSD}$ & $\mathrm{CCSD}(\mathrm{T})$ & $\mathrm{R}-\mathrm{CCSD}(\mathrm{T})$ & $\mathrm{CR}-\mathrm{CCSD}(\mathrm{T})$ & $\mathrm{MRCI}(\mathrm{Q})$ \\
\hline 1.8 & 1.2 & -114.300818 & -114.308522 & -114.307794 & -114.307380 & -114.306937 \\
1.8 & 1.4 & -114.460547 & -114.468874 & -114.468082 & -114.467619 & -114.467348 \\
1.8 & 1.6 & -114.533563 & -114.542690 & -114.541803 & -114.541282 & -114.541289 \\
1.8 & 1.7325 & -114.559670 & -114.569454 & -114.568473 & -114.567907 & -114.568195 \\
1.8 & 1.8 & -114.569459 & -114.579615 & -114.578572 & -114.577982 & -114.578441 \\
1.8 & 2.0 & -114.591799 & -114.603104 & -114.601824 & -114.601156 & -114.602167 \\
1.8 & 2.25 & -114.614391 & -114.626755 & -114.625130 & -114.624398 & -114.626054 \\
1.8 & 2.5 & -114.633660 & -114.646375 & -114.644459 & -114.643733 & -114.645780 \\
1.8 & 2.75 & -114.648681 & -114.661353 & -114.659194 & -114.658501 & -114.660729 \\
1.8 & 3.0 & -114.659289 & -114.671845 & -114.669450 & -114.668779 & -114.671091 \\
1.8 & 3.5 & -114.670043 & -114.682650 & -114.679677 & -114.679002 & -114.681448 \\
1.8 & 4.0 & -114.672327 & -114.685559 & -114.681738 & -114.681003 & -114.683676 \\
1.8 & 5.0 & -114.668886 & -114.684927 & -114.678399 & -114.677419 & -114.680699 \\
1.8 & 6.0 & -114.665560 & -114.685274 & -114.675372 & -114.674110 & -114.677862 \\
1.8 & 8.0 & -114.663457 & -114.687641 & -114.673493 & -114.672005 & -114.676184 \\
1.9 & 1.2 & -114.413885 & -114.421662 & -114.420899 & -114.420481 & -114.420014 \\
1.9 & 1.4 & -114.571946 & -114.580352 & -114.579523 & -114.579056 & -114.578767 \\
1.9 & 1.6 & -114.642728 & -114.651943 & -114.651018 & -114.650492 & -114.650482 \\
1.9 & 1.7325 & -114.667067 & -114.676957 & -114.675936 & -114.675364 & -114.675636 \\
1.9 & 1.8 & -114.675890 & -114.686169 & -114.685084 & -114.684486 & -114.684939 \\
1.9 & 2.0 & -114.695327 & -114.706855 & -114.705517 & -114.704834 & -114.705887 \\
1.9 & 2.25 & -114.714920 & -114.727718 & -114.725987 & -114.725223 & -114.726997 \\
1.9 & 2.5 & -114.732319 & -114.745613 & -114.743540 & -114.742772 & -114.745009 \\
\hline & & & & & &
\end{tabular}


Table S3. (continued)

\begin{tabular}{|c|c|c|c|c|c|c|}
\hline$R_{\mathrm{Be}-\mathrm{F}}$ & $R_{\mathrm{H}-\mathrm{F}}$ & CCSD & $\operatorname{CCSD}(\mathrm{T})$ & R-CCSD $(\mathrm{T})$ & CR-CCSD(T) & MRCI(Q) \\
\hline 1.9 & 2.75 & -114.746310 & -114.759604 & -114.757252 & -114.756521 & -114.758971 \\
\hline 1.9 & 3.0 & -114.756394 & -114.769567 & -114.766950 & -114.766249 & -114.768794 \\
\hline 1.9 & 3.5 & -114.766932 & -114.780104 & -114.776866 & -114.776168 & -114.778844 \\
\hline 1.9 & 4.0 & -114.769515 & -114.783246 & -114.779132 & -114.778379 & -114.781269 \\
\hline 1.9 & 5.0 & -114.766876 & -114.783175 & -114.776420 & -114.775439 & -114.778909 \\
\hline 1.9 & 6.0 & -114.764008 & -114.783558 & -114.773700 & -114.772470 & -114.776406 \\
\hline 1.9 & 8.0 & -114.762145 & -114.785505 & -114.771919 & -114.770515 & -114.774885 \\
\hline 2.0 & 1.2 & -114.498135 & -114.505977 & -114.505180 & -114.504758 & -114.504270 \\
\hline 2.0 & 1.4 & -114.654675 & -114.663151 & -114.662286 & -114.661815 & -114.661503 \\
\hline 2.0 & 1.6 & -114.723398 & -114.732691 & -114.731728 & -114.731196 & -114.731163 \\
\hline 2.0 & 1.7325 & -114.746083 & -114.756062 & -114.755003 & -114.754424 & -114.754675 \\
\hline 2.0 & 1.8 & -114.753989 & -114.764369 & -114.763245 & -114.762639 & -114.763079 \\
\hline 2.0 & 2.0 & -114.770600 & -114.782319 & -114.780929 & -114.780233 & -114.781315 \\
\hline 2.0 & 2.25 & -114.787156 & -114.800363 & -114.798530 & -114.797733 & -114.799621 \\
\hline 2.0 & 2.5 & -114.802615 & -114.816495 & -114.814259 & -114.813447 & -114.815880 \\
\hline 2.0 & 2.75 & -114.815534 & -114.829473 & -114.826916 & -114.826145 & -114.828830 \\
\hline 2.0 & 3.0 & -114.825069 & -114.838882 & -114.836031 & -114.835298 & -114.838092 \\
\hline 2.0 & 3.5 & -114.835345 & -114.849101 & -114.845584 & -114.844866 & -114.847791 \\
\hline 2.0 & 4.0 & -114.838184 & -114.8 & -114.848009 & -114.847241 & -114.850366 \\
\hline 2.0 & 5.0 & -114.836281 & -114.852835 & -114.845861 & -114.844883 & -114.848558 \\
\hline 2.0 & 6.0 & -114.833828 & -114.853240 & -114.843422 & -114.842229 & -114.846360 \\
\hline 2.0 & 8.0 & -114.832181 & -114.854833 & -114.841740 & -114.840418 & -114.844984 \\
\hline 2.2 & 1.2 & -114.607562 & -114.615516 & -114.614652 & -114.614225 & -114.613707 \\
\hline 2.2 & 1.4 & -114.761450 & -114.770044 & -114.769109 & -114.768631 & -114.768277 \\
\hline 2.2 & 1.6 & -114.826541 & -114.835954 & -114.834920 & -114.834380 & -114.834302 \\
\hline 2.2 & 1.7325 & -114.846253 & -114.856361 & -114.855230 & -114.854642 & -114.854844 \\
\hline 2.2 & 1.8 & -114.852480 & -114.863001 & -114.861805 & -114.861190 & -114.861587 \\
\hline 2.2 & 2.0 & -114.863728 & -114.875715 & -114.874239 & -114.873526 & -114.874642 \\
\hline 2.2 & 2.25 & -114.874124 & -114.888040 & -114.886024 & -114.885174 & -114.887266 \\
\hline 2.2 & 2.5 & -114.885487 & -114.900524 & -114.897960 & -114.897052 & -114.899843 \\
\hline 2.2 & 2.75 & -114.896136 & -114.911415 & -114.908424 & -114.907556 & -114.910710 \\
\hline 2.2 & 3.0 & -114.904500 & -114.919659 & -114.916306 & -114.915498 & -114.918802 \\
\hline 2.2 & 3.5 & -114.914135 & -114.929099 & -114.924997 & -114.924238 & -114.927674 \\
\hline 2.2 & 4.0 & -114.917358 & -114.932623 & -114.927599 & -114.926809 & -114.930413 \\
\hline 2.2 & 5.0 & -114.916709 & -114.933762 & -114.926379 & -114.925425 & -114.929509 \\
\hline 2.2 & 6.0 & -114.914966 & -114.934181 & -114.924427 & -114.923320 & -114.927829 \\
\hline 2.2 & 8.0 & -114.913683 & -114.935238 & -114.922938 & -114.921775 & -114.926706 \\
\hline 2.4 & 1.2 & -114.668067 & -114.676105 & -114.675182 & -114.674752 & -114.674176 \\
\hline 2.4 & 1.4 & -114.819757 & -114.828434 & -114.827437 & -114.826955 & -114.826605 \\
\hline 2.4 & 1.6 & -114.881812 & -114.891302 & -114.890204 & -114.889660 & -114.889575 \\
\hline 2.4 & 1.7325 & -114.898993 & -114.909166 & -114.907973 & -114.907381 & -114.907550 \\
\hline 2.4 & 1.8 & -114.903760 & -114.914342 & -114.913088 & -114.912468 & -114.912815 \\
\hline 2.4 & 2.0 & -114.910116 & -114.922211 & -114.920680 & -114.919962 & -114.921030 \\
\hline 2.4 & 2.25 & -114.914304 & -114.928709 & -114.926566 & -114.925684 & -114.927759 \\
\hline 2.4 & 2.5 & -114.921210 & -114.937319 & -114.934448 & -114.933448 & -114.936501 \\
\hline 2.4 & 2.75 & -114.929370 & -114.946032 & -114.942588 & -114.941606 & -114.945173 \\
\hline 2.4 & 3.0 & -114.936459 & -114.953047 & -114.949158 & -114.948257 & -114.952028 \\
\hline 2.4 & 3.5 & -114.945309 & -114.961535 & -114.956825 & -114.956025 & -114.959936 \\
\hline 2.4 & 4.0 & -114.948757 & -114.965059 & -114.959431 & -114.958629 & -114.962673 \\
\hline
\end{tabular}


Table S3. (continued)

\begin{tabular}{|c|c|c|c|c|c|c|}
\hline$R_{\mathrm{Be}-\mathrm{F}}$ & $R_{\mathrm{H}-\mathrm{F}}$ & CCSD & $\operatorname{CCSD}(\mathrm{T})$ & $\mathrm{R}-\mathrm{CCSD}(\mathrm{T})$ & CR-CCSD $(\mathrm{T})$ & $\operatorname{MRCI}(\mathrm{Q})$ \\
\hline 2.4 & 5.0 & -114.949076 & -114.966632 & -114.958878 & -114.957964 & -114.962414 \\
\hline 2.4 & 6.0 & -114.947894 & -114.967039 & -114.957319 & -114.956307 & -114.961144 \\
\hline 2.4 & 8.0 & -114.946896 & -114.967749 & -114.956010 & -114.954992 & -114.960228 \\
\hline 2.5 & 1.2 & -114.687237 & -114.695306 & -114.694358 & -114.693927 & -114.693117 \\
\hline 2.5 & 1.4 & -114.837972 & -114.846678 & -114.845654 & -114.845171 & -114.844737 \\
\hline 2.5 & 1.6 & -114.898710 & -114.908220 & -114.907095 & -114.906550 & -114.906429 \\
\hline 2.5 & 1.7325 & -114.914781 & -114.924964 & -114.923746 & -114.923153 & -114.923270 \\
\hline 2.5 & 1.8 & -114.918900 & -114.929486 & -114.928207 & -114.927587 & -114.927871 \\
\hline 2.5 & 2.0 & -114.923011 & -114.935104 & -114.933556 & -114.932839 & -114.933857 \\
\hline 2.5 & 2.25 & -114.924110 & -114.938651 & -114.936475 & -114.935588 & -114.937636 \\
\hline 2.5 & 2.5 & -114.928616 & -114.945191 & -114.942192 & -114.941153 & -114.944299 \\
\hline 2.5 & 2.75 & -114.935414 & -114.952767 & -114.949098 & -114.948054 & -114.951813 \\
\hline 2.5 & 3.0 & -114.941813 & -114.959145 & -114.954979 & -114.954022 & -114.958024 \\
\hline 2.5 & 3.5 & -114.950219 & -114.967100 & -114.962080 & -114.961255 & -114.965403 \\
\hline 2.5 & 4.0 & -114.953727 & -114.970555 & 4629 & 3823 & 68086 \\
\hline 2.5 & 5.0 & -114.954430 & -114.972249 & -114.964320 & -114.963430 & -114.968059 \\
\hline 2.5 & 6.0 & -114.953478 & -114.972646 & -114.962924 & -114.961960 & -114.966955 \\
\hline 2.5 & 8.0 & 2597 & -114 & 1699 & 745 & 6125 \\
\hline 2.5719 & 1.2 & 7915 & -114.705999 & 5036 & 4604 & 4521 \\
\hline 2.5719 & 1.4 & -114.848013 & -114.856734 & -114.855693 & -114.855209 & -114.854805 \\
\hline 2.5719 & 1.6 & -114.907878 & -114.917396 & -114.916253 & -114.915708 & -114.915561 \\
\hline 2.5719 & 1.7325 & 3212 & -114. & -11 & -114 & 1650 \\
\hline 2.5719 & 1.8 & 26897 & 37475 & 6182 & 5562 & 35799 \\
\hline 2.5719 & 2.0 & -114.929484 & -114.941552 & -114.939998 & -114.939283 & -114.940253 \\
\hline 2.5719 & 2.25 & -114.928381 & -114.942971 & -114.940785 & -114.939899 & -114.941910 \\
\hline 2.5719 & 2.5 & -114 & -114 & -114 & -114 & 47004 \\
\hline 2.5719 & 2.75 & -114.936836 & -114.954680 & 50853 & 19763 & 953650 \\
\hline 2.5719 & 3.0 & -114.942712 & -114.960590 & -114.956223 & -114.955220 & -114.959383 \\
\hline 2.5719 & 3.5 & -114.950775 & -114.968139 & -114.962895 & -114.962050 & -114.966366 \\
\hline 2.5719 & 4.0 & 4307 & -114 & -114 & -114 & 68990 \\
\hline 2.5719 & 5.0 & -114.955251 & -114.973267 & -114.965213 & -114.964342 & -114.969097 \\
\hline 2.5719 & 6.0 & -114.954445 & -114.973654 & -114.963922 & -114.962991 & -114.968097 \\
\hline 2.5719 & 8.0 & -114.953639 & -114.974179 & -114.962754 & -114.961844 & -114.967323 \\
\hline 2.6 & 1.2 & -114.701515 & -114.709604 & -114 & -114 & -114.707554 \\
\hline 2.6 & 1.4 & -114.851375 & -114.860100 & -114.859053 & -114.858569 & -114.858156 \\
\hline 2.6 & 1.6 & -114.910915 & -114.920434 & -114.919284 & -114.918739 & -114.918582 \\
\hline 2.6 & 1.7325 & -114.925973 & -114.936151 & -114.934911 & -114.934319 & -114.934388 \\
\hline 2.6 & 1.8 & -114.929496 & -114.940069 & -114.938770 & -114.938151 & -114.938370 \\
\hline 2.6 & 2.0 & -114.931508 & -114.943562 & -114.942006 & -114.941292 & -114.942240 \\
\hline 2.6 & 2.25 & -114.929552 & -114.944149 & -114.941963 & -114.941078 & -114.943071 \\
\hline 2.6 & 2.5 & -114.931528 & -114.948498 & -114.945396 & -114.944326 & -114.947531 \\
\hline 2.6 & 2.75 & -114.936857 & -114.954890 & -114.951003 & -114.949895 & -114.953830 \\
\hline 2.6 & 3.0 & -114.942521 & -114.960616 & -114.956170 & -114.955148 & -114.959372 \\
\hline 2.6 & 3.5 & -114.950446 & -114.968002 & -114.962670 & -114.961815 & -114.966197 \\
\hline 2.6 & 4.0 & -114.953982 & -114.971347 & -114.965126 & -114.964316 & -114.968794 \\
\hline 2.6 & 5.0 & -114.955013 & -114.973108 & -114.965006 & -114.964142 & -114.968946 \\
\hline 2.6 & 6.0 & -114.954261 & -114.973492 & -114.963753 & -114.962835 & -114.967984 \\
\hline 2.6 & 8.0 & -114.953481 & -114.973994 & -114.962606 & -114.961713 & -114.967230 \\
\hline 2.7 & 1.2 & -114.712202 & -114.720303 & -114.719316 & -114.718885 & -114.718221 \\
\hline
\end{tabular}


Table S3. (continued)

\begin{tabular}{|c|c|c|c|c|c|c|}
\hline$R_{\mathrm{Be}-\mathrm{F}}$ & $R_{\mathrm{H}-\mathrm{F}}$ & CCSD & $\operatorname{CCSD}(\mathrm{T})$ & R-CCSD $(\mathrm{T})$ & CR-CCSD(T) & $\operatorname{MRCI}(\mathrm{Q})$ \\
\hline 2.7 & 1.4 & -114.861262 & -114.869996 & -114.868928 & -114.868445 & -114.868002 \\
\hline 2.7 & 1.6 & -114.919709 & -114.929226 & -114.928056 & -114.927512 & -114.927320 \\
\hline 2.7 & 1.7325 & -114.933844 & -114.944005 & -114.942747 & -114.942156 & -114.942178 \\
\hline 2.7 & 1.8 & -114.936823 & -114.947368 & -114.946052 & -114.945435 & -114.945596 \\
\hline 2.7 & 2.0 & -114.936886 & -114.948872 & -114.947313 & -114.946604 & -114.947454 \\
\hline 2.7 & 2.25 & -114.931940 & -114.946511 & -114.944336 & -114.943460 & -114.945377 \\
\hline 2.7 & 2.5 & -114.931249 & -114.948524 & -114.945353 & -114.944264 & -114.947483 \\
\hline 2.7 & 2.75 & -114.934979 & -114.953666 & -114.949575 & -114.948404 & -114.952492 \\
\hline 2.7 & 3.0 & -114.939851 & -114.958727 & -114.953997 & -114.952905 & -114.957341 \\
\hline 2.7 & 3.5 & -114.947256 & -114.965513 & -114.959863 & -114.958974 & -114.963583 \\
\hline 2.7 & 4.0 & -114.950792 & -114.968709 & -114.962194 & -114.961378 & -114.966068 \\
\hline 2.7 & 5.0 & -114.952097 & -114.970489 & -114.962214 & -114.961375 & -114.966353 \\
\hline 2.7 & 6.0 & -114.951518 & -114.970858 & -114.961084 & -114.960210 & -114.965508 \\
\hline 2.7 & 8.0 & -114.950827 & -114.971295 & -114.960010 & -114.959170 & -114.964818 \\
\hline 2.9 & 1.2 & -114.726479 & -114.734576 & -114.733564 & -114.733135 & -114.732423 \\
\hline 2.9 & 1.4 & -114.874129 & -114.882849 & -114.881755 & -114.881273 & -114.880779 \\
\hline 2.9 & 1.6 & -114.930675 & -114.940154 & -114.938956 & -114.938415 & -114.938162 \\
\hline 2.9 & 1.7325 & -114.943212 & -114.953302 & -114.952018 & -114.9 & -114 \\
\hline 2.9 & 1.8 & -114.945247 & -114.955695 & -114.954359 & -114.953748 & -114.953802 \\
\hline 2.9 & 2.0 & -114.941876 & -114.953657 & -114.952105 & -114.951409 & -114.951999 \\
\hline 2.9 & 2.25 & -114.931286 & -114.945581 & -114.943483 & -114.942639 & -114.944387 \\
\hline 2.9 & 2.5 & -114.924892 & -114. & -114 . & 38161 & -114.941244 \\
\hline 2.9 & 2.75 & -114. & -114.944757 & -114.940337 & -114.939062 & -114.943363 \\
\hline 2.9 & 3.0 & -114.927971 & -114.948449 & -114.943152 & -114.941903 & -114.946719 \\
\hline 2.9 & 3.5 & -114.934198 & -114.953972 & -114.947655 & -114.946672 & -114.951717 \\
\hline 2.9 & 4.0 & -114.937660 & -114.956765 & -114.949648 & -114.948808 & -114.953906 \\
\hline 2.9 & 5.0 & -114.939387 & -114.958461 & -114.949819 & -114.949026 & -114.954335 \\
\hline 2.9 & 6.0 & -114.939082 & -114.958791 & -114.948880 & -114.948084 & -114.953665 \\
\hline 2.9 & 8.0 & -114.938535 & -114.959149 & -114.947936 & -114.947184 & -114.953079 \\
\hline 3.1 & 1.2 & -114.735257 & -114 & -114.742288 & -114.741861 & -114.741110 \\
\hline 3.1 & 1.4 & -114.881722 & -114.890386 & -114.889280 & -114.888803 & -114.888265 \\
\hline 3.1 & 1.6 & -114.936698 & -114.946095 & -114.944886 & -114.944350 & -114.944045 \\
\hline 3.1 & 1.7325 & -114.947931 & -114.957904 & -114.956612 & -114.956033 & -114.955902 \\
\hline 3.1 & 1.8 & -114.949198 & -114.959504 & -114.958162 & -114.957560 & -114.957529 \\
\hline 3.1 & 2.0 & -114.943015 & -114.954529 & -114.952997 & -114.952317 & -114.952677 \\
\hline 3.1 & 2.25 & -114.927472 & -114.941271 & -114.939290 & -114.938484 & -114.940097 \\
\hline 3.1 & 2.5 & -114.915128 & -114.932352 & -114.929356 & -114.928334 & -114.931021 \\
\hline 3.1 & 2.75 & -114.910603 & -114.931166 & -114.926614 & -114.925301 & -114.929630 \\
\hline 3.1 & 3.0 & -114.911343 & -114.933398 & -114.927576 & -114.926167 & -114.931288 \\
\hline 3.1 & 3.5 & -114.916152 & -114.937654 & -114.930603 & -114.929480 & -114.934930 \\
\hline 3.1 & 4.0 & -114.919462 & -114.939931 & -114.932157 & -114.931265 & -114.936751 \\
\hline 3.1 & 5.0 & -114.921482 & -114.941398 & -114.932335 & -114.931573 & -114.937198 \\
\hline 3.1 & 6.0 & -114.921378 & -114.941673 & -114.931523 & -114.930786 & -114.936636 \\
\hline 3.1 & 8.0 & -114.920938 & -114.941995 & -114.930685 & -114.929997 & -114.936122 \\
\hline 3.3 & 1.2 & -114.741309 & -114.749265 & -114.748251 & -114.747830 & -114.747318 \\
\hline 3.3 & 1.4 & -114.886784 & -114.895342 & -114.894242 & -114.893770 & -114.893213 \\
\hline 3.3 & 1.6 & -114.940475 & -114.949741 & -114.948536 & -114.948008 & -114.947655 \\
\hline 3.3 & 1.7325 & -114.950656 & -114.960466 & -114.959181 & -114.958612 & -114.958428 \\
\hline 3.3 & 1.8 & -114.951310 & -114.961429 & -114.960097 & -114.959505 & -114.959413 \\
\hline
\end{tabular}


Table S3. (continued)

\begin{tabular}{|c|c|c|c|c|c|c|}
\hline$R_{\mathrm{Be}-\mathrm{F}}$ & $R_{\mathrm{H}-\mathrm{F}}$ & CCSD & $\operatorname{CCSD}(\mathrm{T})$ & $\mathrm{R}-\mathrm{CCSD}(\mathrm{T})$ & CR-CCSD $(\mathrm{T})$ & $\operatorname{MRCI}(\mathrm{Q})$ \\
\hline 3.3 & 2.0 & -114.942897 & -114.954106 & -114.952603 & -114.951941 & -114.952161 \\
\hline 3.3 & 2.25 & -114.923304 & -114.936503 & -114.934641 & -114.933870 & -114.934985 \\
\hline 3.3 & 2.5 & -114.905292 & -114.921701 & -114.919017 & -114.918082 & -114.920207 \\
\hline 3.3 & 2.75 & -114.895312 & -114.915857 & -114.911497 & -114.910250 & -114.914298 \\
\hline 3.3 & 3.0 & -114.892946 & -114.916315 & -114.910124 & -114.908610 & -114.913917 \\
\hline 3.3 & 3.5 & -114.895948 & -114.919454 & -114.911560 & -114.910250 & -114.916069 \\
\hline 3.3 & 4.0 & -114.899049 & -114.921142 & -114.912607 & -114.911623 & -114.917468 \\
\hline 3.3 & 5.0 & -114.901280 & -114.922242 & -114.912669 & -114.911917 & -114.917844 \\
\hline 3.3 & 6.0 & -114.901325 & -114.922433 & -114.911931 & -114.911233 & -114.917337 \\
\hline 3.3 & 8.0 & -114.900968 & -114.922736 & -114.911176 & -114.910530 & -114.916869 \\
\hline 3.5 & 1.2 & -114.746030 & -114.753843 & -114.752848 & -114.752433 & -114.751817 \\
\hline 3.5 & 1.4 & -114.890687 & -114.899089 & -114.898006 & -114.897543 & -114.897763 \\
\hline 3.5 & 1.6 & -114.943334 & -114.952419 & -114.951233 & -114.950715 & -114.950323 \\
\hline 3.5 & 1.7325 & -114.952676 & -114.962277 & -114.961012 & -114.960455 & -114.960226 \\
\hline 3.5 & 1.8 & -114.952848 & -114.962736 & -114.961427 & -114.960849 & -114.960707 \\
\hline 3.5 & 2.0 & -114.942712 & -114.953589 & -114.952123 & -114.951479 & -114.951616 \\
\hline 3.5 & 2.25 & -114.920012 & -114.932596 & -114.930837 & -114.930098 & -114.930719 \\
\hline 3.5 & 2.5 & -114.897209 & -114.912534 & -114.910174 & -114.909314 & -114.911182 \\
\hline 3.5 & 2.75 & -114.881296 & -114.900884 & -114.897063 & -114.895969 & -114.899247 \\
\hline 3.5 & 3.0 & -114.874655 & -114.898607 & -114.892433 & -114.890944 & -114.896239 \\
\hline 3.5 & 3.5 & -114.875135 & -114.900945 & -114.892081 & -114.890544 & -114.896692 \\
\hline 3.5 & 4.0 & -114.877982 & -114.902057 & -114.892590 & -114.891463 & -114.897637 \\
\hline 3.5 & 5.0 & -114.880386 & -114.902637 & -114.892433 & -114.891667 & -114.897877 \\
\hline 3.5 & 6.0 & -114.880547 & -114.902701 & -114.891721 & -114.891043 & -114.897387 \\
\hline 3.5 & 8.0 & -114.880255 & -114.902980 & -114.891029 & -114.890406 & -114.896944 \\
\hline 3.7 & 1.2 & -114.750061 & -114.757688 & -114.756720 & -114.756314 & -114.756204 \\
\hline 3.7 & 1.4 & -114.894047 & -114.902249 & -114.901194 & -114.900741 & -114.900795 \\
\hline 3.7 & 1.6 & -114.945854 & -114.954716 & -114.953559 & -114.953053 & -114.953339 \\
\hline 3.7 & 1.7325 & -114.954535 & -114.963887 & -114.962653 & -114.962110 & -114.961840 \\
\hline 3.7 & 1.8 & -114.954330 & -114.963952 & -114.962676 & -114.962113 & -114.961928 \\
\hline 3.7 & 2.0 & -114.942886 & -114.953412 & -114.951989 & -114.951366 & -114.951447 \\
\hline 3.7 & 2.25 & -114.917908 & -114.929912 & -114.928238 & -114.927529 & -114.927980 \\
\hline 3.7 & 2.5 & -114.891506 & -114.905754 & -114.903643 & -114.902836 & -114.904273 \\
\hline 3.7 & 2.75 & -114.870122 & -114.888082 & -114.884939 & -114.883992 & -114.885950 \\
\hline 3.7 & 3.0 & -114.857972 & -114.881205 & -114.875675 & -114.874379 & -114.879249 \\
\hline 3.7 & 3.5 & -114.854583 & -114.882882 & -114.872977 & -114.871220 & -114.877668 \\
\hline 3.7 & 4.0 & -114.857105 & -114.883634 & -114.872986 & -114.871660 & -114.878126 \\
\hline 3.7 & 5.0 & -114.859688 & -114.883529 & -114.872530 & -114.871719 & -114.878190 \\
\hline 3.7 & 6.0 & -114.859952 & -114.883400 & -114.871802 & -114.871124 & -114.877689 \\
\hline 3.7 & 8.0 & -114.859714 & -114.883634 & -114.871151 & -114.870536 & -114.877255 \\
\hline 3.9 & 1.2 & -114.753642 & -114.761056 & -114.760120 & -114.759724 & -114.759461 \\
\hline 3.9 & 1.4 & -114.897087 & -114.905060 & -114.904038 & -114.903596 & -114.903494 \\
\hline 3.9 & 1.6 & -114.948227 & -114.956835 & -114.955715 & -114.955222 & -114.955330 \\
\hline 3.9 & 1.7325 & -114.956389 & -114.965466 & -114.964270 & -114.963742 & -114.964013 \\
\hline 3.9 & 1.8 & -114.955895 & -114.965227 & -114.963990 & -114.963444 & -114.963219 \\
\hline 3.9 & 2.0 & -114.943466 & -114.953635 & -114.952259 & -114.951656 & -114.951692 \\
\hline 3.9 & 2.25 & -114.916859 & -114.928333 & -114.926732 & -114.926052 & -114.926431 \\
\hline 3.9 & 2.5 & -114.887978 & -114.901296 & -114.899354 & -114.898587 & -114.899350 \\
\hline 3.9 & 2.75 & -114.862435 & -114.878697 & -114.876086 & -114.875222 & -114.876133 \\
\hline
\end{tabular}


Table S3. (continued)

\begin{tabular}{|c|c|c|c|c|c|c|}
\hline$R_{\mathrm{Be}-\mathrm{F}}$ & $R_{\mathrm{H}-\mathrm{F}}$ & CCSD & $\operatorname{CCSD}(\mathrm{T})$ & $\mathrm{R}-\mathrm{CCSD}(\mathrm{T})$ & CR-CCSD $(\mathrm{T})$ & $\operatorname{MRCI}(\mathrm{Q})$ \\
\hline 3.9 & 3.0 & -114.844390 & -114.865550 & -114.861172 & -114.860129 & -114.863694 \\
\hline 3.9 & 3.5 & -114.834831 & -114.865359 & -114.854618 & -114.852746 & -114.859479 \\
\hline 3.9 & 4.0 & -114.836860 & -114.866428 & -114.854269 & -114.852693 & -114.859418 \\
\hline 3.9 & 5.0 & -114.839670 & -114.865483 & -114.853468 & -114.852578 & -114.859284 \\
\hline 3.9 & 6.0 & -114.840043 & -114.865069 & -114.852685 & -114.851987 & -114.858756 \\
\hline 3.9 & 8.0 & -114.839854 & -114.865217 & -114.852054 & -114.851436 & -114.858318 \\
\hline 4.1 & 1.2 & -114.756836 & -114.764025 & -114.763121 & -114.762736 & -114.760975 \\
\hline 4.1 & 1.4 & -114.899848 & -114.907579 & -114.906592 & -114.906163 & -114.905914 \\
\hline 4.1 & 1.6 & -114.950463 & -114.958807 & -114.957723 & -114.957245 & -114.957201 \\
\hline 4.1 & 1.7325 & -114.958224 & -114.967016 & -114.965860 & -114.965348 & -114.965454 \\
\hline 4.1 & 1.8 & -114.957508 & -114.966542 & -114.965346 & -114.964817 & -114.965004 \\
\hline 4.1 & 2.0 & -114.944341 & -114.954159 & -114.952830 & -114.952248 & -114.952238 \\
\hline 4.1 & 2.25 & -114.916580 & -114.927576 & -114.926039 & -114.925387 & -114.925725 \\
\hline 4.1 & 2.5 & -114.886065 & -114.898625 & -114.896798 & -114.896067 & -114.896753 \\
\hline 4.1 & 2.75 & -114.857826 & -114.872705 & -114.870405 & -114.869584 & -114 \\
\hline 4.1 & 3.0 & -114.835000 & -114.853691 & -114.850349 & -114.849440 & -114.850392 \\
\hline 4.1 & 3.5 & -114.816401 & -114.847815 & -114.837135 & -114.835394 & -114.842402 \\
\hline 4.1 & 4.0 & -114.817456 & -114.850675 & -114.836654 & -114.834811 & -114 \\
\hline 4.1 & 5.0 & -114.820582 & -114.848865 & -114.835532 & -114.834526 & 41437 \\
\hline 4.1 & 6.0 & -114.821087 & -114.848031 & -114.834656 & -114.833920 & -114.840876 \\
\hline 4.1 & 8.0 & -114.820952 & -114.848037 & -114.834026 & -114.833394 & -114.840424 \\
\hline 4.5 & 1.2 & -114.762048 & -114.7 & -114.767961 & -114.767598 & -114 \\
\hline 4.5 & 1.4 & -114.904451 & -114. & -114.910796 & -114.910391 & -114.908425 \\
\hline 4.5 & 1.6 & -114.954340 & -114.962180 & -114.961166 & -114.960716 & -114.958582 \\
\hline 4.5 & 1.7325 & -114.961555 & -114.969809 & -114.968727 & -114.968246 & -114.968092 \\
\hline 4.5 & 1.8 & -114.960541 & -114.969017 & -114.967898 & -114.967402 & -114 \\
\hline 4.5 & 2.0 & -114.946410 & -114.955594 & -114.954351 & -114.953808 & -114.953968 \\
\hline 4.5 & 2.25 & -114.917244 & -114.927445 & -114.926014 & -114.925411 & -114.925871 \\
\hline 4.5 & 2.5 & -114.884964 & -114.896414 & -114.894737 & -114.894066 & -114.894780 \\
\hline 4.5 & 2.75 & -114.854190 & -114.867291 & -114.865264 & -114.864511 & -114.865313 \\
\hline 4.5 & 3.0 & -114.826888 & -114.842347 & -114.839787 & -114.838929 & -114.839340 \\
\hline 4.5 & 3.5 & -114.788314 & -114.813428 & -114.807480 & -114.806485 & -114.812197 \\
\hline 4.5 & 4.0 & -114.781546 & -114.821878 & -114.804589 & -114.802571 & -114.810144 \\
\hline 4.5 & 5.0 & -114.785500 & -114.820984 & -114.803640 & -114.802270 & -114.809436 \\
\hline 4.5 & 6.0 & -114.786447 & -114.818665 & -114.802452 & -114.801573 & -114.808820 \\
\hline 4.5 & 8.0 & -114.786470 & -114.818114 & -114.801754 & -114.801068 & -114.808332 \\
\hline 4.7 & 1.2 & -114.764067 & -114.770637 & -114.769818 & -114.769464 & -114.767682 \\
\hline 4.7 & 1.4 & -114.906263 & -114.913329 & -114.912433 & -114.912039 & -114.910073 \\
\hline 4.7 & 1.6 & -114.955907 & -114.963529 & -114.962544 & -114.962107 & -114.959975 \\
\hline 4.7 & 1.7325 & -114.962942 & -114.970963 & -114.969912 & -114.969446 & -114.969186 \\
\hline 4.7 & 1.8 & -114.961830 & -114.970066 & -114.968979 & -114.968497 & -114.968314 \\
\hline 4.7 & 2.0 & -114.947391 & -114.956306 & -114.955099 & -114.954573 & -114.954627 \\
\hline 4.7 & 2.25 & -114.917801 & -114.927683 & -114.926295 & -114.925712 & -114.926074 \\
\hline 4.7 & 2.5 & -114.885051 & -114.896097 & -114.894472 & -114.893826 & -114.894474 \\
\hline 4.7 & 2.75 & -114.853727 & -114.866266 & -114.864310 & -114.863585 & -114.864433 \\
\hline 4.7 & 3.0 & -114.825676 & -114.840265 & -114.837816 & -114.836983 & -114.837797 \\
\hline 4.7 & 3.5 & -114.782177 & -114.804049 & -114.799574 & -114.798489 & -114.798553 \\
\hline 4.7 & 4.0 & -114.765554 & -114.805460 & -114.789700 & -114.788078 & -114.796238 \\
\hline 4.7 & 5.0 & -114.769456 & -114.810248 & -114.789873 & -114.788248 & -114.795390 \\
\hline
\end{tabular}


Table S3. (continued)

\begin{tabular}{|c|c|c|c|c|c|c|}
\hline$R_{\mathrm{Be}-\mathrm{F}}$ & $R_{\mathrm{H}-\mathrm{F}}$ & CCSD & $\operatorname{CCSD}(\mathrm{T})$ & $\mathrm{R}-\mathrm{CCSD}(\mathrm{T})$ & CR-CCSD(T) & MRCI(Q) \\
\hline 4.7 & 6.0 & -114.770775 & -114.806732 & -114.788474 & -114.787473 & -114.794773 \\
\hline 4.7 & 8.0 & -114.770928 & -114.805683 & -114.787692 & -114.786955 & -114.794273 \\
\hline 5.0 & 1.2 & -114.766430 & -114.772763 & -114.771975 & -114.771633 & -114.769852 \\
\hline 5.0 & 1.4 & -114.908400 & -114.915211 & -114.914348 & -114.913968 & -114.912006 \\
\hline 5.0 & 1.6 & -114.957779 & -114.965124 & -114.964176 & -114.963754 & -114.961631 \\
\hline 5.0 & 1.7325 & -114.964618 & -114.972348 & -114.971335 & -114.970886 & -114.968679 \\
\hline 5.0 & 1.8 & -114.963402 & -114.971337 & -114.970289 & -114.969826 & -114.969511 \\
\hline 5.0 & 2.0 & -114.948636 & -114.957218 & -114.956055 & -114.955550 & -114.955473 \\
\hline 5.0 & 2.25 & -114.918614 & -114.928108 & -114.926771 & -114.926213 & -114.926451 \\
\hline 5.0 & 2.5 & -114.885418 & -114.895994 & -114.894430 & -114.893814 & -114.894372 \\
\hline 5.0 & 2.75 & -114.853641 & -114.865574 & -114.863694 & -114.863004 & -114.863858 \\
\hline 5.0 & 3.0 & -114.825117 & -114.838857 & -114.836506 & -114.835708 & -114.836789 \\
\hline 5.0 & 3.5 & -114.779727 & -114.799686 & -114.795394 & -114.794175 & -114.795564 \\
\hline 5.0 & 4.0 & -114.749851 & -114.781383 & -114.772754 & -114.771162 & -114.771304 \\
\hline 5.0 & 5.0 & -114.747072 & -114.798861 & -114.772275 & -114.770177 & -114.776862 \\
\hline 5.0 & 6.0 & -114.749222 & -114.793308 & -114.770636 & -114.769281 & -114.776314 \\
\hline 5.0 & 8.0 & -114.749686 & -114.791013 & -114.769603 & -114.768680 & -114.775828 \\
\hline 5.2 & 1.2 & -114.767620 & -114.773825 & -114.773052 & -114.772717 & -114.770939 \\
\hline 5.2 & 1.4 & -114.909480 & -114.916153 & -114.915308 & -114.914935 & -114.912978 \\
\hline 5.2 & 1.6 & -114.958732 & -114.965928 & -114.964999 & -114.964586 & -114.962470 \\
\hline 5.2 & 1.7325 & -114.965478 & -114.973050 & -114.972058 & -114.971618 & -114.969420 \\
\hline 5.2 & 1.8 & -114.964213 & -114.971984 & -114.970958 & -114.970504 & -114.968275 \\
\hline 5.2 & 2.0 & -114.949293 & -114.957695 & -114.956556 & -114.956062 & -114.953786 \\
\hline 5.2 & 2.25 & -114.919073 & -114.928361 & -114.927051 & -114.926506 & -114.924287 \\
\hline 5.2 & 2.5 & -114.885685 & -114.896020 & -114.894487 & -114.893887 & -114.894388 \\
\hline 5.2 & 2.75 & -114.853739 & -114.865377 & -114.863534 & -114.862861 & -114.863690 \\
\hline 5.2 & 3.0 & -114.825084 & -114.838440 & -114.836136 & -114.835356 & -114.836309 \\
\hline 5.2 & 3.5 & -114.779596 & -114.798845 & -114.794571 & -114.793348 & -114.795099 \\
\hline 5.2 & 4.0 & -114.748527 & -114.779674 & -114.770515 & -114.768254 & -114.770996 \\
\hline 5.2 & 5.0 & -114.733354 & -114.794346 & -114.763084 & -114.760542 & -114.766323 \\
\hline 5.2 & 6.0 & -114.736193 & -114.788734 & -114.761529 & -114.759646 & -114.765917 \\
\hline 5.2 & 8.0 & -114.736964 & -114.785052 & -114.760175 & -114.758903 & -114.765496 \\
\hline 5.5 & 1.2 & -114.768939 & -114.774989 & -114.774236 & -114.773908 & -114.772134 \\
\hline 5.5 & 1.4 & -114.910679 & -114.917185 & -114.916361 & -114.915997 & -114.914047 \\
\hline 5.5 & 1.6 & -114.959792 & -114.966807 & -114.965900 & -114.965498 & -114.963392 \\
\hline 5.5 & 1.7325 & -114.966436 & -114.973816 & -114.972848 & -114.972420 & -114.971680 \\
\hline 5.5 & 1.8 & -114.965117 & -114.972691 & -114.971690 & -114.971248 & -114.970572 \\
\hline 5.5 & 2.0 & -114.950031 & -114.958217 & -114.957105 & -114.956625 & -114.954371 \\
\hline 5.5 & 2.25 & -114.919602 & -114.928645 & -114.927366 & -114.926838 & -114.924653 \\
\hline 5.5 & 2.5 & -114.886023 & -114.896074 & -114.894578 & -114.893996 & -114.894421 \\
\hline 5.5 & 2.75 & -114.853923 & -114.865227 & -114.863427 & -114.862774 & -114.863246 \\
\hline 5.5 & 3.0 & -114.825182 & -114.838126 & -114.835874 & -114.835116 & -114.835945 \\
\hline 5.5 & 3.5 & -114.779875 & -114.798340 & -114.794172 & -114.792967 & -114.794952 \\
\hline 5.5 & 4.0 & -114.749609 & -114.780373 & -114.770471 & -114.768003 & -114.771317 \\
\hline 5.5 & 5.0 & -114.718761 & -114.792016 & -114.757926 & -114.752173 & -114.754938 \\
\hline 5.5 & 6.0 & -114.719691 & -114.795699 & -114.755559 & -114.751333 & -114.757844 \\
\hline 5.5 & 8.0 & -114.720969 & -114.787942 & -114.753029 & -114.750041 & -114.757833 \\
\hline 6.0 & 1.2 & -114.770231 & -114.776098 & -114.775367 & -114.775049 & -114.773282 \\
\hline 6.0 & 1.4 & -114.911845 & -114.918154 & -114.917354 & -114.917000 & -114.915061 \\
\hline
\end{tabular}


Table S3. (continued)

\begin{tabular}{lcccccc}
\hline$R_{\mathrm{Be}-\mathrm{F}}$ & $R_{\mathrm{H}-\mathrm{F}}$ & $\mathrm{CCSD}$ & $\mathrm{CCSD}(\mathrm{T})$ & $\mathrm{R}-\mathrm{CCSD}(\mathrm{T})$ & $\mathrm{CR}-\mathrm{CCSD}(\mathrm{T})$ & $\mathrm{MRCI}(\mathrm{Q})$ \\
\hline 6.0 & 1.6 & -114.960812 & -114.967614 & -114.966734 & -114.966344 & -114.964253 \\
6.0 & 1.7325 & -114.967353 & -114.974507 & -114.973568 & -114.973153 & -114.972335 \\
6.0 & 1.8 & -114.965978 & -114.973320 & -114.972348 & -114.971920 & -114.971157 \\
6.0 & 2.0 & -114.950726 & -114.958658 & -114.957579 & -114.957115 & -114.956537 \\
6.0 & 2.25 & -114.920094 & -114.928851 & -114.927610 & -114.927100 & -114.926795 \\
6.0 & 2.5 & -114.886340 & -114.896069 & -114.894615 & -114.894053 & -114.894048 \\
6.0 & 2.75 & -114.854122 & -114.865057 & -114.863306 & -114.862676 & -114.863004 \\
6.0 & 3.0 & -114.825348 & -114.837863 & -114.835669 & -114.834933 & -114.835683 \\
6.0 & 3.5 & -114.780387 & -114.798064 & -114.794067 & -114.792888 & -114.795004 \\
6.0 & 4.0 & -114.751241 & -114.779561 & -114.770579 & -114.768248 & -114.771927 \\
6.0 & 5.0 & -114.724002 & -114.816921 & -114.765901 & -114.754797 & -114.756184 \\
6.0 & 6.0 & -114.713298 & -114.871502 & -114.773720 & -114.755544 & -114.753512 \\
6.0 & 8.0 & -114.711166 & -114.860289 & -114.768805 & -114.754732 & -114.755243 \\
8.0 & 1.2 & -114.770926 & -114.776525 & -114.775825 & -114.775521 & -114.773782 \\
8.0 & 1.4 & -114.912361 & -114.918379 & -114.917614 & -114.917276 & -114.916327 \\
8.0 & 1.6 & -114.961136 & -114.967620 & -114.966779 & -114.966407 & -114.965502 \\
8.0 & 1.7325 & -114.967544 & -114.974362 & -114.973465 & -114.973070 & -114.972231 \\
8.0 & 1.8 & -114.966102 & -114.973097 & -114.972169 & -114.971762 & -114.970965 \\
8.0 & 2.0 & -114.950654 & -114.958207 & -114.957177 & -114.956737 & -114.956090 \\
8.0 & 2.25 & -114.919808 & -114.928142 & -114.926957 & -114.926474 & -114.926063 \\
8.0 & 2.5 & -114.885900 & -114.895159 & -114.893769 & -114.893238 & -114.893105 \\
8.0 & 2.75 & -114.853620 & -114.864037 & -114.862357 & -114.861759 & -114.861961 \\
8.0 & 3.0 & -114.824903 & -114.836846 & -114.834734 & -114.834032 & -114.834687 \\
8.0 & 3.5 & -114.780493 & -114.797320 & -114.793511 & -114.792375 & -114.794520 \\
8.0 & 4.0 & -114.752490 & -114.777868 & -114.770251 & -114.768146 & -114.772136 \\
8.0 & 5.0 & -114.728423 & -114.782433 & -114.757803 & -114.751755 & -114.757093 \\
8.0 & 6.0 & -114.721781 & -114.823308 & -114.764464 & -114.751698 & -114.754553 \\
8.0 & 8.0 & -114.718611 & -114.906140 & -114.780009 & -114.756643 & -114.753938 \\
\hline & & & & & &
\end{tabular}

Table S4. The CCSD, CCSD(T), R-CCSD(T), CR-CCSD(T), and MRCI(Q) energies, in hartree, of the BeFH system, as described by the cc-pVTZ basis set, for the Be-F-H angle $\theta=80^{\circ}$. The Be $-\mathrm{F}$ and $\mathrm{H}-\mathrm{F}$ bond distances, $R_{\mathrm{Be}-\mathrm{F}}$ and $R_{\mathrm{H}-\mathrm{F}}$, respectively, are in bohr.

\begin{tabular}{lcccccc}
\hline$R_{\mathrm{Be}-\mathrm{F}}$ & $R_{\mathrm{H}-\mathrm{F}}$ & $\mathrm{CCSD}$ & $\mathrm{CCSD}(\mathrm{T})$ & $\mathrm{R}-\mathrm{CCSD}(\mathrm{T})$ & $\mathrm{CR}-\mathrm{CCSD}(\mathrm{T})$ & $\mathrm{MRCI}(\mathrm{Q})$ \\
\hline 1.8 & 1.2 & -114.281285 & -114.289074 & -114.288347 & -114.287927 & -114.287562 \\
1.8 & 1.4 & -114.444818 & -114.453258 & -114.452468 & -114.451997 & -114.451846 \\
1.8 & 1.6 & -114.524001 & -114.533241 & -114.532364 & -114.531833 & -114.532003 \\
1.8 & 1.7325 & -114.554972 & -114.564812 & -114.563858 & -114.563283 & -114.563731 \\
1.8 & 1.8 & -114.567283 & -114.577434 & -114.576436 & -114.575838 & -114.576440 \\
1.8 & 2.0 & -114.596406 & -114.607385 & -114.606241 & -114.605584 & -114.606626 \\
1.8 & 2.25 & -114.624723 & -114.636258 & -114.634946 & -114.634255 & -114.635691 \\
1.8 & 2.5 & -114.646923 & -114.658476 & -114.657040 & -114.656362 & -114.657988 \\
1.8 & 2.75 & -114.663242 & -114.674563 & -114.673030 & -114.672385 & -114.674081 \\
1.8 & 3.0 & -114.674212 & -114.685277 & -114.683648 & -114.683031 & -114.684758 \\
1.8 & 3.5 & -114.683948 & -114.694778 & -114.692873 & -114.692288 & -114.694111 \\
\hline
\end{tabular}


Table S4. (continued)

\begin{tabular}{|c|c|c|c|c|c|c|}
\hline$R_{\mathrm{Be}-\mathrm{F}}$ & $R_{\mathrm{H}-\mathrm{F}}$ & CCSD & $\operatorname{CCSD}(\mathrm{T})$ & R-CCSD $(\mathrm{T})$ & CR-CCSD(T) & MRCI(Q) \\
\hline 1.8 & 4.0 & -114.683820 & -114.694919 & -114.692524 & -114.691936 & -114.693986 \\
\hline 1.8 & 5.0 & -114.674806 & -114.687969 & -114.683630 & -114.682917 & -114.685725 \\
\hline 1.8 & 6.0 & -114.667912 & -114.684683 & -114.677169 & -114.676203 & -114.679712 \\
\hline 1.8 & 8.0 & -114.663747 & -114.686593 & -114.673568 & -114.672226 & -114.676357 \\
\hline 1.9 & 1.2 & -114.395693 & -114.403566 & -114.402802 & -114.402377 & -114.401991 \\
\hline 1.9 & 1.4 & -114.556910 & -114.565447 & -114.564617 & -114.564140 & -114.563975 \\
\hline 1.9 & 1.6 & -114.633056 & -114.642416 & -114.641495 & -114.640956 & -114.641120 \\
\hline 1.9 & 1.7325 & -114.661787 & -114.671780 & -114.670778 & -114.670193 & -114.670651 \\
\hline 1.9 & 1.8 & -114.672944 & -114.683274 & -114.682224 & -114.681615 & -114.682240 \\
\hline 1.9 & 2.0 & -114.698873 & -114.710142 & -114.708928 & -114.708251 & -114.709367 \\
\hline 1.9 & 2.25 & -114.724184 & -114.736166 & -114.734753 & -114.734030 & -114.735605 \\
\hline 1.9 & 2.5 & -114.744576 & -114.756664 & -114.755099 & -114.754386 & -114.756190 \\
\hline 1.9 & 2.75 & -114.759930 & -114.771805 & -114.770125 & -114.769445 & -114.771333 \\
\hline 1.9 & 3.0 & -114.770450 & -114.782054 & -114.780264 & -114.779617 & -114.781537 \\
\hline 1.9 & 3.5 & -114.780120 & -114.791437 & -114.789347 & -114.788740 & -114.790747 \\
\hline 1.9 & 4.0 & -114.780427 & -114.791968 & -114.789364 & -114.788757 & -114.790987 \\
\hline 1.9 & 5.0 & -114.772475 & -114.785967 & -114.781398 & -114.780674 & -114.783655 \\
\hline 1.9 & 6.0 & -114.766229 & -114.783043 & -114.775441 & -114.774485 & -114.778162 \\
\hline 1.9 & 8.0 & -114.762420 & -114.784602 & -114.772013 & -114.770737 & -114.775051 \\
\hline 2.0 & 1.2 & -114.481209 & -114.489159 & -114.488358 & -114.487930 & -114.487525 \\
\hline 2.0 & 1.4 & -114.640368 & -114.648992 & -114.648123 & -114.647641 & -114.647457 \\
\hline 2.0 & 1.6 & -114.713755 & -114.723222 & -114.722258 & -114.721712 & -114.721871 \\
\hline 2.0 & .7325 & -114.740401 & -114.750528 & -114.749480 & -114.748886 & -114.749348 \\
\hline 2.0 & 1.8 & -114.750464 & -114.760951 & -114.759851 & -114.759232 & -114.759873 \\
\hline 2.0 & 2.0 & -114.773278 & -114.784813 & -114.783532 & -114.782837 & -114.784022 \\
\hline 2.0 & 2.25 & -114.795530 & -114.807951 & -114.806436 & -114.805681 & -114.807398 \\
\hline 2.0 & 2.5 & -114.814010 & -114.826644 & -114.824945 & -114.824193 & -114.826189 \\
\hline 2.0 & 2.75 & -114.828303 & -114.840755 & -114.838917 & -114.838201 & -114.840300 \\
\hline 2.0 & 3.0 & -114.838300 & -114.850469 & -114.848504 & -114.847826 & -114.849959 \\
\hline 2.0 & 3.5 & -114.847806 & -114.859637 & -114.857346 & -114.856715 & -114.858931 \\
\hline 2.0 & 4.0 & -114.848494 & -114.860501 & -114.857672 & -114.857044 & -114.859478 \\
\hline 2.0 & 5.0 & -114.841550 & -114.855385 & -114.850579 & -114.849844 & -114.853017 \\
\hline 2.0 & 6.0 & -114.835919 & -114.852798 & -114.845104 & -114.844161 & -114.848019 \\
\hline 2.0 & 8.0 & -114.832442 & -114.854060 & -114.841850 & -114.840639 & -114.845143 \\
\hline 2.2 & 1.2 & -114.593007 & -114.601086 & -114.600215 & -114.599780 & -114.599337 \\
\hline 2.2 & 1.4 & -114.748697 & -114.757464 & -114.756518 & -114.756028 & -114.755812 \\
\hline 2.2 & 1.6 & -114.817314 & -114.826950 & -114.825904 & -114.825348 & -114.825491 \\
\hline 2.2 & 1.7325 & -114.840232 & -114.850569 & -114.849432 & -114.848825 & -114.849296 \\
\hline 2.2 & 1.8 & -114.848287 & -114.859019 & -114.857825 & -114.857190 & -114.857860 \\
\hline 2.2 & 2.0 & -114.865144 & -114.877117 & -114.875713 & -114.874988 & -114.876307 \\
\hline 2.2 & 2.25 & -114.881158 & -114.894394 & -114.892679 & -114.891865 & -114.893846 \\
\hline 2.2 & 2.5 & -114.895538 & -114.909274 & -114.907293 & -114.906461 & -114.908844 \\
\hline 2.2 & 2.75 & -114.907472 & -114.921129 & -114.918948 & -114.918153 & -114.920699 \\
\hline 2.2 & 3.0 & -114.916234 & -114.929604 & -114.927253 & -114.926506 & -114.929103 \\
\hline 2.2 & 3.5 & -114.925154 & -114.938080 & -114.935341 & -114.934659 & -114.937330 \\
\hline 2.2 & 4.0 & -114.926439 & -114.939432 & -114.936107 & -114.935441 & -114.938314 \\
\hline 2.2 & 5.0 & -114.921317 & -114.935865 & -114.930571 & -114.929821 & -114.933393 \\
\hline 2.2 & 6.0 & -114.916803 & -114.933872 & -114.925982 & -114.925073 & -114.929295 \\
\hline 2.2 & 8.0 & -114.913920 & -114.934678 & -114.923073 & -114.921987 & -114.926851 \\
\hline
\end{tabular}


Table S4. (continued)

\begin{tabular}{|c|c|c|c|c|c|c|}
\hline$R_{\mathrm{Be}-\mathrm{F}}$ & $R_{\mathrm{H}-\mathrm{F}}$ & CCSD & $\operatorname{CCSD}(\mathrm{T})$ & R-CCSD $(\mathrm{T})$ & CR-CCSD(T) & $\operatorname{MRCI}(\mathrm{Q})$ \\
\hline 2.4 & 1.2 & -114.655705 & -114.663880 & -114.662946 & -114.662507 & -114.662008 \\
\hline 2.4 & 1.4 & -114.808625 & -114.817494 & -114.816481 & 15986 & 15763 \\
\hline 2.4 & 1.6 & -114.873339 & -114.883087 & -114.881968 & -114.881405 & -114.881532 \\
\hline 2.4 & 1.7325 & -114.893084 & -114.903551 & -114.902337 & -114.901722 & -114.902173 \\
\hline 2.4 & 1.8 & -114.899373 & -114.910253 & -114.908980 & -114.908336 & -114.908987 \\
\hline 2.4 & 2.0 & -114.910700 & -114.922961 & -114.921457 & -114.920716 & -114.922053 \\
\hline 2.4 & 2.25 & -114.920386 & -114.934299 & -114.932410 & -114.931548 & -114.933693 \\
\hline 2.4 & 2.5 & -114.930331 & -114.945132 & -114.942872 & -114.941959 & -114.944663 \\
\hline 2.4 & 2.75 & -114.939630 & -114.954536 & -114.951990 & -114.951107 & -114.954066 \\
\hline 2.4 & 3.0 & -114.946951 & -114.961599 & -114.958822 & -114.957998 & -114.961041 \\
\hline 2.4 & 3.5 & -114.954983 & -114.969085 & -114.965847 & -114.965112 & -114.968228 \\
\hline 2.4 & 4.0 & -114.956648 & -114.970687 & -114.966822 & -114.966119 & -114.969415 \\
\hline 2.4 & 5.0 & -114.953055 & -114.968343 & -114.962557 & -114.961800 & -114.965739 \\
\hline 2.4 & 6.0 & -114.949497 & -114.966838 & -114.958740 & -114.957871 & -114.962420 \\
\hline 2.4 & 8.0 & -114.9 & -114. & -114 . & -114 & -114 \\
\hline 2.5 & 1.2 & -114.675897 & -114.684106 & -114.683145 & -114.682706 & -114.682175 \\
\hline 2.5 & 1.4 & -114.827643 & -114.836547 & -114.835504 & -114.835008 & -114.834749 \\
\hline 2.5 & 1.6 & 0683 & -114. & $-114 . \varepsilon$ & -114 . & -114 \\
\hline 2.5 & 1.7325 & -114.909036 & -114 & -114 & -114 & -114 \\
\hline 2.5 & 1.8 & -114.914531 & -114.925449 & -114.924143 & -114.923497 & -114.924101 \\
\hline 2.5 & 2.0 & -114.923279 & -114.935621 & -114.934080 & -114.933334 & -114.934657 \\
\hline 2.5 & 2.25 & -114.9 & -114 & -114 & -114. & -114 \\
\hline 2.5 & 2.5 & -114.937388 & -114 & 50294 & -114 . & -114 \\
\hline 2.5 & 2.75 & -114.945261 & -114.960793 & -114.958064 & -114.957134 & -114.960297 \\
\hline 2.5 & 3.0 & -114.951790 & -114.967098 & -114.964101 & -114.963234 & -114.966507 \\
\hline 2.5 & 3.5 & -114.9 & -114 & -114 & -114. & -114 . \\
\hline 2.5 & 4.0 & -114.961061 & -114. & -114.971493 & -114.970773 & -114.974290 \\
\hline 2.5 & 5.0 & -114.958116 & -114.973782 & -114.967752 & -114.966995 & -114.971120 \\
\hline 2.5 & 6.0 & -114.954975 & -114.972484 & -114.964276 & -114.963429 & -114.968140 \\
\hline 2.5 & 8.0 & -114.9 & -114 & -114.961851 & -114.960934 & -114. \\
\hline 2.5719 & 1.2 & -114.687276 & -114.6 & -114.694526 & -114.694086 & -114.693533 \\
\hline 2.5719 & 1.4 & -114.838250 & -114.847172 & -114.846111 & -114.845614 & -114.845329 \\
\hline 2.5719 & 1.6 & -114.900188 & -114.909983 & -114.908813 & -114.908249 & -114.908307 \\
\hline 2.5719 & 1.7325 & -114.917613 & -114.928125 & -114.926858 & -114.926242 & -114.926609 \\
\hline 2.5719 & 1.8 & -114.922575 & -114.933505 & -114.932178 & -114.931533 & -114.932097 \\
\hline 2.5719 & 2.0 & -114.929551 & -114.941926 & -114.940362 & -114.939616 & -114.940914 \\
\hline 2.5719 & 2.25 & -114.933792 & -114.948118 & -114.946118 & -114.945231 & -114.947449 \\
\hline 2.5719 & 2.5 & -114.939636 & -114.955264 & -114.952788 & -114.951812 & -114.954745 \\
\hline 2.5719 & 2.75 & -114.946433 & -114.962413 & -114.959553 & -114.958590 & -114.961893 \\
\hline 2.5719 & 3.0 & -114.952363 & -114.968152 & -114.964995 & -114.964096 & -114.967534 \\
\hline 2.5719 & 3.5 & -114.959427 & -114.974592 & -114.970897 & -114.970114 & -114.973636 \\
\hline 2.5719 & 4.0 & -114.961261 & -114.976234 & -114.971883 & -114.971150 & -114.974830 \\
\hline 2.5719 & 5.0 & -114.958736 & -114.974676 & -114.968473 & -114.967718 & -114.971977 \\
\hline 2.5719 & 6.0 & -114.955870 & -114.973513 & -114.965224 & -114.964394 & -114.969219 \\
\hline 2.5719 & 8.0 & -114.953843 & -114.973872 & -114.962907 & -114.962026 & -114.967442 \\
\hline 2.6 & 1.2 & -114.691143 & -114.699376 & -114.698392 & -114.697952 & -114.697167 \\
\hline 2.6 & 1.4 & -114.841830 & -114.850757 & -114.849689 & -114.849192 & -114.848897 \\
\hline 2.6 & 1.6 & -114.903358 & -114.913157 & -114.911979 & -114.911415 & -114.911109 \\
\hline
\end{tabular}


Table S4. (continued)

\begin{tabular}{|c|c|c|c|c|c|c|}
\hline$R_{\mathrm{Be}-\mathrm{F}}$ & $R_{\mathrm{H}-\mathrm{F}}$ & CCSD & $\operatorname{CCSD}(\mathrm{T})$ & R-CCSD $(\mathrm{T})$ & CR-CCSD(T) & MRCI(Q) \\
\hline 2.6 & 1.7325 & -114.920437 & -114.930951 & -114.929677 & -114.929061 & -114.929137 \\
\hline 2.6 & 1.8 & -114.925199 & -114.936130 & -114.934796 & -114.934151 & -114.934699 \\
\hline 2.6 & 2.0 & -114.931502 & -114.943883 & -114.942312 & -114.941566 & -114.942852 \\
\hline 2.6 & 2.25 & -114.934858 & -114.949233 & -114.947219 & -114.946330 & -114.948553 \\
\hline 2.6 & 2.5 & -114.940004 & -114.955754 & -114.953246 & -114.952261 & -114.955226 \\
\hline 2.6 & 2.75 & -114.946367 & -114.962520 & -114.959610 & -114.958633 & -114.961990 \\
\hline 2.6 & 3.0 & -114.952055 & -114.968034 & -114.964814 & -114.963902 & -114.967405 \\
\hline 2.6 & 3.5 & -114.958944 & -114.974287 & -114.970516 & -114.969725 & -114.973315 \\
\hline 2.6 & 4.0 & -114.960793 & -114.975921 & -114.971490 & -114.970753 & -114.974496 \\
\hline 2.6 & 5.0 & -114.958422 & -114.974470 & -114.968201 & -114.967445 & -114.971757 \\
\hline 2.6 & 6.0 & -114.955657 & -114.973357 & -114.965035 & -114.964211 & -114.969081 \\
\hline 2.6 & 8.0 & -114.953683 & -114.973699 & -114.962759 & -114.961892 & -114.967347 \\
\hline 2.7 & 1.2 & -114.702748 & -114.710994 & -114.709991 & -114.709551 & -114.709487 \\
\hline 2.7 & 1.4 & -114.852476 & -114.861414 & -114.860324 & -114.859828 & -114.859499 \\
\hline 2.7 & 1.6 & -114.912635 & -114.922437 & -114.921236 & -114.920673 & -114.920677 \\
\hline 2.7 & 1.7325 & -114.928552 & -114.939061 & -114.937764 & -114.937149 & -114.937451 \\
\hline 2.7 & 1.8 & -114.932639 & -114.943561 & -114.942205 & -114.941561 & -114.942048 \\
\hline 2.7 & 2.0 & -114.936641 & -114.949020 & -114.947429 & -114.946685 & -114.947913 \\
\hline 2.7 & 2.25 & -114.936870 & -114.951377 & -114.949325 & -114.948431 & -114.950656 \\
\hline 2.7 & 2.5 & -114.939462 & -114.955613 & -114.953005 & -114.951991 & -114.955048 \\
\hline 2.7 & 2.75 & -114.944220 & -114.960978 & -114.957897 & -114.956872 & -114.960410 \\
\hline 2.7 & 3.0 & -114.949014 & -114.965670 & -114.962229 & -114.961268 & -114.964995 \\
\hline 2.7 & 3.5 & -114.9 & -114.971230 & -114.967186 & -114.966365 & -114.970196 \\
\hline 2.7 & 4.0 & -114.957118 & -114.972805 & -114.968087 & -114.967334 & -114.971304 \\
\hline 2.7 & 5.0 & -114.955249 & -114.971688 & -114.965180 & -114.964428 & -114.968927 \\
\hline 2.7 & 6.0 & -114.952822 & -114.970739 & -114.962296 & -114.961495 & -114.966523 \\
\hline 2.7 & 8.0 & -114. & -114.971036 & -114.960162 & -114.959339 & -114.964929 \\
\hline 2.9 & 1.2 & -114.718729 & -114.726969 & -114.725940 & -114.725502 & -114.724859 \\
\hline 2.9 & 1.4 & -114.866790 & -114.875712 & -114.874593 & -114.874099 & -114.873707 \\
\hline 2.9 & 1.6 & -114.924584 & -114.934349 & -114.933116 & -114.932557 & -114.932477 \\
\hline 2.9 & 1.7325 & -114.938482 & -114.948928 & -114.947601 & -114.946992 & -114.947183 \\
\hline 2.9 & 1.8 & -114.941385 & -114.952228 & -114.950843 & -114.950206 & -114.950566 \\
\hline 2.9 & 2.0 & -114.941243 & -114.953509 & -114.951900 & -114.951168 & -114.952219 \\
\hline 2.9 & 2.25 & -114.935420 & -114.949968 & -114.947895 & -114.947010 & -114.949167 \\
\hline 2.9 & 2.5 & -114.932594 & -114.949326 & -114.946587 & -114.945541 & -114.948678 \\
\hline 2.9 & 2.75 & -114.933786 & -114.951666 & -114.948284 & -114.947169 & -114.951003 \\
\hline 2.9 & 3.0 & -114.936593 & -114.954618 & -114.950746 & -114.949679 & -114.953825 \\
\hline 2.9 & 3.5 & -114.941333 & -114.958658 & -114.954063 & -114.953171 & -114.957482 \\
\hline 2.9 & 4.0 & -114.943140 & -114.959985 & -114.954690 & -114.953901 & -114.958329 \\
\hline 2.9 & 5.0 & -114.942080 & -114.959337 & -114.952357 & -114.951613 & -114.956483 \\
\hline 2.9 & 6.0 & -114.940222 & -114.958661 & -114.949951 & -114.949192 & -114.954527 \\
\hline 2.9 & 8.0 & -114.938723 & -114.958930 & -114.948079 & -114.947330 & -114.953179 \\
\hline 3.1 & 1.2 & -114.729047 & -114.737234 & -114.736197 & -114.735762 & -114.735441 \\
\hline 3.1 & 1.4 & -114.875724 & -114.884582 & -114.883452 & -114.882962 & -114.882677 \\
\hline 3.1 & 1.6 & -114.931578 & -114.941252 & -114.940006 & -114.939454 & -114.939297 \\
\hline 3.1 & 1.7325 & -114.943822 & -114.954144 & -114.952806 & -114.952205 & -114.952292 \\
\hline 3.1 & 1.8 & -114.945748 & -114.956445 & -114.955052 & -114.954425 & -114.954655 \\
\hline 3.1 & 2.0 & -114.942109 & -114.954146 & -114.952545 & -114.951830 & -114.952657 \\
\hline 3.1 & 2.25 & -114.930723 & -114.945033 & -114.943001 & -114.942144 & -114.944158 \\
\hline
\end{tabular}


Table S4. (continued)

\begin{tabular}{|c|c|c|c|c|c|c|}
\hline$R_{\mathrm{Be}-\mathrm{F}}$ & $R_{\mathrm{H}-\mathrm{F}}$ & CCSD & $\operatorname{CCSD}(\mathrm{T})$ & $\mathrm{R}-\mathrm{CCSD}(\mathrm{T})$ & CR-CCSD $(\mathrm{T})$ & $\operatorname{MRCI}(\mathrm{Q})$ \\
\hline 3.1 & 2.5 & -114.922171 & -114.939092 & -114.936345 & -114.935307 & -114.938352 \\
\hline 3.1 & 2.75 & -114.919203 & -114.937979 & -114.934394 & -114.933216 & -114.937212 \\
\hline 3.1 & 3.0 & -114.919662 & -114.939032 & -114.934767 & -114.933588 & -114.938086 \\
\hline 3.1 & 3.5 & -114.922653 & -114.941412 & -114.936255 & -114.935274 & -114.940048 \\
\hline 3.1 & 4.0 & -114.924261 & -114.942341 & -114.936460 & -114.935629 & -114.940509 \\
\hline 3.1 & 5.0 & -114.923792 & -114.941941 & -114.934480 & -114.933744 & -114.938979 \\
\hline 3.1 & 6.0 & -114.922380 & -114.941476 & -114.932450 & -114.931727 & -114.937361 \\
\hline 3.1 & 8.0 & -114.921122 & -114.941779 & -114.930811 & -114.930118 & -114.936211 \\
\hline 3.3 & 1.2 & -114.736447 & -114.744531 & -114.743501 & -114.743072 & -114.742622 \\
\hline 3.3 & 1.4 & -114.881990 & -114.890729 & -114.889605 & -114.889122 & -114.888623 \\
\hline 3.3 & 1.6 & -114.936269 & -114.945794 & -114.944555 & -114.944011 & -114.943781 \\
\hline 3.3 & 1.7325 & -114.947177 & -114.957316 & -114.955986 & -114.955398 & -114.955387 \\
\hline 3.3 & 1.8 & -114.948316 & -114.958805 & -114.957423 & -114.956810 & -114.956928 \\
\hline 3.3 & 2.0 & -114.941831 & -114.953556 & -114.951983 & -114.951289 & -114.951899 \\
\hline 3.3 & 2.25 & -114.925638 & -114.939494 & -114.937542 & -114.936722 & -114.938464 \\
\hline 3.3 & 2.5 & -114.911375 & -114.928024 & -114.925392 & -114.924403 & -114.927200 \\
\hline 3.3 & 2.75 & -114.903565 & -114.922820 & -114.919194 & -114.918009 & -114.921962 \\
\hline 3.3 & 3.0 & -114.901127 & -114.921709 & -114.917136 & -114. & 20603 \\
\hline 3.3 & 3.5 & -114.902017 & -114.922333 & 16598 & 5503 & 20709 \\
\hline 3.3 & 4.0 & -114.903317 & -114.922753 & -114.916261 & -114.915372 & -114.920689 \\
\hline 3.3 & 5.0 & -114.903277 & -114.922420 & -114.914451 & -114.913719 & -114.919311 \\
\hline 3.3 & 6.0 & -114.902213 & -114.922115 & -114.912712 & -114. & -114.917941 \\
\hline 3.3 & 8.0 & -114.901149 & -114.922490 & 11277 & -114.910624 & -114.916948 \\
\hline 3.5 & 1.2 & -114.742300 & -114.750229 & -114.749219 & -114.748797 & -114.748228 \\
\hline 3.5 & 1.4 & -114.886926 & -114.895493 & -114.894389 & -114.893915 & -114.893366 \\
\hline 3.5 & 1.6 & -114.939944 & -114.949266 & -114.948049 & -114.947517 & -114.947224 \\
\hline 3.5 & 1.7325 & -114.949794 & -114.959694 & -114.958389 & -114.957815 & -114.957724 \\
\hline 3.5 & 1.8 & -114.950312 & -114.960539 & -114.959184 & -114.958587 & -114.958610 \\
\hline 3.5 & 2.0 & -114.941589 & -114.952945 & -114.951413 & -114.950742 & -114.951176 \\
\hline 3.5 & 2.25 & -114.921509 & -114 & -114.932925 & -114.932144 & -114.933462 \\
\hline 3.5 & 2.5 & -114.902040 & -114.918010 & -114.915574 & -114.914651 & -114.917200 \\
\hline 3.5 & 2.75 & -114.888864 & -114.907975 & -114.904521 & -114.903398 & -114.907014 \\
\hline 3.5 & 3.0 & -114.882736 & -114.904168 & -114.899462 & -114.898150 & -114.902958 \\
\hline 3.5 & 3.5 & -114.880954 & -114.902972 & -114.896632 & -114. & -114.900988 \\
\hline 3.5 & 4.0 & -114.881854 & -114.902823 & -114.895665 & -114.894691 & -114.900423 \\
\hline 3.5 & 5.0 & -114.882131 & -114.902403 & -114.893876 & -114.893139 & -114.899077 \\
\hline 3.5 & 6.0 & -114.881342 & -114.902206 & -114.892354 & -114.891680 & -114.897883 \\
\hline 3.5 & 8.0 & -114.880436 & -114.902673 & -114.891097 & -114.890470 & -114.897013 \\
\hline 3.7 & 1.2 & -114.747246 & -114.754976 & -114.753996 & -114.753584 & -114.752908 \\
\hline 3.7 & 1.4 & -114.891142 & -114.899491 & -114.898417 & -114.897955 & -114.897199 \\
\hline 3.7 & 1.6 & -114.943167 & -114.952239 & -114.951055 & -114.950536 & -114.950189 \\
\hline 3.7 & 1.7325 & -114.952190 & -114.961809 & -114.960540 & -114.959982 & -114.959820 \\
\hline 3.7 & 1.8 & -114.952228 & -114.962151 & -114.960834 & -114.960255 & -114.960198 \\
\hline 3.7 & 2.0 & -114.941792 & -114.952745 & -114.951265 & -114.950618 & -114.950917 \\
\hline 3.7 & 2.25 & -114.918740 & -114.931390 & -114.929627 & -114.928883 & -114.929835 \\
\hline 3.7 & 2.5 & -114.895005 & -114.910071 & -114.907841 & -114.906981 & -114.909358 \\
\hline 3.7 & 2.75 & -114.876492 & -114.894796 & -114.891689 & -114.890671 & -114.893632 \\
\hline 3.7 & 3.0 & -114.865750 & -114.887334 & -114.882795 & -114.881545 & -114.886118 \\
\hline 3.7 & 3.5 & -114.860312 & -114.884139 & -114.877181 & -114.875797 & -114.881722 \\
\hline
\end{tabular}


Table S4. (continued)

\begin{tabular}{|c|c|c|c|c|c|c|}
\hline$R_{\mathrm{Be}-\mathrm{F}}$ & $R_{\mathrm{H}-\mathrm{F}}$ & CCSD & $\operatorname{CCSD}(\mathrm{T})$ & $\mathrm{R}-\mathrm{CCSD}(\mathrm{T})$ & CR-CCSD $(\mathrm{T})$ & $\operatorname{MRCI}(\mathrm{Q})$ \\
\hline 3.7 & 4.0 & -114.860702 & -114.883459 & -114.875534 & -114.874441 & -114.880560 \\
\hline 3.7 & 5.0 & -114.861237 & -114.882815 & -114.873649 & -114.872892 & -114.879163 \\
\hline 3.7 & 6.0 & -114.860674 & -114.882673 & -114.872282 & -114.871619 & -114.878088 \\
\hline 3.7 & 8.0 & -114.859897 & -114.883234 & -114.871180 & -114.870569 & -114.877313 \\
\hline 3.9 & 1.2 & -114.751550 & -114.759053 & -114.758105 & -114.757704 & -114.757455 \\
\hline 3.9 & 1.4 & -114.894874 & -114.902975 & -114.901937 & -114.901488 & -114.901424 \\
\hline 3.9 & 1.6 & -114.946127 & -114.954919 & -114.953774 & -114.953271 & -114.953456 \\
\hline 3.9 & 1.7325 & -114.954514 & -114.963823 & -114.962597 & -114.962056 & -114.961835 \\
\hline 3.9 & 1.8 & -114.954186 & -114.963779 & -114.962507 & -114.961947 & -114.961821 \\
\hline 3.9 & 2.0 & -114.942462 & -114.953001 & -114.951576 & -114.950953 & -114.951150 \\
\hline 3.9 & 2.25 & -114.917229 & -114.929270 & -114.927592 & -114.926884 & -114.927600 \\
\hline 3.9 & 2.5 & -114.890313 & -114.904441 & -114.902384 & -114.901577 & -114.903260 \\
\hline 3.9 & 2.75 & -114.867239 & -114.884322 & -114.881596 & -114.880672 & -114.883006 \\
\hline 3.9 & 3.0 & -114.851313 & -114.872114 & -114.868082 & -114.866978 & -114.870774 \\
\hline 3.9 & 3.5 & -114.840606 & -114.866154 & -114.858671 & -114.857178 & -114.863376 \\
\hline 3.9 & 4.0 & -114.840295 & -114.865177 & -114.856333 & -114.855083 & -114.861564 \\
\hline 3.9 & 5.0 & -114.841074 & -114.864201 & -114.854270 & -114.853473 & -114.860063 \\
\hline 3.9 & 6.0 & -114.840709 & -114.864050 & -114.853008 & 2343 & -114.859069 \\
\hline 3.9 & 8.0 & -114.840044 & -114.864697 & -114.852039 & 51434 & -114.858366 \\
\hline 4.1 & 1.2 & -114.755300 & -114.762565 & -114.761652 & -114.761263 & -114.760858 \\
\hline 4.1 & 1.4 & -114.898182 & -114.906024 & -114.905023 & -114.904587 & -114.904362 \\
\hline 4.1 & 1.6 & -114. & -114.957346 & -114.956241 & -114.955755 & -114.955759 \\
\hline 4.1 & 1.7325 & -114.956748 & -114.965739 & -114.964557 & -114.964035 & -114.964214 \\
\hline 4.1 & 1.8 & -114.956143 & -114.965401 & -114.964175 & -114.963634 & -114.963919 \\
\hline 4.1 & 2.0 & -114.943462 & -114.953596 & -114.952225 & -114.951628 & -114.951738 \\
\hline 4.1 & 2.25 & -114.916663 & -114.9 & -114. & -114.925864 & -114.926427 \\
\hline 4.1 & 2.5 & -114.887526 & -114.900800 & -114.898877 & -114.898115 & -114.899312 \\
\hline 4.1 & 2.75 & -114.861109 & -114.876916 & -114.874484 & -114.873624 & -114.876481 \\
\hline 4.1 & 3.0 & -114.840394 & -114.859705 & -114.856309 & -114.855341 & -114.857633 \\
\hline 4.1 & 3.5 & -114.822275 & -114 & -114 & -114. & -114.846211 \\
\hline 4.1 & 4.0 & -114.820834 & -114.848237 & -114.838287 & -114.836853 & -114.843680 \\
\hline 4.1 & 5.0 & -114.821889 & -114.846897 & -114.836016 & -114.835152 & -114.842050 \\
\hline 4.1 & 6.0 & -114.821717 & -114.846658 & -114.834816 & -114.834136 & -114.841108 \\
\hline 4.1 & 8.0 & -114.821152 & -114.847367 & -114.833960 & -114.833354 & -114.840463 \\
\hline 4.5 & 1.2 & -114.761249 & -114.768061 & -114.767210 & -114.766844 & -114.765060 \\
\hline 4.5 & 1.4 & -114.903539 & -114.910887 & -114.909954 & -114.909545 & -114.907577 \\
\hline 4.5 & 1.6 & -114.953411 & -114.961366 & -114.960337 & -114.959881 & -114.959601 \\
\hline 4.5 & 1.7325 & -114.960678 & -114.969076 & -114.967975 & -114.967487 & -114.967363 \\
\hline 4.5 & 1.8 & -114.959712 & -114.968348 & -114.967208 & -114.966704 & -114.966663 \\
\hline 4.5 & 2.0 & -114.945806 & -114.955212 & -114.953940 & -114.953386 & -114.953608 \\
\hline 4.5 & 2.25 & -114.917099 & -114.927630 & -114.926157 & -114.925539 & -114.926114 \\
\hline 4.5 & 2.5 & -114.885459 & -114.897397 & -114.895658 & -114.894968 & -114.895820 \\
\hline 4.5 & 2.75 & -114.855484 & -114.869292 & -114.867177 & -114.866401 & -114.867805 \\
\hline 4.5 & 3.0 & -114.829148 & -114.845534 & -114.842863 & -114.841990 & -114.844060 \\
\hline 4.5 & 3.5 & -114.793076 & -114.817687 & -114.812134 & -114.811117 & -114.816019 \\
\hline 4.5 & 4.0 & -114.785050 & -114.817915 & -114.805771 & -114.804120 & -114.811660 \\
\hline 4.5 & 5.0 & -114.786755 & -114.817148 & -114.803441 & -114.802327 & -114.809769 \\
\hline 4.5 & 6.0 & -114.787068 & -114.816348 & -114.802252 & -114.801486 & -114.808918 \\
\hline 4.5 & 8.0 & -114.786708 & -114.817048 & -114.801572 & -114.800936 & -114.808354 \\
\hline
\end{tabular}


Table S4. (continued)

\begin{tabular}{|c|c|c|c|c|c|c|}
\hline$R_{\mathrm{Be}-\mathrm{F}}$ & $R_{\mathrm{H}-\mathrm{F}}$ & CCSD & $\operatorname{CCSD}(\mathrm{T})$ & R-CCSD $(\mathrm{T})$ & CR-CCSD(T) & MRCI(Q) \\
\hline 4.7 & 1.2 & -114.763501 & -114.770117 & -114.769292 & -114.768936 & -114.767150 \\
\hline 4.7 & 1.4 & -114.905599 & -114.912733 & -114.911828 & -114.911431 & -114.909462 \\
\hline 4.7 & 1.6 & -114.955217 & -114.962934 & -114.961937 & -114.961495 & -114.961104 \\
\hline 4.7 & 1.7325 & -114.962280 & -114.970422 & -114.969355 & -114.968883 & -114.968643 \\
\hline 4.7 & 1.8 & -114.961199 & -114.969569 & -114.968464 & -114.967976 & -114.967818 \\
\hline 4.7 & 2.0 & -114.946911 & -114.956011 & -114.954780 & -114.954246 & -114.954341 \\
\hline 4.7 & 2.25 & -114.917638 & -114.927791 & -114.926368 & -114.925773 & -114.926199 \\
\hline 4.7 & 2.5 & -114.885321 & -114.896764 & -114.895089 & -114.894427 & -114.895165 \\
\hline 4.7 & 2.75 & -114.854511 & -114.867624 & -114.865597 & -114.864854 & -114.865803 \\
\hline 4.7 & 3.0 & -114.827019 & -114.842401 & -114.839861 & -114.839012 & -114.839868 \\
\hline 4.7 & 3.5 & -114.785007 & -114.807512 & -114.803032 & -114.802030 & -114.799505 \\
\hline 4.7 & 4.0 & -114.769059 & -114.803031 & -114.791161 & -114.789712 & -114.797583 \\
\hline 4.7 & 5.0 & -114.770744 & -114.805166 & -114.789283 & -114.787967 & -114.795600 \\
\hline 4.7 & 6.0 & -114.771425 & -114.803784 & -114.788057 & -114.787199 & -114.794811 \\
\hline 4.7 & 8.0 & -114.771197 & -114.804362 & -114.787437 & -114.786760 & -114.794287 \\
\hline 5.0 & 1.2 & -114.766101 & -114.772470 & -114.771677 & -114.771333 & -114.769549 \\
\hline 5.0 & 1.4 & -114.907997 & -114.914860 & -114.913991 & -114.913608 & -114.911643 \\
\hline 5.0 & 1.6 & -114.957347 & -114.964767 & -114.963809 & -114.963384 & -114.961256 \\
\hline 5.0 & .7325 & -114.964198 & -114.972019 & -114.970994 & -114.970541 & -114.968330 \\
\hline 5.0 & 1.8 & -114.962997 & -114.971034 & -114.969973 & -114.969505 & -114.969204 \\
\hline 5.0 & 2.0 & -114.948316 & -114.957037 & -114.955856 & -114.955345 & -114.955292 \\
\hline 5.0 & 2.25 & -114.918476 & -114.928172 & -114.926809 & -114.926243 & -114.926518 \\
\hline 5.0 & 2.5 & -114.885525 & -114.896391 & -114.894790 & -114.8 & -114.894764 \\
\hline 5.0 & 2.75 & -114.854015 & -114.866362 & -114.864430 & -114.863726 & -114.864606 \\
\hline 5.0 & 3.0 & -114.825724 & -114.840052 & -114.837628 & -114.836816 & -114.837840 \\
\hline 5.0 & 3.5 & -114.780528 & -114.801375 & -114.797038 & -114.795857 & -114.796799 \\
\hline 5.0 & 4.0 & -114.751305 & -114.782213 & -114.774060 & -114.772821 & -114.771813 \\
\hline 5.0 & 5.0 & -114.748388 & -114.791733 & -114.770985 & -114.769259 & -114.776881 \\
\hline 5.0 & 6.0 & -114.749930 & -114.789091 & -114.769760 & -114.768616 & -114.776264 \\
\hline 5.0 & 8.0 & -114.750008 & -114.789186 & -114.769184 & -114.768343 & -114.775832 \\
\hline 5.2 & 1.2 & -114.767397 & -114.773631 & -114.772855 & -114.772518 & -114.770736 \\
\hline 5.2 & 1.4 & -114.909198 & -114.915914 & -114.915064 & -114.914689 & -114.912728 \\
\hline 5.2 & 1.6 & -114.958425 & -114.965682 & -114.964745 & -114.964329 & -114.962209 \\
\hline 5.2 & 1.7325 & -114.965177 & -114.972824 & -114.971822 & -114.971379 & -114.969176 \\
\hline 5.2 & 1.8 & -114.963921 & -114.971777 & -114.970740 & -114.970282 & -114.969907 \\
\hline 5.2 & 2.0 & -114.949060 & -114.957577 & -114.956423 & -114.955924 & -114.955793 \\
\hline 5.2 & 2.25 & -114.918969 & -114.928423 & -114.927092 & -114.926540 & -114.926737 \\
\hline 5.2 & 2.5 & -114.885750 & -114.896321 & -114.894758 & -114.894149 & -114.894682 \\
\hline 5.2 & 2.75 & -114.853978 & -114.865949 & -114.864064 & -114.863381 & -114.864228 \\
\hline 5.2 & 3.0 & -114.825450 & -114.839283 & -114.836914 & -114.836124 & -114.837215 \\
\hline 5.2 & 3.5 & -114.779859 & -114.800011 & -114.795605 & -114.794383 & -114.795796 \\
\hline 5.2 & 4.0 & -114.748163 & -114.779775 & -114.770950 & -114.768919 & -114.771200 \\
\hline 5.2 & 5.0 & -114.734482 & -114.786418 & -114.761152 & -114.759082 & -114.766191 \\
\hline 5.2 & 6.0 & -114.736882 & -114.783318 & -114.760141 & -114.758570 & -114.765803 \\
\hline 5.2 & 8.0 & -114.737304 & -114.782721 & -114.759548 & -114.758399 & -114.765494 \\
\hline 5.5 & 1.2 & -114.768822 & -114.774894 & -114.774138 & -114.773809 & -114.772032 \\
\hline 5.5 & 1.4 & -114.910523 & -114.917062 & -114.916233 & -114.915868 & -114.913914 \\
\hline 5.5 & 1.6 & -114.959618 & -114.966680 & -114.965767 & -114.965363 & -114.963252 \\
\hline 5.5 & 1.7325 & -114.966266 & -114.973704 & -114.972728 & -114.972297 & -114.971571 \\
\hline
\end{tabular}


Table S4. (continued)

\begin{tabular}{|c|c|c|c|c|c|c|}
\hline$R_{\mathrm{Be}-\mathrm{F}}$ & $R_{\mathrm{H}-\mathrm{F}}$ & CCSD & $\operatorname{CCSD}(\mathrm{T})$ & R-CCSD $(\mathrm{T})$ & CR-CCSD $(\mathrm{T})$ & $\mathrm{MRCI}(\mathrm{Q})$ \\
\hline 5.5 & 1.8 & -114.964952 & -114.972591 & -114.971581 & -114.971137 & -114.970477 \\
\hline 5.5 & 2.0 & -114.949902 & -114.958176 & -114.957053 & -114.956569 & -114.956345 \\
\hline 5.5 & 2.25 & -114.919552 & -114.928720 & -114.927426 & -114.926892 & -114.926995 \\
\hline 5.5 & 2.5 & -114.886072 & -114.896299 & -114.894781 & -114.894193 & -114.894641 \\
\hline 5.5 & 2.75 & -114.854065 & -114.865614 & -114.863781 & -114.863122 & -114.863642 \\
\hline 5.5 & 3.0 & -114.825371 & -114.838662 & -114.836359 & -114.835594 & -114.836396 \\
\hline 5.5 & 3.5 & -114.779844 & -114.799102 & -114.794748 & -114.793528 & -114.795302 \\
\hline 5.5 & 4.0 & -114.748848 & -114.781482 & -114.770941 & -114.768366 & -114.771381 \\
\hline 5.5 & 5.0 & -114.717048 & -114.786767 & -114.754648 & -114.750403 & -114.754744 \\
\hline 5.5 & 6.0 & -114.719822 & -114.787513 & -114.752691 & -114.749276 & -114.757716 \\
\hline 5.5 & 8.0 & -114.721152 & -114.784196 & -114.751716 & -114.749063 & -114.757836 \\
\hline 6.0 & 1.2 & -114.770207 & -114.776089 & -114.775355 & -114.775037 & -114.773267 \\
\hline 6.0 & 1.4 & -114.911805 & -114.918135 & -114.917332 & -114.916978 & -114.915036 \\
\hline 6.0 & 1.6 & -114.960767 & -114.967600 & -114.966716 & -114.966324 & -114.964229 \\
\hline 6.0 & 1.7325 & -114.967311 & -114.974503 & -114.973559 & -114.973142 & -114.972329 \\
\hline 6.0 & 1.8 & -114.965940 & -114.973324 & -114.972347 & -114.971917 & -114.971159 \\
\hline 6.0 & 2.0 & -114.950708 & -114.958696 & -114.957610 & -114.957143 & -114.956574 \\
\hline 6.0 & 2.25 & -114.920114 & -114.928951 & -114.927699 & -114.927186 & -114.926893 \\
\hline 6.0 & 2.5 & -114.886403 & -114.896242 & -114.894773 & -114.894208 & -114.894214 \\
\hline 6.0 & 2.75 & -114.854216 & -114.865301 & -114.863529 & -114.862895 & -114.863225 \\
\hline 6.0 & 3.0 & -114.825441 & -114.838162 & -114.835936 & -114.835198 & -114.835924 \\
\hline 6.0 & 3.5 & -114.780292 & -114.798474 & -114.794333 & -114.793146 & -114.795161 \\
\hline 6.0 & 4.0 & -114.750682 & -114.780856 & -114.770990 & -114.768517 & -114.771944 \\
\hline 6.0 & 5.0 & -114.722852 & -114.832671 & -114.769729 & -114.756259 & -114.756115 \\
\hline 6.0 & 6.0 & -114.711222 & -114.886246 & -114.776296 & -114.756605 & -114.755168 \\
\hline 6.0 & 8.0 & -114.710479 & -114.857400 & -114.767577 & -114.754151 & -114.755253 \\
\hline 8.0 & 1.2 & -114.770933 & -114.776534 & -114.775834 & -114.775530 & -114.773790 \\
\hline 8.0 & 1.4 & -114.912368 & -114.918388 & -114.917623 & -114.917286 & -114.915380 \\
\hline 8.0 & 1.6 & -114.961144 & -114.967632 & -114.966791 & -114.966419 & -114.965513 \\
\hline 8.0 & 1.7325 & -114.967554 & -114.974377 & -114.973479 & -114.973083 & -114.972245 \\
\hline 8.0 & 1.8 & -114.966112 & -114.973113 & -114.972184 & -114.971777 & -114.970980 \\
\hline 8.0 & 2.0 & -114.950668 & -114.958228 & -114.957197 & -114.956757 & -114.956110 \\
\hline 8.0 & 2.25 & -114.919826 & -114.928171 & -114.926984 & -114.926501 & -114.926089 \\
\hline 8.0 & 2.5 & -114.885922 & -114.895196 & -114.893803 & -114.893272 & -114.893138 \\
\hline 8.0 & 2.75 & -114.853642 & -114.864079 & -114.862396 & -114.861798 & -114.861999 \\
\hline 8.0 & 3.0 & -114.824922 & -114.836891 & -114.834774 & -114.834072 & -114.834725 \\
\hline 8.0 & 3.5 & -114.780488 & -114.797367 & -114.793544 & -114.792409 & -114.794548 \\
\hline 8.0 & 4.0 & -114.752443 & -114.777979 & -114.770292 & -114.768179 & -114.772153 \\
\hline 8.0 & 5.0 & -114.728261 & -114.784981 & -114.758505 & -114.752133 & -114.757104 \\
\hline 8.0 & 6.0 & -114.721645 & -114.831395 & -114.766333 & -114.752480 & -114.754568 \\
\hline 8.0 & 8.0 & -114.718528 & -114.920089 & -114.782603 & -114.757570 & -114.754035 \\
\hline
\end{tabular}


Table S5. The CCSD, CCSD(T), R-CCSD(T), CR-CCSD(T), and MRCI(Q) energies, in hartree, of the BeFH system, as described by the cc-pVTZ basis set, for the Be-F-H angle $\theta=70^{\circ}$. The Be $-\mathrm{F}$ and $\mathrm{H}-\mathrm{F}$ bond distances, $R_{\mathrm{Be}-\mathrm{F}}$ and $R_{\mathrm{H}-\mathrm{F}}$, respectively, are in bohr.

\begin{tabular}{|c|c|c|c|c|c|c|}
\hline$R_{\mathrm{Be}-\mathrm{F}}$ & $R_{\mathrm{H}-\mathrm{F}}$ & CCSD & $\operatorname{CCSD}(\mathrm{T})$ & R-CCSD $(\mathrm{T})$ & CR-CCSD(T) & MRCI(Q) \\
\hline 1.8 & 1.2 & -114.247332 & -114.255231 & -114.254503 & -114.254074 & -114.253784 \\
\hline 1.8 & 1.4 & -114.413782 & -114.422371 & -114.421579 & -114.421097 & -114.421068 \\
\hline 1.8 & 1.6 & -114.500253 & -114.509646 & -114.508774 & -114.508229 & -114.508560 \\
\hline 1.8 & 1.7325 & -114.537688 & -114.547624 & -114.546691 & -114.546105 & -114.546705 \\
\hline 1.8 & 1.8 & -114.553511 & -114.563701 & -114.562737 & -114.562130 & -114.562867 \\
\hline 1.8 & 2.0 & -114.592731 & -114.603477 & -114.602433 & -114.601783 & -114.602863 \\
\hline 1.8 & 2.25 & -114.631063 & -114.641984 & -114.640881 & -114.640218 & -114.641518 \\
\hline 1.8 & 2.5 & -114.659795 & -114.670477 & -114.669353 & -114.668712 & -114.670060 \\
\hline 1.8 & 2.75 & -114.679920 & -114.690225 & -114.689094 & -114.688486 & -114.689819 \\
\hline 1.8 & 3.0 & -114.692826 & -114.702783 & -114.701638 & -114.701060 & -114.702375 \\
\hline 1.8 & 3.5 & -114.703026 & -114.712589 & -114.711343 & -114.710806 & -114.712159 \\
\hline 1.8 & 4.0 & -114.700721 & -114.710315 & -114.708822 & -114.708303 & -114.709823 \\
\hline 1.8 & 5.0 & -114.684608 & -114.695491 & -114.692791 & -114.692238 & -114.694482 \\
\hline 1.8 & 6.0 & -114.672076 & -114.685983 & -114.680730 & -114.680011 & -114.683158 \\
\hline 1.8 & 8.0 & -114.664252 & -114.685353 & -114.673794 & -114.672623 & -114.676683 \\
\hline 1.9 & 1.2 & -114.365042 & -114.373038 & -114.372271 & -114.371838 & -114.371531 \\
\hline 1.9 & 1.4 & -114.528374 & -114.537079 & -114.536244 & -114.535755 & -114.535717 \\
\hline 1.9 & 1.6 & -114.610638 & -114.620185 & -114.619264 & -114.618710 & -114.619049 \\
\hline 1.9 & .7325 & -114.645079 & -114.655216 & -114.654227 & -114.653628 & -114.654261 \\
\hline 1.9 & 1.8 & -114.659415 & -114.669837 & -114.668814 & -114.668192 & -114.668977 \\
\hline 1.9 & 2.0 & -114.694724 & -114.705810 & -114.704691 & -114.704018 & -114.705194 \\
\hline 1.9 & 2.25 & -114.729619 & -114.740988 & -114.739792 & -114.739099 & -114.740543 \\
\hline 1.9 & 2.5 & -114.756427 & -114.767595 & -114.766368 & -114.765695 & -114.767201 \\
\hline 1.9 & 2.75 & -114.775673 & -114.786452 & -114.785215 & -114.784577 & -114.786063 \\
\hline 1.9 & 3.0 & -114.788309 & -114.798709 & -114.797458 & -114.796853 & -114.798312 \\
\hline 1.9 & 3.5 & -114.798763 & -114.808707 & -114.807354 & -114.806796 & -114.808278 \\
\hline 1.9 & 4.0 & -114.797058 & -114.806992 & -114.805382 & -114.804847 & -114.806492 \\
\hline 1.9 & 5.0 & -114.782124 & -114.793288 & -114.790439 & -114.789876 & -114.792255 \\
\hline 1.9 & 6.0 & -114.770314 & -114.784342 & -114.778982 & -114.778265 & -114.781554 \\
\hline 1.9 & 8.0 & -114.762914 & -114.783496 & -114.772264 & -114.771144 & -114.775375 \\
\hline 2.0 & 1.2 & -114.453487 & -114.461572 & -114.460765 & -114.460327 & -114.460002 \\
\hline 2.0 & 1.4 & -114.614178 & -114.622987 & -114.622109 & -114.621614 & -114.621563 \\
\hline 2.0 & 1.6 & -114.692711 & -114.702396 & -114.701426 & -114.700863 & -114.701209 \\
\hline 2.0 & 1.7325 & -114.724393 & -114.734710 & -114.733667 & -114.733056 & -114.733720 \\
\hline 2.0 & 1.8 & -114.737326 & -114.747960 & -114.746877 & -114.746242 & -114.747073 \\
\hline 2.0 & 2.0 & -114.768836 & -114.780247 & -114.779053 & -114.778357 & -114.779630 \\
\hline 2.0 & 2.25 & -114.800243 & -114.812061 & -114.810769 & -114.810044 & -114.811640 \\
\hline 2.0 & 2.5 & -114.824986 & -114.836655 & -114.835318 & -114.834611 & -114.836291 \\
\hline 2.0 & 2.75 & -114.843191 & -114.854467 & -114.853115 & -114.852445 & -114.854103 \\
\hline 2.0 & 3.0 & -114.855417 & -114.866284 & -114.864917 & -114.864283 & -114.865905 \\
\hline 2.0 & 3.5 & -114.865955 & -114.876302 & -114.874830 & -114.874250 & -114.875881 \\
\hline 2.0 & 4.0 & -114.864780 & -114.875074 & -114.873336 & -114.872783 & -114.874574 \\
\hline 2.0 & 5.0 & -114.851000 & -114.862464 & -114.859455 & -114.858881 & -114.861415 \\
\hline 2.0 & 6.0 & -114.839912 & -114.854087 & -114.848608 & -114.847894 & -114.851342 \\
\hline 2.0 & 8.0 & -114.832926 & -114.853076 & -114.842124 & -114.841053 & -114.845464 \\
\hline 2.2 & 1.2 & -114.570233 & -114.578466 & -114.577584 & -114.577139 & -114.576771 \\
\hline 2.2 & 1.4 & -114.726708 & -114.735691 & -114.734730 & -114.734225 & -114.734196 \\
\hline
\end{tabular}


Table S5. (continued)

\begin{tabular}{|c|c|c|c|c|c|c|}
\hline$R_{\mathrm{Be}-\mathrm{F}}$ & $R_{\mathrm{H}-\mathrm{F}}$ & CCSD & $\operatorname{CCSD}(\mathrm{T})$ & $\mathrm{R}-\mathrm{CCSD}(\mathrm{T})$ & CR-CCSD $(\mathrm{T})$ & MRCI(Q) \\
\hline 2.2 & 1.6 & -114.798981 & -114.808893 & -114.807829 & -114.807253 & -114.807656 \\
\hline 2.2 & 1.7325 & -114.825807 & -114.836424 & -114.835275 & -114.834645 & -114.835402 \\
\hline 2.2 & 1.8 & -114.836194 & -114.847181 & -114.845984 & -114.845326 & -114.846272 \\
\hline 2.2 & 2.0 & -114.860513 & -114.872505 & -114.871161 & -114.870426 & -114.871909 \\
\hline 2.2 & 2.25 & -114.884891 & -114.897591 & -114.896097 & -114.895309 & -114.897220 \\
\hline 2.2 & 2.5 & -114.905175 & -114.917883 & -114.916306 & -114.915528 & -114.917580 \\
\hline 2.2 & 2.75 & -114.920898 & -114.933232 & -114.931619 & -114.930880 & -114.932919 \\
\hline 2.2 & 3.0 & -114.931933 & -114.943809 & -114.942172 & -114.941478 & -114.943466 \\
\hline 2.2 & 3.5 & -114.942159 & -114.953378 & -114.951629 & -114.951004 & -114.952967 \\
\hline 2.2 & 4.0 & -114.941830 & -114.952903 & -114.950868 & -114.950278 & -114.952391 \\
\hline 2.2 & 5.0 & -114.930246 & -114.942365 & -114.939001 & -114.938404 & -114.941274 \\
\hline 2.2 & 6.0 & -114.920575 & -114.935118 & -114.929373 & -114.928663 & -114.932442 \\
\hline 2.2 & 8.0 & -114.914385 & -114.933907 & -114.923383 & -114.922407 & -114.927163 \\
\hline 2.4 & 1.2 & -114.636979 & -114.645320 & -114.644371 & -114.643922 & -114.643498 \\
\hline 2.4 & 1.4 & -114.790258 & -114.799365 & -114. & 97819 & 97720 \\
\hline 2.4 & 1.6 & -114.857576 & -114.867643 & -114.866495 & -114.865911 & -114.866251 \\
\hline 2.4 & 1.7325 & -114.880345 & -114.891166 & -114.889923 & -114.889282 & -114.890002 \\
\hline 2.4 & 1.8 & -114 & -114.899752 & -114 & 7782 & -114 \\
\hline 2.4 & 2.0 & 06242 & -114.918687 & 7209 & 6445 & 18019 \\
\hline 2.4 & 2.25 & -114.923621 & -114.937128 & -114.935436 & -114.934592 & -114.936745 \\
\hline 2.4 & 2.5 & -114.939114 & -114.952875 & -114.951040 & -114.950189 & -114.952580 \\
\hline 2.4 & 2.75 & -114 & -114.965395 & -114 & -114 & 65081 \\
\hline 2.4 & 3.0 & 61376 & -114.974352 & 72400 & 71642 & 973988 \\
\hline 2.4 & 3.5 & -114.970714 & -114.982893 & -114.980815 & -114.980142 & -114.982429 \\
\hline 2.4 & 4.0 & -114.970942 & -114.982864 & -114.980480 & -114.979854 & -114.982281 \\
\hline 2.4 & 5.0 & $\$ 1349$ & -114.974180 & -114 & -114 & -114 \\
\hline 2.4 & 6.0 & -114.953019 & -114.968012 & -114.961972 & 961269 & -114.965349 \\
\hline 2.4 & 8.0 & -114.947561 & -114.966743 & -114.956495 & -114.955606 & -114.960659 \\
\hline 2.5 & 1.2 & -114.658932 & -114.667311 & -114.666333 & -114.665883 & -114.665427 \\
\hline 2.5 & 1.4 & -114 & 20041 & -114 & -114 & -114 \\
\hline 2.5 & 1.6 & -114.876130 & -114.886247 & -114.885062 & -114.884475 & -114.884777 \\
\hline 2.5 & 1.7325 & -114.897138 & -114.908024 & -114.906741 & -114.906097 & -114.906782 \\
\hline 2.5 & 1.8 & -114.904326 & -114.915638 & -114.914298 & -114.913622 & -114.914530 \\
\hline 2.5 & 2.0 & -114.918985 & -114.931597 & -114.930061 & -114 & -114.930882 \\
\hline 2.5 & 2.25 & -114.932896 & -114.946759 & -114.944975 & -114.944107 & -114.946366 \\
\hline 2.5 & 2.5 & -114.945890 & -114.960165 & -114.958202 & -114.957315 & -114.959873 \\
\hline 2.5 & 2.75 & -114.957129 & -114.971172 & -114.969108 & -114.968259 & -114.970858 \\
\hline 2.5 & 3.0 & -114.965655 & -114.979210 & -114.977087 & -114.976295 & -114.978836 \\
\hline 2.5 & 3.5 & -114.974360 & -114.987051 & -114.984788 & -114.984091 & -114.986560 \\
\hline 2.5 & 4.0 & -114.974759 & -114.987131 & -114.984554 & -114.983910 & -114.986510 \\
\hline 2.5 & 5.0 & -114.966066 & -114.979272 & -114.975304 & -114.974675 & -114.978030 \\
\hline 2.5 & 6.0 & -114.958367 & -114.973611 & -114.967416 & -114.966717 & -114.970951 \\
\hline 2.5 & 8.0 & -114.953248 & -114.972357 & -114.962196 & -114.961345 & -114.966543 \\
\hline 2.5719 & 1.2 & -114.671479 & -114.679879 & -114.678883 & -114.678432 & -114.677953 \\
\hline 2.5719 & 1.4 & -114.822585 & -114.831754 & -114.830666 & -114.830154 & -114.829989 \\
\hline 2.5719 & 1.6 & -114.886465 & -114.896606 & -114.895398 & -114.894811 & -114.895081 \\
\hline 2.5719 & 1.7325 & -114.906309 & -114.917225 & -114.915916 & -114.915271 & -114.915927 \\
\hline 2.5719 & 1.8 & -114.912835 & -114.924185 & -114.922818 & -114.922141 & -114.923021 \\
\hline 2.5719 & 2.0 & -114.925390 & -114.938094 & -114.936522 & -114.935744 & -114.937340 \\
\hline
\end{tabular}


Table S5. (continued)

\begin{tabular}{|c|c|c|c|c|c|c|}
\hline$R_{\mathrm{Be}-\mathrm{F}}$ & $R_{\mathrm{H}-\mathrm{F}}$ & CCSD & $\operatorname{CCSD}(\mathrm{T})$ & $\mathrm{R}-\mathrm{CCSD}(\mathrm{T})$ & CR-CCSD $(\mathrm{T})$ & MRCI(Q) \\
\hline 2.5719 & 2.25 & -114.936827 & -114.950918 & -114.949074 & -114.948191 & -114.950513 \\
\hline 2.5719 & 2.5 & -114.947983 & -114.962617 & -114.960564 & -114.959652 & -114.962325 \\
\hline 2.5719 & 2.75 & -114.958039 & -114.972502 & -114.970325 & -114.969448 & -114.972189 \\
\hline 2.5719 & 3.0 & -114.965861 & -114.979844 & -114.977593 & -114.976776 & -114.979463 \\
\hline 2.5719 & 3.5 & -114.974043 & -114.987114 & -114.984712 & -114.983998 & -114.986604 \\
\hline 2.5719 & 4.0 & -114.974522 & -114.987227 & -114.984504 & -114.983847 & -114.986578 \\
\hline 2.5719 & 5.0 & -114.966434 & -114.979914 & -114.975793 & -114.975157 & -114.978638 \\
\hline 2.5719 & 6.0 & -114.959167 & -114.974603 & -114.968294 & -114.967597 & -114.971943 \\
\hline 2.5719 & 8.0 & -114.954280 & -114.973374 & -114.963256 & -114.962432 & -114.967731 \\
\hline 2.6 & 1.2 & -114.675781 & -114.684187 & -114.683185 & -114.682734 & -114.682245 \\
\hline 2.6 & 1.4 & -114.826571 & -114.835747 & -114.834651 & -114.834139 & -114.833972 \\
\hline 2.6 & 1.6 & -114.889950 & -114.900098 & -114.898881 & -114.898294 & -114.898556 \\
\hline 2.6 & 1.7325 & -114.909360 & -114.920285 & -114.918967 & -114.918322 & -114.918965 \\
\hline 2.6 & 1.8 & -114.915639 & -114.927000 & -114.925623 & -114.924945 & -114.925813 \\
\hline 2.6 & 2.0 & -114.927394 & -114.940128 & 38544 & 37764 & 39361 \\
\hline 2.6 & 2.25 & -114.937869 & -114.952043 & -114.950177 & -114.949289 & -114.951633 \\
\hline 2.6 & 2.5 & -114.948299 & -114.963069 & -114.960981 & -114.960060 & -114.962777 \\
\hline 2.6 & 2.75 & 57881 & -114.972508 & 70287 & 69400 & -114 \\
\hline 2.6 & 3.0 & 65419 & -114.979570 & 77270 & 76442 & 79188 \\
\hline 2.6 & 3.5 & -114.973382 & -114.986604 & -114.984147 & -114.983425 & -114.986086 \\
\hline 2.6 & 4.0 & -114.973882 & -114.986720 & -114.983939 & -114.983277 & -114.986060 \\
\hline 2.6 & 5.0 & 66021 & -114.979610 & 5428 & -114. & -114 \\
\hline 2.6 & 6.0 & -114.958919 & -114.974431 & -114.968077 & 967381 & 971771 \\
\hline 2.6 & 8.0 & -114.954119 & -114.973215 & -114.963110 & -114.962296 & -114.967634 \\
\hline 2.7 & 1.2 & -114.688845 & -114.697265 & -114.696242 & -114.695792 & -114.695821 \\
\hline 2.7 & 1.4 & -114 & -114.847775 & 46655 & 6143 & -114.845931 \\
\hline 2.7 & 1.6 & -114.900305 & -114.910464 & -114.909220 & -114.908634 & -114.908850 \\
\hline 2.7 & 1.7325 & -114.918267 & -114.929205 & -114.927859 & -114.927214 & -114.927805 \\
\hline 2.7 & 1.8 & -114.923710 & -114.935090 & -114.933682 & -114.933005 & -114.933822 \\
\hline 2.7 & 2.0 & -114 & -114.945536 & -114.943911 & 3129 & 44705 \\
\hline 2.7 & 2.25 & -114.939807 & -114.954239 & -114.952300 & -114.951397 & -114.953804 \\
\hline 2.7 & 2.5 & -114.947606 & -114.962842 & -114.960638 & -114.959684 & -114.962547 \\
\hline 2.7 & 2.75 & -114.955456 & -114.970665 & -114.968288 & -114.967362 & -114.970352 \\
\hline 2.7 & 3.0 & -114.961941 & -114.976702 & -114.974219 & -114.973355 & -114.976312 \\
\hline 2.7 & 3.5 & -114.969062 & -114.982836 & -114.980173 & -114.979426 & -114.982291 \\
\hline 2.7 & 4.0 & -114.969597 & -114.982917 & -114.979921 & -114.979242 & -114.982219 \\
\hline 2.7 & 5.0 & -114.962493 & -114.976476 & -114.972075 & -114.971428 & -114.975135 \\
\hline 2.7 & 6.0 & -114.955953 & -114.971751 & -114.965233 & -114.964542 & -114.969090 \\
\hline 2.7 & 8.0 & -114.951454 & -114.970595 & -114.960515 & -114.959734 & -114.965209 \\
\hline 2.9 & 1.2 & -114.707378 & -114.715787 & -114.714738 & -114.714289 & -114.714178 \\
\hline 2.9 & 1.4 & -114.855317 & -114.864488 & -114.863337 & -114.862828 & -114.862543 \\
\hline 2.9 & 1.6 & -114.914213 & -114.924338 & -114.923059 & -114.922477 & -114.922595 \\
\hline 2.9 & 1.7325 & -114.929678 & -114.940572 & -114.939187 & -114.938549 & -114.939024 \\
\hline 2.9 & 1.8 & -114.933655 & -114.944990 & -114.943543 & -114.942873 & -114.943561 \\
\hline 2.9 & 2.0 & -114.937700 & -114.950522 & -114.948848 & -114.948072 & -114.949540 \\
\hline 2.9 & 2.25 & -114.938175 & -114.952933 & -114.950895 & -114.949979 & -114.952433 \\
\hline 2.9 & 2.5 & -114.940514 & -114.956550 & -114.954150 & -114.953144 & -114.956239 \\
\hline 2.9 & 2.75 & -114.944657 & -114.960994 & -114.958324 & -114.957322 & -114.960674 \\
\hline 2.9 & 3.0 & -114.948852 & -114.964858 & -114.962012 & -114.961070 & -114.964448 \\
\hline
\end{tabular}


Table S5. (continued)

\begin{tabular}{|c|c|c|c|c|c|c|}
\hline$R_{\mathrm{Be}-\mathrm{F}}$ & $R_{\mathrm{H}-\mathrm{F}}$ & CCSD & $\operatorname{CCSD}(\mathrm{T})$ & R-CCSD $(\mathrm{T})$ & CR-CCSD(T) & MRCI(Q) \\
\hline 2.9 & 3.5 & -114.954021 & -114.968959 & -114.965864 & -114.965063 & -114.968359 \\
\hline 2.9 & 4.0 & -114.954442 & -114.968777 & -114.965326 & -114.964612 & -114.968000 \\
\hline 2.9 & 5.0 & -114.948628 & -114.963431 & -114.958579 & -114.957918 & -114.961993 \\
\hline 2.9 & 6.0 & -114.943103 & -114.959525 & -114.952666 & -114.951984 & -114.956847 \\
\hline 2.9 & 8.0 & -114.939147 & -114.958543 & -114.948429 & -114.947704 & -114.953444 \\
\hline 3.1 & 1.2 & -114.719897 & -114.728243 & -114.727186 & -114.726742 & -114.726489 \\
\hline 3.1 & 1.4 & -114.866357 & -114.875452 & -114.874290 & -114.873786 & -114.873628 \\
\hline 3.1 & 1.6 & -114.922968 & -114.932989 & -114.931697 & -114.931123 & -114.931142 \\
\hline 3.1 & 1.7325 & -114.936392 & -114.947157 & -114.945759 & -114.945131 & -114.945476 \\
\hline 3.1 & 1.8 & -114.939154 & -114.950345 & -114.948886 & -114.948228 & -114.948767 \\
\hline 3.1 & 2.0 & -114.938920 & -114.951587 & -114.949901 & -114.949142 & -114.950427 \\
\hline 3.1 & 2.25 & -114.933172 & -114.947974 & -114.945902 & -114.944995 & -114.947386 \\
\hline 3.1 & 2.5 & -114.929818 & -114.946396 & -114.943873 & -114.942840 & -114.946049 \\
\hline 3.1 & 2.75 & -114.929882 & -114.947232 & -114.944320 & -114.943249 & -114.946891 \\
\hline 3.1 & 3.0 & -114.931519 & -114.948769 & -114.945582 & -114.944560 & -114.948331 \\
\hline 3.1 & 3.5 & -114.934411 & -114.950591 & -114.947049 & -114.946187 & -114.949932 \\
\hline 3.1 & 4.0 & -114.934498 & -114.949919 & -114.945989 & -114.945238 & -114.949063 \\
\hline 3.1 & 5.0 & -114.929685 & -114.945356 & -114.940042 & -114.939369 & -114.943824 \\
\hline 3.1 & 6.0 & -114.925027 & -114.942154 & -114.934933 & -114.934261 & -114.939441 \\
\hline 3.1 & 8.0 & -114.921542 & -114.941402 & -114.931147 & -114.930465 & -114.936462 \\
\hline 3.3 & 1.2 & -114.729225 & -114.737455 & -114.736406 & -114.735968 & -114.735572 \\
\hline 3.3 & 1.4 & -114.874485 & -114.883443 & -114.882289 & -114.881794 & -114.881378 \\
\hline 3.3 & 1.6 & -114.929251 & -114.939102 & -114.937818 & -114. & -114 \\
\hline 3.3 & 1.7325 & -114.941025 & -114.951584 & -114.950197 & -114.949583 & -114.949795 \\
\hline 3.3 & 1.8 & -114.942796 & -114.953761 & -114.952313 & -114.951671 & -114.952059 \\
\hline 3.3 & 2.0 & -114.938991 & -114.951366 & -114.949701 & -114.948966 & -114.950026 \\
\hline 3.3 & 2.25 & -114.927633 & -114.942197 & -114.940150 & -114.939274 & -114.941483 \\
\hline 3.3 & 2.5 & -114.918505 & -114.935267 & -114.932713 & -114.931689 & -114.934873 \\
\hline 3.3 & 2.75 & -114.914059 & -114.932190 & -114.929119 & -114.928006 & -114.931816 \\
\hline 3.3 & 3.0 & -114.912797 & -114.931226 & -114.927749 & -114.926648 & -114.930741 \\
\hline 3.3 & 3.5 & -114.913080 & -114.930568 & -114.926583 & -114.925651 & -114.929835 \\
\hline 3.3 & 4.0 & -114.912646 & -114.929224 & -114.924802 & -114.924010 & -114.928281 \\
\hline 3.3 & 5.0 & -114.908575 & -114.925169 & -114.919382 & -114.918699 & -114.923539 \\
\hline 3.3 & 6.0 & -114.904646 & -114.922564 & -114.914954 & -114.914292 & -114.919789 \\
\hline 3.3 & 8.0 & -114.901569 & -114.922084 & -114.911588 & -114.910939 & -114.917183 \\
\hline 3.5 & 1.2 & -114.736723 & -114.744781 & -114.743755 & -114.743325 & -114.742596 \\
\hline 3.5 & 1.4 & -114.881029 & -114.889794 & -114.888663 & -114.888178 & -114.887699 \\
\hline 3.5 & 1.6 & -114.934331 & -114.943949 & -114.942691 & -114.942142 & -114.941975 \\
\hline 3.5 & 1.7325 & -114.944795 & -114.955081 & -114.953723 & -114.953127 & -114.953219 \\
\hline 3.5 & 1.8 & -114.945777 & -114.956444 & -114.955028 & -114.954405 & -114.954653 \\
\hline 3.5 & 2.0 & -114.939094 & -114.951076 & -114.949456 & -114.948748 & -114.949577 \\
\hline 3.5 & 2.25 & -114.922956 & -114.937051 & -114.935074 & -114.934238 & -114.936157 \\
\hline 3.5 & 2.5 & -114.908290 & -114.924822 & -114.922332 & -114.921349 & -114.924388 \\
\hline 3.5 & 2.75 & -114.898936 & -114.917464 & -114.914353 & -114.913240 & -114.917047 \\
\hline 3.5 & 3.0 & -114.894313 & -114.913739 & -114.910060 & -114.908901 & -114.913196 \\
\hline 3.5 & 3.5 & -114.891564 & -114.910418 & -114.906007 & -114.904994 & -114.909579 \\
\hline 3.5 & 4.0 & -114.890446 & -114.908259 & -114.903339 & -114.902498 & -114.907207 \\
\hline 3.5 & 5.0 & -114.886902 & -114.904480 & -114.898207 & -114.897516 & -114.902740 \\
\hline 3.5 & 6.0 & -114.883584 & -114.902380 & -114.894351 & -114.893699 & -114.899509 \\
\hline
\end{tabular}


Table S5. (continued)

\begin{tabular}{|c|c|c|c|c|c|c|}
\hline$R_{\mathrm{Be}-\mathrm{F}}$ & $R_{\mathrm{H}-\mathrm{F}}$ & CCSD & $\operatorname{CCSD}(\mathrm{T})$ & $\mathrm{R}-\mathrm{CCSD}(\mathrm{T})$ & CR-CCSD(T) & MRCI(Q) \\
\hline 3.5 & 8.0 & -114.880860 & -114.902198 & -114.891374 & -114.890749 & -114.897232 \\
\hline 3.7 & 1.2 & -114.743020 & -114.750862 & -114.749867 & -114.749449 & -114.749395 \\
\hline 3.7 & 1.4 & -114.886588 & -114.895111 & -114.894014 & -114.893542 & -114.893738 \\
\hline 3.7 & 1.6 & -114.938749 & -114.948085 & -114.946864 & -114.946332 & -114.946086 \\
\hline 3.7 & 1.7325 & -114.948193 & -114.958156 & -114.956840 & -114.956263 & -114.956251 \\
\hline 3.7 & 1.8 & -114.948563 & -114.958879 & -114.957508 & -114.956908 & -114.957031 \\
\hline 3.7 & 2.0 & -114.939635 & -114.951162 & -114.949601 & -114.948923 & -114.949545 \\
\hline 3.7 & 2.25 & -114.919652 & -114.933134 & -114.931249 & -114.930458 & -114.932024 \\
\hline 3.7 & 2.5 & -114.900075 & -114.916015 & -114.913654 & -114.912730 & -114.915539 \\
\hline 3.7 & 2.75 & -114.885655 & -114.904073 & -114.901056 & -114.899990 & -114.903605 \\
\hline 3.7 & 3.0 & -114.877113 & -114.897178 & -114.893433 & -114.892263 & -114.896576 \\
\hline 3.7 & 3.5 & -114.870702 & -114.890954 & -114.886148 & -114.885047 & -114.889969 \\
\hline 3.7 & 4.0 & -114.868733 & -114.887882 & -114.882453 & -114.881550 & -114.886676 \\
\hline 3.7 & 5.0 & -114.865552 & -114.884193 & -114.877410 & -114.876709 & -114.882312 \\
\hline 3.7 & 6.0 & -114.862745 & -114.882518 & -114.874030 & -114.873387 & -114.879503 \\
\hline 3.7 & 8.0 & -114.860327 & -114.882653 & -114.871413 & -114.870806 & -114.877516 \\
\hline 3.9 & 1.2 & -114.748399 & -114.755996 & -114.755037 & -114.754630 & -114.754397 \\
\hline 3.9 & 1.4 & -114.891407 & -114.899658 & -114.898600 & -114.898143 & -114.898129 \\
\hline 3.9 & 1.6 & -114.942698 & -114.9 & -114.950544 & -114.950029 & -114 \\
\hline 3.9 & 1.7325 & -114.951363 & -114.960973 & -114.959706 & -114.959150 & -114.959048 \\
\hline 3.9 & 1.8 & -114.951267 & -114.961204 & -114.959885 & -114.959307 & -114.959326 \\
\hline 3.9 & 2.0 & -114.940633 & -114.951678 & -114.950183 & -114.949535 & -114.949985 \\
\hline 3.9 & 2.25 & -114.917701 & -114.930516 & -114.928729 & -114.927982 & -114.929211 \\
\hline 3.9 & 2.5 & -114.894124 & -114.909249 & -114.907042 & -114.906179 & -114.908635 \\
\hline 3.9 & 2.75 & -114.874947 & -114.892752 & -114.889930 & -114.888940 & -114.892245 \\
\hline 3.9 & 3.0 & -114.862008 & -114.882165 & -114.878528 & -114.877409 & -114.881470 \\
\hline 3.9 & 3.5 & -114.850978 & -114 & -114.867451 & -114 & -114 \\
\hline 3.9 & 4.0 & -114.847940 & -114.868560 & -114.862597 & -114.861616 & -114.867128 \\
\hline 3.9 & 5.0 & -114.845003 & -114.864821 & -114.857485 & -114.856768 & -114.862745 \\
\hline 3.9 & 6.0 & -114.842633 & -114.863506 & -114.854500 & -114.853864 & -114.860281 \\
\hline 3.9 & 8.0 & -114.840484 & -114.863969 & -114.852219 & -114.851624 & -114.858550 \\
\hline 4.1 & 1.2 & -114.752984 & -114.760327 & -114.759405 & -114.759011 & -114.758612 \\
\hline 4.1 & 1.4 & -114.895578 & -114.903546 & -114.902529 & -114.902087 & -114.901897 \\
\hline 4.1 & 1.6 & -114.946216 & -114.954915 & -114.953784 & -114.953288 & -114.953365 \\
\hline 4.1 & 1.7325 & -114.954297 & -114.963547 & -114.962330 & -114.961795 & -114.961617 \\
\hline 4.1 & 1.8 & -114.953850 & -114.963404 & -114.962139 & -114.961583 & -114.961515 \\
\hline 4.1 & 2.0 & -114.941944 & -114.952514 & -114.951085 & -114.950465 & -114.950777 \\
\hline 4.1 & 2.25 & -114.916826 & -114.928986 & -114.927292 & -114.926585 & -114.927539 \\
\hline 4.1 & 2.5 & -114.890219 & -114.904465 & -114.902403 & -114.901595 & -114.903571 \\
\hline 4.1 & 2.75 & -114.867067 & -114.883928 & -114.881331 & -114.880415 & -114.883524 \\
\hline 4.1 & 3.0 & -114.849678 & -114.869310 & -114.865936 & -114.864908 & -114.868388 \\
\hline 4.1 & 3.5 & -114.832747 & -114.855514 & -114.850176 & -114.848967 & -114.854218 \\
\hline 4.1 & 4.0 & -114.828271 & -114.850533 & -114.843999 & -114.842923 & -114.848785 \\
\hline 4.1 & 5.0 & -114.825501 & -114.846660 & -114.838696 & -114.837953 & -114.844303 \\
\hline 4.1 & 6.0 & -114.823516 & -114.845651 & -114.836045 & -114.835410 & -114.842126 \\
\hline 4.1 & 8.0 & -114.821606 & -114.846452 & -114.834078 & -114.833491 & -114.840625 \\
\hline 4.5 & 1.2 & -114.760047 & -114.766913 & -114.766056 & -114.765686 & -114.763902 \\
\hline 4.5 & 1.4 & -114.902123 & -114.909559 & -114.908615 & -114.908202 & -114.907725 \\
\hline 4.5 & 1.6 & -114.951925 & -114.960019 & -114.958973 & -114.958510 & -114.958261 \\
\hline
\end{tabular}


Table S5. (continued)

\begin{tabular}{|c|c|c|c|c|c|c|}
\hline$R_{\mathrm{Be}-\mathrm{F}}$ & $R_{\mathrm{H}-\mathrm{F}}$ & CCSD & $\operatorname{CCSD}(\mathrm{T})$ & R-CCSD $(\mathrm{T})$ & CR-CCSD(T) & $\operatorname{MRCI}(\mathrm{Q})$ \\
\hline 4.5 & 1.7325 & -114.959254 & -114.967835 & -114.966711 & -114.966214 & -114.966141 \\
\hline 4.5 & 1.8 & -114.958357 & -114.967204 & -114.966037 & -114.965523 & -114.965546 \\
\hline 4.5 & 2.0 & -114.944828 & -114.954543 & -114.953232 & -114.952664 & -114.953018 \\
\hline 4.5 & 2.25 & -114.916981 & -114.928001 & -114.926464 & -114.925825 & -114.926427 \\
\hline 4.5 & 2.5 & -114.886655 & -114.899337 & -114.897501 & -114.896782 & -114.898047 \\
\hline 4.5 & 2.75 & -114.858453 & -114.873298 & -114.871047 & -114.870238 & -114.872491 \\
\hline 4.5 & 3.0 & -114.834343 & -114.851896 & -114.849066 & -114.848173 & -114.850999 \\
\hline 4.5 & 3.5 & -114.802707 & -114.826039 & -114.821157 & -114.820137 & -114.824282 \\
\hline 4.5 & 4.0 & -114.792605 & -114.818523 & -114.810876 & -114.809656 & -114.816040 \\
\hline 4.5 & 5.0 & -114.789950 & -114.814659 & -114.805015 & -114.804154 & -114.811245 \\
\hline 4.5 & 6.0 & -114.788700 & -114.814123 & -114.802908 & -114.802241 & -114.809562 \\
\hline 4.5 & 8.0 & -114.787212 & -114.815623 & -114.801537 & -114.800943 & -114.808474 \\
\hline 4.7 & 1.2 & -114.762653 & -114.769313 & -114.768483 & -114.768125 & -114.766338 \\
\hline 4.7 & 1.4 & -114.904574 & -114.911781 & -114.910868 & -114.910467 & -114.909880 \\
\hline 4.7 & 1.6 & -114.954120 & -114.961955 & -114.960943 & -114.960495 & -114.960123 \\
\hline 4.7 & 1.7325 & -114.961216 & -114.969512 & -114.968426 & -114.967946 & -114.967740 \\
\hline 4.7 & 1.8 & -114.960180 & -114.968726 & -114.967599 & -114.967103 & -114.966987 \\
\hline 4.7 & 2.0 & -114.946152 & -114.955510 & -114.954247 & -114.953700 & -114 \\
\hline 4.7 & 2.25 & -114.917487 & -114.928045 & -114.926571 & -114.925959 & -114.926559 \\
\hline 4.7 & 2.5 & -114.886097 & -114.898159 & -114.896406 & -114.895721 & -114.896784 \\
\hline 4.7 & 2.75 & -114.856512 & -114.870522 & -114.868384 & -114.867614 & -114.869444 \\
\hline 4.7 & 3.0 & -114.830528 & -114.847049 & -114.844375 & -114 & -114.846450 \\
\hline 4.7 & 3.5 & -114.792457 & -114.815131 & -114.810728 & -114.809793 & -114.811864 \\
\hline 4.7 & 4.0 & -114.776835 & -114.804273 & -114.796450 & -114.795297 & -114.801668 \\
\hline 4.7 & 5.0 & -114.773843 & -114.801095 & -114.790232 & -114.789262 & -114.796708 \\
\hline 4.7 & 6.0 & -114.773019 & -114.800731 & -114.788376 & -114.787658 & -114 \\
\hline 4.7 & 8.0 & -114.771740 & -114.802594 & -114.787302 & -114.786681 & -114.794384 \\
\hline 5.0 & 1.2 & -114.765612 & -114.772014 & -114.771217 & -114.770871 & -114.769086 \\
\hline 5.0 & 1.4 & -114.907380 & -114.914299 & -114.913423 & -114.913037 & -114.911072 \\
\hline 5.0 & 1.6 & -114.956668 & -114 & -114.963207 & -114.962778 & -114.960653 \\
\hline 5.0 & 1.7325 & -114.963528 & -114.971466 & -114.970427 & -114.969968 & -114.969608 \\
\hline 5.0 & 1.8 & -114.962350 & -114.970520 & -114.969443 & -114.968969 & -114.968693 \\
\hline 5.0 & 2.0 & -114.947817 & -114.956733 & -114.955528 & -114.955008 & -114.955000 \\
\hline 5.0 & 2.25 & -114.918338 & -114.928337 & -114.926937 & -114.926358 & -114.926713 \\
\hline 5.0 & 2.5 & -114.885930 & -114.897258 & -114.895601 & -114.894957 & -114.895687 \\
\hline 5.0 & 2.75 & -114.855108 & -114.868137 & -114.866122 & -114.865399 & -114.866466 \\
\hline 5.0 & 3.0 & -114.827587 & -114.842856 & -114.840326 & -114.839500 & -114.840740 \\
\hline 5.0 & 3.5 & -114.784080 & -114.805770 & -114.801528 & -114.800481 & -114.801121 \\
\hline 5.0 & 4.0 & -114.757675 & -114.786122 & -114.779084 & -114.778162 & -114.782583 \\
\hline 5.0 & 5.0 & -114.751461 & -114.784380 & -114.770791 & -114.769577 & -114.777427 \\
\hline 5.0 & 6.0 & -114.751522 & -114.784273 & -114.769396 & -114.768496 & -114.776435 \\
\hline 5.0 & 8.0 & -114.750626 & -114.786712 & -114.768824 & -114.768070 & -114.775891 \\
\hline 5.2 & 1.2 & -114.767063 & -114.773325 & -114.772546 & -114.772208 & -114.770424 \\
\hline 5.2 & 1.4 & -114.908765 & -114.915527 & -114.914671 & -114.914294 & -114.912332 \\
\hline 5.2 & 1.6 & -114.957939 & -114.965270 & -114.964324 & -114.963904 & -114.961784 \\
\hline 5.2 & 1.7325 & -114.964694 & -114.972438 & -114.971424 & -114.970976 & -114.970536 \\
\hline 5.2 & 1.8 & -114.963453 & -114.971419 & -114.970368 & -114.969906 & -114.969548 \\
\hline 5.2 & 2.0 & -114.948694 & -114.957372 & -114.956199 & -114.955692 & -114.955594 \\
\hline 5.2 & 2.25 & -114.918858 & -114.928561 & -114.927199 & -114.926638 & -114.926892 \\
\hline
\end{tabular}


Table S5. (continued)

\begin{tabular}{|c|c|c|c|c|c|c|}
\hline$R_{\mathrm{Be}-\mathrm{F}}$ & $R_{\mathrm{H}-\mathrm{F}}$ & CCSD & $\operatorname{CCSD}(\mathrm{T})$ & $\mathrm{R}-\mathrm{CCSD}(\mathrm{T})$ & CR-CCSD $(\mathrm{T})$ & $\operatorname{MRCI}(\mathrm{Q})$ \\
\hline 5.2 & 2.5 & -114.886021 & -114.896969 & -114.895360 & -114.894738 & -114.895353 \\
\hline 5.2 & 2.75 & -114.854713 & -114.867244 & -114.865291 & -114.864593 & -114.865534 \\
\hline 5.2 & 3.0 & -114.826664 & -114.841292 & -114.838830 & -114.838026 & -114.839168 \\
\hline 5.2 & 3.5 & -114.781775 & -114.802945 & -114.798572 & -114.797422 & -114.798224 \\
\hline 5.2 & 4.0 & -114.750784 & -114.780749 & -114.773334 & -114.772012 & -114.772889 \\
\hline 5.2 & 5.0 & -114.737522 & -114.776288 & -114.759929 & -114.758514 & -114.766310 \\
\hline 5.2 & 6.0 & -114.738467 & -114.776650 & -114.759045 & -114.757862 & -114.765753 \\
\hline 5.2 & 8.0 & -114.737960 & -114.779517 & -114.758905 & -114.757897 & -114.765522 \\
\hline 5.5 & 1.2 & -114.768641 & -114.774734 & -114.773975 & -114.773646 & -114.771867 \\
\hline 5.5 & 1.4 & -114.910275 & -114.916849 & -114.916016 & -114.915649 & -114.913694 \\
\hline 5.5 & 1.6 & -114.959334 & -114.966450 & -114.965531 & -114.965124 & -114.963013 \\
\hline 5.5 & 1.7325 & -114.965980 & -114.973491 & -114.972507 & -114.972072 & -114.969882 \\
\hline 5.5 & 1.8 & -114.964675 & -114.972396 & -114.971377 & -114.970928 & -114.970281 \\
\hline 5.5 & 2.0 & -114.949686 & -114.958079 & -114.956942 & -114.956453 & -114.956251 \\
\hline 5.5 & 2.25 & -114.919490 & -114.928844 & -114.927527 & -114.926986 & -114.927126 \\
\hline 5.5 & 2.5 & -114.886236 & -114.896742 & -114.895190 & -114.894592 & -114.895090 \\
\hline 5.5 & 2.75 & -114.854487 & -114.866449 & -114.864567 & -114.863896 & -114.864737 \\
\hline 5.5 & 3.0 & -114.826020 & -114.839914 & -114.837535 & -114.836759 & -114.837499 \\
\hline 5.5 & 3.5 & -114.780522 & -114.800884 & -114.796407 & -114.795192 & -114.796480 \\
\hline 5.5 & 4.0 & -114.748633 & -114.781850 & -114.771898 & -114.769557 & -114.772046 \\
\hline 5.5 & 5.0 & -114.718475 & -114.772862 & -114.749820 & -114.747683 & -114.754743 \\
\hline 5.5 & 6.0 & -114.721013 & -114.775460 & -114.749347 & -114.746958 & -114.757461 \\
\hline 5.5 & 8.0 & -114.721656 & -114.778800 & -114.750083 & -114.747855 & -114.757824 \\
\hline 6.0 & 1.2 & -114.770153 & -114.776048 & -114.775313 & -114.774993 & -114.773223 \\
\hline 6.0 & 1.4 & -114.911721 & -114.918073 & -114.917267 & -114.916912 & -114.914968 \\
\hline 6.0 & 1.6 & -114.960668 & -114.967534 & -114.966646 & -114.966253 & -114.964156 \\
\hline 6.0 & 1.7325 & -114.967212 & -114.974449 & -114.973500 & -114.973080 & -114.970908 \\
\hline 6.0 & 1.8 & -114.965846 & -114.973281 & -114.972297 & -114.971865 & -114.971111 \\
\hline 6.0 & 2.0 & -114.950643 & -114.958705 & -114.957610 & -114.957140 & -114.956578 \\
\hline 6.0 & 2.25 & -114.920121 & -114.929072 & -114.927807 & -114.927289 & -114.927005 \\
\hline 6.0 & 2.5 & -114.886508 & -114.896517 & -114.895028 & -114.894458 & -114.894467 \\
\hline 6.0 & 2.75 & -114.854419 & -114.865754 & -114.863951 & -114.863311 & -114.863621 \\
\hline 6.0 & 3.0 & -114.825700 & -114.838791 & -114.836513 & -114.835769 & -114.836419 \\
\hline 6.0 & 3.5 & -114.780324 & -114.799398 & -114.795053 & -114.793850 & -114.795603 \\
\hline 6.0 & 4.0 & -114.749878 & -114.782505 & -114.771750 & -114.769090 & -114.772122 \\
\hline 6.0 & 5.0 & -114.720289 & -114.850909 & -114.774311 & -114.758068 & -114.756066 \\
\hline 6.0 & 6.0 & -114.707846 & -114.886328 & -114.775313 & -114.756781 & -114.755034 \\
\hline 6.0 & 8.0 & -114.709551 & -114.849650 & -114.765062 & -114.752892 & -114.755246 \\
\hline 8.0 & 1.2 & -114.770936 & -114.776538 & -114.775838 & -114.775534 & -114.773794 \\
\hline 8.0 & 1.4 & -114.912371 & -114.918393 & -114.917628 & -114.917290 & -114.915384 \\
\hline 8.0 & 1.6 & -114.961147 & -114.967639 & -114.966798 & -114.966425 & -114.964377 \\
\hline 8.0 & 1.7325 & -114.967558 & -114.974386 & -114.973487 & -114.973092 & -114.972253 \\
\hline 8.0 & 1.8 & -114.966117 & -114.973124 & -114.972194 & -114.971787 & -114.970990 \\
\hline 8.0 & 2.0 & -114.950677 & -114.958246 & -114.957214 & -114.956773 & -114.956126 \\
\hline 8.0 & 2.25 & -114.919842 & -114.928201 & -114.927012 & -114.926529 & -114.926116 \\
\hline 8.0 & 2.5 & -114.885944 & -114.895239 & -114.893844 & -114.893312 & -114.893177 \\
\hline 8.0 & 2.75 & -114.853667 & -114.864134 & -114.862447 & -114.861849 & -114.862047 \\
\hline 8.0 & 3.0 & -114.824941 & -114.836954 & -114.834831 & -114.834129 & -114.834775 \\
\hline 8.0 & 3.5 & -114.780456 & -114.797445 & -114.793592 & -114.792456 & -114.794582 \\
\hline
\end{tabular}


Table S5. (continued)

\begin{tabular}{lcccccc}
\hline$R_{\mathrm{Be}-\mathrm{F}}$ & $R_{\mathrm{H}-\mathrm{F}}$ & $\mathrm{CCSD}$ & $\mathrm{CCSD}(\mathrm{T})$ & $\mathrm{R}-\mathrm{CCSD}(\mathrm{T})$ & $\mathrm{CR}-\mathrm{CCSD}(\mathrm{T})$ & $\mathrm{MRCI}(\mathrm{Q})$ \\
\hline 8.0 & 4.0 & -114.752296 & -114.778255 & -114.770372 & -114.768235 & -114.772158 \\
8.0 & 5.0 & -114.727825 & -114.790293 & -114.759935 & -114.752842 & -114.757089 \\
8.0 & 6.0 & -114.721230 & -114.846415 & -114.769678 & -114.753791 & -114.754564 \\
8.0 & 8.0 & -114.718237 & -114.943962 & -114.786832 & -114.759066 & -114.754029 \\
\hline
\end{tabular}

Table S6. The CCSD, CCSD $(\mathrm{T}), \mathrm{R}-\mathrm{CCSD}(\mathrm{T}), \mathrm{CR}-\mathrm{CCSD}(\mathrm{T})$, and $\mathrm{MRCI}(\mathrm{Q})$ energies, in hartree, of the BeFH system, as described by the cc-pVTZ basis set, for the $\mathrm{Be}-\mathrm{F}-\mathrm{H}$ angle $\theta=45^{\circ}$. The $\mathrm{Be}-\mathrm{F}$ and $\mathrm{H}-\mathrm{F}$ bond distances, $R_{\mathrm{Be}-\mathrm{F}}$ and $R_{\mathrm{H}-\mathrm{F}}$, respectively, are in bohr.

\begin{tabular}{|c|c|c|c|c|c|c|}
\hline$R_{\mathrm{Be}-\mathrm{F}}$ & $R_{\mathrm{H}-\mathrm{F}}$ & CCSD & $\operatorname{CCSD}(\mathrm{T})$ & R-CCSD $(\mathrm{T})$ & CR-CCSD(T) & $\operatorname{MRCI}(\mathrm{Q})$ \\
\hline 1.8 & 1.2 & -113.998445 & -114.006754 & -114.005998 & -114.005543 & -114.005381 \\
\hline 1.8 & 1.4 & -114.137214 & -114.146385 & -114.145549 & -114.145028 & -114.145264 \\
\hline 1.8 & 1.6 & -114.233152 & -114.243200 & -114.242291 & -114.241699 & -114.242418 \\
\hline 1.8 & 1.7325 & -114.295450 & -114.305870 & -114.304938 & -114.304312 & -114.305290 \\
\hline 1.8 & 1.8 & -114.327999 & -114.338501 & -114.337570 & -114.336934 & -114.338007 \\
\hline 1.8 & 2.0 & -114.424860 & -114.435220 & -114.434332 & -114.433695 & -114.434880 \\
\hline 1.8 & 2.25 & -114.535425 & -114.545207 & -114.544406 & -114.543800 & -114.544905 \\
\hline 1.8 & 2.5 & -114.623199 & -114.632386 & -114.631663 & -114.631092 & -114.632089 \\
\hline 1.8 & 2.75 & -114.685740 & -114.694461 & -114.693790 & -114.693249 & -114.694171 \\
\hline 1.8 & 3.0 & -114.726212 & -114.734594 & -114.733956 & -114.733436 & -114.734317 \\
\hline 1.8 & 3.5 & -114.760272 & -114.768252 & -114.767632 & -114.767141 & -114.768003 \\
\hline 1.8 & 4.0 & -114.760548 & -114.768369 & -114.767715 & -114.767242 & -114.768147 \\
\hline 1.8 & 5.0 & -114.728220 & -114.736288 & -114.735356 & -114.734902 & -114.736109 \\
\hline 1.8 & 6.0 & -114.694882 & -114.704138 & -114.702314 & -114.701855 & -114.703813 \\
\hline 1.8 & 8.0 & -114.667377 & -114.683234 & -114.676031 & -114.675283 & -114.679001 \\
\hline 1.9 & 1.2 & -114.152647 & -114.161070 & -114.160271 & -114.159810 & -114.159630 \\
\hline 1.9 & 1.4 & -114.285471 & -114.294790 & -114.293903 & -114.293374 & -114.293624 \\
\hline 1.9 & 1.6 & -114.367891 & -114.378177 & -114.377203 & -114.376597 & -114.377389 \\
\hline 1.9 & 1.7325 & -114.419675 & -114.430421 & -114.429416 & -114.428769 & -114.429847 \\
\hline 1.9 & 1.8 & -114.447048 & -114.457919 & -114.456911 & -114.456251 & -114.457436 \\
\hline 1.9 & 2.0 & -114.530931 & -114.541732 & -114.540763 & -114.540097 & -114.541421 \\
\hline 1.9 & 2.25 & -114.631555 & -114.641756 & -114.640885 & -114.640251 & -114.641490 \\
\hline 1.9 & 2.5 & -114.715245 & -114.724789 & -114.724010 & -114.723414 & -114.724521 \\
\hline 1.9 & 2.75 & -114.777207 & -114.786230 & -114.785516 & -114.784952 & -114.785966 \\
\hline 1.9 & 3.0 & -114.818752 & -114.827400 & -114.826725 & -114.826185 & -114.827146 \\
\hline 1.9 & 3.5 & -114.856015 & -114.864221 & -114.863571 & -114.863062 & -114.863992 \\
\hline 1.9 & 4.0 & -114.858424 & -114.866447 & -114.865769 & -114.865279 & -114.866245 \\
\hline 1.9 & 5.0 & -114.827673 & -114.835901 & -114.834953 & -114.834485 & -114.835742 \\
\hline 1.9 & 6.0 & -114.794258 & -114.803591 & -114.801775 & -114.801309 & -114.803309 \\
\hline 1.9 & 8.0 & -114.766189 & -114.781704 & -114.774730 & -114.774011 & -114.777841 \\
\hline 2.0 & 1.2 & -114.271860 & -114.280387 & -114.279543 & -114.279078 & -114.278884 \\
\hline 2.0 & 1.4 & -114.401808 & -114.411262 & -114.410323 & -114.409787 & -114.410072 \\
\hline 2.0 & 1.6 & -114.474296 & -114.484797 & -114.483758 & -114.483139 & -114.484000 \\
\hline 2.0 & 1.7325 & -114.517361 & -114.528413 & -114.527332 & -114.526666 & -114.527872 \\
\hline 2.0 & 1.8 & -114.540191 & -114.551415 & -114.550326 & -114.549643 & -114.550977 \\
\hline
\end{tabular}


Table S6. (continued)

\begin{tabular}{|c|c|c|c|c|c|c|}
\hline$R_{\mathrm{Be}-\mathrm{F}}$ & $R_{\mathrm{H}-\mathrm{F}}$ & CCSD & $\operatorname{CCSD}(\mathrm{T})$ & $\mathrm{R}-\mathrm{CCSD}(\mathrm{T})$ & CR-CCSD $(\mathrm{T})$ & $\operatorname{MRCI}(\mathrm{Q})$ \\
\hline 2.0 & 2.0 & -114.611910 & -114.623157 & -114.622101 & -114.621404 & -114.622899 \\
\hline 2.0 & 2.25 & -114.702123 & -114.712763 & -114.711814 & -114.711149 & -114.712544 \\
\hline 2.0 & 2.5 & -114.780708 & -114.790627 & -114.789786 & -114.789164 & -114.790395 \\
\hline 2.0 & 2.75 & -114.841164 & -114.850503 & -114.849740 & -114.849153 & -114.850270 \\
\hline 2.0 & 3.0 & -114.883125 & -114.892049 & -114.891333 & -114.890772 & -114.891823 \\
\hline 2.0 & 3.5 & -114.922979 & -114.931416 & -114.930736 & -114.930208 & -114.931214 \\
\hline 2.0 & 4.0 & -114.927441 & -114.935672 & -114.934968 & -114.934460 & -114.935496 \\
\hline 2.0 & 5.0 & -114.898355 & -114.906753 & -114.905787 & -114.905303 & -114.906622 \\
\hline 2.0 & 6.0 & -114.864946 & -114.874381 & -114.872565 & -114.872090 & -114.874147 \\
\hline 2.0 & 8.0 & -114.836354 & -114.851601 & -114.844817 & -114.844126 & -114.848077 \\
\hline 2.2 & 1.2 & -114.435948 & -114.444648 & -114.443718 & -114.443245 & -114.443019 \\
\hline 2.2 & 1.4 & -114.565380 & -114.575057 & -114.574016 & -114.573469 & -114.573693 \\
\hline 2.2 & 1.6 & -114.625920 & -114.636779 & -114.635613 & -114.634975 & -114.635847 \\
\hline 2.2 & 1.7325 & -114.656351 & -114.667935 & -114.666702 & -114.666003 & -114.667313 \\
\hline 2.2 & 1.8 & -114.672050 & -114.683911 & -114.682657 & -114.681934 & -114.683421 \\
\hline 2.2 & 2.0 & -114.722906 & -114.735039 & -114.733794 & -114.733039 & -114.734783 \\
\hline 2.2 & 2.25 & -114.792578 & -114.804150 & -114.803022 & -114.802296 & -114.803962 \\
\hline 2.2 & 2.5 & -114.859015 & -114.869746 & -114.868760 & -114.868083 & -114.869557 \\
\hline 2.2 & 2.75 & -114.914169 & -114.924188 & -114.923311 & -114.922677 & -114.924008 \\
\hline 2.2 & 3.0 & -114.955113 & -114.964618 & -114.963811 & -114.963208 & -114.964454 \\
\hline 2.2 & 3.5 & -114.998171 & -115.007086 & -115.006338 & -115.005772 & -115.006949 \\
\hline 2.2 & 4.0 & -115.006328 & -115.014989 & -115.014227 & -115.013684 & -115.0 \\
\hline 2.2 & 5.0 & -114.980766 & -114.989530 & -114.988519 & -114.988003 & -114.989444 \\
\hline 2.2 & 6.0 & -114.947632 & -114.957324 & -114.955490 & -114.954995 & -114.957169 \\
\hline 2.2 & 8.0 & -114.918125 & -114.933028 & -114.926527 & -114.925881 & -114.930066 \\
\hline 2.4 & 1.2 & -114.535992 & 14815 & -114.543809 & -114.543332 & 43016 \\
\hline 2.4 & 1.4 & -114.667779 & -114.677612 & -114.676481 & -114.675928 & -114.676067 \\
\hline 2.4 & 1.6 & -114.722918 & -114.734029 & -114.732748 & -114.732098 & -114.732937 \\
\hline 2.4 & 1.7325 & -114.745599 & -114.757587 & -114.756211 & -114.755490 & -114.756844 \\
\hline 2.4 & 1.8 & -114.756430 & -114.768801 & -114.767388 & -114.766635 & -114.768222 \\
\hline 2.4 & 2.0 & -114.791353 & -114.804320 & -114.802872 & -114.802064 & -114.804053 \\
\hline 2.4 & 2.25 & -114.842610 & -114.855180 & -114.853838 & -114.853049 & -114.855002 \\
\hline 2.4 & 2.5 & -114.895946 & -114.907583 & -114.906415 & -114.905684 & -114.907392 \\
\hline 2.4 & 2.75 & -114.943696 & -114.954469 & -114.953452 & -114.952772 & -114.954280 \\
\hline 2.4 & 3.0 & -114.981543 & -114.991682 & -114.990765 & -114.990121 & -114.991509 \\
\hline 2.4 & 3.5 & -115.025182 & -115.034595 & -115.033767 & -115.033166 & -115.034456 \\
\hline 2.4 & 4.0 & -115.036274 & -115.045377 & -115.044550 & -115.043972 & -115.045261 \\
\hline 2.4 & 5.0 & -115.014371 & -115.023521 & -115.022457 & -115.021909 & -115.023438 \\
\hline 2.4 & 6.0 & -114.981857 & -114.991858 & -114.989989 & -114.989470 & -114.991731 \\
\hline 2.4 & 8.0 & -114.951624 & -114.966396 & -114.960076 & -114.959462 & -114.963842 \\
\hline 2.5 & 1.2 & -114.570878 & -114.579744 & -114.578705 & -114.578227 & -114.577866 \\
\hline 2.5 & 1.4 & -114.704068 & -114.713953 & -114.712783 & -114.712229 & -114.712320 \\
\hline 2.5 & 1.6 & -114.757855 & -114.769052 & -114.767720 & -114.767068 & -114.767870 \\
\hline 2.5 & 1.7325 & -114.777919 & -114.790054 & -114.788615 & -114.787888 & -114.789241 \\
\hline 2.5 & 1.8 & -114.786969 & -114.799536 & -114.798050 & -114.797288 & -114.798896 \\
\hline 2.5 & 2.0 & -114.815574 & -114.828917 & -114.827369 & -114.826539 & -114.828635 \\
\hline 2.5 & 2.25 & -114.858719 & -114.871804 & -114.870343 & -114.869524 & -114.871630 \\
\hline 2.5 & 2.5 & -114.905602 & -114.917732 & -114.916458 & -114.915699 & -114.917540 \\
\hline 2.5 & 2.75 & -114.949203 & -114.960389 & -114.959289 & -114.958586 & -114.960193 \\
\hline
\end{tabular}


Table S6. (continued)

\begin{tabular}{|c|c|c|c|c|c|c|}
\hline$R_{\mathrm{Be}-\mathrm{F}}$ & $R_{\mathrm{H}-\mathrm{F}}$ & CCSD & $\operatorname{CCSD}(\mathrm{T})$ & $\mathrm{R}-\mathrm{CCSD}(\mathrm{T})$ & CR-CCSD $(\mathrm{T})$ & MRCI(Q) \\
\hline 2.5 & 3.0 & -114.984909 & -114.995389 & -114.994408 & -114.993745 & -114.995210 \\
\hline 2.5 & 3.5 & -115.027930 & -115.037601 & -115.036729 & -115.036112 & -115.037459 \\
\hline 2.5 & 4.0 & -115.040149 & -115.049477 & -115.048614 & -115.048021 & -115.049361 \\
\hline 2.5 & 5.0 & -115.020078 & -115.029424 & -115.028330 & -115.027767 & -115.029344 \\
\hline 2.5 & 6.0 & -114.987999 & -114.998166 & -114.996275 & -114.995743 & -114.998052 \\
\hline 2.5 & 8.0 & -114.957475 & -114.972248 & -114.965986 & -114.965384 & -114.969861 \\
\hline 2.5719 & 1.2 & -114.591557 & -114.600444 & -114.599384 & -114.598906 & -114.598513 \\
\hline 2.5719 & 1.4 & -114.725720 & -114.735632 & -114.734437 & -114.733883 & -114.733943 \\
\hline 2.5719 & 1.6 & -114.778863 & -114.790105 & -114.788742 & -114.788089 & -114.788858 \\
\hline 2.5719 & 1.7325 & -114.797419 & -114.809636 & -114.808156 & -114.807427 & -114.808765 \\
\hline 2.5719 & 1.8 & -114.805396 & -114.818077 & -114.816544 & -114.815778 & -114.817392 \\
\hline 2.5719 & 2.0 & -114.830035 & -114.843625 & -114.842008 & -114.841164 & -114.843329 \\
\hline 2.5719 & 2.25 & -114.867816 & -114.881272 & -114.879723 & -114.878882 & -114.881100 \\
\hline 2.5719 & 2.5 & -114.910190 & -114.922693 & -114.921335 & -114.920556 & -114.922500 \\
\hline 2.5719 & 2.75 & 50714 & -114.962215 & 1048 & 60330 & 2012 \\
\hline 2.5719 & 3.0 & -114.984697 & -114.995435 & -114.994402 & -114.993726 & -114.995248 \\
\hline 2.5719 & 3.5 & -115.026938 & -115.036799 & -115.035893 & -115.035265 & -115.036655 \\
\hline 2.5719 & 4.0 & -115.039817 & -115. & -115 & -115 & -115 \\
\hline 2.5719 & 5.0 & -115.021046 & 0534 & 9416 & 8843 & -11 \\
\hline 2.5719 & 6.0 & -114.989328 & -114.999620 & -114.997710 & -114.997170 & -114.999515 \\
\hline 2.5719 & 8.0 & -114.958626 & -114.973422 & -114.967192 & -114.966598 & -114.971144 \\
\hline 2.6 & 1.2 & -114. & -114.607699 & -114.606632 & -114 & -114 \\
\hline 2.6 & 1.4 & -114.733332 & -114.743252 & -114.742049 & 41495 & 41542 \\
\hline 2.6 & 1.6 & -114.786280 & -114.797535 & -114.796160 & -114.795507 & -114.796262 \\
\hline 2.6 & 1.7325 & -114.804315 & -114.816559 & -114.815064 & -114.814334 & -114.815665 \\
\hline 2.6 & 1.8 & -114 & 4634 & -114 & -114 & -114 \\
\hline 2.6 & 2.0 & 35122 & -114.848802 & -114.847158 & 46309 & 348498 \\
\hline 2.6 & 2.25 & -114.870910 & -114.884510 & -114.882926 & -114.882077 & -114.884340 \\
\hline 2.6 & 2.5 & -114.911559 & -114.924212 & -114.922820 & -114.922033 & -114.924019 \\
\hline 2.6 & 2.75 & -114 & -114 & -114 & 0578 & 62292 \\
\hline 2.6 & 3.0 & -114.984143 & -114.994985 & -114.993931 & -114.993251 & -114.994796 \\
\hline 2.6 & 3.5 & -115.026009 & -115.035946 & -115.035026 & -115.034394 & -115.035800 \\
\hline 2.6 & 4.0 & -115.039111 & -115.048666 & -115.047764 & -115.047156 & -115.048549 \\
\hline 2.6 & 5.0 & -115.020842 & -115.030385 & -115.029259 & -115. & -115.030307 \\
\hline 2.6 & 6.0 & -114.989277 & -114.999618 & -114.997700 & -114.997157 & -114.999516 \\
\hline 2.6 & 8.0 & -114.958512 & -114.973322 & -114.967102 & -114.966511 & -114.971083 \\
\hline 2.7 & 1.2 & -114.621425 & -114.630333 & -114.629243 & -114.628766 & -114.628201 \\
\hline 2.7 & 1.4 & -114.757153 & -114.767091 & -114.765860 & -114.765307 & -114.765304 \\
\hline 2.7 & 1.6 & -114.809582 & -114.820870 & -114.819459 & -114.818807 & -114.819507 \\
\hline 2.7 & 1.7325 & -114.826030 & -114.838344 & -114.836803 & -114.836072 & -114.837363 \\
\hline 2.7 & 1.8 & -114.832442 & -114.845270 & -114.843665 & -114.842894 & -114.844486 \\
\hline 2.7 & 2.0 & -114.851039 & -114.865007 & -114.863276 & -114.862412 & -114.864672 \\
\hline 2.7 & 2.25 & -114.880194 & -114.894299 & -114.892591 & -114.891713 & -114.894134 \\
\hline 2.7 & 2.5 & -114.914902 & -114.928103 & -114.926583 & -114.925767 & -114.927910 \\
\hline 2.7 & 2.75 & -114.949868 & -114.961971 & -114.960671 & -114.959925 & -114.961757 \\
\hline 2.7 & 3.0 & -114.980493 & -114.991722 & -114.990588 & -114.989889 & -114.991522 \\
\hline 2.7 & 3.5 & -115.020729 & -115.030941 & -115.029970 & -115.029324 & -115.030789 \\
\hline 2.7 & 4.0 & -115.034464 & -115.044248 & -115.043305 & -115.042684 & -115.044129 \\
\hline 2.7 & 5.0 & -115.017945 & -115.027688 & -115.026527 & -115.025938 & -115.027611 \\
\hline
\end{tabular}


Table S6. (continued)

\begin{tabular}{|c|c|c|c|c|c|c|}
\hline$R_{\mathrm{Be}-\mathrm{F}}$ & $R_{\mathrm{H}-\mathrm{F}}$ & CCSD & $\operatorname{CCSD}(\mathrm{T})$ & R-CCSD $(\mathrm{T})$ & CR-CCSD(T) & $\operatorname{MRCI}(\mathrm{Q})$ \\
\hline 2.7 & 6.0 & -114.986971 & -114.997490 & -114.995544 & -114.994989 & -114.997400 \\
\hline 2.7 & 8.0 & -114.956016 & -114.970895 & -114.964703 & -114.964119 & -114.968788 \\
\hline 2.9 & 1.2 & -114.655435 & -114.664322 & -114.663206 & -114.662731 & -114.662758 \\
\hline 2.9 & 1.4 & -114.793053 & -114.802970 & -114.801705 & -114.801156 & -114.801084 \\
\hline 2.9 & 1.6 & -114.844925 & -114.856198 & -114.854742 & -114.854095 & -114.854672 \\
\hline 2.9 & 1.7325 & -114.859085 & -114.871428 & -114.869825 & -114.869100 & -114.870268 \\
\hline 2.9 & 1.8 & -114.863702 & -114.876611 & -114.874931 & -114.874164 & -114.875656 \\
\hline 2.9 & 2.0 & -114.874944 & -114.889312 & -114.887442 & -114.886561 & -114.888882 \\
\hline 2.9 & 2.25 & -114.892798 & -114.907826 & -114.905883 & -114.904953 & -114.907663 \\
\hline 2.9 & 2.5 & -114.916657 & -114.931017 & -114.929214 & -114.928340 & -114.930829 \\
\hline 2.9 & 2.75 & -114.943069 & -114.956234 & -114.954683 & -114.953892 & -114.955998 \\
\hline 2.9 & 3.0 & -114.968001 & -114.980099 & -114.978770 & -114.978039 & -114.979868 \\
\hline 2.9 & 3.5 & -115.003818 & -115.014625 & -115.013532 & -115.012864 & -115.014454 \\
\hline 2.9 & 4.0 & -115.018071 & -115.028331 & -115.027296 & -115.026654 & -115.028203 \\
\hline 2.9 & 5.0 & -115.004821 & -115.014966 & -115.013731 & -115.013120 & -115.014893 \\
\hline 2.9 & 6.0 & -114.975236 & -114.986126 & -114.984114 & -114.983539 & -114.986059 \\
\hline 2.9 & 8.0 & -114.944053 & -114.959157 & -114.952983 & -114.952410 & -114.957270 \\
\hline 3.1 & 1.2 & -114.679657 & -114.688455 & -114.687335 & -114.686866 & -114 \\
\hline 3.1 & 1.4 & -114.818501 & -114.828319 & -114.827047 & -114.826505 & -114 \\
\hline 3.1 & 1.6 & -114.870010 & -114.881163 & -114.879696 & -114.879060 & -114.879499 \\
\hline 3.1 & 1.7325 & -114.882582 & -114.894810 & -114.893187 & -114.892476 & -114.893481 \\
\hline 3.1 & 1.8 & -114.885913 & -114 & -114.897022 & 96268 & -114 \\
\hline 3.1 & 2.0 & -114.891614 & -114.906118 & -114.904169 & -114.903291 & -114.905560 \\
\hline 3.1 & 2.25 & -114.900359 & -114.916090 & -114.913959 & -114.912994 & -114.915910 \\
\hline 3.1 & 2.5 & -114.914833 & -114.930348 & -114.928257 & -114.927325 & -114.930173 \\
\hline 3.1 & 2.75 & -114.933186 & -114.947561 & -114 & -114.944871 & -114.947307 \\
\hline 3.1 & 3.0 & -114.952238 & -114.965362 & -114.963785 & -114.963024 & -114.965090 \\
\hline 3.1 & 3.5 & -114.982526 & -114.994010 & -114.992766 & -114.992081 & -114.993806 \\
\hline 3.1 & 4.0 & -114.996370 & -115.007141 & -115.005997 & -115.005341 & -115.006997 \\
\hline 3.1 & 5.0 & -114.985965 & -114.996525 & -114.9 & -114.994577 & -114.996453 \\
\hline 3.1 & 6.0 & -114.958011 & -114.969292 & -114.967203 & -114.966612 & -114.969249 \\
\hline 3.1 & 8.0 & -114.926807 & -114.942236 & -114.936037 & -114.935470 & -114.940524 \\
\hline 3.3 & 1.2 & -114.697986 & -114.706622 & -114.705519 & -114.705058 & -114.704726 \\
\hline 3.3 & 1.4 & -114.837504 & -114.847139 & -114.845885 & -114.845354 & -114.845088 \\
\hline 3.3 & 1.6 & -114.888594 & -114.899524 & -114.898077 & -114.897456 & -114.897758 \\
\hline 3.3 & 1.7325 & -114.899931 & -114.911910 & -114.910306 & -114.909615 & -114.910438 \\
\hline 3.3 & 1.8 & -114.902274 & -114.914841 & -114.913149 & -114.912417 & -114.913551 \\
\hline 3.3 & 2.0 & -114.903646 & -114.918023 & -114.916058 & -114.915200 & -114.917311 \\
\hline 3.3 & 2.25 & -114.904964 & -114.921073 & -114.918828 & -114.917852 & -114.920848 \\
\hline 3.3 & 2.5 & -114.911433 & -114.927942 & -114.925604 & -114.924623 & -114.927789 \\
\hline 3.3 & 2.75 & -114.922451 & -114.938117 & -114.935934 & -114.935044 & -114.937856 \\
\hline 3.3 & 3.0 & -114.935715 & -114.950039 & -114.948153 & -114.947364 & -114.949716 \\
\hline 3.3 & 3.5 & -114.959789 & -114.972062 & -114.970627 & -114.969934 & -114.971804 \\
\hline 3.3 & 4.0 & -114.972429 & -114.983764 & -114.982488 & -114.981825 & -114.983587 \\
\hline 3.3 & 5.0 & -114.964352 & -114.975343 & -114.973924 & -114.973288 & -114.975266 \\
\hline 3.3 & 6.0 & -114.938222 & -114.949912 & -114.947734 & -114.947133 & -114.949892 \\
\hline 3.3 & 8.0 & -114.907211 & -114.923042 & -114.916788 & -114.916224 & -114.921473 \\
\hline 3.5 & 1.2 & -114.712647 & -114.721057 & -114.719986 & -114.719536 & -114.718873 \\
\hline 3.5 & 1.4 & -114.852478 & -114.861854 & -114.860637 & -114.860119 & -114.859782 \\
\hline
\end{tabular}


Table S6. (continued)

\begin{tabular}{|c|c|c|c|c|c|c|}
\hline$R_{\mathrm{Be}-\mathrm{F}}$ & $R_{\mathrm{H}-\mathrm{F}}$ & CCSD & $\operatorname{CCSD}(\mathrm{T})$ & R-CCSD $(\mathrm{T})$ & CR-CCSD $(\mathrm{T})$ & $\operatorname{MRCI}(\mathrm{Q})$ \\
\hline 3.5 & 1.6 & -114.903045 & -114.913660 & -114.912256 & -114.911655 & -114.911826 \\
\hline 3.5 & 1.7325 & -114.913324 & -114.924943 & -114.923387 & -114.922720 & -114.923360 \\
\hline 3.5 & 1.8 & -114.914859 & -114.927046 & -114.925404 & -114.924700 & -114.925625 \\
\hline 3.5 & 2.0 & -114.912722 & -114.926742 & -114.924816 & -114.923991 & -114.925874 \\
\hline 3.5 & 2.25 & -114.907869 & -114.923993 & -114.921714 & -114.920753 & -114.923692 \\
\hline 3.5 & 2.5 & -114.907509 & -114.924693 & -114.922186 & -114.921177 & -114.924562 \\
\hline 3.5 & 2.75 & -114.912004 & -114.928889 & -114.926394 & -114.925454 & -114.928644 \\
\hline 3.5 & 3.0 & -114.919755 & -114.935419 & -114.933175 & -114.932353 & -114.935045 \\
\hline 3.5 & 3.5 & -114.937212 & -114.950417 & -114.948737 & -114.948046 & -114.950071 \\
\hline 3.5 & 4.0 & -114.947986 & -114.959953 & -114.958516 & -114.957858 & -114.959718 \\
\hline 3.5 & 5.0 & -114.941667 & -114.953108 & -114.951577 & -114.950939 & -114.953014 \\
\hline 3.5 & 6.0 & -114.917494 & -114.929609 & -114.927331 & -114.926725 & -114.929605 \\
\hline 3.5 & 8.0 & -114.886895 & -114.903187 & -114.896855 & -114.896295 & -114.901738 \\
\hline 3.7 & 1.2 & -114.724743 & -114.732881 & -114.731850 & -114.731414 & -114.731418 \\
\hline 3.7 & 1.4 & -114.864750 & -114.873810 & -114.872640 & -114.872139 & -114 . \\
\hline 3.7 & 1.6 & -114.914787 & -114.925019 & -114.923672 & -114.923093 & -114.922954 \\
\hline 3.7 & 1.7325 & -114.924151 & -114.935328 & -114.933838 & -114.933198 & -114.933665 \\
\hline 3.7 & 1.8 & -114.925009 & -114.936723 & -114.9 & -114.934477 & -114 \\
\hline 3.7 & 2.0 & -114.919966 & -114.933464 & 31613 & 930828 & -114 \\
\hline 3.7 & 2.25 & -114.909920 & -114.925727 & -114.923484 & -114.922559 & -114.925329 \\
\hline 3.7 & 2.5 & -114.903677 & -114.921125 & -114.918544 & -114.917535 & -114.921004 \\
\hline 3.7 & 2.75 & -114.902434 & -114.920279 & -114.917543 & -114.916563 & -114.920074 \\
\hline 3.7 & 3.0 & -114.905069 & -114.922098 & -114 & -114.918626 & -114.921696 \\
\hline 3.7 & 3.5 & -114.915699 & -114.930012 & -114.928021 & -114.927344 & -114.929534 \\
\hline 3.7 & 4.0 & -114.924042 & -114.936733 & -114.935095 & -114.934455 & -114.936400 \\
\hline 3.7 & 5.0 & -114.918887 & -114.930803 & -114.929141 & -114.9 & -114.930667 \\
\hline 3.7 & 6.0 & -114.896740 & -114.909299 & -114.906907 & -114.906302 & -114.909294 \\
\hline 3.7 & 8.0 & -114.866772 & -114.883575 & -114.877146 & -114.876590 & -114.882221 \\
\hline 3.9 & 1.2 & -114.734757 & -114.742596 & -114.741609 & -114.741188 & -114.740969 \\
\hline 3.9 & 1.4 & -114.874923 & -114.8 & -114.882517 & -114.882035 & -114.881566 \\
\hline 3.9 & 1.6 & -114.924527 & -114.934337 & -114.933053 & -114.932498 & -114.932438 \\
\hline 3.9 & 1.7325 & -114.933141 & -114.943831 & -114.942414 & -114.941803 & -114.942115 \\
\hline 3.9 & 1.8 & -114.933447 & -114.944636 & -114.943143 & -114.942502 & -114.943039 \\
\hline 3.9 & 2.0 & -114.926029 & -114.938905 & -114.937148 & -114.936406 & -114.937759 \\
\hline 3.9 & 2.25 & -114.911628 & -114.926868 & -114.924712 & -114.923835 & -114.926350 \\
\hline 3.9 & 2.5 & -114.900290 & -114.917592 & -114.915025 & -114.914043 & -114.917457 \\
\hline 3.9 & 2.75 & -114.894003 & -114.912402 & -114.909530 & -114.908532 & -114.912258 \\
\hline 3.9 & 3.0 & -114.891994 & -114.910225 & -114.907292 & -114.906395 & -114.909835 \\
\hline 3.9 & 3.5 & -114.895771 & -114.911392 & -114.909014 & -114.908361 & -114.910733 \\
\hline 3.9 & 4.0 & -114.901187 & -114.914729 & -114.912836 & -114.912232 & -114.914239 \\
\hline 3.9 & 5.0 & -114.896590 & -114.909018 & -114.907201 & -114.906587 & -114.908802 \\
\hline 3.9 & 6.0 & -114.876476 & -114.889504 & -114.886981 & -114.886383 & -114.889473 \\
\hline 3.9 & 8.0 & -114.847351 & -114.864717 & -114.858169 & -114.857620 & -114.863432 \\
\hline 4.1 & 1.2 & -114.742959 & -114.750498 & -114.749553 & -114.749148 & -114.748737 \\
\hline 4.1 & 1.4 & -114.883300 & -114.891658 & -114.890591 & -114.890128 & -114.890025 \\
\hline 4.1 & 1.6 & -114.932591 & -114.941970 & -114.940751 & -114.940220 & -114.940067 \\
\hline 4.1 & 1.7325 & -114.940626 & -114.950817 & -114.949474 & -114.948892 & -114.949069 \\
\hline 4.1 & 1.8 & -114.940498 & -114.951149 & -114.949737 & -114.949127 & -114.949502 \\
\hline 4.1 & 2.0 & -114.931186 & -114.943404 & -114.941747 & -114.941047 & -114.942149 \\
\hline
\end{tabular}


Table S6. (continued)

\begin{tabular}{|c|c|c|c|c|c|c|}
\hline$R_{\mathrm{Be}-\mathrm{F}}$ & $R_{\mathrm{H}-\mathrm{F}}$ & CCSD & $\operatorname{CCSD}(\mathrm{T})$ & R-CCSD $(\mathrm{T})$ & CR-CCSD(T) & MRCI(Q) \\
\hline 4.1 & 2.25 & -114.913199 & -114.927724 & -114.925682 & -114.924857 & -114.927074 \\
\hline 4.1 & 2.5 & -114.897478 & -114.914306 & -114.911819 & -114.910882 & -114.914124 \\
\hline 4.1 & 2.75 & -114.886757 & -114.905264 & -114.902361 & -114.901372 & -114.905180 \\
\hline 4.1 & 3.0 & -114.880600 & -114.899686 & -114.896533 & -114.895605 & -114.899355 \\
\hline 4.1 & 3.5 & -114.877720 & -114.894825 & -114.891996 & -114.891367 & -114.893947 \\
\hline 4.1 & 4.0 & -114.879778 & -114.894343 & -114.892123 & -114.891580 & -114.893610 \\
\hline 4.1 & 5.0 & -114.875125 & -114.888115 & -114.886113 & -114.885529 & -114.887769 \\
\hline 4.1 & 6.0 & -114.856991 & -114.870526 & -114.867848 & -114.867264 & -114.870432 \\
\hline 4.1 & 8.0 & -114.828906 & -114.846901 & -114.840206 & -114.839666 & -114.845652 \\
\hline 4.5 & 1.2 & -114.754854 & -114.761850 & -114.760979 & -114.760601 & -114.759898 \\
\hline 4.5 & 1.4 & -114.895555 & -114.903265 & -114.902288 & -114.901860 & -114.901406 \\
\hline 4.5 & 1.6 & -114.944518 & -114.953103 & -114.951995 & -114.951509 & -114.951394 \\
\hline 4.5 & 1.7325 & -114.951803 & -114.961073 & -114.959862 & -114.959333 & -114.959292 \\
\hline 4.5 & 1.8 & -114.951095 & -114.960751 & -114.959481 & -114.958929 & -114.959045 \\
\hline 4.5 & 2.0 & -114.939175 & -114.950144 & -114.948671 & -114.948045 & -114.948727 \\
\hline 4.5 & 2.25 & -114.915995 & -114.928988 & -114.927184 & -114.926457 & -114 . \\
\hline 4.5 & 2.5 & -114.893479 & -114.908821 & -114.906589 & -114.905756 & -114.908439 \\
\hline 4.5 & 2.75 & -114.875494 & -114.893161 & -114.890421 & -114.889503 & -114.893090 \\
\hline 4.5 & 3.0 & -114.862419 & -114.881909 & -114.878655 & -114 & -114 \\
\hline 4.5 & 3.5 & -114.847611 & -114.867729 & -114.863993 & -114.863357 & -114.866440 \\
\hline 4.5 & 4.0 & -114.842087 & -114.859475 & -114.856304 & -114.855966 & -114.857841 \\
\hline 4.5 & 5.0 & -114.835421 & -114.849799 & -114.847283 & -114.846827 & -114.848927 \\
\hline 4.5 & 6.0 & -114.820901 & -114.8 & -114.832557 & 32028 & -114 \\
\hline 4.5 & 8.0 & -114.795409 & -114.814986 & -114.807854 & -114.807332 & -114.813635 \\
\hline 4.7 & 1.2 & -114.758989 & -114.765757 & -114.764915 & -114.764550 & -114.762774 \\
\hline 4.7 & 1.4 & -114.899855 & -114.907291 & -114.906351 & -114.905938 & -114.904000 \\
\hline 4.7 & 1.6 & -114.948751 & -114.9 & -114.955936 & -114 . & -114 \\
\hline 4.7 & 1.7325 & -114.955810 & -114.964687 & -114.963530 & -114.963023 & -114.963009 \\
\hline 4.7 & 1.8 & -114.954918 & -114.964150 & -114.962938 & -114.962410 & -114.962430 \\
\hline 4.7 & 2.0 & -114.942139 & -114.952570 & -114.951172 & -114.950577 & -114.951098 \\
\hline 4.7 & 2.25 & -114.917161 & -114.929451 & -114.927751 & -114.927065 & -114.928416 \\
\hline 4.7 & 2.5 & -114.892126 & -114.906659 & -114.904559 & -114.903774 & -114.906141 \\
\hline 4.7 & 2.75 & -114.871250 & -114.888198 & -114.885593 & -114.884719 & -114.888060 \\
\hline 4.7 & 3.0 & -114.855313 & -114.874464 & -114.871285 & -114.870367 & -114.874335 \\
\hline 4.7 & 3.5 & -114.835434 & -114.856715 & -114.852644 & -114.851964 & -114.855329 \\
\hline 4.7 & 4.0 & -114.826007 & -114.845307 & -114.841487 & -114.841265 & -114.842904 \\
\hline 4.7 & 5.0 & -114.817353 & -114.832659 & -114.829775 & -114.829440 & -114.831313 \\
\hline 4.7 & 6.0 & -114.804387 & -114.819934 & -114.816540 & -114.816059 & -114.819179 \\
\hline 4.7 & 8.0 & -114.780403 & -114.801062 & -114.793583 & -114.793065 & -114.799497 \\
\hline 5.0 & 1.2 & -114.763493 & -114.769977 & -114.769171 & -114.768820 & -114.767043 \\
\hline 5.0 & 1.4 & -114.904567 & -114.911661 & -114.910765 & -114.910371 & -114.909526 \\
\hline 5.0 & 1.6 & -114.953427 & -114.961252 & -114.960246 & -114.959801 & -114.957721 \\
\hline 5.0 & 1.7325 & -114.960265 & -114.968650 & -114.967558 & -114.967079 & -114.966835 \\
\hline 5.0 & 1.8 & -114.959186 & -114.967884 & -114.966744 & -114.966246 & -114.966128 \\
\hline 5.0 & 2.0 & -114.945504 & -114.955254 & -114.953949 & -114.953392 & -114.953719 \\
\hline 5.0 & 2.25 & -114.918568 & -114.929945 & -114.928375 & -114.927739 & -114.928761 \\
\hline 5.0 & 2.5 & -114.890662 & -114.904068 & -114.902141 & -114.901419 & -114.903336 \\
\hline 5.0 & 2.75 & -114.866298 & -114.882077 & -114.879677 & -114.878866 & -114.881774 \\
\hline 5.0 & 3.0 & -114.846733 & -114.865009 & -114.862010 & -114.861128 & -114.864840 \\
\hline
\end{tabular}


Table S6. (continued)

\begin{tabular}{|c|c|c|c|c|c|c|}
\hline$R_{\mathrm{Be}-\mathrm{F}}$ & $R_{\mathrm{H}-\mathrm{F}}$ & CCSD & $\operatorname{CCSD}(\mathrm{T})$ & $\mathrm{R}-\mathrm{CCSD}(\mathrm{T})$ & CR-CCSD $(\mathrm{T})$ & $\operatorname{MRCI}(\mathrm{Q})$ \\
\hline 5.0 & 3.5 & -114.820324 & -114.842616 & -114.838264 & -114.837483 & -114.841222 \\
\hline 5.0 & 4.0 & -114.805426 & -114.828094 & -114.823144 & -114.822986 & -114.824110 \\
\hline 5.0 & 5.0 & -114.792541 & -114.809873 & -114.806171 & -114.806163 & -114.807258 \\
\hline 5.0 & 6.0 & -114.781517 & -114.798758 & -114.794692 & -114.794321 & -114.797047 \\
\hline 5.0 & 8.0 & -114.760006 & -114.783014 & -114.774697 & -114.774154 & -114.780698 \\
\hline 5.2 & 1.2 & -114.765615 & -114.771945 & -114.771159 & -114.770816 & -114.769040 \\
\hline 5.2 & 1.4 & -114.906801 & -114.913708 & -114.912836 & -114.912451 & -114.910507 \\
\hline 5.2 & 1.6 & -114.955658 & -114.963252 & -114.962275 & -114.961843 & -114.961105 \\
\hline 5.2 & 1.7325 & -114.962401 & -114.970518 & -114.969461 & -114.968996 & -114.968645 \\
\hline 5.2 & 1.8 & -114.961239 & -114.969646 & -114.968543 & -114.968061 & -114.967823 \\
\hline 5.2 & 2.0 & -114.947141 & -114.956518 & -114.955262 & -114.954726 & -114.954883 \\
\hline 5.2 & 2.25 & -114.919275 & -114.930144 & -114.928643 & -114.928035 & -114.928881 \\
\hline 5.2 & 2.5 & -114.889954 & -114.902704 & -114.900873 & -114.900185 & -114.901840 \\
\hline 5.2 & 2.75 & -114.863780 & -114.878810 & -114.876531 & -114.875756 & -114.878371 \\
\hline 5.2 & 3.0 & -114.842224 & -114.859812 & -114.856942 & -114.856085 & -114.859588 \\
\hline 5.2 & 3.5 & -114.812098 & -114.834620 & -114.830198 & -114.829344 & -114.833218 \\
\hline 5.2 & 4.0 & -114.794022 & -114.818978 & -114.813256 & -114.812995 & -114.813827 \\
\hline 5.2 & 5.0 & -114.777517 & -114.797061 & -114.792465 & -114.792849 & -114.792829 \\
\hline 5.2 & 6.0 & -114.767503 & -114.786540 & -114.781730 & -114.781477 & -114.783587 \\
\hline 5.2 & 8.0 & -114.747776 & -114.773250 & -114.763967 & -114.763362 & -114.769861 \\
\hline 5.5 & 1.2 & -114.767840 & -114.773982 & -114.773219 & -114.772886 & -114.771113 \\
\hline 5.5 & 1.4 & -114.909148 & -114.915829 & -114.914985 & -114.914612 & -114. \\
\hline 5.5 & 1.6 & -114.958011 & -114.965325 & -114.964384 & -114.963967 & -114.961880 \\
\hline 5.5 & 1.7325 & -114.964660 & -114.972450 & -114.971435 & -114.970988 & -114.970276 \\
\hline 5.5 & 1.8 & -114.963412 & -114.971465 & -114.970408 & -114.969945 & -114.969308 \\
\hline 5.5 & 2.0 & -114.948880 & -114.957802 & -114.956604 & -114.956093 & -114.956058 \\
\hline 5.5 & 2.25 & -114.920021 & -114.930267 & -114.928848 & -114.928273 & -114.928761 \\
\hline 5.5 & 2.5 & -114.889153 & -114.901074 & -114.899357 & -114.898710 & -114.900040 \\
\hline 5.5 & 2.75 & -114.860924 & -114.874947 & -114.872818 & -114.872090 & -114.874294 \\
\hline 5.5 & 3.0 & -114.836993 & -114.853546 & -114.850848 & -114.850027 & -114.853214 \\
\hline 5.5 & 3.5 & -114.802171 & -114.824591 & -114.820155 & -114.819198 & -114.823090 \\
\hline 5.5 & 4.0 & -114.780145 & -114.808098 & -114.801316 & -114.800667 & -114.804408 \\
\hline 5.5 & 5.0 & -114.757301 & -114.783460 & -114.776135 & -114.777519 & -114.773600 \\
\hline 5.5 & 6.0 & -114.748308 & -114.772618 & -114.765489 & -114.765507 & -114.765370 \\
\hline 5.5 & 8.0 & -114.731558 & -114.763776 & -114.751549 & -114.750631 & -114.759293 \\
\hline 6.0 & 1.2 & -114.769868 & -114.775790 & -114.775053 & -114.774732 & -114.772966 \\
\hline 6.0 & 1.4 & -114.911292 & -114.917706 & -114.916894 & -114.916535 & -114.914600 \\
\hline 6.0 & 1.6 & -114.960159 & -114.967141 & -114.966241 & -114.965842 & -114.963760 \\
\hline 6.0 & 1.7325 & -114.966718 & -114.974121 & -114.973154 & -114.972727 & -114.970572 \\
\hline 6.0 & 1.8 & -114.965387 & -114.973020 & -114.972015 & -114.971575 & -114.970821 \\
\hline 6.0 & 2.0 & -114.950435 & -114.958817 & -114.957688 & -114.957205 & -114.956646 \\
\hline 6.0 & 2.25 & -114.920613 & -114.930114 & -114.928789 & -114.928251 & -114.927976 \\
\hline 6.0 & 2.5 & -114.888218 & -114.899124 & -114.897537 & -114.896939 & -114.896962 \\
\hline 6.0 & 2.75 & -114.857894 & -114.870611 & -114.868656 & -114.867984 & -114.868283 \\
\hline 6.0 & 3.0 & -114.831414 & -114.846467 & -114.843978 & -114.843205 & -114.843663 \\
\hline 6.0 & 3.5 & -114.791082 & -114.812738 & -114.808333 & -114.807241 & -114.807755 \\
\hline 6.0 & 4.0 & -114.764422 & -114.795795 & -114.787537 & -114.785985 & -114.787323 \\
\hline 6.0 & 5.0 & -114.732469 & -114.796623 & -114.770385 & -114.769718 & -114.770474 \\
\hline 6.0 & 6.0 & -114.721759 & -114.790710 & -114.759199 & -114.758164 & -114.761372 \\
\hline
\end{tabular}


Table S6. (continued)

\begin{tabular}{lcccccc}
\hline$R_{\mathrm{Be}-\mathrm{F}}$ & $R_{\mathrm{H}-\mathrm{F}}$ & $\mathrm{CCSD}$ & $\mathrm{CCSD}(\mathrm{T})$ & $\mathrm{R}-\mathrm{CCSD}(\mathrm{T})$ & $\mathrm{CR}-\mathrm{CCSD}(\mathrm{T})$ & $\mathrm{MRCI}(\mathrm{Q})$ \\
\hline 6.0 & 8.0 & -114.712232 & -114.784660 & -114.749729 & -114.745286 & -114.755751 \\
8.0 & 1.2 & -114.770937 & -114.776541 & -114.775841 & -114.775537 & -114.773796 \\
8.0 & 1.4 & -114.912372 & -114.918399 & -114.917634 & -114.917296 & -114.915391 \\
8.0 & 1.6 & -114.961154 & -114.967658 & -114.966815 & -114.966442 & -114.964395 \\
8.0 & 1.7325 & -114.967574 & -114.974419 & -114.973520 & -114.973123 & -114.971006 \\
8.0 & 1.8 & -114.966140 & -114.973168 & -114.972237 & -114.971829 & -114.971027 \\
8.0 & 2.0 & -114.950732 & -114.958337 & -114.957302 & -114.956859 & -114.956206 \\
8.0 & 2.25 & -114.919964 & -114.928388 & -114.927193 & -114.926707 & -114.926285 \\
8.0 & 2.5 & -114.886159 & -114.895566 & -114.894159 & -114.893624 & -114.893470 \\
8.0 & 2.75 & -114.853981 & -114.864636 & -114.862928 & -114.862326 & -114.862484 \\
8.0 & 3.0 & -114.825323 & -114.837649 & -114.835484 & -114.834781 & -114.835341 \\
8.0 & 3.5 & -114.780625 & -114.798578 & -114.794504 & -114.793362 & -114.795228 \\
8.0 & 4.0 & -114.751419 & -114.780986 & -114.771701 & -114.769336 & -114.772650 \\
8.0 & 5.0 & -114.723767 & -114.818933 & -114.768255 & -114.756473 & -114.757335 \\
8.0 & 6.0 & -114.715672 & -114.963928 & -114.794971 & -114.761777 & -114.754762 \\
8.0 & 8.0 & -114.712864 & -115.157853 & -114.820172 & -114.769039 & -114.754064 \\
\hline
\end{tabular}

Table S7. The CCSD, CCSD(T), R-CCSD(T), CR-CCSD(T), and MRCI(Q) energies, in hartree, of the BeFH system, as described by the cc-pVTZ basis set, for the $\mathrm{Be}-\mathrm{F}-\mathrm{H}$ angle $\theta=0^{\circ}$ corresponding to the $\mathrm{Be}$ atom inserted between the $\mathrm{H}$ and $\mathrm{F}$ atoms. The $\mathrm{Be}-\mathrm{F}$ and Be-H bond distances, $R_{\mathrm{Be}-\mathrm{F}}$ and $R_{\mathrm{Be}-\mathrm{H}}$, respectively, are in bohr.

\begin{tabular}{lcccccc}
\hline$R_{\mathrm{Be}-\mathrm{F}}$ & $R_{\mathrm{Be}-\mathrm{H}}$ & $\mathrm{CCSD}$ & $\mathrm{CCSD}(\mathrm{T})$ & $\mathrm{R}-\mathrm{CCSD}(\mathrm{T})$ & $\mathrm{CR}-\mathrm{CCSD}(\mathrm{T})$ & $\mathrm{MRCI}(\mathrm{Q})$ \\
\hline 1.8 & 1.8 & -114.745898 & -114.753253 & -114.752776 & -114.752313 & -114.753201 \\
1.8 & 1.9 & -114.768271 & -114.775582 & -114.775107 & -114.774647 & -114.775533 \\
1.8 & 2.0 & -114.784883 & -114.792156 & -114.791683 & -114.791225 & -114.792110 \\
1.8 & 2.2 & -114.805184 & -114.812402 & -114.811927 & -114.811474 & -114.812358 \\
1.8 & 2.4 & -114.813433 & -114.820615 & -114.820136 & -114.819686 & -114.820567 \\
1.8 & 2.5 & -114.814422 & -114.821591 & -114.821107 & -114.820659 & -114.821537 \\
1.8 & 2.52 & -114.814422 & -114.821591 & -114.821107 & -114.820659 & -114.821537 \\
1.8 & 2.6 & -114.813852 & -114.821016 & -114.820528 & -114.820080 & -114.820958 \\
1.8 & 2.7 & -114.812031 & -114.819191 & -114.818698 & -114.818251 & -114.819129 \\
1.8 & 2.9 & -114.805618 & -114.812781 & -114.812272 & -114.811828 & -114.812711 \\
1.8 & 3.1 & -114.796743 & -114.803924 & -114.803395 & -114.802952 & -114.803850 \\
1.8 & 3.3 & -114.786476 & -114.793689 & -114.793133 & -114.792692 & -114.793615 \\
1.8 & 3.5 & -114.775541 & -114.782803 & -114.782212 & -114.781773 & -114.782732 \\
1.8 & 3.7 & -114.764427 & -114.771757 & -114.771122 & -114.770683 & -114.771693 \\
1.8 & 3.9 & -114.753472 & -114.760890 & -114.760199 & -114.759762 & -114.760837 \\
1.8 & 4.1 & -114.742914 & -114.750445 & -114.749682 & -114.749246 & -114.750404 \\
1.8 & 4.5 & -114.723617 & -114.731460 & -114.730495 & -114.730061 & -114.731441 \\
1.8 & 4.7 & -114.715077 & -114.723129 & -114.722023 & -114.721590 & -114.723112 \\
1.8 & 5.0 & -114.703789 & -114.712241 & -114.710859 & -114.710424 & -114.712197 \\
1.8 & 5.2 & -114.697293 & -114.706076 & -114.704457 & -114.704020 & -114.705983 \\
1.8 & 5.5 & -114.689042 & -114.698433 & -114.696367 & -114.695921 & -114.698194 \\
1.8 & 6.0 & -114.678885 & -114.689617 & -114.686520 & -114.686040 & -114.688848 \\
\hline
\end{tabular}


Table S7. (continued)

\begin{tabular}{|c|c|c|c|c|c|c|}
\hline$R_{\mathrm{Be}-\mathrm{F}}$ & $R_{\mathrm{Be}-\mathrm{H}}$ & CCSD & $\operatorname{CCSD}(\mathrm{T})$ & R-CCSD $(\mathrm{T})$ & CR-CCSD(T) & $\operatorname{MRCI}(\mathrm{Q})$ \\
\hline 1.8 & 8.0 & -114.664890 & -114.683124 & -114.673782 & -114.672946 & -114.677014 \\
\hline 1.9 & 1.8 & -114.844572 & -114.852064 & -114.851572 & -114.851095 & -114.852044 \\
\hline 1.9 & 1.9 & -114.867263 & -114.874714 & -114.874224 & -114.873750 & -114.874697 \\
\hline 1.9 & 2.0 & -114.884113 & -114.891530 & -114.891041 & -114.890569 & -114.891516 \\
\hline 1.9 & 2.2 & -114.904692 & -114.912059 & -114.911568 & -114.911100 & -114.912047 \\
\hline 1.9 & 2.4 & -114.913027 & -114.920362 & -114.919867 & -114.919402 & -114.920345 \\
\hline 1.9 & 2.5 & -114.914010 & -114.921334 & -114.920834 & -114.920370 & -114.921311 \\
\hline 1.9 & 2.52 & -114.914010 & -114.921334 & -114.920834 & -114.920370 & -114.921311 \\
\hline 1.9 & 2.6 & -114.913422 & -114.920741 & -114.920237 & -114.919774 & -114.920714 \\
\hline 1.9 & 2.7 & -114.911568 & -114.918885 & -114.918374 & -114.917913 & -114.918853 \\
\hline 1.9 & 2.9 & -114.905068 & -114.912389 & -114.911863 & -114.911404 & -114.912350 \\
\hline 1.9 & 3.1 & -114.896091 & -114.903432 & -114.902885 & -114.902427 & -114.903388 \\
\hline 1.9 & 3.3 & -114.885714 & -114.893088 & -114.892514 & -114.892058 & -114.893043 \\
\hline 1.9 & 3.5 & -114.874665 & -114.882089 & -114.881479 & -114.881025 & -114.882047 \\
\hline 1.9 & 3.7 & -114.863440 & -114.870930 & -114.870275 & -114.869823 & -114.870895 \\
\hline 1.9 & 3.9 & -114.852379 & -114.859957 & -114.859245 & -114.858794 & -114.859932 \\
\hline 1.9 & 4.1 & -114.841725 & -114.849413 & -114.848628 & -114.848179 & -114.849400 \\
\hline 1.9 & 4.5 & -114.822270 & -114.830265 & -114.829273 & -114.828827 & -114.830273 \\
\hline 1.9 & 4.7 & -114.813670 & -114.821869 & -114.820735 & -114.820290 & -114.821880 \\
\hline 1.9 & 5.0 & -114.802316 & -114.810903 & -114.809490 & -114.809046 & -114.810891 \\
\hline 1.9 & 5.2 & -114.795792 & -114.804700 & -114.803048 & -114.802603 & -114.804642 \\
\hline 1.9 & 5.5 & -114.787521 & -114.797018 & -114.794916 & -114.794465 & -114.796821 \\
\hline 1.9 & 6.0 & -114.777377 & -114.788166 & -114.785040 & -114.784560 & -114.787464 \\
\hline 1.9 & 8.0 & -114.763549 & -114.781422 & -114.772292 & -114.771489 & -114.775708 \\
\hline 2.0 & 1.8 & -114.914803 & -114.922436 & -114.921929 & -114.921438 & -114.922451 \\
\hline 2.0 & 1.9 & -114.937737 & -114.945332 & -114.944826 & -114.944338 & -114.945355 \\
\hline 2.0 & 2.0 & -114.954762 & -114.962327 & -114.961821 & -114.961335 & -114.962357 \\
\hline 2.0 & 2.2 & -114.975529 & -114.983048 & -114.982541 & -114.982059 & -114.983090 \\
\hline 2.0 & 2.4 & -114.983892 & -114.991384 & -114.990871 & -114.990392 & -114.991428 \\
\hline 2.0 & 2.5 & -114.984846 & -114.992328 & -114.991810 & -114.991332 & -114.992369 \\
\hline 2.0 & 2.52 & -114.984846 & -114.992328 & -114.991810 & -114.991332 & -114.992369 \\
\hline 2.0 & 2.6 & -114.984227 & -114.991705 & -114.991183 & -114.990706 & -114.991744 \\
\hline 2.0 & 2.7 & -114.982328 & -114.989805 & -114.989277 & -114.988800 & -114.989840 \\
\hline 2.0 & 2.9 & -114.975727 & -114.983211 & -114.982667 & -114.982193 & -114.983238 \\
\hline 2.0 & 3.1 & -114.966647 & -114.974152 & -114.973586 & -114.973113 & -114.974174 \\
\hline 2.0 & 3.3 & -114.956165 & -114.963705 & -114.963111 & -114.962640 & -114.963727 \\
\hline 2.0 & 3.5 & -114.945012 & -114.952602 & -114.951973 & -114.951503 & -114.952628 \\
\hline 2.0 & 3.7 & -114.933686 & -114.941344 & -114.940668 & -114.940200 & -114.941377 \\
\hline 2.0 & 3.9 & -114.922532 & -114.930278 & -114.929543 & -114.929077 & -114.930321 \\
\hline 2.0 & 4.1 & -114.911795 & -114.919650 & -114.918841 & -114.918377 & -114.919706 \\
\hline 2.0 & 4.5 & -114.892208 & -114.900365 & -114.899345 & -114.898886 & -114.900446 \\
\hline 2.0 & 4.7 & -114.883559 & -114.891917 & -114.890751 & -114.890294 & -114.892002 \\
\hline 2.0 & 5.0 & -114.872153 & -114.880891 & -114.879442 & -114.878987 & -114.880957 \\
\hline 2.0 & 5.2 & -114.865609 & -114.874659 & -114.872969 & -114.872515 & -114.874684 \\
\hline 2.0 & 5.5 & -114.857327 & -114.866950 & -114.864808 & -114.864350 & -114.866844 \\
\hline 2.0 & 6.0 & -114.847210 & -114.858083 & -114.854919 & -114.854438 & -114.857495 \\
\hline 2.0 & 8.0 & -114.833560 & -114.851145 & -114.842189 & -114.841417 & -114.845840 \\
\hline 2.2 & 1.8 & -114.996993 & -115.004913 & -115.004372 & -115.003853 & -115.004961 \\
\hline 2.2 & 1.9 & -115.020251 & -115.028139 & -115.027599 & -115.027082 & -115.028193 \\
\hline
\end{tabular}


Table S7. (continued)

\begin{tabular}{|c|c|c|c|c|c|c|}
\hline$R_{\mathrm{Be}-\mathrm{F}}$ & $R_{\mathrm{Be}-\mathrm{H}}$ & CCSD & $\operatorname{CCSD}(\mathrm{T})$ & R-CCSD $(\mathrm{T})$ & CR-CCSD $(\mathrm{T})$ & MRCI(Q) \\
\hline 2.2 & 2.0 & -115.037499 & -115.045362 & -115.044823 & -115.044307 & -115.045422 \\
\hline 2.2 & 2.2 & -115.058467 & -115.066295 & -115.065753 & -115.065240 & -115.066365 \\
\hline 2.2 & 2.4 & -115.066787 & -115.074595 & -115.074047 & -115.073537 & -115.074667 \\
\hline 2.2 & 2.5 & -115.067640 & -115.075443 & -115.074889 & -115.074379 & -115.075511 \\
\hline 2.2 & 2.52 & -115.067640 & -115.075443 & -115.074889 & -115.074379 & -115.075511 \\
\hline 2.2 & 2.6 & -115.066938 & -115.074740 & -115.074181 & -115.073672 & -115.074805 \\
\hline 2.2 & 2.7 & -115.064928 & -115.072732 & -115.072167 & -115.071659 & -115.072793 \\
\hline 2.2 & 2.9 & -115.058110 & -115.065926 & -115.065344 & -115.064837 & -115.065980 \\
\hline 2.2 & 3.1 & -115.048829 & -115.056671 & -115.056067 & -115.055562 & -115.056719 \\
\hline 2.2 & 3.3 & -115.038158 & -115.046041 & -115.045406 & -115.044903 & -115.046087 \\
\hline 2.2 & 3.5 & -115.026823 & -115.034761 & -115.034088 & -115.033586 & -115.034809 \\
\hline 2.2 & 3.7 & -115.015326 & -115.023335 & -115.022613 & -115.022113 & -115.023389 \\
\hline 2.2 & 3.9 & -115.004017 & -115.012115 & -115.011331 & -115.010833 & -115.012179 \\
\hline 2.2 & 4.1 & -114.993144 & -115.001352 & -115.000490 & -114.999995 & -115.001428 \\
\hline 2.2 & 4.5 & -114.973351 & -114.981858 & -114.980774 & -114.980286 & -114.981959 \\
\hline 2.2 & 4.7 & -114.964630 & -114.973334 & -114.972098 & -114.971614 & -114.973440 \\
\hline 2.2 & 5.0 & -114.953157 & -114.962229 & -114.960699 & -114.960221 & -114.962321 \\
\hline 2.2 & 5.2 & -114.946592 & -114.955967 & -114.954188 & -114.953713 & -114.956021 \\
\hline 2.2 & 5.5 & -114.938318 & -114.948242 & -114.946001 & -114.945527 & -114.948175 \\
\hline 2.2 & 6.0 & -114.928288 & -114.939397 & -114.936132 & -114.935645 & -114.938884 \\
\hline 2.2 & 8.0 & -114.915024 & -114.932227 & -114.923521 & -114.922803 & -114.927497 \\
\hline 2.4 & 1.8 & -115.031068 & -115.039277 & -115.038702 & -115.038155 & -115.039364 \\
\hline 2.4 & 1.9 & -115.054515 & -115.062699 & -115.062124 & -115.061579 & -115.062790 \\
\hline 2.4 & 2.0 & -115.071884 & -115.080048 & -115.079473 & -115.078929 & -115.080144 \\
\hline 2.4 & 2.2 & -115.092919 & -115.101058 & -115.100480 & -115.099938 & -115.101163 \\
\hline 2.4 & 2.4 & -115.101119 & -115.109248 & -115.108663 & -115.108121 & -115.109352 \\
\hline 2.4 & 2.5 & -115.101840 & -115.109968 & -115.109376 & -115.108836 & -115.110068 \\
\hline 2.4 & 2.52 & -115.101840 & -115.109968 & -115.109376 & -115.108836 & -115.110068 \\
\hline 2.4 & 2.6 & -115.101038 & -115.109168 & -115.108572 & -115.108032 & -115.109265 \\
\hline 2.4 & 2.7 & -115.098903 & -115.107038 & -115.106434 & -115.105895 & -115.107130 \\
\hline 2.4 & 2.9 & -115.091856 & -115.100011 & -115.099388 & -115.098850 & -115.100094 \\
\hline 2.4 & 3.1 & -115.082385 & -115.090572 & -115.089926 & -115.089389 & -115.090649 \\
\hline 2.4 & 3.3 & -115.071545 & -115.079779 & -115.079101 & -115.078565 & -115.079853 \\
\hline 2.4 & 3.5 & -115.060053 & -115.068347 & -115.067628 & -115.067094 & -115.068421 \\
\hline 2.4 & 3.7 & -115.048410 & -115.056780 & -115.056009 & -115.055476 & -115.056857 \\
\hline 2.4 & 3.9 & -115.036971 & -115.045435 & -115.044598 & -115.044068 & -115.045521 \\
\hline 2.4 & 4.1 & -115.025989 & -115.034566 & -115.033646 & -115.033119 & -115.034663 \\
\hline 2.4 & 4.5 & -115.006042 & -115.014920 & -115.013766 & -115.013249 & -115.015043 \\
\hline 2.4 & 4.7 & -114.997276 & -115.006351 & -115.005037 & -115.004525 & -115.006480 \\
\hline 2.4 & 5.0 & -114.985772 & -114.995214 & -114.993591 & -114.993087 & -114.995330 \\
\hline 2.4 & 5.2 & -114.979211 & -114.988952 & -114.987070 & -114.986571 & -114.989033 \\
\hline 2.4 & 5.5 & -114.970975 & -114.981253 & -114.978895 & -114.978402 & -114.981221 \\
\hline 2.4 & 6.0 & -114.961070 & -114.972492 & -114.969098 & -114.968602 & -114.972040 \\
\hline 2.4 & 8.0 & -114.948211 & -114.965269 & -114.956693 & -114.956022 & -114.960991 \\
\hline 2.5 & 1.8 & -115.037249 & -115.045604 & -115.045011 & -115.044450 & -115.045710 \\
\hline 2.5 & 1.9 & -115.060760 & -115.069092 & -115.068499 & -115.067940 & -115.069202 \\
\hline 2.5 & 2.0 & -115.078163 & -115.086478 & -115.085886 & -115.085327 & -115.086593 \\
\hline 2.5 & 2.2 & -115.099197 & -115.107493 & -115.106896 & -115.106339 & -115.107614 \\
\hline 2.5 & 2.4 & -115.107314 & -115.115603 & -115.114999 & -115.114443 & -115.115723 \\
\hline
\end{tabular}


Table S7. (continued)

\begin{tabular}{|c|c|c|c|c|c|c|}
\hline$R_{\mathrm{Be}-\mathrm{F}}$ & $R_{\mathrm{Be}-\mathrm{H}}$ & CCSD & $\operatorname{CCSD}(\mathrm{T})$ & R-CCSD $(\mathrm{T})$ & CR-CCSD(T) & MRCI(Q) \\
\hline 2.5 & 2.5 & -115.107958 & -115.116249 & -115.115638 & -115.115082 & -115.116364 \\
\hline 2.5 & 2.52 & -115.107958 & -115.116249 & -115.115638 & -115.115082 & -115.116364 \\
\hline 2.5 & 2.6 & -115.107100 & -115.115395 & -115.114779 & -115.114224 & -115.115506 \\
\hline 2.5 & 2.7 & -115.104897 & -115.113198 & -115.112574 & -115.112020 & -115.113304 \\
\hline 2.5 & 2.9 & -115.097732 & -115.106056 & -115.105413 & -115.104860 & -115.106153 \\
\hline 2.5 & 3.1 & -115.088169 & -115.096529 & -115.095861 & -115.095308 & -115.096619 \\
\hline 2.5 & 3.3 & -115.077252 & -115.085662 & -115.084961 & -115.084409 & -115.085747 \\
\hline 2.5 & 3.5 & -115.065689 & -115.074162 & -115.073419 & -115.072869 & -115.074247 \\
\hline 2.5 & 3.7 & -115.053981 & -115.062532 & -115.061735 & -115.061187 & -115.062621 \\
\hline 2.5 & 3.9 & -115.042485 & -115.051131 & -115.050267 & -115.049721 & -115.051228 \\
\hline 2.5 & 4.1 & -115.031454 & -115.040216 & -115.039266 & -115.038724 & -115.040324 \\
\hline 2.5 & 4.5 & -115.011441 & -115.020509 & -115.019318 & -115.018786 & -115.020643 \\
\hline 2.5 & 4.7 & -115.002659 & -115.011925 & -115.010569 & -115.010044 & -115.012066 \\
\hline 2.5 & 5.0 & -114.991150 & -115.000785 & -114.999112 & -114.998596 & -115.000914 \\
\hline 2.5 & 5.2 & -114.984599 & -114.994532 & -114. & 2085 & -114.994627 \\
\hline 2.5 & 5.5 & -114.976392 & -114.986861 & -114.984438 & -114.983936 & -114.986845 \\
\hline 2.5 & 6.0 & -114.966560 & -114.978161 & -114.974694 & -114.974193 & -114.977735 \\
\hline 2.5 & 8.0 & 3904 & 70967 & -114 & 1766 & -114 \\
\hline 2.5719 & 1.8 & 38649 & 47109 & -115 & 6933 & -115 \\
\hline 2.5719 & 1.9 & -115.062195 & -115.070634 & -115.070028 & -115.069459 & -115.070757 \\
\hline 2.5719 & 2.0 & -115.079616 & -115.088039 & -115.087433 & -115.086865 & -115.088166 \\
\hline 2.5719 & 2.2 & 637 & -115 . & -115 & -115.107867 & -115.109177 \\
\hline 2.5719 & 2.4 & -115.108685 & -115 . & -115 & -115 & 17220 \\
\hline 2.5719 & 2.5 & -115.109269 & -115.117677 & -115.117052 & -115.116487 & -115.117803 \\
\hline 2.5719 & 2.52 & -115.109269 & -115.117677 & -115.117052 & -115.116487 & -115.117803 \\
\hline 5719 & 2.6 & -115 & -115.116782 & -115 & -115.115587 & -115.116904 \\
\hline 2.5719 & 2.7 & -115.106116 & -115.114536 & 13898 & -115.113333 & -115.114652 \\
\hline 2.5719 & 2.9 & -115.098866 & -115.107311 & -115.106653 & -115.106089 & -115.107418 \\
\hline 2.5719 & 3.1 & -115.089240 & -115.097723 & -115.097040 & -115.096476 & -115.097822 \\
\hline 2.5719 & 3.3 & -115. & -115. & -115 & -115 & -115 . \\
\hline 2.5719 & 3.5 & -115.066662 & -115.075262 & -115.074501 & -115.073940 & -115.075355 \\
\hline 2.5719 & 3.7 & -115.054910 & -115.063590 & -115.062774 & -115.062216 & -115.063688 \\
\hline 2.5719 & 3.9 & -115.043374 & -115.052153 & -115.051267 & -115.050712 & -115.052258 \\
\hline 2.5719 & 4.1 & -115.032311 & -115.041206 & -115 & -115.039682 & -115.041323 \\
\hline 2.5719 & 4.5 & -115.012254 & -115.021459 & -115.020240 & -115.019699 & -115.021600 \\
\hline 2.5719 & 4.7 & -115.003462 & -115.012867 & -115.011480 & -115.010945 & -115.013015 \\
\hline 2.5719 & 5.0 & -114.991953 & -115.001728 & -115.000018 & -114.999494 & -115.001866 \\
\hline 2.5719 & 5.2 & -114.985411 & -114.995485 & -114.993506 & -114.992989 & -114.995590 \\
\hline 2.5719 & 5.5 & -114.977227 & -114.987838 & -114.985368 & -114.984859 & -114.987834 \\
\hline 2.5719 & 6.0 & -114.967452 & -114.979190 & -114.975668 & -114.975163 & -114.978782 \\
\hline 2.5719 & 8.0 & -114.954942 & -114.972038 & -114.963492 & -114.962853 & -114.968057 \\
\hline 2.6 & 1.8 & -115.038633 & -115.047133 & -115.046522 & -115.045949 & -115.047258 \\
\hline 2.6 & 1.9 & -115.062190 & -115.070670 & -115.070060 & -115.069487 & -115.070799 \\
\hline 2.6 & 2.0 & -115.079616 & -115.088082 & -115.087471 & -115.086899 & -115.088213 \\
\hline 2.6 & 2.2 & -115.100630 & -115.109081 & -115.108465 & -115.107894 & -115.109218 \\
\hline 2.6 & 2.4 & -115.108650 & -115.117099 & -115.116475 & -115.115905 & -115.117235 \\
\hline 2.6 & 2.5 & -115.109210 & -115.117664 & -115.117033 & -115.116463 & -115.117793 \\
\hline 2.6 & 2.52 & -115.109210 & -115.117664 & -115.117033 & -115.116463 & -115.117793 \\
\hline 2.6 & 2.6 & -115.108295 & -115.116752 & -115.116116 & -115.115547 & -115.116878 \\
\hline
\end{tabular}


Table S7. (continued)

\begin{tabular}{|c|c|c|c|c|c|c|}
\hline$R_{\mathrm{Be}-\mathrm{F}}$ & $R_{\mathrm{Be}-\mathrm{H}}$ & CCSD & $\operatorname{CCSD}(\mathrm{T})$ & $\mathrm{R}-\mathrm{CCSD}(\mathrm{T})$ & CR-CCSD(T) & MRCI(Q) \\
\hline 2.6 & 2.7 & -115.106020 & -115.114486 & -115.113842 & -115.113274 & -115.114607 \\
\hline 2.6 & 2.9 & -115.098738 & -115.107230 & -115.106566 & -115.105998 & -115.107340 \\
\hline 2.6 & 3.1 & -115.089088 & -115.097619 & -115.096929 & -115.096362 & -115.097721 \\
\hline 2.6 & 3.3 & -115.078101 & -115.086684 & -115.085960 & -115.085394 & -115.086781 \\
\hline 2.6 & 3.5 & -115.066473 & -115.075122 & -115.074355 & -115.073790 & -115.075219 \\
\hline 2.6 & 3.7 & -115.054704 & -115.063435 & -115.062612 & -115.062050 & -115.063536 \\
\hline 2.6 & 3.9 & -115.043154 & -115.051984 & -115.051090 & -115.050531 & -115.052092 \\
\hline 2.6 & 4.1 & -115.032078 & -115.041026 & -115.040044 & -115.039488 & -115.041146 \\
\hline 2.6 & 4.5 & -115.012005 & -115.021264 & -115.020034 & -115.019488 & -115.021409 \\
\hline 2.6 & 4.7 & -115.003209 & -115.012668 & -115.011269 & -115.010730 & -115.012820 \\
\hline 2.6 & 5.0 & -114.991701 & -115.001531 & -114.999807 & -114.999279 & -115.001673 \\
\hline 2.6 & 5.2 & -114.985162 & -114.995293 & -114.993297 & -114.992777 & -114.995402 \\
\hline 2.6 & 5.5 & -114.976989 & -114.987657 & -114.985167 & -114.984656 & -114.987657 \\
\hline 2.6 & 6.0 & -114.967237 & -114.979030 & -114.975486 & -114.974979 & -114.978628 \\
\hline 2.6 & 8.0 & -114.954783 & -114.971898 & -114.963351 & -114.962717 & -114.967958 \\
\hline 2.7 & 1.8 & -115.036472 & -115.045118 & -115.044489 & -115.043903 & -115.045261 \\
\hline 2.7 & 1.9 & -115.060063 & -115.068691 & -115.068062 & -115.067477 & -115.068837 \\
\hline 2.7 & 2.0 & -115.077501 & -115.086117 & -115.085487 & -115.084902 & -115.086265 \\
\hline 2.7 & 2.2 & -115.098480 & -115.107086 & -115.106451 & -115.105868 & -115.107240 \\
\hline 2.7 & 2.4 & -115.106396 & -115.115004 & -115.114360 & -115.113777 & -115.115154 \\
\hline 2.7 & 2.5 & -115.106871 & -115.115484 & -115.114833 & -115.114251 & -115.115628 \\
\hline 2.7 & 2.52 & -115.106871 & -115.115484 & -115.114833 & -115.114251 & -115.115628 \\
\hline 2.7 & 2.6 & -115.105895 & -115.114515 & -115.113858 & -115.113276 & -115.114654 \\
\hline 2.7 & 2.7 & -115.103550 & -115.112179 & -115.111515 & -115.110932 & -115.112313 \\
\hline 2.7 & 2.9 & -115.096156 & -115.104813 & -115.104128 & -115.103547 & -115.104937 \\
\hline 2.7 & 3.1 & -115.086429 & -115.095128 & -115.094416 & -115.093835 & -115.095243 \\
\hline 2.7 & 3.3 & -115.075379 & -115.084134 & -115.083387 & -115.082807 & -115.084243 \\
\hline 2.7 & 3.5 & -115.063693 & -115.072518 & -115.071725 & -115.071147 & -115.072626 \\
\hline 2.7 & 3.7 & -115.051868 & -115.060778 & -115.059928 & -115.059352 & -115.060890 \\
\hline 2.7 & 3.9 & -115.040267 & -115.049278 & -115.048356 & -115.047783 & -115.049398 \\
\hline 2.7 & 4.1 & -115.029147 & -115.038280 & -115.037266 & -115.036697 & -115.038411 \\
\hline 2.7 & 4.5 & -115.009017 & -115.018467 & -115.017197 & -115.016639 & -115.018624 \\
\hline 2.7 & 4.7 & -115.000210 & -115.009864 & -115.008420 & -115.007869 & -115.010029 \\
\hline 2.7 & 5.0 & -114.988707 & -114.998736 & -114.996958 & -114.996420 & -114.998892 \\
\hline 2.7 & 5.2 & -114.982185 & -114.992517 & -114.990461 & -114.989931 & -114.992641 \\
\hline 2.7 & 5.5 & -114.974051 & -114.984923 & -114.982363 & -114.981844 & -114.984940 \\
\hline 2.7 & 6.0 & -114.964382 & -114.976380 & -114.972753 & -114.972241 & -114.976000 \\
\hline 2.7 & 8.0 & -114.952126 & -114.969337 & -114.960771 & -114.960153 & -114.965528 \\
\hline 2.9 & 1.8 & -115.025027 & -115.033961 & -115.033295 & -115.032686 & -115.034139 \\
\hline 2.9 & 1.9 & -115.048657 & -115.057578 & -115.056911 & -115.056304 & -115.057758 \\
\hline 2.9 & 2.0 & -115.066098 & -115.075012 & -115.074344 & -115.073736 & -115.075193 \\
\hline 2.9 & 2.2 & -115.086992 & -115.095903 & -115.095229 & -115.094622 & -115.096088 \\
\hline 2.9 & 2.4 & -115.094702 & -115.103621 & -115.102937 & -115.102331 & -115.103800 \\
\hline 2.9 & 2.5 & -115.095013 & -115.103941 & -115.103249 & -115.102643 & -115.104114 \\
\hline 2.9 & 2.52 & -115.095013 & -115.103941 & -115.103249 & -115.102643 & -115.104114 \\
\hline 2.9 & 2.6 & -115.093927 & -115.102863 & -115.102165 & -115.101559 & -115.103030 \\
\hline 2.9 & 2.7 & -115.091458 & -115.100406 & -115.099699 & -115.099093 & -115.100567 \\
\hline 2.9 & 2.9 & -115.083883 & -115.092864 & -115.092136 & -115.091530 & -115.093015 \\
\hline 2.9 & 3.1 & -115.074039 & -115.083069 & -115.082311 & -115.081706 & -115.083209 \\
\hline
\end{tabular}


Table S7. (continued)

\begin{tabular}{|c|c|c|c|c|c|c|}
\hline$R_{\mathrm{Be}-\mathrm{F}}$ & $R_{\mathrm{Be}-\mathrm{H}}$ & CCSD & $\operatorname{CCSD}(\mathrm{T})$ & R-CCSD(T) & CR-CCSD $(\mathrm{T})$ & $\operatorname{MRCI}(\mathrm{Q})$ \\
\hline 2.9 & 3.3 & -115.062886 & -115.071980 & -115.071185 & -115.070580 & -115.072115 \\
\hline 2.9 & 3.5 & -115.051096 & -115.060267 & -115.059423 & -115.058821 & -115.060400 \\
\hline 2.9 & 3.7 & -115.039168 & -115.048431 & -115.047526 & -115.046926 & -115.048568 \\
\hline 2.9 & 3.9 & -115.027470 & -115.036843 & -115.035860 & -115.035263 & -115.036987 \\
\hline 2.9 & 4.1 & -115.016268 & -115.025769 & -115.024690 & -115.024097 & -115.025925 \\
\hline 2.9 & 4.5 & -114.996036 & -115.005870 & -115.004518 & -115.003938 & -115.006054 \\
\hline 2.9 & 4.7 & -114.987214 & -114.997260 & -114.995724 & -114.995151 & -114.997454 \\
\hline 2.9 & 5.0 & -114.975734 & -114.986169 & -114.984280 & -114.983722 & -114.986356 \\
\hline 2.9 & 5.2 & -114.969253 & -114.980001 & -114.977820 & -114.977272 & -114.980157 \\
\hline 2.9 & 5.5 & -114.961209 & -114.972509 & -114.969804 & -114.969269 & -114.972560 \\
\hline 2.9 & 6.0 & -114.951720 & -114.964158 & -114.960356 & -114.959835 & -114.963818 \\
\hline 2.9 & 8.0 & -114.939838 & -114.957362 & -114.948701 & -114.948108 & -114.953750 \\
\hline 3.1 & 1.8 & -115.007975 & -115.017193 & -115.016489 & -115.015862 & -115.017405 \\
\hline 3.1 & 1.9 & -115.031623 & -115.040831 & -115.040126 & -115.039499 & -115.041043 \\
\hline 3.1 & 2.0 & -115.049056 & -115.058259 & -115.057552 & -115.056926 & 58472 \\
\hline 3.1 & 2.2 & -115.069861 & -115.079068 & -115.078354 & -115.077728 & -115.079285 \\
\hline 3.1 & 2.4 & -115.077394 & -115.086616 & -115.085891 & -115.085265 & -115.086825 \\
\hline 3.1 & 2.5 & -115.077575 & -115.086810 & -115.086076 & -115. & -115 . \\
\hline 3.1 & 2.52 & -115.077575 & -115.086810 & -115.086076 & 85450 & -115 \\
\hline 3.1 & 2.6 & -115.076409 & -115.085653 & -115.084913 & -115.084287 & -115.085850 \\
\hline 3.1 & 2.7 & -115.073857 & -115.083116 & -115.082367 & -115.081741 & -115.083306 \\
\hline 3.1 & 2.9 & -115.066175 & -115.075476 & 74703 & -115.074077 & -115.075654 \\
\hline 3.1 & 3.1 & -115.056251 & -115.065610 & -115.064805 & -115.064180 & 65778 \\
\hline 3.1 & 3.3 & -115.045010 & -115.054441 & -115.053595 & -115.052971 & -115.054603 \\
\hline 3.1 & 3.5 & -115.033123 & -115.042640 & -115.041742 & -115.041119 & -115.042800 \\
\hline 3.1 & 3.7 & -115.021099 & -115.030716 & -115.029754 & -115.029133 & -115.030880 \\
\hline 3.1 & 3.9 & -115.009316 & -115.019050 & -115.018005 & -115.017388 & -115.019223 \\
\hline 3.1 & 4.1 & -114.998044 & -115.007916 & -115.006768 & -115.006155 & -115.008101 \\
\hline 3.1 & 4.5 & -114.977739 & -114.987963 & -114.986525 & -114.985927 & -114.988180 \\
\hline 3.1 & 4.7 & -114.968917 & -114.979365 & -114.977731 & -114.977141 & -114.979593 \\
\hline 3.1 & 5.0 & -114.957481 & -114.968336 & -114.966330 & -114.965756 & -114.968559 \\
\hline 3.1 & 5.2 & -114.951053 & -114.962234 & -114.959922 & -114.959359 & -114.962427 \\
\hline 3.1 & 5.5 & -114.943111 & -114.954865 & -114.952005 & -114.951457 & -114.954954 \\
\hline 3.1 & 6.0 & -114.933805 & -114.946727 & -114.942738 & -114.942208 & -114.946425 \\
\hline 3.1 & 8.0 & -114.922262 & -114.940249 & -114.931421 & -114.930847 & -114.936756 \\
\hline 3.3 & 1.8 & -114.988274 & -114.997769 & -114.997026 & -114.996384 & -114.998014 \\
\hline 3.3 & 1.9 & -115.011926 & -115.021414 & -115.020670 & -115.020028 & -115.021658 \\
\hline 3.3 & 2.0 & -115.029343 & -115.038829 & -115.038082 & -115.037441 & -115.039073 \\
\hline 3.3 & 2.2 & -115.050062 & -115.059557 & -115.058802 & -115.058161 & -115.059804 \\
\hline 3.3 & 2.4 & -115.057449 & -115.066965 & -115.066198 & -115.065557 & -115.067203 \\
\hline 3.3 & 2.5 & -115.057541 & -115.067075 & -115.066298 & -115.065657 & -115.067305 \\
\hline 3.3 & 2.52 & -115.057541 & -115.067075 & -115.066298 & -115.065657 & -115.067305 \\
\hline 3.3 & 2.6 & -115.056328 & -115.065874 & -115.065091 & -115.064450 & -115.066101 \\
\hline 3.3 & 2.7 & -115.053733 & -115.063299 & -115.062505 & -115.061864 & -115.063519 \\
\hline 3.3 & 2.9 & -115.045992 & -115.055610 & -115.054790 & -115.054148 & -115.055816 \\
\hline 3.3 & 3.1 & -115.035997 & -115.045681 & -115.044827 & -115.044186 & -115.045878 \\
\hline 3.3 & 3.3 & -115.024668 & -115.034434 & -115.033536 & -115.032895 & -115.034624 \\
\hline 3.3 & 3.5 & -115.012690 & -115.022550 & -115.021597 & -115.020959 & -115.022740 \\
\hline 3.3 & 3.7 & -115.000584 & -115.010553 & -115.009531 & -115.008894 & -115.010747 \\
\hline
\end{tabular}


Table S7. (continued)

\begin{tabular}{|c|c|c|c|c|c|c|}
\hline$R_{\mathrm{Be}-\mathrm{F}}$ & $R_{\mathrm{Be}-\mathrm{H}}$ & CCSD & $\operatorname{CCSD}(\mathrm{T})$ & $\mathrm{R}-\mathrm{CCSD}(\mathrm{T})$ & CR-CCSD $(\mathrm{T})$ & $\operatorname{MRCI}(\mathrm{Q})$ \\
\hline 3.3 & 3.9 & -114.988733 & -114.998829 & -114.997718 & -114.997086 & -114.999032 \\
\hline 3.3 & 4.1 & -114.977413 & -114.987656 & -114.986436 & -114.985809 & -114.987873 \\
\hline 3.3 & 4.5 & -114.957077 & -114.967696 & -114.966168 & -114.965556 & -114.967947 \\
\hline 3.3 & 4.7 & -114.948274 & -114.959130 & -114.957394 & -114.956792 & -114.959395 \\
\hline 3.3 & 5.0 & -114.936902 & -114.948190 & -114.946061 & -114.945476 & -114.948452 \\
\hline 3.3 & 5.2 & -114.930537 & -114.942167 & -114.939718 & -114.939144 & -114.942401 \\
\hline 3.3 & 5.5 & -114.922703 & -114.934935 & -114.931913 & -114.931357 & -114.935063 \\
\hline 3.3 & 6.0 & -114.913577 & -114.927022 & -114.922833 & -114.922297 & -114.926754 \\
\hline 3.3 & 8.0 & -114.902331 & -114.920908 & -114.911854 & -114.911293 & -114.917467 \\
\hline 3.5 & 1.8 & -114.967657 & -114.977423 & -114.976639 & -114.975987 & -114.977697 \\
\hline 3.5 & 1.9 & -114.991303 & -115.001064 & -115.000279 & -114.999627 & -115.001337 \\
\hline 3.5 & 2.0 & -115.008697 & -115.018459 & -115.017671 & -115.017019 & -115.018732 \\
\hline 3.5 & 2.2 & -115.029326 & -115.039102 & -115.038305 & -115.037653 & -115.039377 \\
\hline 3.5 & 2.4 & -115.036593 & -115.046398 & -115.045587 & -115.044935 & -115.046663 \\
\hline 3.5 & 2.5 & -115.036629 & -115.046457 & -115.045636 & -115.044983 & -115.046715 \\
\hline 3.5 & 2.52 & -115.036629 & -115.046457 & -115.045636 & -115.044983 & -115.046715 \\
\hline 3.5 & 2.6 & -115.035391 & -115.045235 & -115.044407 & -115.043754 & -115.045489 \\
\hline 3.5 & 2.7 & -115.032774 & -115.042643 & -115.041803 & -115.041151 & -115.042890 \\
\hline 3.5 & 2.9 & -115.024984 & -115.034914 & -115.034046 & -115.033393 & -115.035149 \\
\hline 3.5 & 3.1 & -115.014913 & -115.024920 & -115.024015 & -115.023362 & -115.025144 \\
\hline 3.5 & 3.3 & -115.003499 & -115.013596 & -115.012644 & -115.011992 & -115.013814 \\
\hline 3.5 & 3.5 & -114.991443 & -115.001643 & -115.000632 & -114.999983 & -115.001861 \\
\hline 3.5 & 3.7 & -114.979272 & -114.989592 & -114.988507 & -114. & -114.989814 \\
\hline 3.5 & 3.9 & -114.967376 & -114.977832 & -114.976654 & -114.976011 & -114.978065 \\
\hline 3.5 & 4.1 & -114.956030 & -114.966646 & -114.965350 & -114.964713 & -114.966894 \\
\hline 3.5 & 4.5 & -114.935704 & -114.946723 & -114.945102 & -114.944481 & -114.947010 \\
\hline 3.5 & 4.7 & -114.926936 & -114.938208 & -114.936367 & -114.935757 & -114.938510 \\
\hline 3.5 & 5.0 & -114.915648 & -114.927379 & -114.925124 & -114.924532 & -114.927681 \\
\hline 3.5 & 5.2 & -114.909350 & -114.921446 & -114.918854 & -114.918275 & -114.921720 \\
\hline 3.5 & 5.5 & -114.901627 & -114.914358 & -114.911168 & -114.910607 & -114.914525 \\
\hline 3.5 & 6.0 & -114.892672 & -114.906672 & -114.902275 & -114.901737 & -114.906434 \\
\hline 3.5 & 8.0 & -114.881678 & -114.900949 & -114.891621 & -114.891072 & -114.897509 \\
\hline 3.7 & 1.8 & -114.947108 & -114.957141 & -114.956315 & -114.955656 & -114.957439 \\
\hline 3.7 & 1.9 & -114.970740 & -114.980771 & -114.979943 & -114.979284 & -114.981067 \\
\hline 3.7 & 2.0 & -114.988106 & -114.998139 & -114.997308 & -114.996649 & -114.998436 \\
\hline 3.7 & 2.2 & -115.008644 & -115.018699 & -115.017857 & -115.017198 & -115.018995 \\
\hline 3.7 & 2.4 & -115.015817 & -115.025908 & -115.025052 & -115.024392 & -115.026197 \\
\hline 3.7 & 2.5 & -115.015820 & -115.025939 & -115.025071 & -115.024412 & -115.026220 \\
\hline 3.7 & 2.52 & -115.015820 & -115.025939 & -115.025071 & -115.024412 & -115.026220 \\
\hline 3.7 & 2.6 & -115.014568 & -115.024708 & -115.023831 & -115.023171 & -115.024984 \\
\hline 3.7 & 2.7 & -115.011934 & -115.022104 & -115.021215 & -115.020555 & -115.022374 \\
\hline 3.7 & 2.9 & -115.004088 & -115.014330 & -115.013410 & -115.012750 & -115.014587 \\
\hline 3.7 & 3.1 & -114.993940 & -115.004268 & -115.003308 & -115.002649 & -115.004515 \\
\hline 3.7 & 3.3 & -114.982450 & -114.992878 & -114.991868 & -114.991210 & -114.993119 \\
\hline 3.7 & 3.5 & -114.970331 & -114.980874 & -114.979801 & -114.979144 & -114.981115 \\
\hline 3.7 & 3.7 & -114.958117 & -114.968789 & -114.967637 & -114.966984 & -114.969035 \\
\hline 3.7 & 3.9 & -114.946194 & -114.957016 & -114.955764 & -114.955116 & -114.957273 \\
\hline 3.7 & 4.1 & -114.934842 & -114.945836 & -114.944461 & -114.943819 & -114.946111 \\
\hline 3.7 & 4.5 & -114.914562 & -114.925989 & -114.924269 & -114.923645 & -114.926307 \\
\hline
\end{tabular}


Table S7. (continued)

\begin{tabular}{|c|c|c|c|c|c|c|}
\hline$R_{\mathrm{Be}-\mathrm{F}}$ & $R_{\mathrm{Be}-\mathrm{H}}$ & CCSD & $\operatorname{CCSD}(\mathrm{T})$ & R-CCSD $(\mathrm{T})$ & CR-CCSD $(\mathrm{T})$ & $\operatorname{MRCI}(\mathrm{Q})$ \\
\hline 3.7 & 4.7 & -114.905841 & -114.917541 & -114.915589 & -114.914976 & -114.917876 \\
\hline 3.7 & 5.0 & -114.894646 & -114.906837 & -114.904449 & -114.903855 & -114.907174 \\
\hline 3.7 & 5.2 & -114.888420 & -114.900999 & -114.898257 & -114.897677 & -114.901307 \\
\hline 3.7 & 5.5 & -114.880805 & -114.894058 & -114.890693 & -114.890132 & -114.894256 \\
\hline 3.7 & 6.0 & -114.872008 & -114.886597 & -114.881982 & -114.881446 & -114.886378 \\
\hline 3.7 & 8.0 & -114.861220 & -114.881279 & -114.871634 & -114.871096 & -114.877788 \\
\hline 3.9 & 1.8 & -114.927168 & -114.937469 & -114.936596 & -114.935933 & -114.937781 \\
\hline 3.9 & 1.9 & -114.950782 & -114.961082 & -114.960206 & -114.959544 & -114.961392 \\
\hline 3.9 & 2.0 & -114.968116 & -114.978422 & -114.977543 & -114.976880 & -114.978733 \\
\hline 3.9 & 2.2 & -114.988571 & -114.998905 & -114.998015 & -114.997352 & -114.999215 \\
\hline 3.9 & 2.4 & -114.995677 & -115.006056 & -115.005149 & -115.004486 & -115.006357 \\
\hline 3.9 & 2.5 & -114.995660 & -115.006074 & -115.005154 & -115.004491 & -115.006368 \\
\hline 3.9 & 2.52 & -114.995660 & -115.006074 & -115.005154 & -115.004491 & -115.006368 \\
\hline 3.9 & 2.6 & -114.994397 & -115.004837 & -115.003908 & -115.003244 & -115.005126 \\
\hline 3.9 & 2.7 & -114.991746 & -115.002222 & -115.001278 & -115.000614 & -115.002503 \\
\hline 3.9 & 2.9 & -114.983841 & -114.994399 & -114.993422 & -114.992758 & -114.994668 \\
\hline 3.9 & 3.1 & -114.973622 & -114.984276 & -114.983256 & -114.982593 & -114.984536 \\
\hline 3.9 & 3.3 & -114.962070 & -114.972836 & -114.971762 & -114.971100 & -114.973090 \\
\hline 3.9 & 3.5 & -114.949907 & -114.960798 & -114.959657 & -114. & -114.961052 \\
\hline 3.9 & 3.7 & -114.937666 & -114.948700 & -114.947475 & -114.946820 & -114.948960 \\
\hline 3.9 & 3.9 & -114.925736 & -114.936933 & -114.935603 & -114.934952 & -114.937205 \\
\hline 3.9 & 4.1 & -114.914394 & -114.925779 & -114.924317 & -114.923673 & -114.926069 \\
\hline 3.9 & 4.5 & -114.894183 & -114.906038 & -114.9 & -114.903586 & -114.906373 \\
\hline 3.9 & 4.7 & -114.885518 & -114.897665 & -114.895594 & -114.894982 & -114.898020 \\
\hline 3.9 & 5.0 & -114.874422 & -114.887096 & -114.884567 & -114.883975 & -114.887454 \\
\hline 3.9 & 5.2 & -114.868267 & -114.881356 & -114.878456 & -114.877879 & -114.881685 \\
\hline 3.9 & 5.5 & -114.860755 & -114.874561 & -114.871012 & -114.870454 & -114.874777 \\
\hline 3.9 & 6.0 & -114.852100 & -114.867318 & -114.862474 & -114.861942 & -114.867104 \\
\hline 3.9 & 8.0 & -114.841469 & -114.862415 & -114.852406 & -114.851881 & -114.858823 \\
\hline 4.1 & 1.8 & -114.908131 & -114.918705 & -114.917780 & -114.917116 & -114.919018 \\
\hline 4.1 & 1.9 & -114.931724 & -114.942299 & -114.941372 & -114.940708 & -114.942611 \\
\hline 4.1 & 2.0 & -114.949029 & -114.959613 & -114.958681 & -114.958017 & -114.959924 \\
\hline 4.1 & 2.2 & -114.969413 & -114.980034 & -114.979089 & -114.978425 & -114.980343 \\
\hline 4.1 & 2.4 & -114.976474 & -114.987151 & -114.986187 & -114.985523 & -114.987450 \\
\hline 4.1 & 2.5 & -114.976446 & -114.987164 & -114.986186 & -114.985521 & -114.987456 \\
\hline 4.1 & 2.52 & -114.976446 & -114.987164 & -114.986186 & -114.985521 & -114.987456 \\
\hline 4.1 & 2.6 & -114.975175 & -114.985924 & -114.984934 & -114.984270 & -114.986210 \\
\hline 4.1 & 2.7 & -114.972507 & -114.983297 & -114.982292 & -114.981627 & -114.983577 \\
\hline 4.1 & 2.9 & -114.964547 & -114.975432 & -114.974390 & -114.973725 & -114.975700 \\
\hline 4.1 & 3.1 & -114.954268 & -114.965263 & -114.964175 & -114.963511 & -114.965520 \\
\hline 4.1 & 3.3 & -114.942671 & -114.953790 & -114.952644 & -114.951982 & -114.954041 \\
\hline 4.1 & 3.5 & -114.930481 & -114.941740 & -114.940522 & -114.939862 & -114.941990 \\
\hline 4.1 & 3.7 & -114.918231 & -114.929648 & -114.928340 & -114.927684 & -114.929903 \\
\hline 4.1 & 3.9 & -114.906309 & -114.917905 & -114.916484 & -114.915835 & -114.918173 \\
\hline 4.1 & 4.1 & -114.894990 & -114.906792 & -114.905231 & -114.904589 & -114.907078 \\
\hline 4.1 & 4.5 & -114.874867 & -114.887179 & -114.885233 & -114.884612 & -114.887512 \\
\hline 4.1 & 4.7 & -114.866261 & -114.878890 & -114.876687 & -114.876078 & -114.879243 \\
\hline 4.1 & 5.0 & -114.855265 & -114.868462 & -114.865778 & -114.865190 & -114.868819 \\
\hline 4.1 & 5.2 & -114.849178 & -114.862819 & -114.859747 & -114.859175 & -114.863147 \\
\hline
\end{tabular}


Table S7. (continued)

\begin{tabular}{|c|c|c|c|c|c|c|}
\hline$R_{\mathrm{Be}-\mathrm{F}}$ & $R_{\mathrm{Be}-\mathrm{H}}$ & CCSD & $\operatorname{CCSD}(\mathrm{T})$ & R-CCSD $(\mathrm{T})$ & CR-CCSD $(\mathrm{T})$ & $\operatorname{MRCI}(\mathrm{Q})$ \\
\hline 4.1 & 5.5 & -114.841762 & -114.856169 & -114.852419 & -114.851868 & -114.856379 \\
\hline 4.1 & 6.0 & -114.833232 & -114.849136 & -114.844043 & -114.843519 & -114.848901 \\
\hline 4.1 & 8.0 & -114.822708 & -114.844655 & -114.834227 & -114.833715 & -114.840902 \\
\hline 4.5 & 1.8 & -114.873319 & -114.884491 & -114.883433 & -114.882774 & -114.884744 \\
\hline 4.5 & 1.9 & -114.896876 & -114.908055 & -114.906994 & -114.906334 & -114.908307 \\
\hline 4.5 & 2.0 & -114.914133 & -114.925329 & -114.924262 & -114.923602 & -114.925579 \\
\hline 4.5 & 2.2 & -114.934422 & -114.945676 & -114.944590 & -114.943930 & -114.945919 \\
\hline 4.5 & 2.4 & -114.941433 & -114.952769 & -114.951659 & -114.950998 & -114.953001 \\
\hline 4.5 & 2.5 & -114.941391 & -114.952787 & -114.951658 & -114.950996 & -114.953010 \\
\hline 4.5 & 2.52 & -114.941391 & -114.952787 & -114.951658 & -114.950996 & -114.953010 \\
\hline 4.5 & 2.6 & -114.940106 & -114.951545 & -114.950402 & -114.949740 & -114.951763 \\
\hline 4.5 & 2.7 & -114.937412 & -114.948908 & -114.947745 & -114.947083 & -114.949118 \\
\hline 4.5 & 2.9 & -114.929378 & -114.941002 & -114.939793 & -114.939132 & -114.941196 \\
\hline 4.5 & 3.1 & -114.919033 & -114.930801 & -114.929536 & -114.928875 & -114.930982 \\
\hline 4.5 & 3.3 & -114.907398 & -114.919329 & -114.917994 & -114.917335 & -114.919499 \\
\hline 4.5 & 3.5 & -114.895203 & -114.907314 & -114.905893 & -114.905237 & -114.907479 \\
\hline 4.5 & 3.7 & -114.882978 & -114.895289 & -114.893763 & -114.893112 & -114.895456 \\
\hline 4.5 & 3.9 & -114.871106 & -114 & -114.881987 & 31342 & -114 \\
\hline 4.5 & 4.1 & -114.859863 & -114.872653 & -114.870838 & -114.870201 & 372841 \\
\hline 4.5 & 4.5 & -114.839948 & -114.853359 & -114.851108 & -114.850495 & -114.853586 \\
\hline 4.5 & 4.7 & -114.831466 & -114.845256 & -114.842717 & -114.842118 & -114.845501 \\
\hline 4.5 & 5.0 & -114.820662 & -114 & -114.832050 & -114 . & -114 \\
\hline 4.5 & 5.2 & -114.814698 & -114.829679 & -114.826179 & -114.825620 & 29886 \\
\hline 4.5 & 5.5 & -114.807445 & -114.823314 & -114.819070 & -114.818534 & -114.823388 \\
\hline 4.5 & 6.0 & -114.799107 & -114.816690 & -114.810985 & -114.810478 & -114.816271 \\
\hline 4.5 & 8.0 & -114.788637 & 3098 & -114.801588 & -114.801095 & 08769 \\
\hline 4.7 & 1.8 & -114.857640 & -114.869167 & -114.868019 & -114.867363 & -114.869345 \\
\hline 4.7 & 1.9 & -114.881185 & -114.892722 & -114.891569 & -114.890913 & -114.892897 \\
\hline 4.7 & 2.0 & -114.898426 & -114.909985 & -114.908825 & -114.908169 & -114.910157 \\
\hline 4.7 & 2.2 & -114.918684 & 30315 & -114.929134 & -114.928477 & -114.930478 \\
\hline 4.7 & 2.4 & -114.925680 & -114.937411 & -114.936201 & -114.935542 & -114.937561 \\
\hline 4.7 & 2.5 & -114.925634 & -114.937437 & -114.936205 & -114.935545 & -114.937576 \\
\hline 4.7 & 2.52 & -114.925634 & -114.937437 & -114.936205 & -114.935545 & -114.937576 \\
\hline 4.7 & 2.6 & -114.924346 & -114.936201 & -114.934952 & -114.934292 & -114.936333 \\
\hline 4.7 & 2.7 & -114.921644 & -114.933568 & -114.932297 & -114.931636 & -114.933692 \\
\hline 4.7 & 2.9 & -114.913596 & -114.925672 & -114.924347 & -114.923687 & -114.925776 \\
\hline 4.7 & 3.1 & -114.903244 & -114.915490 & -114.914101 & -114.913441 & -114.915576 \\
\hline 4.7 & 3.3 & -114.891615 & -114.904051 & -114.902584 & -114.901925 & -114.904123 \\
\hline 4.7 & 3.5 & -114.879439 & -114.892083 & -114.890521 & -114.889865 & -114.892146 \\
\hline 4.7 & 3.7 & -114.867243 & -114.880119 & -114.878441 & -114.877790 & -114.880179 \\
\hline 4.7 & 3.9 & -114.855412 & -114.868546 & -114.866725 & -114.866081 & -114.868607 \\
\hline 4.7 & 4.1 & -114.844217 & -114.857640 & -114.855646 & -114.855010 & -114.857709 \\
\hline 4.7 & 4.5 & -114.824417 & -114.838538 & -114.836076 & -114.835464 & -114.838634 \\
\hline 4.7 & 4.7 & -114.815997 & -114.830541 & -114.827771 & -114.827174 & -114.830647 \\
\hline 4.7 & 5.0 & -114.805284 & -114.820571 & -114.817233 & -114.816660 & -114.820662 \\
\hline 4.7 & 5.2 & -114.799374 & -114.815234 & -114.811442 & -114.810886 & -114.815280 \\
\hline 4.7 & 5.5 & -114.792189 & -114.809020 & -114.804442 & -114.803909 & -114.808915 \\
\hline 4.7 & 6.0 & -114.783917 & -114.802610 & -114.796492 & -114.795989 & -114.801972 \\
\hline 4.7 & 8.0 & -114.773387 & -114.799527 & -114.787273 & -114.786779 & -114.794692 \\
\hline
\end{tabular}


Table S7. (continued)

\begin{tabular}{|c|c|c|c|c|c|c|}
\hline$R_{\mathrm{Be}-\mathrm{F}}$ & $R_{\mathrm{Be}-\mathrm{H}}$ & CCSD & $\operatorname{CCSD}(\mathrm{T})$ & $\mathrm{R}-\mathrm{CCSD}(\mathrm{T})$ & CR-CCSD(T) & MRCI(Q) \\
\hline 5.0 & 1.8 & -114.836246 & -114.848437 & -114.847103 & -114.846450 & -114.848421 \\
\hline 5.0 & 1.9 & -114.859777 & -114.871985 & -114.870646 & -114.869992 & -114.871965 \\
\hline 5.0 & 2.0 & -114.877003 & -114.889243 & -114.887894 & -114.887239 & -114.889216 \\
\hline 5.0 & 2.2 & -114.897233 & -114.909573 & -114.908195 & -114.907538 & -114.909531 \\
\hline 5.0 & 2.4 & -114.904215 & -114.916693 & -114.915275 & -114.914616 & -114.916631 \\
\hline 5.0 & 2.5 & -114.904167 & -114.916742 & -114.915294 & -114.914634 & -114.916667 \\
\hline 5.0 & 2.52 & -114.904167 & -114.916742 & -114.915294 & -114.914634 & -114.916667 \\
\hline 5.0 & 2.6 & -114.902879 & -114.915524 & -114.914054 & -114.913392 & -114.915439 \\
\hline 5.0 & 2.7 & -114.900177 & -114.912915 & -114.911416 & -114.910753 & -114.912818 \\
\hline 5.0 & 2.9 & -114.892134 & -114.905076 & -114.903508 & -114.902843 & -114.904952 \\
\hline 5.0 & 3.1 & -114.881801 & -114.894969 & -114.893319 & -114.892652 & -114.894817 \\
\hline 5.0 & 3.3 & -114.870207 & -114.883625 & -114.881876 & -114.881210 & -114.883449 \\
\hline 5.0 & 3.5 & -114.858080 & -114.871771 & -114.869906 & -114.869241 & -114.871575 \\
\hline 5.0 & 3.7 & -114.845948 & -114.859938 & -114.857933 & -114.857270 & -114.859725 \\
\hline 5.0 & 3.9 & -114.834192 & -114.848510 & -114.846337 & -114.845680 & -114.848285 \\
\hline 5.0 & 4.1 & -114.823082 & -114.837764 & -114.835388 & -114.834739 & -114.837530 \\
\hline 5.0 & 4.5 & -114.803470 & -114.819018 & -114.816103 & -114.815477 & -114.818771 \\
\hline 5.0 & 4.7 & -114.795145 & -114.811212 & -114.807946 & -114.807335 & -114.810950 \\
\hline 5.0 & 5.0 & -114.784564 & -114.801533 & -114.797626 & -114.797038 & -114.801214 \\
\hline 5.0 & 5.2 & -114.778730 & -114.796388 & -114.791972 & -114.791400 & -114.795989 \\
\hline 5.0 & 5.5 & -114.771634 & -114.790449 & -114.785153 & -114.784603 & -114.789840 \\
\hline 5.0 & 6.0 & -114.763435 & -114.784440 & -114.777432 & -114.776906 & -114.783171 \\
\hline 5.0 & 8.0 & -114.752706 & -114.782375 & -114.768514 & -114.767977 & -114.776227 \\
\hline 5.2 & 1.8 & -114.823332 & -114.836104 & -114.834595 & -114.833938 & -114.835888 \\
\hline 5.2 & 1.9 & -114.846859 & -114.859653 & -114.858137 & -114.857480 & -114.859431 \\
\hline 5.2 & 2.0 & -114.864079 & -114.876914 & -114.875386 & -114.874728 & -114.876684 \\
\hline 5.2 & 2.2 & -114.884301 & -114.897263 & -114.895698 & -114.895036 & -114.897011 \\
\hline 5.2 & 2.4 & -114.891282 & -114.904417 & -114.902801 & -114.902135 & -114.904140 \\
\hline 5.2 & 2.5 & -114.891237 & -114.904494 & -114.902840 & -114.902172 & -114.904200 \\
\hline 5.2 & 2.52 & -114.891237 & -114.904494 & -114.902840 & -114.902172 & -114.904200 \\
\hline 5.2 & 2.6 & -114.889952 & -114.903296 & -114.901616 & -114.900945 & -114.902991 \\
\hline 5.2 & 2.7 & -114.887258 & -114.900718 & -114.899000 & -114.898327 & -114.900397 \\
\hline 5.2 & 2.9 & -114.879234 & -114.892949 & -114.891146 & -114.890468 & -114.892596 \\
\hline 5.2 & 3.1 & -114.868930 & -114.882927 & -114.881023 & -114.880341 & -114.882540 \\
\hline 5.2 & 3.3 & -114.857375 & -114.871681 & -114.869658 & -114.868973 & -114.871261 \\
\hline 5.2 & 3.5 & -114.845296 & -114.859937 & -114.857774 & -114.857088 & -114.859489 \\
\hline 5.2 & 3.7 & -114.833219 & -114.848224 & -114.845897 & -114.845212 & -114.847749 \\
\hline 5.2 & 3.9 & -114.821524 & -114.836927 & -114.834404 & -114.833722 & -114.836424 \\
\hline 5.2 & 4.1 & -114.810480 & -114.826321 & -114.823565 & -114.822889 & -114.825794 \\
\hline 5.2 & 4.5 & -114.791006 & -114.807882 & -114.804516 & -114.803858 & -114.807304 \\
\hline 5.2 & 4.7 & -114.782748 & -114.800241 & -114.796481 & -114.795836 & -114.799621 \\
\hline 5.2 & 5.0 & -114.772259 & -114.790822 & -114.786342 & -114.785717 & -114.790087 \\
\hline 5.2 & 5.2 & -114.766478 & -114.785853 & -114.780805 & -114.780193 & -114.784990 \\
\hline 5.2 & 5.5 & -114.759440 & -114.780176 & -114.774148 & -114.773551 & -114.779013 \\
\hline 5.2 & 6.0 & -114.751285 & -114.774573 & -114.766641 & -114.766058 & -114.772567 \\
\hline 5.2 & 8.0 & -114.740378 & -114.773631 & -114.758082 & -114.757444 & -114.765887 \\
\hline 5.5 & 1.8 & -114.805850 & -114.819825 & -114.817929 & -114.817249 & -114.819188 \\
\hline 5.5 & 1.9 & -114.829375 & -114.843384 & -114.841478 & -114.840797 & -114.842737 \\
\hline 5.5 & 2.0 & -114.846595 & -114.860665 & -114.858740 & -114.858057 & -114.860004 \\
\hline
\end{tabular}


Table S7. (continued)

\begin{tabular}{|c|c|c|c|c|c|c|}
\hline$R_{\mathrm{Be}-\mathrm{F}}$ & $R_{\mathrm{Be}-\mathrm{H}}$ & CCSD & $\mathrm{CCSD}(\mathrm{T})$ & $\mathrm{R}-\mathrm{CCSD}(\mathrm{T})$ & CR-CCSD $(\mathrm{T})$ & $\operatorname{MRCI}(\mathrm{Q})$ \\
\hline 5.5 & 2.2 & -114.866820 & -114.881076 & -114.879095 & -114.878405 & -114.880386 \\
\hline 5.5 & 2.4 & -114.873815 & -114.888323 & -114.886263 & -114.885564 & -114.887603 \\
\hline 5.5 & 2.5 & -114.873785 & -114.888470 & -114.886354 & -114.885648 & -114.887735 \\
\hline 5.5 & 2.52 & -114.873785 & -114.888470 & -114.886354 & -114.885648 & -114.887735 \\
\hline 5.5 & 2.6 & -114.872513 & -114.887326 & -114.885169 & -114.884458 & -114.886578 \\
\hline 5.5 & 2.7 & -114.869839 & -114.884821 & -114.882608 & -114.881891 & -114.884057 \\
\hline 5.5 & 2.9 & -114.861868 & -114.877221 & -114.874880 & -114.874151 & -114.876425 \\
\hline 5.5 & 3.1 & -114.851632 & -114.867392 & -114.864904 & -114.864162 & -114.866564 \\
\hline 5.5 & 3.3 & -114.840157 & -114.856360 & -114.853702 & -114.852949 & -114.855507 \\
\hline 5.5 & 3.5 & -114.828169 & -114.844849 & -114.841997 & -114.841233 & -114.843967 \\
\hline 5.5 & 3.7 & -114.816193 & -114.833389 & -114.830310 & -114.829538 & -114.832483 \\
\hline 5.5 & 3.9 & -114.804607 & -114.822362 & -114.819020 & -114.818243 & -114.821436 \\
\hline 5.5 & 4.1 & -114.793678 & -114.812046 & -114.808396 & -114.807616 & -114.811105 \\
\hline 5.5 & 4.5 & -114.774442 & -114.794257 & -114.789811 & -114.789032 & -114.793284 \\
\hline 5.5 & 4.7 & -114.766302 & -114.786981 & -114.782026 & -114.781248 & -114.785972 \\
\hline 5.5 & 5.0 & -114.755979 & -114.778166 & -114.772281 & -114.771503 & -114.777034 \\
\hline 5.5 & 5.2 & -114.750295 & -114.773634 & -114.767013 & -114.766231 & -114.772336 \\
\hline 5.5 & 5.5 & -114.743381 & -114.768651 & -114.760755 & -114.759962 & -114.766938 \\
\hline 5.5 & 6.0 & -114.735350 & -114.764238 & -114.753859 & -114.753024 & -114.761356 \\
\hline 5.5 & 8.0 & -114.724319 & -114.767135 & -114.746790 & -114.745668 & -114.755924 \\
\hline 6.0 & 1.8 & -114.781318 & -114.798975 & -114.795763 & -114.794909 & -114.797427 \\
\hline 6.0 & 1.9 & -114.804851 & -114.822580 & -114.819342 & -114.818484 & -114.821014 \\
\hline 6.0 & 2.0 & -114.822086 & -114.839941 & -114.836660 & -114.835794 & -114.838347 \\
\hline 6.0 & 2.2 & -114.842363 & -114.860601 & -114.857186 & -114.856294 & -114.858914 \\
\hline 6.0 & 2.4 & -114.849434 & -114.868194 & -114.864593 & -114.863664 & -114.866367 \\
\hline 6.0 & 2.5 & -114.849463 & -114.868591 & -114.864856 & -114.863901 & -114.866655 \\
\hline 6.0 & 2.52 & -114.849463 & -114.868591 & -114.864856 & -114.863901 & -114.866655 \\
\hline 6.0 & 2.6 & -114.848236 & -114.867629 & -114.863796 & -114.862822 & -114.865611 \\
\hline 6.0 & 2.7 & -114.845625 & -114.865369 & -114.861405 & -114.860405 & -114.863240 \\
\hline 6.0 & 2.9 & -114.837801 & -114.858311 & -114.854052 & -114.852998 & -114.855935 \\
\hline 6.0 & 3.1 & -114.827735 & -114.849084 & -114.844489 & -114.843377 & -114.846440 \\
\hline 6.0 & 3.3 & -114.816450 & -114.838704 & -114.833733 & -114.832561 & -114.835790 \\
\hline 6.0 & 3.5 & -114.804670 & -114.827894 & -114.822500 & -114.821269 & -114.824722 \\
\hline 6.0 & 3.7 & -114.792917 & -114.817184 & -114.811314 & -114.810023 & -114.813780 \\
\hline 6.0 & 3.9 & -114.781567 & -114.806968 & -114.800555 & -114.799207 & -114.803368 \\
\hline 6.0 & 4.1 & -114.770889 & -114.797545 & -114.790503 & -114.789095 & -114.793798 \\
\hline 6.0 & 4.5 & -114.752199 & -114.781927 & -114.773248 & -114.771705 & -114.778040 \\
\hline 6.0 & 4.7 & -114.744360 & -114.776027 & -114.766264 & -114.764634 & -114.772112 \\
\hline 6.0 & 5.0 & -114.734531 & -114.769790 & -114.757943 & -114.756139 & -114.765672 \\
\hline 6.0 & 5.2 & -114.729208 & -114.767380 & -114.753786 & -114.751827 & -114.762799 \\
\hline 6.0 & 5.5 & -114.722875 & -114.766216 & -114.749415 & -114.747163 & -114.760037 \\
\hline 6.0 & 6.0 & -114.715872 & -114.769598 & -114.746015 & -114.743127 & -114.757762 \\
\hline 6.0 & 8.0 & -114.708187 & -114.804606 & -114.750579 & -114.744735 & -114.756057 \\
\hline
\end{tabular}


Table S8. The CCSD, CCSD(T), R-CCSD(T), CR-CCSD(T), and MRCI(Q) energies, in hartree, of the BeFH system, as described by the cc-pVTZ basis set, for the Be-F-H angle $\theta=0^{\circ}$ corresponding to the Be atom approaching the $\mathrm{HF}$ molecule from the $\mathrm{H}$ side. The $\mathrm{Be}-\mathrm{H}$ and $\mathrm{H}-\mathrm{F}$ bond distances, $R_{\mathrm{Be}-\mathrm{H}}$ and $R_{\mathrm{H}-\mathrm{F}}$, respectively, are in bohr.

\begin{tabular}{|c|c|c|c|c|c|c|}
\hline$R_{\mathrm{Be}-\mathrm{H}}$ & $R_{\mathrm{H}-\mathrm{F}}$ & CCSD & $\operatorname{CCSD}(\mathrm{T})$ & R-CCSD $(\mathrm{T})$ & CR-CCSD(T) & $\mathrm{MRCI}(\mathrm{Q})$ \\
\hline 1.8 & 1.2 & -114.556429 & -114.565527 & -114.564347 & -114.563871 & -114.562983 \\
\hline 1.8 & 1.4 & -114.699239 & -114.709417 & -114.708043 & -114.707503 & -114.707759 \\
\hline 1.8 & 1.6 & -114.754613 & -114.766336 & -114.764714 & -114.764089 & -114.764017 \\
\hline 1.8 & 1.7325 & -114.769441 & -114.782485 & -114.780663 & -114.779967 & -114.780717 \\
\hline 1.8 & 1.8 & -114.773570 & -114.787357 & -114.785422 & -114.784686 & -114.785893 \\
\hline 1.8 & 2.0 & -114.778751 & -114.794810 & -114.792515 & -114.791665 & -114.794209 \\
\hline 1.8 & 2.25 & -114.777939 & -114.796681 & -114.793834 & -114.792876 & -114.796752 \\
\hline 1.8 & 2.5 & -114.773021 & -114.794585 & -114.790874 & -114.789790 & -114.794449 \\
\hline 1.8 & 2.75 & -114.765837 & -114.791070 & -114.785911 & -114.784630 & -114.789440 \\
\hline 1.8 & 3.0 & -114.757769 & -114.788454 & -114.780836 & -114.779236 & -114.783313 \\
\hline 1.8 & 3.5 & -114.742552 & -114.798239 & -114.777495 & -114.773833 & -114.772554 \\
\hline 1.8 & 4.0 & -114.731188 & -114.869136 & -114.796035 & -114.782938 & -114.767214 \\
\hline 1.9 & 1.2 & -114.584832 & -114.593892 & -114.592715 & -114.592240 & -114.591336 \\
\hline 1.9 & 1.4 & -114.729230 & -114.739370 & -114.738009 & -114.737468 & -114.737654 \\
\hline 1.9 & 1.6 & -114.785957 & -114.797600 & -114.796009 & -114.795383 & -114.795426 \\
\hline 1.9 & 1.7325 & -114.801246 & -114.814131 & -114.812356 & -114.811662 & -114.812456 \\
\hline 1.9 & 1.8 & -114.805428 & -114.818997 & -114.817121 & -114.816390 & -114.817595 \\
\hline 1.9 & 2.0 & -114.810083 & -114.825725 & -114.823521 & -114.822688 & -114.825092 \\
\hline 1.9 & 2.25 & -114.807807 & -114.825928 & -114.823214 & -114.822284 & -114.825927 \\
\hline 1.9 & 2.5 & -114.801423 & -114.822168 & -114.818669 & -114.817628 & -114.822058 \\
\hline 1.9 & 2.75 & -114.793095 & -114.817224 & -114.812427 & -114.811212 & -114.815906 \\
\hline 1.9 & 3.0 & -114.784172 & -114.813311 & -114.806309 & -114.804802 & -114.808993 \\
\hline 1.9 & 3.5 & -114.767820 & -114.819684 & -114.800993 & -114.797577 & -114.797159 \\
\hline 1.9 & 4.0 & -114.755796 & -114.880739 & -114.816348 & -114.804406 & -114.791279 \\
\hline 2.0 & 1.2 & -114.609026 & -114.618029 & -114.616861 & -114.616387 & -114.615470 \\
\hline 2.0 & 1.4 & -114.754688 & -114.764752 & -114.763411 & -114.762872 & -114.762980 \\
\hline 2.0 & 1.6 & -114.812288 & -114.823794 & -114.822240 & -114.821616 & -114.821731 \\
\hline 2.0 & 1.7325 & -114.827712 & -114.840373 & -114.838652 & -114.837964 & -114.838763 \\
\hline 2.0 & 1.8 & -114.831800 & -114.845091 & -114.843277 & -114.842556 & -114.843726 \\
\hline 2.0 & 2.0 & -114.835624 & -114.850815 & -114.848702 & -114.847889 & -114.850147 \\
\hline 2.0 & 2.25 & -114.831728 & -114.849227 & -114.846644 & -114.845741 & -114.849159 \\
\hline 2.0 & 2.5 & -114.823815 & -114.843769 & -114.840471 & -114.839469 & -114.843684 \\
\hline 2.0 & 2.75 & -114.814297 & -114.837392 & -114.832924 & -114.831767 & -114.836353 \\
\hline 2.0 & 3.0 & -114.804482 & -114.832206 & -114.825752 & -114.824325 & -114.828629 \\
\hline 2.0 & 3.5 & -114.786949 & -114.835432 & -114.818516 & -114.815318 & -114.815686 \\
\hline 2.0 & 4.0 & -114.774244 & -114.888117 & -114.831011 & -114.820075 & -114.809233 \\
\hline 2.2 & 1.2 & -114.647917 & -114.656736 & -114.655601 & -114.655134 & -114.654801 \\
\hline 2.2 & 1.4 & -114.795081 & -114.804892 & -114.803606 & -114.803075 & -114.803011 \\
\hline 2.2 & 1.6 & -114.853247 & -114.864345 & -114.862876 & -114.862267 & -114.862435 \\
\hline 2.2 & 1.7325 & -114.868250 & -114.880341 & -114.878732 & -114.878067 & -114.878797 \\
\hline 2.2 & 1.8 & -114.871868 & -114.884495 & -114.882808 & -114.882114 & -114.883152 \\
\hline 2.2 & 2.0 & -114.873502 & -114.887758 & -114.885816 & -114.885045 & -114.887005 \\
\hline 2.2 & 2.25 & -114.866148 & -114.882445 & -114.880101 & -114.879251 & -114.882250 \\
\hline 2.2 & 2.5 & -114.855088 & -114.873575 & -114.870633 & -114.869701 & -114.873513 \\
\hline 2.2 & 2.75 & -114.843106 & -114.864347 & -114.860446 & -114.859385 & -114.863743 \\
\hline 2.2 & 3.0 & -114.831435 & -114.856677 & -114.851149 & -114.849854 & -114.854328 \\
\hline
\end{tabular}


Table S8. (continued)

\begin{tabular}{|c|c|c|c|c|c|c|}
\hline$R_{\mathrm{Be}-\mathrm{H}}$ & $R_{\mathrm{H}-\mathrm{F}}$ & CCSD & $\operatorname{CCSD}(\mathrm{T})$ & $\mathrm{R}-\mathrm{CCSD}(\mathrm{T})$ & CR-CCSD $(\mathrm{T})$ & MRCI(Q) \\
\hline 2.2 & 3.5 & -114.811441 & -114.854168 & -114.840181 & -114.837367 & -114.839104 \\
\hline 2.2 & 4.0 & -114.797316 & -114.893178 & -114.847631 & -114.838407 & -114.831404 \\
\hline 2.4 & 1.2 & -114.677438 & -114.685991 & -114.684902 & -114.684446 & -114.683484 \\
\hline 2.4 & 1.4 & -114.824957 & -114.834411 & -114.833191 & -114.832674 & -114.832424 \\
\hline 2.4 & 1.6 & -114.882611 & -114.893199 & -114.891820 & -114.891234 & -114.891377 \\
\hline 2.4 & 1.7325 & -114.896666 & -114.908116 & -114.906615 & -114.905980 & -114.906603 \\
\hline 2.4 & 1.8 & -114.899623 & -114.911537 & -114.909969 & -114.909310 & -114.910192 \\
\hline 2.4 & 2.0 & -114.898780 & -114.912125 & -114.910336 & -114.909609 & -114.911283 \\
\hline 2.4 & 2.25 & -114.887934 & -114.903125 & -114.900986 & -114.900187 & -114.902806 \\
\hline 2.4 & 2.5 & -114.873734 & -114.890923 & -114.888279 & -114.887405 & -114.890835 \\
\hline 2.4 & 2.75 & -114.859261 & -114.878915 & -114.875472 & -114.874487 & -114.878581 \\
\hline 2.4 & 3.0 & -114.845696 & -114.868853 & -114.864065 & -114.862877 & -114.867390 \\
\hline 2.4 & 3.5 & -114.823186 & -114.861175 & -114.849505 & -114.847026 & -114.849890 \\
\hline 2.4 & 4.0 & -114.807592 & -114.889264 & -114.852523 & -114.844738 & -114.840822 \\
\hline 2.5 & 1.2 & -114.689476 & -114.697875 & -114 & 6362 & -114 \\
\hline 2.5 & 1.4 & -114.836870 & -114.846123 & -114.844936 & -114.844429 & -114.844086 \\
\hline 2.5 & 1.6 & -114.894035 & -114.904355 & -114.903019 & -114.902446 & -114.902567 \\
\hline 2.5 & 1.7325 & 07527 & -114 & 17204 & 6586 & -114 \\
\hline 2.5 & 1.8 & 10130 & 21693 & 0180 & 19538 & 20341 \\
\hline 2.5 & 2.0 & -114.908046 & -114.920960 & -114.919239 & -114.918533 & -114.920074 \\
\hline 2.5 & 2.25 & -114.895513 & -114.910195 & -114.908147 & -114.907371 & -114.909810 \\
\hline 2.5 & 2.5 & -114 . & 6398 & -114 & -114. & -114.896282 \\
\hline 2.5 & 2.75 & 54100 & -114.883051 & -114 & 8850 & -114.882794 \\
\hline 2.5 & 3.0 & -114.849597 & -114.871842 & -114.867366 & -114.866224 & -114.870701 \\
\hline 2.5 & 3.5 & -114.825841 & -114.861770 & -114.851078 & -114.848751 & -114.852094 \\
\hline 2.5 & 4.0 & -114 & -114 & 2055 & -114 & -114 \\
\hline 2.52 & 1.2 & -114.691695 & -114.700061 & -114.699003 & -114.698555 & -114.697589 \\
\hline 2.52 & 1.4 & -114.839046 & -114.848258 & -114.847077 & -114.846572 & -114.846212 \\
\hline 2.52 & 1.6 & -114.896103 & -114.906368 & -114.905040 & -114.904470 & -114.904586 \\
\hline 2.52 & 1.7325 & -114. & 20543 & -114. & -114 & -114.919038 \\
\hline 2.52 & 1.8 & -114.912011 & -114.923505 & -114.922003 & -114.921364 & -114.922151 \\
\hline 2.52 & 2.0 & -114.909682 & -114.922513 & -114.920805 & -114.920103 & -114.921619 \\
\hline 2.52 & 2.25 & -114.896821 & -114.911405 & -114.909374 & -114.908602 & -114.911008 \\
\hline 2.52 & 2.5 & -114 & -114.897296 & -114.894804 & -114.893961 & -114.897174 \\
\hline 2.52 & 2.75 & -114.864870 & -114.883687 & -114.880475 & -114.879528 & -114.883442 \\
\hline 2.52 & 3.0 & -114.850182 & -114.872253 & -114.867835 & -114.866702 & -114.871167 \\
\hline 2.52 & 3.5 & -114.826177 & -114.861717 & -114.851208 & -114.848910 & -114.852342 \\
\hline 2.52 & 4.0 & -114.809695 & -114.884198 & -114.851788 & -114.844762 & -114.842407 \\
\hline 2.6 & 1.2 & -114.699990 & -114.708224 & -114.707186 & -114.706745 & -114.705782 \\
\hline 2.6 & 1.4 & -114.847126 & -114.856172 & -114.855018 & -114.854521 & -114.854092 \\
\hline 2.6 & 1.6 & -114.903724 & -114.913775 & -114.912480 & -114.911921 & -114.912014 \\
\hline 2.6 & 1.7325 & -114.916637 & -114.927449 & -114.926046 & -114.925444 & -114.925946 \\
\hline 2.6 & 1.8 & -114.918888 & -114.930111 & -114.928649 & -114.928025 & -114.928750 \\
\hline 2.6 & 2.0 & -114.915604 & -114.928108 & -114.926450 & -114.925764 & -114.927174 \\
\hline 2.6 & 2.25 & -114.901464 & -114.915666 & -114.913700 & -114.912946 & -114.915220 \\
\hline 2.6 & 2.5 & -114.884285 & -114.900342 & -114.897941 & -114.897117 & -114.900190 \\
\hline 2.6 & 2.75 & -114.867399 & -114.885703 & -114.882627 & -114.881704 & -114.885495 \\
\hline 2.6 & 3.0 & -114.851975 & -114.873383 & -114.869186 & -114.868086 & -114.872493 \\
\hline 2.6 & 3.5 & -114.826982 & -114.861035 & -114.851218 & -114.849032 & -114.852799 \\
\hline
\end{tabular}


Table S8. (continued)

\begin{tabular}{|c|c|c|c|c|c|c|}
\hline$R_{\mathrm{Be}-\mathrm{H}}$ & $R_{\mathrm{H}-\mathrm{F}}$ & CCSD & $\operatorname{CCSD}(\mathrm{T})$ & $\mathrm{R}-\mathrm{CCSD}(\mathrm{T})$ & CR-CCSD(T) & $\operatorname{MRCI}(\mathrm{Q})$ \\
\hline 2.6 & 4.0 & -114.809905 & -114.880095 & -114.850249 & -114.843688 & -114.842265 \\
\hline 2.7 & 1.2 & -114.709163 & -114.717228 & -114.716216 & -114.715782 & -114.714838 \\
\hline 2.7 & 1.4 & -114.855960 & -114.864798 & -114.863675 & -114.863189 & -114.862679 \\
\hline 2.7 & 1.6 & -114.911955 & -114.921744 & -114.920488 & -114.919942 & -114.920006 \\
\hline 2.7 & 1.7325 & -114.924299 & -114.934807 & -114.933449 & -114.932862 & -114.933305 \\
\hline 2.7 & 1.8 & -114.926213 & -114.937109 & -114.935695 & -114.935088 & -114.935731 \\
\hline 2.7 & 2.0 & -114.921796 & -114.933913 & -114.932313 & -114.931646 & -114.932938 \\
\hline 2.7 & 2.25 & -114.906147 & -114.919900 & -114.918009 & -114.917275 & -114.919392 \\
\hline 2.7 & 2.5 & -114.887577 & -114.903126 & -114.900828 & -114.900027 & -114.902929 \\
\hline 2.7 & 2.75 & -114.869535 & -114.887242 & -114.884321 & -114.883426 & -114.887059 \\
\hline 2.7 & 3.0 & -114.853205 & -114.873849 & -114.869901 & -114.868839 & -114.873147 \\
\hline 2.7 & 3.5 & -114.826980 & -114.859327 & -114.850291 & -114.848236 & -114.852373 \\
\hline 2.7 & 4.0 & -114.809151 & -114.874418 & -114.847455 & -114.841434 & -114.841060 \\
\hline 2.9 & 1.2 & -114.724195 & -114.731925 & -114.730961 & -114.730544 & -114.729583 \\
\hline 2.9 & 1.4 & -114.870223 & -114.878657 & -114.877592 & -114.877126 & -114.876479 \\
\hline 2.9 & 1.6 & -114.925037 & -114.934331 & -114.933144 & -114.932625 & -114.932615 \\
\hline 2.9 & 1.7325 & -114.936332 & -114.946274 & -114.944995 & -114.944438 & -114.944271 \\
\hline 2.9 & 1.8 & -114.937637 & -114.947932 & -114.946602 & -114.946026 & -114.946039 \\
\hline 2.9 & 2.0 & -114.931201 & -114.942611 & -114.941112 & -114.940481 & -114.941554 \\
\hline 2.9 & 2.25 & -114.912832 & -114.925772 & -114.924010 & -114.923315 & -114.925140 \\
\hline 2.9 & 2.5 & -114.891684 & -114.906320 & -114.904199 & -114.903438 & -114.906015 \\
\hline 2.9 & 2.75 & -114.871437 & -114.888088 & -114.885427 & -114.884580 & -114.887893 \\
\hline 2.9 & 3.0 & -114.853336 & -114.872645 & -114.869114 & -114.868121 & -114.872169 \\
\hline 2.9 & 3.5 & -114.824634 & -114.854036 & -114.846315 & -114.844488 & -114.849197 \\
\hline 2.9 & 4.0 & -114.805251 & -114.862069 & -114.839941 & -114.834859 & -114.836218 \\
\hline 3.1 & 1.2 & -114.735845 & -114.743258 & -114.742338 & -114.741937 & -114.740953 \\
\hline 3.1 & 1.4 & -114.881111 & -114.889175 & -114.888160 & -114.887714 & -114.886959 \\
\hline 3.1 & 1.6 & -114.934851 & -114.943702 & -114.942575 & -114.942079 & -114.941999 \\
\hline 3.1 & 1.7325 & -114.945232 & -114.954676 & -114.953464 & -114.952933 & -114.952542 \\
\hline 3.1 & 1.8 & -114.946014 & -114.955780 & -114.954521 & -114.953973 & -114.953485 \\
\hline 3.1 & 2.0 & -114.937845 & -114.948643 & -114.947227 & -114.946628 & -114.947505 \\
\hline 3.1 & 2.25 & -114.917109 & -114.929344 & -114.927687 & -114.927027 & -114.928589 \\
\hline 3.1 & 2.5 & -114.893649 & -114.907499 & -114.905519 & -114.904795 & -114.907070 \\
\hline 3.1 & 2.75 & -114.871363 & -114.887114 & -114.884660 & -114.883854 & -114.886855 \\
\hline 3.1 & 3.0 & -114.851565 & -114.869762 & -114.866559 & -114.865623 & -114.869380 \\
\hline 3.1 & 3.5 & -114.820386 & -114.847403 & -114.840710 & -114.839070 & -114.844064 \\
\hline 3.1 & 4.0 & -114.799392 & -114.849389 & -114.831048 & -114.826729 & -114.829356 \\
\hline 3.3 & 1.2 & -114.744929 & -114.752059 & -114.751175 & -114.750789 & -114.749781 \\
\hline 3.3 & 1.4 & -114.889492 & -114.897228 & -114.896257 & -114.895828 & -114.894993 \\
\hline 3.3 & 1.6 & -114.942274 & -114.950739 & -114.949662 & -114.949187 & -114.949036 \\
\hline 3.3 & 1.7325 & -114.951861 & -114.960875 & -114.959718 & -114.959211 & -114.958548 \\
\hline 3.3 & 1.8 & -114.952193 & -114.961505 & -114.960305 & -114.959781 & -114.959180 \\
\hline 3.3 & 2.0 & -114.942544 & -114.952816 & -114.951469 & -114.950898 & -114.950541 \\
\hline 3.3 & 2.25 & -114.919778 & -114.931404 & -114.929833 & -114.929203 & -114.930529 \\
\hline 3.3 & 2.5 & -114.894298 & -114.907466 & -114.905599 & -114.904907 & -114.906910 \\
\hline 3.3 & 2.75 & -114.870172 & -114.885155 & -114.882861 & -114.882094 & -114.884804 \\
\hline 3.3 & 3.0 & -114.848792 & -114.866062 & -114.863112 & -114.862226 & -114.865697 \\
\hline 3.3 & 3.5 & -114.815203 & -114.840320 & -114.834417 & -114.832925 & -114.833565 \\
\hline
\end{tabular}


Table S8. (continued)

\begin{tabular}{|c|c|c|c|c|c|c|}
\hline$R_{\mathrm{Be}-\mathrm{H}}$ & $R_{\mathrm{H}-\mathrm{F}}$ & CCSD & $\operatorname{CCSD}(\mathrm{T})$ & R-CCSD $(\mathrm{T})$ & CR-CCSD(T) & MRCI(Q) \\
\hline 3.3 & 4.0 & -114.792582 & -114.837175 & -114.821752 & -114.818030 & -114.821492 \\
\hline 3.5 & 1.2 & -114.751930 & -114.758812 & -114.757960 & -114.757587 & -114.756555 \\
\hline 3.5 & 1.4 & -114.895872 & -114.903325 & -114.902390 & -114.901977 & -114.901084 \\
\hline 3.5 & 1.6 & -114.947827 & -114.955961 & -114.954927 & -114.954469 & -114.954251 \\
\hline 3.5 & 1.7325 & -114.956744 & -114.965389 & -114.964279 & -114.963791 & -114.963054 \\
\hline 3.5 & 1.8 & -114.956699 & -114.965622 & -114.964470 & -114.963967 & -114.963288 \\
\hline 3.5 & 2.0 & -114.945815 & -114.955636 & -114.954347 & -114.953799 & -114.953322 \\
\hline 3.5 & 2.25 & -114.921350 & -114.932452 & -114.930951 & -114.930347 & -114.930184 \\
\hline 3.5 & 2.5 & -114.894149 & -114.906727 & -114.904950 & -114.904287 & -114.906043 \\
\hline 3.5 & 2.75 & -114.868409 & -114.882730 & -114.880563 & -114.879829 & -114.882276 \\
\hline 3.5 & 3.0 & -114.845596 & -114.862087 & -114.859332 & -114.858489 & -114.861699 \\
\hline 3.5 & 3.5 & -114.809735 & -114.833360 & -114.828051 & -114.826675 & -114.827250 \\
\hline 3.5 & 4.0 & -114.785521 & -114.825943 & -114.812714 & -114.809446 & -114.813359 \\
\hline 3.7 & 1.2 & -114.757242 & -114.763911 & -114.763086 & -114.762725 & -114.761737 \\
\hline 3.7 & 1.4 & -114.900660 & -114.907870 & -114.906965 & 06565 & -114 \\
\hline 3.7 & 1.6 & -114.951927 & -114.959779 & -114.958779 & -114.958337 & -114.957464 \\
\hline 3.7 & 1.7325 & -114.960292 & -114.968624 & -114.967552 & -114.967081 & -114.966296 \\
\hline 3.7 & 1.8 & -114.959938 & -114 & -114 & -114.966935 & -114.966201 \\
\hline 3.7 & 2.0 & -114.948048 & 57486 & 56243 & 55715 & -114 \\
\hline 3.7 & 2.25 & -114.922191 & -114.932841 & -114.931399 & -114.930817 & -114.930521 \\
\hline 3.7 & 2.5 & -114.893555 & -114.905619 & -114.903915 & -114.903278 & -114.903252 \\
\hline 3.7 & 2.75 & -114 . & -114 & -114 & -114 & -114 \\
\hline 3.7 & 3.0 & -114.842359 & -114 . & 55583 & 354776 & -114.855035 \\
\hline 3.7 & 3.5 & -114.804429 & -114.826897 & -114.822021 & -114.820734 & -114.821283 \\
\hline 3.7 & 4.0 & -114.778716 & -114.816014 & -114.804384 & -114.801448 & -114.803407 \\
\hline 3.9 & 1.2 & -114.761220 & -114 & 66902 & -114.766551 & -114 \\
\hline 3.9 & 1.4 & -114.904206 & -114 . & -114.910330 & -114.909941 & -114.908987 \\
\hline 3.9 & 1.6 & -114.954914 & -114.962527 & -114.961556 & -114.961127 & -114.960228 \\
\hline 3.9 & 1.7325 & -114.962835 & -114.970902 & -114.969862 & -114.969405 & -114.968587 \\
\hline 3.9 & 1.8 & -114.962233 & -114.970547 & -114.969470 & -114.968999 & -114.968229 \\
\hline 3.9 & 2.0 & -114.949536 & -114.958648 & -114.957444 & -114.956932 & -114.956325 \\
\hline 3.9 & 2.25 & -114.922557 & -114.932819 & -114.931424 & -114.930861 & -114.930486 \\
\hline 3.9 & 2.5 & -114.892746 & -114.904364 & -114.902719 & -114.902102 & -114.901952 \\
\hline 3.9 & 2.75 & -114.864471 & -114.877716 & -114.875726 & -114.875044 & -114.875060 \\
\hline 3.9 & 3.0 & -114.839318 & -114.854586 & -114.852088 & -114.851311 & -114.851398 \\
\hline 3.9 & 3.5 & -114.799575 & -114.821150 & -114.816579 & -114.815358 & -114.815896 \\
\hline 3.9 & 4.0 & -114.772519 & -114.807553 & -114.797041 & -114.794341 & -114.796491 \\
\hline 4.1 & 1.2 & -114.764161 & -114.770491 & -114.769707 & -114.769363 & -114.768349 \\
\hline 4.1 & 1.4 & -114.906801 & -114.913629 & -114.912771 & -114.912391 & -114.911422 \\
\hline 4.1 & 1.6 & -114.957063 & -114.964474 & -114.963527 & -114.963108 & -114.962192 \\
\hline 4.1 & 1.7325 & -114.964630 & -114.972473 & -114.971460 & -114.971015 & -114.970175 \\
\hline 4.1 & 1.8 & -114.963832 & -114.971910 & -114.970860 & -114.970402 & -114.969607 \\
\hline 4.1 & 2.0 & -114.950494 & -114.959330 & -114.958159 & -114.957660 & -114.957020 \\
\hline 4.1 & 2.25 & -114.922621 & -114.932552 & -114.931196 & -114.930649 & -114.930224 \\
\hline 4.1 & 2.5 & -114.891863 & -114.903095 & -114.901497 & -114.900899 & -114.900678 \\
\hline 4.1 & 2.75 & -114.862645 & -114.875453 & -114.873523 & -114.872861 & -114.872788 \\
\hline 4.1 & 3.0 & -114.836599 & -114.851380 & -114.848964 & -114.848211 & -114.848223 \\
\hline 4.1 & 3.5 & -114.795334 & -114.816219 & -114.811852 & -114.810678 & -114.811246 \\
\hline 4.1 & 4.0 & -114.767150 & -114.800605 & -114.790826 & -114.788284 & -114.790518 \\
\hline
\end{tabular}


Table S8. (continued)

\begin{tabular}{|c|c|c|c|c|c|c|}
\hline$R_{\mathrm{Be}-\mathrm{H}}$ & $R_{\mathrm{H}-\mathrm{F}}$ & CCSD & $\operatorname{CCSD}(\mathrm{T})$ & R-CCSD $(\mathrm{T})$ & CR-CCSD(T) & MRCI(Q) \\
\hline 4.5 & 1.2 & -114.767856 & -114.773943 & -114.773188 & -114.772858 & -114.771840 \\
\hline 4.5 & 1.4 & -114.910004 & -114.916560 & -114.915734 & -114.915369 & -114.914393 \\
\hline 4.5 & 1.6 & -114.959637 & -114.966735 & -114.965826 & -114.965423 & -114.964491 \\
\hline 4.5 & 1.7325 & -114.966710 & -114.974207 & -114.973234 & -114.972806 & -114.971945 \\
\hline 4.5 & 1.8 & -114.965637 & -114.973349 & -114.972342 & -114.971902 & -114.971082 \\
\hline 4.5 & 2.0 & -114.951402 & -114.959809 & -114.958687 & -114.958209 & -114.957536 \\
\hline 4.5 & 2.25 & -114.922272 & -114.931681 & -114.930384 & -114.929860 & -114.929393 \\
\hline 4.5 & 2.5 & -114.890173 & -114.900784 & -114.899257 & -114.898684 & -114.898413 \\
\hline 4.5 & 2.75 & -114.859607 & -114.871702 & -114.869857 & -114.869223 & -114.869108 \\
\hline 4.5 & 3.0 & -114.832283 & -114.846271 & -114.843960 & -114.843237 & -114.843260 \\
\hline 4.5 & 3.5 & -114.788880 & -114.808787 & -114.804619 & -114.803494 & -114.804248 \\
\hline 4.5 & 4.0 & -114.759229 & -114.790888 & -114.781775 & -114.779393 & -114.781782 \\
\hline 4.7 & 1.2 & -114.768954 & -114.774948 & -114.774204 & -114.773879 & -114.772864 \\
\hline 4.7 & 1.4 & -114.910932 & -114.917384 & -114.916570 & -114.916210 & -114.915236 \\
\hline 4.7 & 1.6 & -114.960350 & -114.967328 & -114.966432 & 66036 & -114.965101 \\
\hline 4.7 & 1.7325 & -114.967253 & -114.974618 & -114.973660 & -114.973239 & -114.972373 \\
\hline 4.7 & 1.8 & -114.966087 & -114.973660 & -114.972668 & -114.972235 & -114.971411 \\
\hline 4.7 & 2.0 & -114.951547 & -114.959789 & 58686 & -114. & 57536 \\
\hline 4.7 & 2.25 & -114.921989 & 31195 & 29920 & 29405 & 28932 \\
\hline 4.7 & 2.5 & -114.889433 & -114.899798 & -114.898298 & -114.897734 & -114.897463 \\
\hline 4.7 & 2.75 & -114.858410 & -114.870217 & -114.868403 & -114.867778 & -114.867681 \\
\hline 4.7 & 3.0 & 0661 & -114 & -114 & -114.8 & -114.841413 \\
\hline 4.7 & 3.5 & 86601 & -114.806140 & -114.802009 & -114.800893 & -114.801800 \\
\hline 4.7 & 4.0 & -114.756610 & -114.787754 & -114.778730 & -114.776380 & -114.778894 \\
\hline 5.0 & 1.2 & -114.770008 & -114.775890 & -114.775159 & -114.774839 & -114.773831 \\
\hline 5.0 & 1.4 & -114.911796 & -114 & -114. & -114.916971 & -114.916002 \\
\hline 5.0 & 1.6 & -114.960976 & -114.967812 & -114.966933 & -114.966543 & -114.965608 \\
\hline 5.0 & 1.7325 & -114.967695 & -114.974902 & -114.973963 & -114.973549 & -114.972682 \\
\hline 5.0 & 1.8 & -114.966426 & -114.973833 & -114.972861 & -114.972435 & -114.971609 \\
\hline 5.0 & 2.0 & -114.951555 & -114.959600 & -114.958518 & -114. & -114.957375 \\
\hline 5.0 & 2.25 & -114.921532 & -114.930494 & -114.929246 & -114.928740 & -114.928268 \\
\hline 5.0 & 2.5 & -114.888483 & -114.898549 & -114.897080 & -114.896526 & -114.896270 \\
\hline 5.0 & 2.75 & -114.856976 & -114.868424 & -114.866646 & -114.866031 & -114.865983 \\
\hline 5.0 & 3.0 & -114.828790 & -114.842043 & -114.839805 & -114.839098 & -114.839289 \\
\hline 5.0 & 3.5 & -114.784145 & -114.803210 & -114.799105 & -114.797989 & -114.799165 \\
\hline 5.0 & 4.0 & -114.754034 & -114.784564 & -114.775559 & -114.773230 & -114.775991 \\
\hline 5.2 & 1.2 & -114.770436 & -114.776258 & -114.775534 & -114.775218 & -114.774214 \\
\hline 5.2 & 1.4 & -114.912130 & -114.918392 & -114.917601 & -114.917250 & -114.916286 \\
\hline 5.2 & 1.6 & -114.961196 & -114.967957 & -114.967086 & -114.966700 & -114.965768 \\
\hline 5.2 & 1.7325 & -114.967826 & -114.974950 & -114.974020 & -114.973611 & -114.972744 \\
\hline 5.2 & 1.8 & -114.966508 & -114.973827 & -114.972865 & -114.972443 & -114.971618 \\
\hline 5.2 & 2.0 & -114.951479 & -114.959419 & -114.958350 & -114.957893 & -114.957212 \\
\hline 5.2 & 2.25 & -114.921235 & -114.930068 & -114.928833 & -114.928333 & -114.927865 \\
\hline 5.2 & 2.5 & -114.887955 & -114.897860 & -114.896407 & -114.895859 & -114.895618 \\
\hline 5.2 & 2.75 & -114.856226 & -114.867478 & -114.865719 & -114.865109 & -114.865098 \\
\hline 5.2 & 3.0 & -114.827852 & -114.840874 & -114.838656 & -114.837952 & -114.838222 \\
\hline 5.2 & 3.5 & -114.783015 & -114.801794 & -114.797701 & -114.796581 & -114.797936 \\
\hline 5.2 & 4.0 & -114.753002 & -114.783135 & -114.774133 & -114.771812 & -114.774758 \\
\hline 5.5 & 1.2 & -114.770813 & -114.776565 & -114.775849 & -114.775536 & -114.774539 \\
\hline
\end{tabular}


Table S8. (continued)

\begin{tabular}{|c|c|c|c|c|c|c|}
\hline$R_{\mathrm{Be}-\mathrm{H}}$ & $R_{\mathrm{H}-\mathrm{F}}$ & CCSD & $\operatorname{CCSD}(\mathrm{T})$ & $\mathrm{R}-\mathrm{CCSD}(\mathrm{T})$ & CR-CCSD(T) & MRCI(Q) \\
\hline 5.5 & 1.4 & -114.912402 & -114.918587 & -114.917804 & -114.917458 & -114.916500 \\
\hline 5.5 & 1.6 & -114.961342 & -114.968014 & -114.967154 & -114.966773 & -114.965844 \\
\hline 5.5 & 1.7325 & -114.967876 & -114.974902 & -114.973984 & -114.973579 & -114.972716 \\
\hline 5.5 & 1.8 & -114.966506 & -114.973722 & -114.972771 & -114.972354 & -114.971532 \\
\hline 5.5 & 2.0 & -114.951306 & -114.959124 & -114.958068 & -114.957617 & -114.956940 \\
\hline 5.5 & 2.25 & -114.920828 & -114.929506 & -114.928288 & -114.927794 & -114.927336 \\
\hline 5.5 & 2.5 & -114.887306 & -114.897017 & -114.895584 & -114.895042 & -114.894824 \\
\hline 5.5 & 2.75 & -114.855351 & -114.866364 & -114.864627 & -114.864022 & -114.864066 \\
\hline 5.5 & 3.0 & -114.826799 & -114.839533 & -114.837339 & -114.836638 & -114.837016 \\
\hline 5.5 & 3.5 & -114.781865 & -114.800249 & -114.796178 & -114.795052 & -114.796645 \\
\hline 5.5 & 4.0 & -114.752141 & -114.781627 & -114.772680 & -114.770375 & -114.773582 \\
\hline 6.0 & 1.2 & -114.771030 & -114.776703 & -114.775996 & -114.775687 & -114.774698 \\
\hline 6.0 & 1.4 & -114.912508 & -114.918604 & -114.917832 & -114.917490 & -114.916537 \\
\hline 6.0 & 1.6 & -114.961322 & -114.967894 & -114.967045 & -114.966669 & -114.965749 \\
\hline 6.0 & 1.7325 & -114.967763 & -114.974678 & -114.973772 & -114.973373 & -114.972517 \\
\hline 6.0 & 1.8 & -114.966342 & -114.973440 & -114.972503 & -114.972092 & -114.971277 \\
\hline 6.0 & 2.0 & -114.950978 & -114.958657 & -114.957616 & -114.957171 & -114.956503 \\
\hline 6.0 & 2.25 & -114.920277 & -114.928779 & -114.927579 & -114.927092 & -114.926650 \\
\hline 6.0 & 2.5 & -114.886530 & -114.896016 & -114.894607 & -114.894071 & -114.893888 \\
\hline 6.0 & 2.75 & -114.854378 & -114.865108 & -114.863399 & -114.862799 & -114.862915 \\
\hline 6.0 & 3.0 & -114.825698 & -114.838077 & -114.835917 & -114.835217 & -114.835729 \\
\hline 6.0 & 3.5 & -114.780853 & -114.798669 & -114.794659 & -114.793526 & -114.795401 \\
\hline 6.0 & 4.0 & -114.751708 & -114.780023 & -114.771322 & -114.769062 & -114.772610 \\
\hline 8.0 & 1.2 & -114.770719 & -114.776283 & -114.775588 & -114.775286 & -114.774315 \\
\hline 8.0 & 1.4 & -114.912091 & -114.918068 & -114.917309 & -114.916974 & -114.916043 \\
\hline 8.0 & 1.6 & -114.960799 & -114.967238 & -114.966404 & -114.966035 & -114.965133 \\
\hline 8.0 & 1.7325 & -114.967164 & -114.973933 & -114.973043 & -114.972651 & -114.971816 \\
\hline 8.0 & 1.8 & -114.965701 & -114.972645 & -114.971724 & -114.971322 & -114.970528 \\
\hline 8.0 & 2.0 & -114.950200 & -114.957696 & -114.956675 & -114.956238 & -114.955595 \\
\hline 8.0 & 2.25 & -114.919308 & -114.927579 & -114.926404 & -114.925927 & -114.925518 \\
\hline 8.0 & 2.5 & -114.885380 & -114.894571 & -114.893192 & -114.892666 & -114.892537 \\
\hline 8.0 & 2.75 & -114.853100 & -114.863449 & -114.861781 & -114.861188 & -114.861397 \\
\hline 8.0 & 3.0 & -114.824401 & -114.836281 & -114.834179 & -114.833482 & -114.834149 \\
\hline 8.0 & 3.5 & -114.780041 & -114.796851 & -114.793036 & -114.791905 & -114.794073 \\
\hline 8.0 & 4.0 & -114.752060 & -114.777575 & -114.769875 & -114.767772 & -114.771775 \\
\hline
\end{tabular}

Table S9. The CCSD, CCSD(T), R-CCSD(T), CR-CCSD(T), and MRCI(Q) energies, in hartree, of the BeFH system, as described by the cc-pVQZ basis set, for the $\mathrm{Be}-\mathrm{F}-\mathrm{H}$ angle $\theta=180^{\circ}$. The Be-F and $\mathrm{H}-\mathrm{F}$ bond distances, $R_{\mathrm{Be}-\mathrm{F}}$ and $R_{\mathrm{H}-\mathrm{F}}$, respectively, are in bohr.

\begin{tabular}{lcccccc}
\hline$R_{\mathrm{Be}-\mathrm{F}}$ & $R_{\mathrm{H}-\mathrm{F}}$ & $\mathrm{CCSD}$ & $\mathrm{CCSD}(\mathrm{T})$ & $\mathrm{R}-\mathrm{CCSD}(\mathrm{T})$ & $\mathrm{CR}-\mathrm{CCSD}(\mathrm{T})$ & $\mathrm{MRCI}(\mathrm{Q})$ \\
\hline 1.8 & 1.2 & -114.415636 & -114.424373 & -114.423531 & -114.423098 & -114.420991 \\
1.8 & 1.4 & -114.559409 & -114.568672 & -114.567755 & -114.567281 & -114.565327 \\
1.8 & 1.6 & -114.611836 & -114.621759 & -114.620732 & -114.620213 & -114.618458 \\
1.8 & 1.7325 & -114.621666 & -114.632226 & -114.631055 & -114.630508 & -114.628928 \\
\hline
\end{tabular}


Table S9. (continued)

\begin{tabular}{|c|c|c|c|c|c|c|}
\hline$R_{\mathrm{Be}-\mathrm{F}}$ & $R_{\mathrm{H}-\mathrm{F}}$ & CCSD & $\operatorname{CCSD}(\mathrm{T})$ & R-CCSD $(\mathrm{T})$ & CR-CCSD(T) & MRCI(Q) \\
\hline 1.8 & 1.8 & -114.622521 & -114.633589 & -114.632265 & -114.631709 & -114.630146 \\
\hline 1.8 & 2.0 & -114.619857 & -114.636926 & -114.632854 & -114.632196 & -114.633552 \\
\hline 1.8 & 2.25 & -114.632426 & -114.673871 & -114.655233 & -114.651861 & -114.657601 \\
\hline 1.8 & 2.5 & -114.654597 & -114.692409 & -114.675652 & -114.673266 & -114.679904 \\
\hline 1.8 & 2.75 & -114.673059 & -114.706285 & -114.691454 & -114.690023 & -114.696658 \\
\hline 1.8 & 3.0 & -114.686905 & -114.718842 & -114.703693 & -114.702297 & -114.708634 \\
\hline 1.8 & 3.5 & -114.704092 & -114.737219 & -114.719476 & -114.717260 & -114.722811 \\
\hline 1.8 & 4.0 & -114.712509 & -114.747056 & -114.727206 & -114.724387 & -114.729376 \\
\hline 1.8 & 5.0 & -114.718205 & -114.752959 & -114.731840 & -114.728865 & -114.733468 \\
\hline 1.8 & 6.0 & -114.719267 & -114.752721 & -114.732112 & -114.729466 & -114.734049 \\
\hline 1.8 & 8.0 & -114.719464 & -114.750295 & -114.731418 & -114.729401 & -114.734031 \\
\hline 1.9 & 1.2 & -114.518482 & -114.527283 & -114.526406 & -114.525971 & -114.523759 \\
\hline 1.9 & 1.4 & -114.662255 & -114.671590 & -114.670638 & -114.670160 & -114.668114 \\
\hline 1.9 & 1.6 & -114.714677 & -114.724687 & -114.723624 & -114.723099 & -114.721283 \\
\hline 1.9 & 1.7325 & -114.724552 & -114.735212 & -114.734007 & -114.733452 & -114.731863 \\
\hline 1.9 & 1.8 & -114.725450 & -114.736617 & -114.735268 & -114.734702 & -114.733220 \\
\hline 1.9 & 2.0 & -114.722608 & -114.738795 & -114.735290 & -114.734641 & -114.735183 \\
\hline 1.9 & 2.25 & -114.732872 & -114.770525 & -114.754635 & -114.751639 & -114.757105 \\
\hline 1.9 & 2.5 & -114.753868 & -114.790832 & -114.774830 & -114.772307 & -114.778670 \\
\hline 1.9 & 2.75 & -114.771818 & -114.804285 & -114.790138 & -114.788635 & -114.795043 \\
\hline 1.9 & 3.0 & -114.785319 & -114.815912 & -114.801843 & -114.800577 & -114.806770 \\
\hline 1.9 & 3.5 & -114.802045 & -114.832753 & -114.816845 & -114.815025 & -114.820620 \\
\hline 1.9 & 4.0 & -114.810204 & -114.841885 & -114.824208 & -114.821844 & -114.826992 \\
\hline 1.9 & 5.0 & -114.815699 & -114.847748 & -114.828694 & -114.826093 & -114.830926 \\
\hline 1.9 & 6.0 & -114.816709 & -114.847908 & -114.829018 & -114.826652 & -114.831472 \\
\hline 1.9 & 8.0 & -114.816884 & -114.846118 & -114.828440 & -114.826595 & -114.831445 \\
\hline 2.0 & 1.2 & -114.594063 & -114.602920 & -114.602011 & -114.601572 & -114.599247 \\
\hline 2.0 & 1.4 & -114.737845 & -114.747243 & -114.746258 & -114.745776 & -114.743642 \\
\hline 2.0 & 1.6 & -114.790267 & -114.800351 & -114.799254 & -114.798725 & -114.796840 \\
\hline 2.0 & 1.7325 & -114.800159 & -114.810896 & -114.809663 & -114.809102 & -114.807480 \\
\hline 2.0 & 1.8 & -114.801062 & -114.812294 & -114.810929 & -114.810355 & -114.808905 \\
\hline 2.0 & 2.0 & -114.797804 & -114.813279 & -114.810211 & -114.809569 & -114.809385 \\
\hline 2.0 & 2.25 & -114.805465 & -114.839966 & -114.826312 & -114.823668 & -114.828843 \\
\hline 2.0 & 2.5 & -114.825009 & -114.861460 & -114.846008 & -114.843349 & -114.849497 \\
\hline 2.0 & 2.75 & -114.842348 & -114.874529 & -114.860776 & -114.859144 & -114.865389 \\
\hline 2.0 & 3.0 & -114.855467 & -114.885305 & -114.871930 & -114.870709 & -114.876804 \\
\hline 2.0 & 3.5 & -114.871717 & -114.900736 & -114.886137 & -114.884620 & -114.890266 \\
\hline 2.0 & 4.0 & -114.879622 & -114.909166 & -114.893114 & -114.891128 & -114.896425 \\
\hline 2.0 & 5.0 & -114.884927 & -114.914831 & -114.897412 & -114.895144 & -114.900193 \\
\hline 2.0 & 6.0 & -114.885891 & -114.915249 & -114.897766 & -114.895656 & -114.900701 \\
\hline 2.0 & 8.0 & -114.886046 & -114.913951 & -114.897282 & -114.895597 & -114.900664 \\
\hline 2.2 & 1.2 & -114.689754 & -114.698699 & -114.697728 & -114.697286 & -114.694745 \\
\hline 2.2 & 1.4 & -114.833542 & -114.843040 & -114.841991 & -114.841505 & -114.839184 \\
\hline 2.2 & 1.6 & -114.885933 & -114.896127 & -114.894970 & -114.894433 & -114.892406 \\
\hline 2.2 & 1.7325 & -114.895773 & -114.906609 & -114.905330 & -114.904758 & -114.903034 \\
\hline 2.2 & 1.8 & -114.896603 & -114.907897 & -114.906511 & -114.905922 & -114.904428 \\
\hline 2.2 & 2.0 & -114.892151 & -114.906560 & -114.904100 & -114.903458 & -114.902320 \\
\hline 2.2 & 2.25 & -114.894069 & -114.923123 & -114.913164 & -114.911199 & -114.915669 \\
\hline 2.2 & 2.5 & -114.909814 & -114.945538 & -114.931022 & -114.928183 & -114.933970 \\
\hline
\end{tabular}


Table S9. (continued)

\begin{tabular}{|c|c|c|c|c|c|c|}
\hline$R_{\mathrm{Be}-\mathrm{F}}$ & $R_{\mathrm{H}-\mathrm{F}}$ & CCSD & $\operatorname{CCSD}(\mathrm{T})$ & $\mathrm{R}-\mathrm{CCSD}(\mathrm{T})$ & CR-CCSD $(\mathrm{T})$ & MRCI(Q) \\
\hline 2.2 & 2.75 & -114.925619 & -114.958159 & -114.944646 & -114.942650 & -114.948647 \\
\hline 2.2 & 3.0 & -114.937873 & -114.967460 & -114.954697 & -114.953356 & -114.959292 \\
\hline 2.2 & 3.5 & -114.953146 & -114.980338 & -114.967308 & -114.966160 & -114.971856 \\
\hline 2.2 & 4.0 & -114.960574 & -114.987394 & -114.973481 & -114.972059 & -114.977562 \\
\hline 2.2 & 5.0 & -114.965536 & -114.992400 & -114.977327 & -114.975612 & -114.980999 \\
\hline 2.2 & 6.0 & -114.966419 & -114.993072 & -114.977685 & -114.976020 & -114.981433 \\
\hline 2.2 & 8.0 & -114.966540 & -114.992451 & -114.977334 & -114.975937 & -114.981375 \\
\hline 2.4 & 1.2 & -114.740191 & -114.749192 & -114.748166 & -114.747722 & -114.746996 \\
\hline 2.4 & 1.4 & -114.883938 & -114.893498 & -114.892395 & -114.891905 & -114.891106 \\
\hline 2.4 & 1.6 & -114.936223 & -114.946481 & -114.945272 & -114.944731 & -114.942577 \\
\hline 2.4 & 1.7325 & -114.945920 & -114.956798 & -114.955481 & -114.954902 & -114.953058 \\
\hline 2.4 & 1.8 & -114.946608 & -114.957902 & -114.956499 & -114.955902 & -114.954289 \\
\hline 2.4 & 2.0 & -114.940805 & -114.954471 & -114.952378 & -114.951727 & -114.950682 \\
\hline 2.4 & 2.25 & -114.936818 & -114.961145 & -114.954139 & -114.952756 & -114.955847 \\
\hline 2.4 & 2.5 & -114.947442 & -114.981938 & 86678 & 65909 & 71294 \\
\hline 2.4 & 2.75 & -114.961165 & -114.994666 & -114.981069 & -114.978684 & -114.984477 \\
\hline 2.4 & 3.0 & -114.972391 & -115.002870 & -114.990053 & -114.988396 & -114.994215 \\
\hline 2.4 & 3.5 & 6644 & -115 . & 01097 & 0049 & 5770 \\
\hline 2.4 & 4.0 & 93626 & -115 . & 6444 & 5355 & 1002 \\
\hline 2.4 & 5.0 & -114.998290 & -115.023389 & -115.009760 & -115.008456 & -115.014102 \\
\hline 2.4 & 6.0 & -114.999106 & -115.024088 & -115.010067 & -115.008753 & -115.014461 \\
\hline 2.4 & 8.0 & -114 & -115 . & -115 . & -115 . & -115.014378 \\
\hline 2.5 & 1.2 & 55434 & -114. & 3402 & -114 & 62172 \\
\hline 2.5 & 1.4 & -114.899135 & -114.908714 & -114.907587 & -114.907097 & -114.906190 \\
\hline 2.5 & 1.6 & -114.951342 & -114.961616 & -114.960385 & -114.959842 & -114.959111 \\
\hline 2.5 & 1.7325 & 0941 & -114 & -114 & -114 & 67990 \\
\hline 2.5 & 1.8 & -114.961541 & -114.972819 & -114.971408 & -114.970808 & -114.968716 \\
\hline 2.5 & 2.0 & -114.955067 & -114.968443 & -114.966473 & -114.965816 & -114.965052 \\
\hline 2.5 & 2.25 & -114.948255 & -114.970541 & -114.964718 & -114.963552 & -114.965803 \\
\hline 2.5 & 2.5 & 5822 & -114 & -114 & -114. & -114.979424 \\
\hline 2.5 & 2.75 & -114.968214 & -115.002194 & -114.988572 & -114.986032 & -114.991729 \\
\hline 2.5 & 3.0 & -114.978835 & -115.010031 & -114.997037 & -114.995185 & -115.000955 \\
\hline 2.5 & 3.5 & -114.992544 & -115.019664 & -115.007317 & -115.006234 & -115.011964 \\
\hline 2.5 & 4.0 & -114.999309 & -115.024752 & -115.012245 & -115.011245 & -115.016951 \\
\hline 2.5 & 5.0 & -115.003836 & -115.028435 & -115.015272 & -115 . & -115.019880 \\
\hline 2.5 & 6.0 & -115.004624 & -115.029091 & -115.015534 & -115.014362 & -115.020202 \\
\hline 2.5 & 8.0 & -115.004705 & -115.028986 & -115.015276 & -115.014215 & -115.020106 \\
\hline 2.5719 & 1.2 & -114.763686 & -114.772709 & -114.771645 & -114.771201 & -114.770376 \\
\hline 2.5719 & 1.4 & -114.907346 & -114.916933 & -114.915790 & -114.915299 & -114.914321 \\
\hline 2.5719 & 1.6 & -114.959484 & -114.969764 & -114.968519 & -114.967976 & -114.967134 \\
\hline 2.5719 & 1.7325 & -114.969002 & -114.979879 & -114.978534 & -114.977953 & -114.976084 \\
\hline 2.5719 & 1.8 & -114.969534 & -114.980795 & -114.979379 & -114.978778 & -114.976761 \\
\hline 2.5719 & 2.0 & -114.962589 & -114.975782 & -114.973881 & -114.973220 & -114.972582 \\
\hline 2.5719 & 2.25 & -114.953844 & -114.974824 & -114.969721 & -114.968676 & -114.970254 \\
\hline 2.5719 & 2.5 & -114.959035 & -114.991522 & -114.979879 & -114.977415 & -114.982373 \\
\hline 2.5719 & 2.75 & -114.970316 & -115.004569 & -114.990978 & -114.988357 & -114.993982 \\
\hline 2.5719 & 3.0 & -114.980450 & -115.012228 & -114.999074 & -114.997076 & -115.002813 \\
\hline 2.5719 & 3.5 & -114.993747 & -115.021206 & -115.008815 & -115.007677 & -115.013411 \\
\hline 2.5719 & 4.0 & -115.000356 & -115.025839 & -115.013440 & -115.012474 & -115.018215 \\
\hline
\end{tabular}


Table S9. (continued)

\begin{tabular}{|c|c|c|c|c|c|c|}
\hline$R_{\mathrm{Be}-\mathrm{F}}$ & $R_{\mathrm{H}-\mathrm{F}}$ & CCSD & $\operatorname{CCSD}(\mathrm{T})$ & $\mathrm{R}-\mathrm{CCSD}(\mathrm{T})$ & CR-CCSD $(\mathrm{T})$ & MRCI(Q) \\
\hline 2.5719 & 5.0 & -115.004789 & -115.029167 & -115.016249 & -115.015190 & -115.021021 \\
\hline 2.5719 & 6.0 & -115.005559 & -115.029770 & -115.016472 & -115.015387 & -115.021316 \\
\hline 2.5719 & 8.0 & -115.005634 & -115.029726 & -115.016220 & -115.015222 & -115.021210 \\
\hline 2.6 & 1.2 & -114.766422 & -114.775447 & -114.774377 & -114.773933 & -114.773092 \\
\hline 2.6 & 1.4 & -114.910064 & -114.919653 & -114.918504 & -114.918014 & -114.917177 \\
\hline 2.6 & 1.6 & -114.962173 & -114.972454 & -114.971203 & -114.970660 & -114.969779 \\
\hline 2.6 & 1.7325 & -114.971657 & -114.982531 & -114.981182 & -114.980601 & -114.979936 \\
\hline 2.6 & 1.8 & -114.972162 & -114.983415 & -114.981997 & -114.981395 & -114.979406 \\
\hline 2.6 & 2.0 & -114.965035 & -114.978161 & -114.976283 & -114.975621 & -114.975007 \\
\hline 2.6 & 2.25 & -114.955561 & -114.976068 & -114.971218 & -114.970213 & -114.971522 \\
\hline 2.6 & 2.5 & -114.959790 & -114.991851 & -114.980522 & -114.978126 & -114.982999 \\
\hline 2.6 & 2.75 & -114.970597 & -115.004931 & -114.991368 & -114.988724 & -114.994321 \\
\hline 2.6 & 3.0 & -114.980527 & -115.012540 & -114.999320 & -114.997266 & -115.002990 \\
\hline 2.6 & 3.5 & -114.993656 & -115.021278 & -115.008853 & -115.007687 & -115.013423 \\
\hline 2.6 & 4.0 & -115.000204 & -115.025736 & -115.013359 & -115.012400 & -115.018155 \\
\hline 2.6 & 5.0 & -115.004601 & -115.028922 & -115.016081 & -115.015052 & -115.020912 \\
\hline 2.6 & 6.0 & -115.005365 & -115.029501 & -115.016288 & -115.015233 & -115.021196 \\
\hline 2.6 & 8.0 & -115.005437 & -115.029475 & -115.016037 & -115.015061 & 21086 \\
\hline 2.7 & 1.2 & -114.774393 & -114.783417 & -114.782330 & 781887 & 780992 \\
\hline 2.7 & 1.4 & -114.917962 & -114.927552 & -114.926386 & -114.925895 & -114.924992 \\
\hline 2.7 & 1.6 & -114.969954 & -114.980233 & -114.978965 & -114.978422 & -114.976706 \\
\hline 2.7 & 1.7325 & -114. & -114.990164 & -114 & -114.988221 & 87348 \\
\hline 2.7 & 1.8 & -114 & -114.990930 & -114 & -114 & 88181 \\
\hline 2.7 & 2.0 & -114.971953 & -114.984858 & -114.983051 & -114.982386 & -114.981752 \\
\hline 2.7 & 2.25 & -114.960019 & -114.979015 & -114.974941 & -114.974044 & -114.974385 \\
\hline 2.7 & 2.5 & -114.960703 & -114.991053 & -114.980924 & -114.978802 & 83324 \\
\hline 2.7 & 2.75 & -114.969630 & -115.004094 & -114.990731 & -114.988050 & -114.993535 \\
\hline 2.7 & 3.0 & -114.978755 & -115.011622 & -114.998161 & -114.995920 & -115.001598 \\
\hline 2.7 & 3.5 & -114.991255 & -115.019588 & -115.006966 & -115.005680 & -115.011419 \\
\hline 2.7 & 4.0 & -114.997583 & -115.023435 & -115.011052 & -115.010090 & -115.015886 \\
\hline 2.7 & 5.0 & -115.001857 & -115.026100 & -115.013454 & -115.012515 & -115.018467 \\
\hline 2.7 & 6.0 & -115.002600 & -115.026574 & -115.013595 & -115.012636 & -115.018713 \\
\hline 2.7 & 8.0 & -115.002664 & -115.026590 & -115.013340 & -115.012435 & -115.018589 \\
\hline 2.9 & 1.2 & -114.784696 & -114.793695 & -114.792584 & -114.792142 & -114.791138 \\
\hline 2.9 & 1.4 & -114.928072 & -114.937638 & -114.936447 & -114.935957 & -114.934933 \\
\hline 2.9 & 1.6 & -114.979770 & -114.990016 & -114.988724 & -114.988181 & -114.986526 \\
\hline 2.9 & 1.7325 & -114.988822 & -114.999619 & -114.998241 & -114.997660 & -114.995990 \\
\hline 2.9 & 1.8 & -114.989010 & -115.000141 & -114.998707 & -114.998104 & -114.996383 \\
\hline 2.9 & 2.0 & -114.980069 & -114.992599 & -114.990886 & -114.990218 & -114.989399 \\
\hline 2.9 & 2.25 & -114.963887 & -114.980602 & -114.977579 & -114.976787 & -114.975798 \\
\hline 2.9 & 2.5 & -114.957302 & -114.983622 & -114.976044 & -114.974498 & -114.977989 \\
\hline 2.9 & 2.75 & -114.961458 & -114.995171 & -114.982805 & -114.980290 & -114.985483 \\
\hline 2.9 & 3.0 & -114.968501 & -115.002877 & -114.989081 & -114.986570 & -114.992142 \\
\hline 2.9 & 3.5 & -114.979526 & -115.009789 & -114.996493 & -114.994887 & -115.000619 \\
\hline 2.9 & 4.0 & -114.985398 & -115.012499 & -114.999750 & -114.998670 & -115.004527 \\
\hline 2.9 & 5.0 & -114.989443 & -115.014069 & -115.001479 & -115.000631 & -115.006744 \\
\hline 2.9 & 6.0 & -114.990156 & -115.014260 & -115.001460 & -115.000629 & -115.006914 \\
\hline 2.9 & 8.0 & -114.990208 & -115.014262 & -115.001166 & -115.000367 & -115.006760 \\
\hline 3.1 & 1.2 & -114.791050 & -114.799989 & -114.798869 & -114.798430 & -114.797321 \\
\hline
\end{tabular}


Table S9. (continued)

\begin{tabular}{|c|c|c|c|c|c|c|}
\hline$R_{\mathrm{Be}-\mathrm{F}}$ & $R_{\mathrm{H}-\mathrm{F}}$ & CCSD & $\operatorname{CCSD}(\mathrm{T})$ & R-CCSD $(\mathrm{T})$ & CR-CCSD(T) & $\operatorname{MRCI}(\mathrm{Q})$ \\
\hline 3.1 & 1.4 & -114.934160 & -114.943667 & -114.942465 & -114.941978 & -114.940851 \\
\hline 3.1 & 1.6 & -114.985501 & -114.995677 & -114.994374 & -114.993834 & -114.992235 \\
\hline 3.1 & 1.7325 & -114.994222 & -115.004925 & -115.003540 & -115.002961 & -115.001391 \\
\hline 3.1 & 1.8 & -114.994189 & -115.005200 & -115.003765 & -115.003166 & -115.001582 \\
\hline 3.1 & 2.0 & -114.984177 & -114.996386 & -114.994732 & -114.994067 & -114.992183 \\
\hline 3.1 & 2.25 & -114.964621 & -114.979817 & -114.977377 & -114.976617 & -114.976544 \\
\hline 3.1 & 2.5 & -114.951292 & -114.973623 & -114.968256 & -114.967135 & -114.969079 \\
\hline 3.1 & 2.75 & -114.949417 & -114.980738 & -114.970245 & -114.968178 & -114.972886 \\
\hline 3.1 & 3.0 & -114.953486 & -114.988527 & -114.974923 & -114.972386 & -114.977812 \\
\hline 3.1 & 3.5 & -114.962567 & -114.995207 & -114.980994 & -114.979038 & -114.984747 \\
\hline 3.1 & 4.0 & -114.967938 & -114.996996 & -114.983475 & -114.982164 & -114.988051 \\
\hline 3.1 & 5.0 & -114.971782 & -114.997436 & -114.984511 & -114.983650 & -114.989889 \\
\hline 3.1 & 6.0 & -114.972482 & -114.997252 & -114.984298 & -114.983518 & -114.989981 \\
\hline 3.1 & 8.0 & -114.972532 & -114.997125 & -114.983927 & -114.983187 & -114.989793 \\
\hline 3.3 & 1.2 & -114.795811 & -114.804657 & -114.803541 & -114.803105 & -114.801274 \\
\hline 3.3 & 1.4 & -114.938598 & -114.948010 & -114.946810 & -114.946327 & -114.944648 \\
\hline 3.3 & 1.6 & -114.989543 & -114.999613 & -114.998313 & -114.997776 & -114.996223 \\
\hline 3.3 & 1.7325 & -114.997921 & -115.008497 & -115.007117 & -115.006543 & -115.005051 \\
\hline 3.3 & 1.8 & -114.997670 & -115.008534 & -115.007108 & -115.006514 & -115.005036 \\
\hline 3.3 & 2.0 & -114.986701 & -114.998620 & -114.997008 & -114.996350 & -114.994759 \\
\hline 3.3 & 2.25 & -114.964519 & -114.978685 & -114.976562 & -114.975816 & -114.976352 \\
\hline 3.3 & 2.5 & -114.945610 & -114.964765 & -114.960911 & -114.959997 & -114.960367 \\
\hline 3.3 & 2.75 & -114.937071 & -114.964708 & -114.956608 & -114.955076 & -114.958917 \\
\hline 3.3 & 3.0 & -114.937022 & -114.971233 & -114.958699 & -114.956428 & -114.961611 \\
\hline 3.3 & 3.5 & -114.943371 & -114.978466 & -114.963312 & -114.961075 & -114.966745 \\
\hline 3.3 & 4.0 & -114.948145 & -114.979727 & -114.965104 & -114.963505 & -114.969392 \\
\hline 3.3 & 5.0 & -114.951814 & -114.979076 & -114.965464 & -114.964505 & -114.970836 \\
\hline 3.3 & 6.0 & -114.952521 & -114.978432 & -114.965028 & -114.964235 & -114.970847 \\
\hline 3.3 & 8.0 & -114.952579 & -114.978072 & -114.964547 & -114.963826 & -114.970622 \\
\hline 3.5 & 1.2 & -114.799995 & -114.808717 & -114.807615 & -114 & -114.805920 \\
\hline 3.5 & 1.4 & -114.942431 & -114.951717 & -114.950529 & -114.950051 & -114.949492 \\
\hline 3.5 & 1.6 & -114.992966 & -115.002898 & -115.001610 & -115.001080 & -115.000597 \\
\hline 3.5 & 1.7325 & -115.001005 & -115.011425 & -115.010059 & -115.009492 & -115.009040 \\
\hline 3.5 & 1.8 & -115.000548 & -115.011241 & -115.009830 & -115.009243 & -115.008796 \\
\hline 3.5 & 2.0 & -114.988737 & -115.000381 & -114.998806 & -114.998158 & -114.996765 \\
\hline 3.5 & 2.25 & -114.964524 & -114.977959 & -114.976014 & -114.975282 & -114.975385 \\
\hline 3.5 & 2.5 & -114.941366 & -114.958303 & -114.955334 & -114.954495 & -114.954806 \\
\hline 3.5 & 2.75 & -114.926513 & -114.950198 & -114.944323 & -114.943182 & -114.945635 \\
\hline 3.5 & 3.0 & -114.921285 & -114.952948 & -114.942393 & -114.940589 & -114.945310 \\
\hline 3.5 & 3.5 & -114.923688 & -114.960803 & -114.945003 & -114.942653 & -114.948268 \\
\hline 3.5 & 4.0 & -114.927669 & -114.962152 & -114.946206 & -114.944327 & -114.950187 \\
\hline 3.5 & 5.0 & -114.931169 & -114.960581 & -114.945948 & -114.944830 & -114.951214 \\
\hline 3.5 & 6.0 & -114.931907 & -114.959406 & -114.945272 & -114.944412 & -114.951140 \\
\hline 3.5 & 8.0 & -114.931988 & -114.958711 & -114.944652 & -114.943917 & -114.950876 \\
\hline 3.7 & 1.2 & -114.803919 & -114.812495 & -114.811412 & -114.810987 & -114.809670 \\
\hline 3.7 & 1.4 & -114.946004 & -114.955139 & -114.953971 & -114.953498 & -114.952830 \\
\hline 3.7 & 1.6 & -114.996140 & -115.005911 & -115.004642 & -115.004119 & -115.003536 \\
\hline 3.7 & 1.7325 & -115.003860 & -115.014105 & -115.012759 & -115.012199 & -115.011668 \\
\hline 3.7 & 1.8 & -115.003214 & -115.013719 & -115.012330 & -115.011752 & -115.011240 \\
\hline
\end{tabular}


Table S9. (continued)

\begin{tabular}{|c|c|c|c|c|c|c|}
\hline$R_{\mathrm{Be}-\mathrm{F}}$ & $R_{\mathrm{H}-\mathrm{F}}$ & CCSD & $\operatorname{CCSD}(\mathrm{T})$ & R-CCSD $(\mathrm{T})$ & CR-CCSD $(\mathrm{T})$ & $\operatorname{MRCI}(\mathrm{Q})$ \\
\hline 3.7 & 2.0 & -114.990677 & -115.002062 & -115.000520 & -114.999884 & -114.999375 \\
\hline 3.7 & 2.25 & -114.964901 & -114.977788 & -114.975953 & -114.975238 & -114.974382 \\
\hline 3.7 & 2.5 & -114.938660 & -114.954119 & -114.951633 & -114.950826 & -114.952161 \\
\hline 3.7 & 2.75 & -114.918602 & -114.938993 & -114.934710 & -114.933757 & -114.934711 \\
\hline 3.7 & 3.0 & -114.907747 & -114.935706 & -114.927563 & -114.926213 & -114.930041 \\
\hline 3.7 & 3.5 & -114.904631 & -114.942704 & -114.926957 & -114.924720 & -114.930250 \\
\hline 3.7 & 4.0 & -114.907448 & -114.944921 & -114.927616 & -114.925537 & -114.931349 \\
\hline 3.7 & 5.0 & -114.910748 & -114.942816 & -114.926843 & -114.925533 & -114.931928 \\
\hline 3.7 & 6.0 & -114.911546 & -114.941065 & -114.925923 & -114.924956 & -114.931764 \\
\hline 3.7 & 8.0 & -114.911670 & -114.939937 & -114.925142 & -114.924365 & -114.931458 \\
\hline 3.9 & 1.2 & -114.807596 & -114.816012 & -114.814953 & -114.814534 & -114.812412 \\
\hline 3.9 & 1.4 & -114.949353 & -114.958324 & -114.957178 & -114.956713 & -114.954362 \\
\hline 3.9 & 1.6 & -114.999120 & -115.008718 & -115.007472 & -115.006956 & -115.004383 \\
\hline 3.9 & 1.7325 & -115.006552 & -115.016612 & -115.015289 & -115.014738 & -115.014109 \\
\hline 3.9 & 1.8 & -115.005739 & -115.016050 & -115.014686 & -115.014117 & -115.013519 \\
\hline 3.9 & 2.0 & -114.992592 & -115.003731 & -115.002221 & -115.001597 & -115.001057 \\
\hline 3.9 & 2.25 & -114.965617 & -114.978076 & -114.976314 & -114.975615 & -114.974588 \\
\hline 3.9 & 2.5 & -114.937187 & -114.951656 & -114.949433 & -114.948649 & -114.947858 \\
\hline 3.9 & 2.75 & -114.913257 & -114.931297 & -114.927970 & -114.927085 & -114.927979 \\
\hline 3.9 & 3.0 & -114.897206 & -114.921378 & -114.915378 & -114.914305 & -114.916692 \\
\hline 3.9 & 3.5 & -114.887030 & -114.924459 & -114.909789 & -114.907868 & -114.913240 \\
\hline 3.9 & 4.0 & -114.888051 & -114.928177 & -114.909775 & -114.907638 & -114.913383 \\
\hline 3.9 & 5.0 & -114.891041 & -114.926215 & -114.908615 & -114.907113 & -114.913474 \\
\hline 3.9 & 6.0 & -114.891928 & -114.923897 & -114.907467 & -114.906369 & -114.913214 \\
\hline 3.9 & 8.0 & -114.892122 & -114.922246 & -114.906510 & -114.905670 & -114.912865 \\
\hline 4.1 & 1.2 & -114.810949 & -114.819204 & -114.818167 & -114.817756 & -114.815600 \\
\hline 4.1 & 1.4 & -114.952416 & -114.961220 & -114.960097 & -114.959640 & -114.957255 \\
\hline 4.1 & 1.6 & -115.001853 & -115.011276 & -115.010053 & -115.009546 & -115.006937 \\
\hline 4.1 & 1.7325 & -115.009033 & -115.018908 & -115.017610 & -115.017068 & -115.015770 \\
\hline 4.1 & 1.8 & -115.008079 & -115.018197 & -115.016857 & -115.016298 & -115.015028 \\
\hline 4.1 & 2.0 & -114.994428 & -115.005338 & -115.003858 & -115.003246 & -115.002076 \\
\hline 4.1 & 2.25 & -114.966538 & -114.978650 & -114.976941 & -114.976259 & -114.975207 \\
\hline 4.1 & 2.5 & -114.936565 & -114.950346 & -114.948274 & -114.947514 & -114.946558 \\
\hline 4.1 & 2.75 & -114.909930 & -114.926403 & -114.923600 & -114.922747 & -114.923437 \\
\hline 4.1 & 3.0 & -114.889724 & -114.910831 & -114.906319 & -114.905354 & -114.906308 \\
\hline 4.1 & 3.5 & -114.871593 & -114.906681 & -114.894090 & -114.892561 & -114.897567 \\
\hline 4.1 & 4.0 & -114.869871 & -114.911775 & -114.892930 & -114.890907 & -114.896564 \\
\hline 4.1 & 5.0 & -114.872300 & -114.910933 & -114.891491 & -114.889832 & -114.896112 \\
\hline 4.1 & 6.0 & -114.873301 & -114.908154 & -114.890151 & -114.888915 & -114.895751 \\
\hline 4.1 & 8.0 & -114.873596 & -114.905908 & -114.889012 & -114.888095 & -114.895357 \\
\hline 4.5 & 1.2 & -114.816430 & -114.824384 & -114.823388 & -114.822992 & -114.820684 \\
\hline 4.5 & 1.4 & -114.957433 & -114.965925 & -114.964844 & -114.964402 & -114.961872 \\
\hline 4.5 & 1.6 & -115.006347 & -115.015442 & -115.014262 & -115.013773 & -115.011027 \\
\hline 4.5 & 1.7325 & -115.013137 & -115.022669 & -115.021415 & -115.020893 & -115.019504 \\
\hline 4.5 & 1.8 & -115.011967 & -115.021733 & -115.020438 & -115.019900 & -115.018542 \\
\hline 4.5 & 2.0 & -114.997587 & -115.008097 & -115.006668 & -115.006080 & -115.004832 \\
\hline 4.5 & 2.25 & -114.968473 & -114.980052 & -114.978417 & -114.977765 & -114.976670 \\
\hline 4.5 & 2.5 & -114.936636 & -114.949547 & -114.947628 & -114.946908 & -114.945929 \\
\hline 4.5 & 2.75 & -114.906937 & -114.921677 & -114.919318 & -114.918514 & -114.917563 \\
\hline
\end{tabular}


Table S9. (continued)

\begin{tabular}{|c|c|c|c|c|c|c|}
\hline$R_{\mathrm{Be}-\mathrm{F}}$ & $R_{\mathrm{H}-\mathrm{F}}$ & CCSD & $\operatorname{CCSD}(\mathrm{T})$ & $\mathrm{R}-\mathrm{CCSD}(\mathrm{T})$ & CR-CCSD $(\mathrm{T})$ & $\operatorname{MRCI}(\mathrm{Q})$ \\
\hline 4.5 & 3.0 & -114.881735 & -114.899255 & -114.896097 & -114.895188 & -114.894238 \\
\hline 4.5 & 3.5 & -114.849300 & -114.877392 & -114.869649 & -114.868519 & -114.871219 \\
\hline 4.5 & 4.0 & -114.838620 & -114.879636 & -114.863124 & -114.861634 & -114.866839 \\
\hline 4.5 & 5.0 & -114.838102 & -114.883967 & -114.860856 & -114.859099 & -114.865052 \\
\hline 4.5 & 6.0 & -114.839308 & -114.881210 & -114.859284 & -114.857825 & -114.864466 \\
\hline 4.5 & 8.0 & -114.839919 & -114.877741 & -114.857795 & -114.856709 & -114.863982 \\
\hline 4.7 & 1.2 & -114.818520 & -114.826344 & -114.825365 & -114.824974 & -114.822666 \\
\hline 4.7 & 1.4 & -114.959346 & -114.967702 & -114.966638 & -114.966205 & -114.963675 \\
\hline 4.7 & 1.6 & -115.008065 & -115.017018 & -115.015856 & -115.015375 & -115.012633 \\
\hline 4.7 & 1.7325 & -115.014712 & -115.024095 & -115.022860 & -115.022347 & -115.020930 \\
\hline 4.7 & 1.8 & -115.013464 & -115.023077 & -115.021802 & -115.021272 & -115.019887 \\
\hline 4.7 & 2.0 & -114.998829 & -115.009170 & -115.007762 & -115.007184 & -115.005912 \\
\hline 4.7 & 2.25 & -114.969314 & -114.980687 & -114.979079 & -114.978440 & -114.977330 \\
\hline 4.7 & 2.5 & -114.936924 & -114.949544 & -114.947669 & -114.946964 & -114.945995 \\
\hline 4.7 & 2.75 & -114.906396 & -114.920647 & -114.918383 & -114.917599 & -114.916697 \\
\hline 4.7 & 3.0 & -114.879881 & -114.896465 & -114.893563 & -114.892674 & -114.891728 \\
\hline 4.7 & 3.5 & -114.842607 & -114.867816 & -114.861662 & -114.860516 & -114.861352 \\
\hline 4.7 & 4.0 & -114.826430 & -114.864873 & -114.850882 & -114.849539 & -114 \\
\hline 4.7 & 5.0 & -114.822754 & -114.871808 & -114.847432 & -114.845732 & 51375 \\
\hline 4.7 & 6.0 & -114.823912 & -114.869939 & -114.845782 & -114.844254 & -114.850661 \\
\hline 4.7 & 8.0 & -114.824723 & -114.866013 & -114.844121 & -114.842950 & -114.850134 \\
\hline 5.0 & 1.2 & -114.820916 & 8572 & -114.827614 & 27232 & -114 \\
\hline 5.0 & 1.4 & -114.961539 & -114.969720 & -114.968678 & -114.968254 & -114.966724 \\
\hline 5.0 & 1.6 & -115.010034 & -115.018803 & -115.017664 & -115.017194 & -115.015701 \\
\hline 5.0 & 1.7325 & -115.016517 & -115.025708 & -115.024497 & -115.023995 & -115.022552 \\
\hline 5.0 & 1.8 & -115.015181 & -115.024597 & -115.023347 & -115.022829 & -115 \\
\hline 5.0 & 2.0 & -115.000260 & -115.010386 & -115.009006 & -115.008442 & 07144 \\
\hline 5.0 & 2.25 & -114.970318 & -114.981438 & -114.979862 & -114.979239 & -114.978114 \\
\hline 5.0 & 2.5 & -114.937372 & -114.949661 & -114.947832 & -114.947148 & -114.946192 \\
\hline 5.0 & 2.75 & -114.906069 & -114.919823 & -114.917641 & -114.916881 & -114.916063 \\
\hline 5.0 & 3.0 & -114.878401 & -114.894122 & -114.891410 & -114.890547 & -114.889820 \\
\hline 5.0 & 3.5 & -114.836758 & -114.859219 & -114.854255 & -114.853053 & -114.852179 \\
\hline 5.0 & 4.0 & -114.813562 & -114.847684 & -114.837161 & -114.835635 & -114.837722 \\
\hline 5.0 & 5.0 & -114.802350 & -114.854791 & -114.830119 & -114.828437 & -114.833142 \\
\hline 5.0 & 6.0 & -114.802760 & -114.855657 & -114.828149 & -114.826514 & -114.832202 \\
\hline 5.0 & 8.0 & -114.803798 & -114.851596 & -114.826134 & -114.824809 & -114.831621 \\
\hline 5.2 & 1.2 & -114.822093 & -114.829656 & -114.828709 & -114.828333 & -114.826026 \\
\hline 5.2 & 1.4 & -114.962615 & -114.970699 & -114.969669 & -114.969251 & -114.966727 \\
\hline 5.2 & 1.6 & -115.010999 & -115.019665 & -115.018539 & -115.018075 & -115.015346 \\
\hline 5.2 & 1.7325 & -115.017399 & -115.026483 & -115.025285 & -115.024791 & -115.023337 \\
\hline 5.2 & 1.8 & -115.016019 & -115.025326 & -115.024089 & -115.023578 & -115.022156 \\
\hline 5.2 & 2.0 & -115.000958 & -115.010965 & -115.009599 & -115.009043 & -115.007736 \\
\hline 5.2 & 2.25 & -114.970814 & -114.981797 & -114.980238 & -114.979625 & -114.978494 \\
\hline 5.2 & 2.5 & -114.937621 & -114.949740 & -114.947934 & -114.947260 & -114.946312 \\
\hline 5.2 & 2.75 & -114.905995 & -114.919515 & -114.917368 & -114.916621 & -114.915845 \\
\hline 5.2 & 3.0 & -114.877882 & -114.893239 & -114.890593 & -114.889743 & -114.889143 \\
\hline 5.2 & 3.5 & -114.834660 & -114.856066 & -114.851436 & -114.850219 & -114.849971 \\
\hline 5.2 & 4.0 & -114.808456 & -114.840488 & -114.831272 & -114.829514 & -114.828765 \\
\hline 5.2 & 5.0 & -114.791158 & -114.845007 & -114.821146 & -114.819126 & -114.816305 \\
\hline
\end{tabular}


Table S9. (continued)

\begin{tabular}{|c|c|c|c|c|c|c|}
\hline$R_{\mathrm{Be}-\mathrm{F}}$ & $R_{\mathrm{H}-\mathrm{F}}$ & CCSD & $\operatorname{CCSD}(\mathrm{T})$ & R-CCSD $(\mathrm{T})$ & CR-CCSD(T) & $\operatorname{MRCI}(\mathrm{Q})$ \\
\hline 5.2 & 6.0 & -114.790092 & -114.848165 & -114.818586 & -114.816725 & -114.821401 \\
\hline 5.2 & 8.0 & -114.791023 & -114.844513 & -114.816087 & -114.814570 & -114.820793 \\
\hline 5.5 & 1.2 & -114.823365 & -114.830813 & -114.829881 & -114.829510 & -114.827206 \\
\hline 5.5 & 1.4 & -114.963775 & -114.971738 & -114.970723 & -114.970311 & -114.967791 \\
\hline 5.5 & 1.6 & -115.012033 & -115.020572 & -115.019462 & -115.019005 & -115.016282 \\
\hline 5.5 & 1.7325 & -115.018341 & -115.027293 & -115.026112 & -115.025625 & -115.024165 \\
\hline 5.5 & 1.8 & -115.016911 & -115.026084 & -115.024863 & -115.024361 & -115.022932 \\
\hline 5.5 & 2.0 & -115.001696 & -115.011557 & -115.010210 & -115.009663 & -115.008350 \\
\hline 5.5 & 2.25 & -114.971336 & -114.982153 & -114.980615 & -114.980014 & -114.978878 \\
\hline 5.5 & 2.5 & -114.937891 & -114.949811 & -114.948030 & -114.947371 & -114.946430 \\
\hline 5.5 & 2.75 & -114.905954 & -114.919217 & -114.917105 & -114.916374 & -114.915640 \\
\hline 5.5 & 3.0 & -114.877441 & -114.892427 & -114.889840 & -114.889007 & -114.888536 \\
\hline 5.5 & 3.5 & -114.832948 & -114.853420 & -114.849011 & -114.847792 & -114.848228 \\
\hline 5.5 & 4.0 & -114.804417 & -114.834658 & -114.826184 & -114.824189 & -114.825543 \\
\hline 5.5 & 5.0 & -114.779864 & -114.836438 & -114.813015 & -114.809489 & -114.810359 \\
\hline 5.5 & 6.0 & -114.774466 & -114.843037 & -114.809646 & -114.806362 & -114.807743 \\
\hline 5.5 & 8.0 & -114.773857 & -114.840277 & -114.805499 & -114.803103 & -114.807176 \\
\hline 6.0 & 1.2 & -114.824568 & -114.831880 & -114.830963 & -114.830600 & -114.828298 \\
\hline 6.0 & 1.4 & -114.964860 & -114.972680 & -114.971682 & -114.971279 & -114.968765 \\
\hline 6.0 & 1.6 & -115.012987 & -115.021376 & -115.020283 & -115.019836 & -115.017122 \\
\hline 6.0 & 1.7325 & -115.019202 & -115.027997 & -115.026834 & -115.026358 & -115.024899 \\
\hline 6.0 & 1.8 & -115.017722 & -115.026734 & -115.025532 & -115.025041 & -115.023614 \\
\hline 6.0 & 2.0 & -115.002352 & -115.012040 & -115.010714 & -115.010179 & -115.008866 \\
\hline 6.0 & 2.25 & -114.971782 & -114.982405 & -114.980892 & -114.980304 & -114.979171 \\
\hline 6.0 & 2.5 & -114.938105 & -114.949801 & -114.948048 & -114.947405 & -114.946474 \\
\hline 6.0 & 2.75 & -114.905905 & -114.918893 & -114.916818 & -114.916105 & -114.915409 \\
\hline 6.0 & 3.0 & -114.877084 & -114.891712 & -114.889176 & -114.888362 & -114.887996 \\
\hline 6.0 & 3.5 & -114.831796 & -114.851522 & -114.847240 & -114.846026 & -114.846982 \\
\hline 6.0 & 4.0 & -114.802188 & -114.831118 & -114.822852 & -114.820729 & -114.823583 \\
\hline 6.0 & 5.0 & -114.774281 & -114.837457 & -114.809679 & -114.803581 & -114.807614 \\
\hline 6.0 & 6.0 & -114.764566 & -114.865084 & -114.812117 & -114.802022 & -114.804846 \\
\hline 6.0 & 8.0 & -114.758342 & -114.886502 & -114.813510 & -114.801993 & -114.804280 \\
\hline 8.0 & 1.2 & -114.825372 & -114.832488 & -114.831593 & -114.831241 & -114.828951 \\
\hline 8.0 & 1.4 & -114.965525 & -114.973138 & -114.972164 & -114.971773 & -114.969273 \\
\hline 8.0 & 1.6 & -115.013502 & -115.021671 & -115.020604 & -115.020171 & -115.017475 \\
\hline 8.0 & 1.7325 & -115.019611 & -115.028177 & -115.027041 & -115.026581 & -115.025150 \\
\hline 8.0 & 1.8 & -115.018077 & -115.026854 & -115.025681 & -115.025206 & -115.023804 \\
\hline 8.0 & 2.0 & -115.002542 & -115.011978 & -115.010682 & -115.010167 & -115.008877 \\
\hline 8.0 & 2.25 & -114.971764 & -114.982108 & -114.980629 & -114.980064 & -114.978952 \\
\hline 8.0 & 2.5 & -114.937882 & -114.949265 & -114.947552 & -114.946935 & -114.946028 \\
\hline 8.0 & 2.75 & -114.905488 & -114.918118 & -114.916089 & -114.915405 & -114.914747 \\
\hline 8.0 & 3.0 & -114.876487 & -114.890694 & -114.888213 & -114.887431 & -114.887141 \\
\hline 8.0 & 3.5 & -114.830933 & -114.849990 & -114.845806 & -114.844618 & -114.845836 \\
\hline 8.0 & 4.0 & -114.801393 & -114.828940 & -114.820964 & -114.818833 & -114.822271 \\
\hline 8.0 & 5.0 & -114.775244 & -114.832575 & -114.806940 & -114.800731 & -114.806203 \\
\hline 8.0 & 6.0 & -114.767661 & -114.871176 & -114.812557 & -114.799841 & -114.803432 \\
\hline 8.0 & 8.0 & -114.764240 & -114.952720 & -114.827955 & -114.804437 & -114.802878 \\
\hline
\end{tabular}

